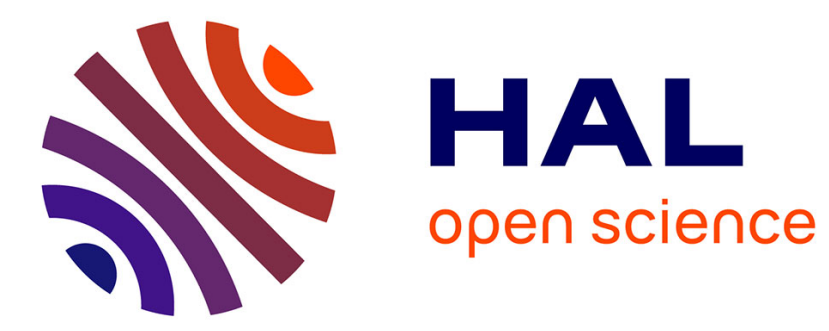

\title{
THE ROLE OF HOST GALAXY KINEMATICS ON NUCLEAR ACTIVITY
}

\author{
Gaelle Dumas
}

\section{To cite this version:}

Gaelle Dumas. THE ROLE OF HOST GALAXY KINEMATICS ON NUCLEAR ACTIVITY. Astrophysics [astro-ph]. Ecole normale supérieure de lyon - ENS LYON; Liverpool John Moores University, 2008. English. NNT: . tel-00350474

\section{HAL Id: tel-00350474 \\ https://theses.hal.science/tel-00350474}

Submitted on 6 Jan 2009

HAL is a multi-disciplinary open access archive for the deposit and dissemination of scientific research documents, whether they are published or not. The documents may come from teaching and research institutions in France or abroad, or from public or private research centers.
L'archive ouverte pluridisciplinaire HAL, est destinée au dépôt et à la diffusion de documents scientifiques de niveau recherche, publiés ou non, émanant des établissements d'enseignement et de recherche français ou étrangers, des laboratoires publics ou privés. 


\section{THÈSE}

en vue d'obtenir le grade de

Docteur de 1'Université de Lyon - École Normale Supérieure de Lyon Spécialité: Astrophysique

Centre de Recherche Astrophysique de Lyon/Observatoire de Lyon École doctorale de Physique et Astrophysique de Lyon

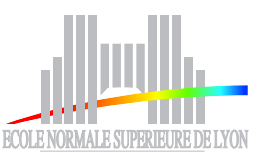

Présentée et soutenue publiquement le 18 septembre 2007 par

\section{GAËLLE DUMAS}

\section{THE ROLE OF HOST GALAXY KINEMATICS ON NUCLEAR}

\section{ACTIVITY}
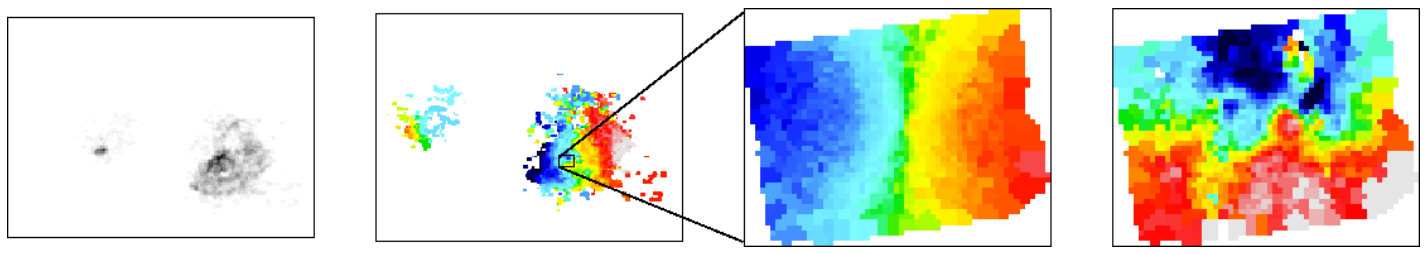

Sous la direction de ERIC EMSELlem ET CAROLE MundeLL

Après avis de la commission d'examen formée de :

$\begin{array}{ll}\text { Niranjan } & \text { Thatte } \\ \text { Phil } & \text { James } \\ \text { Chris } & \text { Collins } \\ \text { Bruno } & \text { Guiderdoni } \\ \text { Eric } & \text { Emsellem } \\ \text { Carole } & \text { Mundell }\end{array}$

Lecturer

Principal Lecturer

Professor

Rapporteur

Directeur de recherche

Examinateur

Astronome

Examinateur

Royal Society Research Fellow

Directeur

Directrice 


\section{Declaration}

The work presented in this thesis was carried out in the Astrophysics Research Institute, Liverpool John Moores University and the Centre de Recherche Astrophysique de Lyon - Observatoire de Lyon, École Normale Supérieure de Lyon, as part of a joint doctorate co-tutorship. Unless otherwise stated, it is the original work of the author.

While registered as a candidate for the degree of Doctor of Philosophy, for which submission is now made, the author has not been registered as a candidate for any other award. This thesis has not been submitted in whole, or in part, for any other degree.

Gaëlle Dumas

Astrophysics Research Institute
Liverpool John Moores University
Twelve Quays House
Egerton Wharf
Birkenhead
CH41 1LD
UK

CRAL - Observatoire de Lyon

9 Avenue Charles André 69561 Saint Genis Laval Cedex France

September 18, 2007

The copyright of this thesis rests with the author. No quotation from it should be published without his prior written consent and information derived from it should be acknowledged @ Gaëlle Dumas (2007). 


\title{
The Role of Host Galaxy Kinematics IN NUCLEAR ACTIVITY.
}

\section{Gaëlle Dumas}

Submitted for the degree of Doctor of Philosophy

ASTROPHYSICS RESEARCH INSTITUTE

CEntre De Recherche AstrophysiQue De LyON -

OBSERVATOIRE DE LYON

September 18, 2007

\begin{abstract}
Most bulge-dominated galaxies host a supermassive black hole and yet ongoing nuclear activity is observed in only $\sim 5 \%$ of nearby galaxies. Re-ignition of dormant black holes is therefore required and a key unanswered question is whether the ignition mechanism is related to the galaxy host properties, in particular the fuel transportation mechanisms. The nuclear activity in distant and luminous quasars is related to galaxy interactions and the high-luminosity active galactic nuclei (AGN) are found in giant elliptical galaxies associated with past mergers of lower mass galaxies. In contrast, the low-luminosity AGN, such as the Seyfert galaxies, are not found preferentially in interacting systems or in barred galaxies and fuelling these low-luminosity AGN remains an unsolved problem. Recent results suggest the presence of identifiable dynamical differences between Seyfert and inactive galaxies in the central kpc regions. Probing the dynamics of Seyfert galaxies requires spectroscopic data and integral-field spectroscopy (IFS) is particularly well-suited to the investigation of complex structures of nearby galaxies. A comprehensive and statistically-significant study of the neutral gas, ionised gas and stellar kinematics of a well-defined distance-limited sample of Seyfert galaxies paired with control inactive galaxies with carefully matched optical properties was then initiated, using the VLA and the SAURON integral field unit (IFU). This project aims to compare the morphological and kinematic properties of active and inactive galaxies on spatial scales from the outer disk (via radio data) to the inner regions (via optical IFS data).
\end{abstract}


This work first focuses on the optical data. The velocity maps reveal regular stellar kinematics in the circumnuclear regions, while the gaseous kinematics presents some disturbances. A first quantitative analysis of the stellar and gaseous velocity fields was conducted. The result of this analysis is an increased incidence of disturbed ionised gas in the circumnuclear regions of the Seyfert galaxies (100 to $10 \mathrm{pc}$ ), which could suggest a link between nuclear host dynamics and fuelling mechanisms of the AGN. Radio and optical data were then combined in order to perform global disk kinematic analysis of the galaxies. Although data reduction techniques differ for optical and radio data, similar methods to analyse the two-dimensional velocity fields can be applied to both datasets. Thus a dedicated kinematic tool using Fourier decomposition associated with the tilted-ring method was developed to analyse the $\mathrm{HI}$ velocity fields and the optical (stars and ionised gas) velocity fields for a wellselected subsample of active and inactive galaxies. This study allows one to obtain the velocity curve at large radii as well as in the inner part $\left(\mathrm{r}<20^{\prime \prime}\right)$. The gaseous kinematic disturbances are then investigated at large and small spatial scales. 


\section{Résumé}

Un trou noir supermassif est présent au centre de la majorité des galaxies incluant une composante sphéroidale, dans l'Univers local. Cependant, seulement 10 à $20 \%$ de ces galaxies montrent des signes d'activité nucléaire. Le redémarrage des processus d'accrétion sur le trou noir (TN) central de ces galaxies est donc nécessaire. Une question importante reste donc de savoir si le réveil du TN et les mécanismes transportant le gaz vers les régions centrales sont liés aux propriétés de la galaxie elle-même. L'émission centrale des quasars lointains est associée aux phénomènes d'interaction entre galaxies et les noyaux actifs proches très lumineux se trouvent dans des galaxies elliptiques géantes résultant très probablement de la fusion de plusieurs galaxies. Les noyaux actifs peu lumineux, comme les galaxies de Seyfert, ne semblent par contre pas se distinguer des galaxies non-actives en terme d'environnement galactique (companions, interaction) ou de structures dynamiques (barres). De récents résultats suggèrent l'existence de différences dynamiques dans le kiloparsec central entre les galaxies de Seyfert et non actives. Des données spectroscopiques sont nécessaires pour étudier la dynamique de ces galaxies et la spectroscopie intégrale de champ (SIC) est bien adaptée à l'étude des régions complexes des galaxies proches. Une étude approfondie et statistique de la cinématique du gaz neutre, du gaz ionisé et des étoiles a été menée sur un échantillon de galaxies de Seyfert associées à des galaxies non actives de contrôle avec des propriétés optiques similaires, en utilisant le VLA (Very Large Array) et le spectrographe intégral de champ SAURON monté au William Herschel Telescope (WHT). Cette étude a pour but de comparer la morphologie et la cinématique des galaxies actives et non actives, des régions les plus externes (grâce aux données radio) jusqu'aux régions centrales (grâce aux données optiques).

Dans ce travail on s'intéresse d'abord aux données optiques. Les champs de vitesses montrent que la cinématique des étoiles est régulière dans les régions centrales, alors que les champs de vitesse du gaz présentent des perturbations. J'ai conduit une première analyse quantitative de ces champs de vitesse. On conclut alors que les pertubations cinématiques du gaz ionisé dans les régions centrales des galaxies de Seyfert suggèrent un lien entre la dynamique au centre de la galaxie hôte et les mécanisme d'alimentation du noyau actif. Nous avons enfin combiné les données radio et optique afin d'analyser la cinématique du disque galactique dans son ensemble. Bien que les méthodes de réduction soient différentes pour les données radio et optique, des techniques similaires d'analyse des champs de vitesse peuvent être appliquées aux deux types de données. J'ai développé un outil utilisant la décomposition en séries de Fourier de la vitesse radiale afin d'analyser les champs de vitesses du gaz atomique H I (données radio), du gaz ionisé et des étoiles (données optiques) de notre échantillon de galaxies actives et non-actives. Cette étude nous permet d'obtenir la courbe de vitesse à grande échelle jusque dans les régions centrales $\left(\mathrm{r}<20^{\prime \prime}\right)$, et ainsi de contraindre le potentiel gravitationnel $\mathrm{du}$ disque galactique dans son entier. Nous pourrons ainsi étudier les perturba- 
tions du gaz sur de grandes et petite échelles spatiales, comme les déviations par rapport à un mouvement circulaire, qui pourraient être directement liées à l'alimentation du noyau actif. 


\section{Acknowledgements}

Allow me now to name a few people I would like to thank for their help, support and encouragement during these past few years.

First of all, I am very thankful to my English and French supervisors Pr. Carole Mundell and Pr. Eric Emsellem and also to Dr. Pierre Ferruit (my "second" French supervisor) for all their help and support during my PhD. Without them, this PhD project would not have been done in the first place. In particular I thank them deeply for making possible for me to attend international conferences and scientific events during these three years. I want also to mention the two institutions that made this dual-award PhD possible: Liverpool John Moores University and the Ecole Normale Supérieure de Lyon. Financial support to this co-tutorship thesis has been provided by the Eurodoc program from the Rhône-Alpes region and by the French research ministry.

I want also to thank warmly Dr. Phil James and Dr. Niranjan Thatte for accepting to be the examiners of this thesis, and Pr. Chris Collins and Pr. Bruno Guiderdoni, to be part of my thesis "French" jury. The discussions we had during the viva, the French defence and afterwards were very interesting, and I'm looking forward to meeting them again!

Of course I will never forget the staff and students of the CRAL and ARI for all the (non) scientific moments we shared and the friendly atmosphere of both institutes.

In Lyon, I warmly thank the (ex-)Tiger team members and the "djeuns" of the observatory for supporting me (and my moods!) and for helping me all these years! I think especially to Arlette and Manu for the biscuits and the discussions at the coffee breaks. Many thanks to Manu and Xavier who were my successive officemates, and to Xavier, Aurélien, Nicolas, Manu, Arlette and Fabien for their help on computer issues, thanks to them I'm almost a geek now! My warmest thanks to all the "djeuns" : Emilie, Fabien, Guillaume, Xavier, Aurélien, Nicolas, Clémentine, Dylan, Emeline, PJ, Laure, Ivana, Mina, Johan... We had many great times all these years! Special thanks to Nicolas for the driving and the 'tartiflettes'!

I'm also very grateful to Emilie and Fabien for all the time we spent together. Thank you a lot for you friendship, for having listened to my problems and for having always supported me. 
I would like to thank the people from the ARI for their warmth welcome and all the nice time I had during the months I stayed there. I'd like to mention particularly Claire Thomas, Diego Black, Jim Allsopp, Dan Drown, Will Prestley, Danae Polychroni (and all the students), Maurizio Salaris, Phil James, Sue Percival, Dan Harman, Chris Simpsom, Chris Collins and Carole Mundell. I'm also thankful to André Brabin and Jane McCagh who helped me a lot with the administrative papers. Now as far as Clair and Derek (and Katie!) are concerned, let me thank you for your welcome, for all the movies and evenings we spent together, for the curries and all the nice moments I had living with you! Many thanks to Danae as well who took me in for a few weeks.

I'm of course very grateful to Gary Mamon and Philippe Mathias for helping me in my first steps in astrophysics research and for encouraging me to continue.

I'm also obliged to the Physics Department and the teaching team of the ENS for their advice and support, especially Bernard Castaing and Francesca Chillà. Many thanks to Hervé Gayvallet for his advice and for the complex organisation of the prépa agreg and thanks a lot to Jerome Cavoret for his infinite patience during the lab sessions! Many thanks to Fanfan and Yohan too.

In a more personal level, I'd like to thank Mélanie and the girls from the gym club for sharing so much fun and reminding me two hours per week that there's not only AGN in life! I also thank deeply my parents and all my family for having supported me during these long studies, helped me and for always believing in me. Many thanks to Momo as well for her help with the English!

Finally, the last but not the least, I cannot thank Mathieu enough for all his support, trust and love. Thank you for always being here for me. 


\section{Remerciements}

Je voudrais remercier ici les différentes personnes qui m'ont aidées et encouragées durant ces trois années de thèse.

Tout d'abord, je remercie mes deux directeurs de thèse : Pr. Carole Mundell et Pr. Eric Emsellem, ainsi que Dr. Pierre Ferruit pour toute l'aide qu'ils m'ont apportée durant ma thèse. Sans eux mon projet de thèse n'aurait jamais vu le jour en premier lieu. Je les remercie en particulier pour m'avoir donnée l'opportunité de participer à plusieurs conférences internationales. Je leur suis aussi très reconnaissante de m'avoir donnée la chance d'effectuer ma thèse en co-tutelle dans leurs instituts: 1'Astrophysics Research Insitute à Liverpool et l'Observatoire de Lyon. Je remercie aussi les deux universités dans lesquelles j'ai effectué ce travail de thèse: l'université John Moores de Liverpool et l'Ecole Normale Supérieure de Lyon. Cette thèse en co-tutelle a été en partie financée par la bourse Eurodoc de la région Rhône-Alpes et la bourse d'aide à la mobilité des doctorants du ministère de la recherche.

Je voudrais aussi remercier chaleureusement Dr. Phil James et Dr. Niranjan Thatte pour avoir accepté d'être les examinateurs de ma thèse, ainsi que les deux autres membres de mon jury: Pr. Chris Collins et Pr. Bruno Guiderdoni.

Je remercie toutes les personnes qui travaillent au CRAL et à l'ARI pour tous les moments scientifiques (ou non!) et amicaux que nous avons partagés. À Lyon, je voudrais surtout remercier l'(ex) équipe Tigre et les "djeuns" de l'observatoire pour m'avoir supportée pendant plus de trois ans! Un grand merci à Arlette et Manu pour les gateaux et les discussions de la pause café (et les barbecues!). Merci à Manu et Xavier pour avoir partagé mes bureaux successifs. Je voudrais aussi remercier Xavier, Aurélien, Nicolas, Arlette, Manu et Fabien pour leur aide précieuse sur les problèmes informatiques et logiciels. Je suis presque une geek maintenant grâce à eux! Merci aussi à tous les "djeuns": Emilie, Fabien, Guillaume, Xavier, Aurélien, Nicolas, Clémentine, Dylan, Laure, PJ, Emeline, Ivana, Mina... pour tous les moments passés ensemble dans ou en dehors de l'observatoire, pour les repas, fêtes et autres soirées. Un grand merci à Nico en particulier, pour les tartiflettes et le co-voiturage. Je voulais remercier tout spécialement Emilie et Fabien pour leur amitié. Merci beaucoup d'avoir écouté (patiemment!) tous mes problèmes et d'avoir toujours été là pour m’aider et partager de bon moments! 
Je tiens aussi à remercier toutes les personnes de l'Astrophysics Research Institute à Liverpool pour leur accueil et pour tout le bon temps que j'ai passé lorsque j'étais là-bas. Je remercie André Brabin et Jane McCagh de m'avoir aidée pour les démarches administratives. Merci aussi à Claire Thomas, Diego Black, Jim Allsopp, Dan Brown, Will Perstley, Danae Polychroni (et tous les étudiants), Maurizio Salaris, Phil James, Sue Percival, Dan Harman, Chris Simpson, Chris Collins et Carole Mundell. Je remercie aussi profondément Claire et Derek (et Katie!) qui m'ont accueillie dans leur maison. Merci pour les soirées et week-end, les restos et tous les bons moments passés ensemble. Merci aussi à Danae qui m’a recueillie à la fin de mon séjour à Liverpool.

Je voudrais aussi remercier Gary Mamon et Philippe Mathias qui ont accompagné mes premiers pas dans le monde de la recherche astrophysique et qui $\mathrm{m}^{\prime}$ ont toujours encouragée à continuer dans cette voie.

Je remercie aussi le laboratoire de physique de l'ENS et toutes les personnes de la préparation à l'agrégation, pour leurs conseils et encouragements. En particulier, je remercie Bernard Castaing, Francesca Chilla pour leur disponibilité. Je tiens aussi à remercier Hervé Gayvallet qui a toujours réussi à concilier l'emploi du temps et nos désirs, et Jérôme Cavoret pour sa patience face à toutes les questions que j'ai pu lui poser! Un grand merci aussi à tous mes collègues moniteurs, agrégé préparateurs ou autres, en particulier Fanfan et Yoann.

D'un point de vue plus personnel, je voudrais remercier Mélanie et les filles de la gym. Grâces à elles je passais deux heures par semaine merveilleuses et sans penser aux galaxies! Je remercie aussi profondément mes parents et toute ma famille pour m'avoir soutenue tout au long de mes (longues) études et pour toujours avoir cru en moi, quels que soient mes choix. Merci aussi à Momo pour la relecture de l'Anglais!

Enfin, je voulais profondément remercier Mathieu pour être à mes cotés. Merci pour ton soutient, ta présence et ton amour.

GAËLLE DUMAS 
The most exciting phrase to hear in science, the one that heralds new discoveries, is not 'Eureka!' (I found it!) but 'That's funny ...'

Isaac Asimov 


\section{Contents}

Declaration

iii

$\begin{array}{ll}\text { Abstract } & \mathbf{v}\end{array}$

Résumé vii

$\begin{array}{ll}\text { Acknowledgements } & \text { ix }\end{array}$

Remerciements $\quad$ xi

Contents xiv

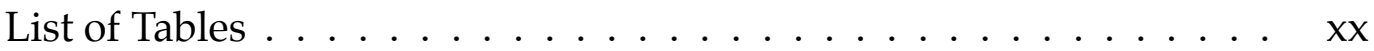

List of Figures . . . . . . . . . . . . . . . xxii

1 Introduction.

$\begin{array}{ll}\text { What are Active Galactic Nuclei? } & 1\end{array}$

1.1 An observational classification ............. 2

1.1.1 Radio-loud AGN .................. 2

1.1.2 Radio-quiet AGN . . . . . . . . . . . . . 5

1.2 Continuum spectrum . . . . . . . . . . . 7

1.3 Emission lines ...................... 9

1.4 Nature of the energy source . . . . . . . . . . . . . . 12

1.4.1 The super massive black hole paradigm . . . . . . . . . 12

1.4.2 Evidence for a central SMBH . . . . . . . . . . . 12

1.5 Unifying the AGN . . . . . . . . . . . . . . . . . . . 13

1.5.1 The standard model. . . . . . . . . . . . . . . . . 14 
1.5.2 Alternative models. . . . . . . . . . . . . 16

2 AGN-galaxy host connection. The fuelling issues. 19

2.1 Evolution of AGN . . . . . . . . . . . . . . . . . . 19

2.2 Fuelling the AGN . . . . . . . . . . . . . . 20

2.2.1 Galaxy interactions and mergers . . . . . . . . 22

2.2.2 Internal gravitational instabilities . . . . . . . . . 23

2.3 Two-dimensional kinematics of Seyfert galaxies . . . . . . . 25

2.3.1 Integral-Field Spectroscopy data . . . . . . . . . 27

2.3.2 Motivation of this thesis . . . . . . . . . . . 28

3 Sample selection, observations and data reduction 31

3.1 Sample selection . . . . . . . . . . . . . . . . . 31

3.1 .1 The parent sample ............... 31

3.1.2 The nuclear classification . . . . . . . . . . . 34

3.1.3 The VHIKINGS and SAURON/Seyfert samples . . . . . 38

3.2 Observation and data reduction . . . . . . . . . . . . . 44

3.2.1 VHIKINGS survey . . . . . . . . . . . . . . . 44

3.2.2 SAURON/Seyfert survey . . . . . . . . . . . 45

4 Results: the moment maps

4.1 The central regions of active and inactive galaxies . . . . . . 55

4.1.1 Stellar and ionised gas distribution . . . . . . . . 56

4.1.2 Stellar and ionised gas kinematics . . . . . . . . 58

4.2 Hi moment maps . . . . . . . . . . . . . . . . 59

4.2.1 H I morphology and environment . . . . . . . . . 60

4.2 .2 HI kinematics . . . . . . . . . . . . . . 60

4.3 summary.............................. 62

5 Kinematic analysis of the velocity fields 63

5.1 global kinematic major-axis orientation in the central regions . . 63

5.1.1 Stellar kinematics and the orientations of the line of nodes 65 
5.1.2 Global kinematic misalignments between stars and ionised gas . . . . . . . . . . . . . 6 67

5.1 .3 Statistical tests . . . . . . . . . . . . 68

5.2 Modelling the velocity fields . . . . . . . . . . . . . 70

5.2.1 The PyRing tool . . . . . . . . . . . . . 75

5.2 .2 Testing PyRing . . . . . . . . . . . . 77

5.2 .3 Conclusion from these tests. . . . . . . . . . . . . 89

5.3 Radial Variations of Kinematic Properties . . . . . . . . . . . 89

5.3.1 PA and rotation curve in the central regions . . . . . . . 89

5.3.2 H I velocity curves and position angles. . . . . . . . . . 99

5.3.3 Global variation of the kinematic parameters. . . . . . . 105

5.4 Summary and main results . . . . . . . . . . . . 108

$\begin{array}{lll}6 & \text { Discussion and Perspectives } & 111\end{array}$

6.1 Feeding the AGN and time-scales. . . . . . . . . . . . . . 111

6.2 Accretion rates and kinematic misalignments . . . . . . . . . 117

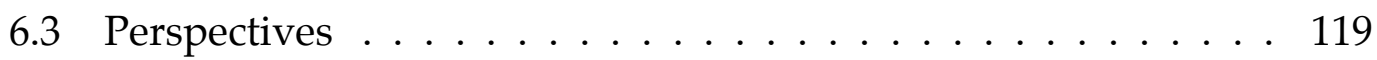

6.3.1 Harmonic analysis of velocity fields . . . . . . . . . 120

6.3.2 Other directions for further works. . . . . . . . . . . . 122

$\begin{array}{ll}\text { A The master sample } & 127\end{array}$

$\begin{array}{ll}\text { B The VHIKINGS sample } & 133\end{array}$

B.1 The DSS images . . . . . . . . . . . . . . . 133

B.2 The radio data . . . . . . . . . . . . . . 138

$\begin{array}{ll}\text { C SAURON moment maps } & 143\end{array}$

C.1 Stellar and ionised gas distribution and kinematic . . . . . . . 143

C.2 $h_{3}$ and $h_{4}$ maps . . . . . . . . . . . . . . 152

C.3 $\mathrm{H} \beta$ kinematic maps . . . . . . . . . . . . . . . . . 155

$\begin{array}{lr}\text { D HI moment maps } & 159\end{array}$ 
E Notes on individual galaxies.

E.1 NGC 1068/NGC 4548 pair . . . . . . . . . . . . . . . . 177

E.1.1 NGC1068 (M77) . . . . . . . . . . . . . 177

E.1.2 NGC $4548($ M91) . . . . . . . . . . . . . . . 178

E.2 NGC 3227/NGC3169 pair . . . . . . . . . . . . . . . . 179

E.2.1 NGC3227 . . . . . . . . . . . . . . . . . . . . 179

E.2.2 NGC3169 . . . . . . . . . . . . . . . 180

E.3 NGC 2655/NGC 4459 pair . . . . . . . . . . . . . . . 180

E.3.1 NGC $2655 \ldots \ldots \ldots \ldots$. . . . . . . . . . . 180

E.3.2 NGC $4459 \ldots \ldots \ldots \ldots$. . . . . . . . . . . . 181

E.4 NGC 3627/NGC5806 pair . . . . . . . . . . . . . . . 182

E.4.1 NGC3627 (M66) . . . . . . . . . . . . . . . . 182

E.4.2 NGC5806 . . . . . . . . . . . . . . . 182

E.5 NGC 4051/NGC5248 pair . . . . . . . . . . . . . . . 183

E.5.1 NGC $4051 \ldots \ldots \ldots \ldots$. . . . . . . . . . . . . 183

E.5.2 NGC5248 . . . . . . . . . . . . . . . . . 184

E.6 NGC 4151/NGC 2985 pair . . . . . . . . . . . . . . . . 185

E.6.1 NGC $4151 \ldots \ldots \ldots \ldots \ldots$

E.6.2 NGC $2985 \ldots \ldots \ldots \ldots$. . . . . . . . . . . 186

E.7 NGC 4579/NGC3351 pair . . . . . . . . . . . . . . . . . 187

E.7.1 NGC $4579($ M 58) . . . . . . . . . . . . . . 187

E.7.2 NGC3351 (M95) . . . . . . . . . . . . . . . 188

E.8 NGC 5194/NGC5055 pair . . . . . . . . . . . . . . . . . 189

E.8.1 NGC5194 (M51) . . . . . . . . . . . . . . . . 189

E.8.2 NGC5055 (M63) . . . . . . . . . . . . . . 189

E.9 NGC $6951 /$ NGC 5248 pair . . . . . . . . . . . . . . . . . 190

E.9.1 NGC $6951 \ldots \ldots \ldots \ldots$

E.10 NGC 3147/NGC 4041 pair . . . . . . . . . . . . . . . . . 191

E.10.1 NGC $3147 \ldots \ldots \ldots \ldots 1 . \ldots \ldots \ldots$

E.10.2 NGC $4041 \ldots \ldots \ldots 1 . \ldots \ldots 1$

Xviii 
E.11 NGC $4593 /$ NGC5383 pair . . . . . . . . . . . . . . . . . . 192

E.11.1 NGC $4593 \ldots \ldots \ldots$

E.11.2 NGC5383 . . . . . . . . . . . . . . . . . . . . . . 192

E.12 NGC 4939/NGC5364 pair . . . . . . . . . . . . . . . . . 192

E.12.1 NGC $4939 \ldots \ldots$. . . . . . . . . . . . . . . . . . . 192

E.12.2 NGC5364 . . . . . . . . . . . . . . . . . . . . . 193

E.13 NGC5427/NGC 2967 pair . . . . . . . . . . . . . . . . . 193

E.13.1 NGC5427 . . . . . . . . . . . . . . . . . . . . . 193

E.13.2 NGC 2967 . . . . . . . . . . . . . . . . . . . . . 193

E.14 NGC 4941/NGC3705 pair . . . . . . . . . . . . . . . . . . 193

E.14.1 NGC $4941 \ldots \ldots \ldots$. . . . . . . . . . . . . . . 193

E.14.2 NGC3705 . . . . . . . . . . . . . . . . . . . . 193

E.15 NGC 4639/NGC3162 pair . . . . . . . . . . . . . . . . . 194

E.15.1 NGC 4639 . . . . . . . . . . . . . . . . . . . . . 194

E.15.2 NGC3162 . . . . . . . . . . . . . . . . . . . . . 194

E.16 NGC $4258 /$ NGC 4527 pair . . . . . . . . . . . . . . . . . . 194

E.16.1 NGC 4258 (M106) . . . . . . . . . . . . . . . . . . 194

E.16.2 NGC $4527 \ldots \ldots$. . . . . . . . . . . . . . . . . . . 195

E.17 NGC 6814/NGC3963 pair . . . . . . . . . . . . . . . . . 195

E.17.1 NGC6814 . . . . . . . . . . . . . . . . . . . . . . 195

E.17.2 NGC3963 . . . . . . . . . . . . . . . . . . . . 195

$\begin{array}{ll}\text { Bibliography } & 196\end{array}$ 


\section{List of Tables}

1.1 Unification model of AGN. . . . . . . . . . . . . . . 14

3.1 Sample selection criteria. . . . . . . . . . . . . . . 32

3.2 Criteria for spectral classification. . . . . . . . . . 34

3.3 Properties of our sample. . . . . . . . . . . . . . . 43

3.4 Configuration properties. . . . . . . . . . . . . . . 45

3.5 Details of the exposures of our sample. . . . . . . . . . . 46

3.6 SAURON instrumental characteristic . . . . . . . . . . . . . 47

5.1 Results of the kinematic analysis of the stellar and gaseous components in the central regions. . . . . . . . . . 71

5.2 Estimation of the dynamical parameters. . . . . . . . . 78

5.3 Estimation of the dynamical parameters of the observed $\mathrm{H}$ I velocity field of NGC 5364. . . . . . . . . . . . . . 83

5.4 Estimation of the dynamical parameters of the observed stellar velocity field of NGC5055. . . . . . . . . . . . . 87

5.5 Effective radius . . . . . . . . . . . . . . . . 90

6.1 Eddington Ratios and Mass Accretion Rates. . . . . . . . . . . 116

A.1 Properties of the master sample . . . . . . . . . . . . 128

B.1 VLA observation parameters. . . . . . . . . . . . 139 


\section{List of Figures}

1.1 The radio-loud quasar 3C $273(\mathrm{z}=0.16)$, observed at $2 \mathrm{~cm}$ as part of a VLBA survey (Kellermann et al., 1998). . . . . . . . . . . 3

1.2 The FR-I radio galaxy 3C 465, corresponding to the elliptical galaxy NGC7720, observed with the VLA at 20 $\mathrm{cm}$ (from the 3CRR-Atlas of Leahy, Bridle, and Strom, http://www.jb.man.ac.uk/atlas/). . . . . . . . . 4

1.3 The FR-II radio galaxy Cygnus A, observed with the very large array (VLA) at $6 \mathrm{~cm}$ (Perley et al., 1984). The extent of the radio emission is about $140 \mathrm{kpc} . \ldots \ldots \ldots 5$

1.4 Schematic SED of radio-loud and radio-quiet AGN . . . . . . . 7

1.5 Optical spectra of various kinds of active galactic nuclei. . . . . 9

1.6 Example of diagnostic diagrams (Kewley et al., 2006) . . . . . 11

1.7 Unification scheme for the type 1 and 2 Seyfert galaxies . . . . 15

1.8 AGN component and spatial scales. . . . . . . . . . . . 17

2.1 QSO luminosity function. . . . . . . . . . . . . . . 21

2.2 Distribution of the quasars space density as a function of redshift. 21

2.3 OASIS optical layout. . . . . . . . . . . . . . . . . 26

3.1 Master sample galaxies properties. . . . . . . . . . . 33

3.2 Diagnostic diagrams. . . . . . . . . . . . . . . 35

3.3 Diagnostic diagrams for ambiguous galaxies. . . . . . . . . . 39

3.4 R-band Digital Sky Survey images of the SAURON sample galaxies. 41

3.5 Zone of rejection of the null hypothesis. . . . . . . . . . . . 42

3.6 Spectrum of NGC 5194 showing the spectral windows masked for the pixel-fitting iteration. . . . . . . . . . . . . . . 49

3.7 Contribution of the Hermite polynomials in the LOSVD profile. 50 
3.8 Examples of spectra and their corresponding fits for 4 galaxies.

3.9 Radial normalised profiles of the BLR . . . . . . . . . . 52

3.10 Example of contaminated spectrum of NGC 4051. . . . . . . . . 53

4.1 SDSS image of NGC 4151. The outer spiral arms are visible though very weak. . . . . . . . . . . . .

5.1 Comparison of observed and symmetrised velocity fields for NGC 2655. . . . . . . . . . . . . . . . .

5.2 Distribution of the differences between the photometric and the global stellar kinematics PAs. . . . . . . . . . . . 66

5.3 Kinematic misalignments between stars and ionised gas. . . . . 67

5.4 Cumulative fraction distributions. . . . . . . . . . . . 69

5.5 NGC5055 stellar velocity field divided in concentric rings. . . . 72

5.6 Projection of the galactic disk on the sky. . . . . . . . . 73

5.7 Observed and model velocity field of NGC5364 . . . . . . . 78

5.8 Comparison AIPS, GIPSY and Python tilted ring fits of a model

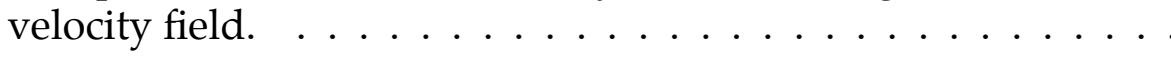

5.9 Comparison AIPS, GIPSY and Python tilted ring fit. Rotation velocity estimation.

5.10 Comparison AIPS, GIPSY and Python tilted-ring fits of an observed velocity field . . . . . . . . . . .

5.11 Comparison AIPS, GIPSY and Python tilted ring fit of an observation velocity field. Rotation velocity curve. . . . . . . . .

5.12 Comparison AIPS, GIPSY and Python tilted ring fit of an observational velocity field. Projected rotation velocity curve. . . . .

5.13 Comparison GIPSY and Python tilted ring fits of a SAURON ve-

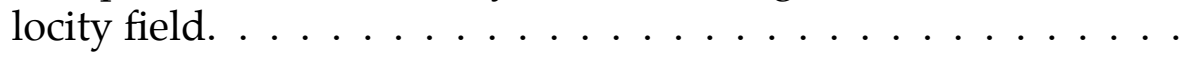

5.14 Comparison GIPSY and Python tilted ring fit. Rotation velocity estimation.

5.15 Comparison GIPSY and Python tilted ring fit on ionised gas

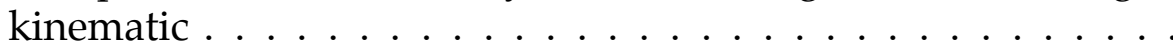

5.16aKinematic position angles of the stars and of the gas components. 91

5.16bKinematic position angles of the stars and of the gas components (continued). . . . . . . . . . . . . . . 
5.17 Distribution of the amplitude of variations of the stellar and the gaseous kinematic PAs. . . . . . . . . . . . . . . 93

5.18aRotation curve of the stars and of the gas components. . . . . . 97

5.18bRotation curve of the stars and of the gas components (continue). 98

5.19aRotation curve and kinematic position angles of the neutral gas components. . . . . . . . . . . . . 101

$5.19 \mathrm{bRotation}$ curve and kinematic position angles of the neutral gas components. (continued) . . . . . . . . . . . . . . 102

5.19cRotation curve and kinematic position angles of the neutral gas components. (continued) . . . . . . . . . . . . . 103

5.20 Comparison of H I stellar and ionised gas kinematics of NGC 2655104

5.21aRotation curve and kinematic position angles of all components. 106

$5.21 \mathrm{bRotation}$ curve and kinematic position angles of all components. (continued) . . . . . . . . . . . . . . 107

6.1 Diagnostic diagrams . . . . . . . . . . . . . . . 114

6.2 Differences between the global kinematic PAs of the stars and gas and amplitude of variations of the gaseous kinematic PAs as a function of the mass accretion rate. . . . . . . . . . . 115

6.3 Fuelling mechanisms and spatial scales. . . . . . . . . . . . . 119

6.4 Harmonic analysis of NGC 5055 velocity fields. . . . . . . . . . 123

6.5 Harmonic analysis of NGC 2967 and 5383 velocity fields. . . . . 124

B.1 R-band DSS images of the master sample galaxies. . . . . . . . 134

B.2 R-band DSS images of the master sample galaxies, continued. 135

B.3 R-band DSS images of the master sample galaxies, continued. . 136

B.4 R-band DSS images of the master sample galaxies, continued. . 137

C.1a SAURON maps for the Seyfert galaxies NGC1068 and NGC 3227. 144

C.1b SAURON maps for NGC 2655 and its control NGC 4459. . . . . . 145

C.1c SAURON maps for NGC3627 and its control NGC5806 . . . . . . 146

C.1d SAURON maps for NGC 4051 and its control NGC 5248. . . . . . 147

C.1e SAURON maps for NGC 4151 and its control NGC 2985. . . . . . 148

C.1f SAURON maps for NGC 4579 and its control NGC 3351. . . . . . 149 
C.1g SAURON maps for NGC5194 and its control NGC5055. . . . . . 150

C.1h SAURON maps for NGC 6951 and its control NGC 5248. . . . . . 151

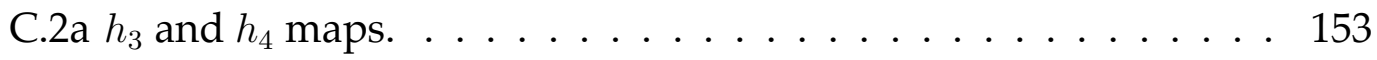

C.2b Fig. C.2a continued. . . . . . . . . . . . . . . . . . . . . 154

C.3a Velocity and velocity dispersion of $\mathrm{H} \beta$ emission lines. . . . . . 156

C.3b Fig. C.3a continued. . . . . . . . . . . . . . . . . . . . 157

D.1a H I moment maps. . . . . . . . . . . . . . . . . . . . . . . 160

D.1b Hi moment maps. . . . . . . . . . . . . . . . . . . . . . 161

D.1c Hi moment maps. . . . . . . . . . . . . . . . . . . . . . . 162

D.1d H I moment maps. . . . . . . . . . . . . . . . . . . . 163

D.1e H I moment maps. . . . . . . . . . . . . . . . . . . . . 164

D.1f H I moment maps. . . . . . . . . . . . . . . . . . . 165

D.1g H I moment maps. . . . . . . . . . . . . . . . . . 166

D.1h H I moment maps. . . . . . . . . . . . . . . . . . . . 167

D.1i H I moment maps. . . . . . . . . . . . . . . . . . . 168

D.1j H I moment maps. . . . . . . . . . . . . . . . . . . . . . 169

D.1k H I moment maps. . . . . . . . . . . . . . . . . . . 170

D.11 H I moment maps. . . . . . . . . . . . . . . . . . . . 171

D.1mH I moment maps. . . . . . . . . . . . . . . . . . . . 172

D.1n H I moment maps. . . . . . . . . . . . . . . . . . . . 173

D.1o H I moment maps. . . . . . . . . . . . . . . . . . . 174

D.1p H I moment maps. . . . . . . . . . . . . . . . . . . 175

D.1q Hi moment maps. . . . . . . . . . . . . . . . . 176 


\section{Chapter 1}

\section{Introduction. What are Active Galactic Nuclei?}

Galaxies are made up of stars, gas and dust and the galactic luminosity comes mainly from the thermal emission of these components. However, some galaxies in the Universe show unusual very bright nuclear regions, characterised by energetic phenomena (e.g. high ionisation regions, jets). Such nuclei are called Active Galactic Nuclei (AGN) and their hosts are active galaxies. The AGN include a wide range of different objects from the most powerful like the quasars to galaxies containing Low Ionisation Nuclear Emission Regions (referred as LINER galaxies in the following) at the low luminosity side and they share several properties, which define the AGN class:

- High Luminosity. The AGN produce a prodigious amount of energy, from about 1 to $10^{4}$ times the luminosity of a typical galaxy. The average bolometric luminosity emitted by the central regions of an active galaxy reaches $10^{44} \mathrm{erg} \mathrm{s}^{-1}$ for low luminosity AGN and more than $10^{48} \mathrm{erg} \mathrm{s}^{-1}$ for the most powerful objects. For comparison, the typical total luminosity of a field galaxy is about $10^{44} \mathrm{erg} \mathrm{s}^{-1}$. The AGN luminosity depends also on the redshift of the object. Luminosities of AGN at $z \simeq 2$ are about 100 times greater than for AGN in the local Universe (Krolik, 1999).

- Small Angular Size. This high luminosity seems to originate in a very small region in the centre of the host galaxy. However, this picture depends strongly on the wavelength. While X-ray images of active galaxies are mostly point-like, corresponding to the inner parsec, radio emission related to the AGN can extend over large spatial scales, sometimes beyond the galaxy itself (see Sec. 1.1.1).

- Broad Band Continuum. The integrated luminosity of a normal galaxy is mostly due to the stars, gas and dust components from the infra-red (IR) to the ultra-violet (UV) wavelengths. Emissions in radio (corresponding to atomic and molecular gas) and X-rays or $\gamma$-rays (hot gas, supernovae remnants) are less important. 
In the case of an AGN, the observed spectrum is almost flat from radio to $\gamma$-rays, with similar luminosity at all wavelengths. In some cases, the radio luminosity emitted by an AGN is more than a magnitude larger than for a normal galaxy, and in X-rays, the difference between AGN and non-active galaxies is even greater, reaching three or four orders of magnitude (Krolik, 1999). The weak local maxima emerging from the flat AGN spectrum allow to constrain the emission mechanisms, as a function of wavelength.

- Strong Emission Lines. In most cases, strong optical and IR emission lines are observed in the spectrum of AGN. The width of these emission lines can extend up to several thousands $\mathrm{km} \mathrm{s}^{-1}$ and such lines are often associated with high ionisation processes.

- Variability. The luminosity emitted by an AGN is also characterised by time variability, in some cases these fluctuations are observed on time scales of order of days (e.g. UV continuum) and even hours or minutes in the case of X-rays emission (Blandford et al., 1990). Because of such variations, the interpretation of the AGN spectra taken at different times may be difficult, since they may show different characteristics at different epochs.

Despite these common characteristics, AGN form a heterogeneous group, with different physical properties, especially in terms of the nature of the emitted radiations. The observed differences are used to classify the different types of AGN. In the following section, I describe one of such classifications.

\subsection{An observational classification}

The difference of luminosity in radio wavelengths leads to the main classification of the AGN, depending on the radio-to-optical flux ratio. Different values of this ratio can be set to define the radio-loudness of an AGN, depending on the considered radio and optical wavelengths. Usually is used the ratio $\mathrm{R}=\mathrm{L}_{5 G H z} / \mathrm{L}_{B}$ where $\mathrm{L}_{5 \mathrm{GHz}}$ is the flux at $5 \mathrm{GHz}$ and $\mathrm{L}_{B}$ is the optical flux in the $\mathrm{B}$-band centred on $4400 \AA$. Then, if $\mathrm{R} \gtrsim 10$ the $\mathrm{AGN}$ is called radio-loud while it is classified as radio-quiet if $\mathrm{R} \lesssim 10$ (Kellermann et al., 1989; Stocke et al., 1992). The majority of AGN are radio-quiet, only $10 \%$ being radio-loud. In this section, I describe the different properties of the radio-loud and radio-quiet AGN.

\subsubsection{Radio-loud AGN}

The radio-loud nuclei are found in giant elliptical galaxies and in some distant quasars. In such objects, extended jets or lobes are observed in radio emission. Such emission is due to relativistic electrons in the hot material via synchrotron 


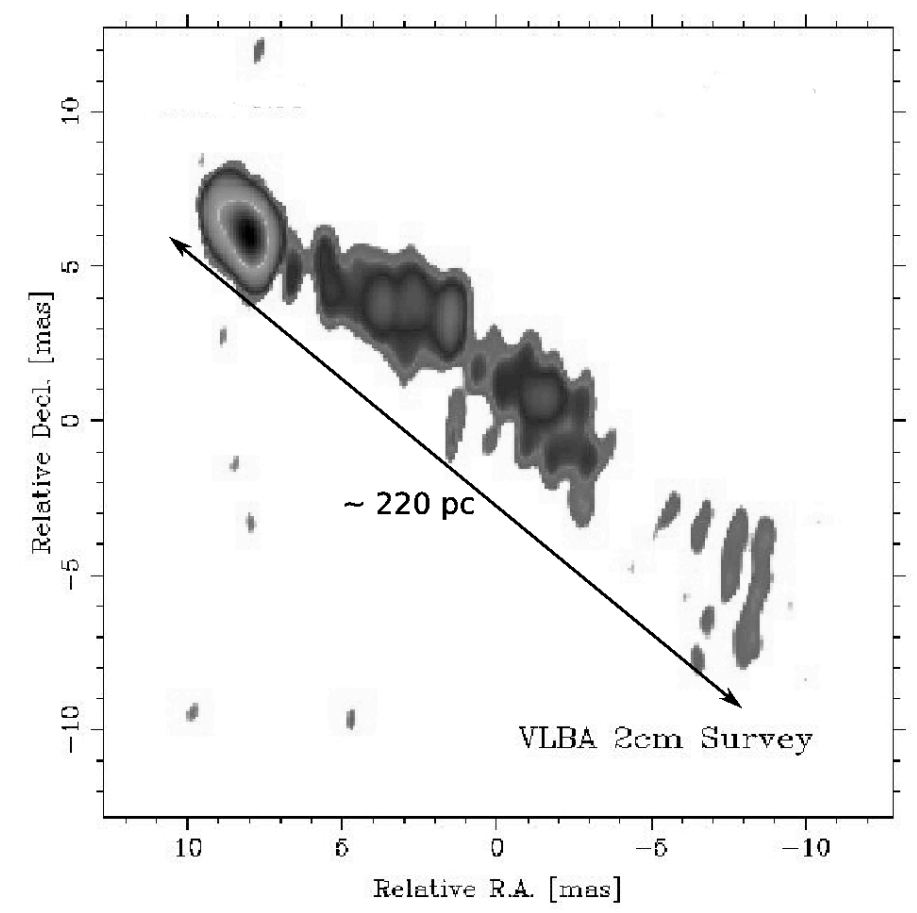

Figure 1.1: The radio-loud quasar 3C $273(\mathrm{z}=0.16)$, observed at $2 \mathrm{~cm}$ as part of a VLBA survey (Kellermann et al., 1998).

mechanisms (see Sec. 1.2). The main classes of radio-loud AGN are radiogalaxies, quasars and BL Lac objects:

\section{- Radio-galaxies.}

Galaxies in the local Universe hosting an AGN with high radio luminosity are called radio-galaxies. Mostly they are elliptical galaxies. Different types of radio-galaxies are defined depending on the predominant source of the radio emission.

Core-dominated objects present the bulk of radio emission in unresolved regions (in the scale of the arcsecond) in the central parts of the host galaxies. They present flat and variable radio spectrum. Recently, high spatial resolution observations, via e.g. very long baselines interferometer (VLBI), allow to resolve these compact radio-source. Jets of a few hundred of parsecs are often associated with such objects, as shown in the fig. 1.1.

In the lobe-dominated radio-galaxies on the other hand, the radio emission originates predominantly from two large lobes of several hundred of kpc in extent. In these lobes, radio emission presents a steep spectrum. Within these lobe-dominated class, a sub-classification has been defined by Fanaroff \& Riley (1974). This classification depends on the morphology of the radio lobes and the optical/UV emission properties. 


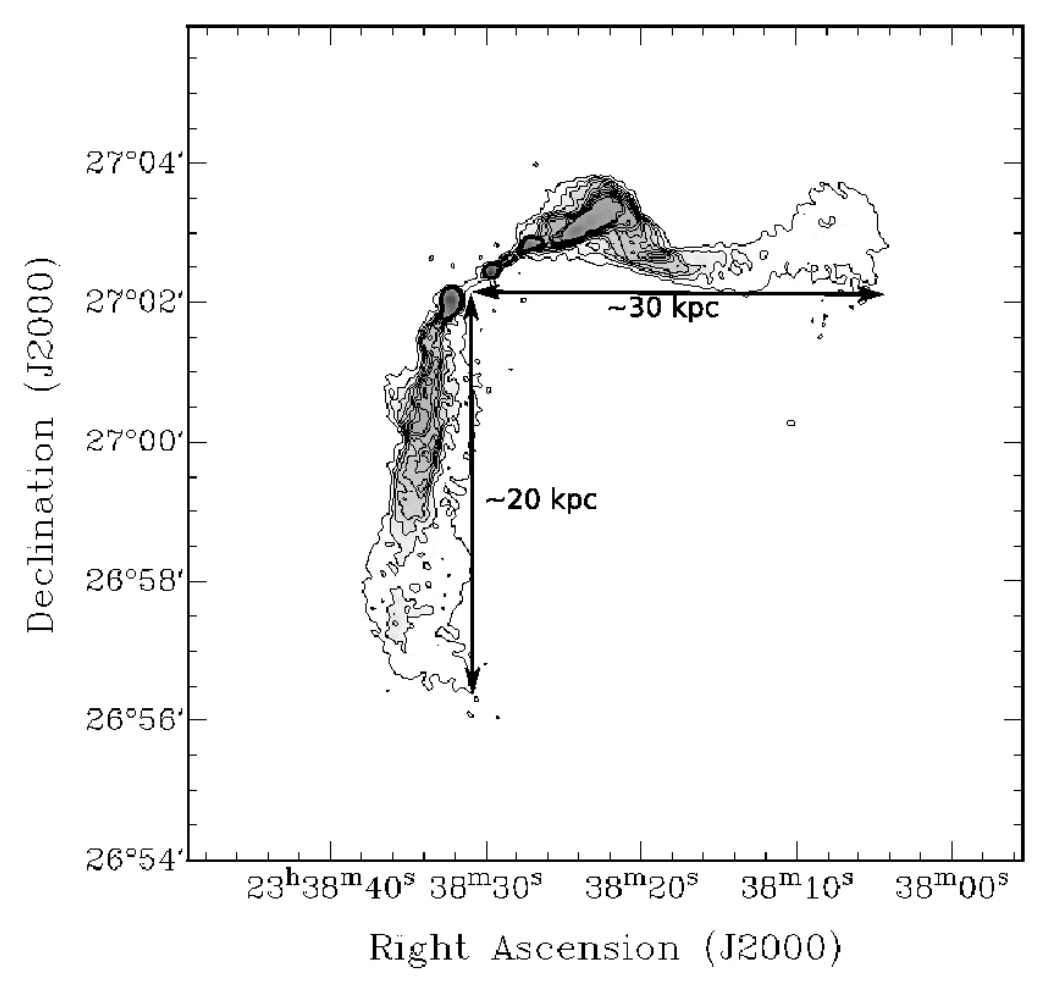

Figure 1.2: The FR-I radio galaxy $3 C 465$, corresponding to the elliptical galaxy NGC 7720, observed with the VLA at $20 \mathrm{~cm}$ (from the 3CRR-Atlas of Leahy, Bridle, and Strom, http:/ / www.jb.man.ac.uk/atlas/).

FR-I radio-galaxies display two-sided radio jets extending from the host galaxy to $\sim 1 \mathrm{kpc}$. At the end of such jets, extend radio lobes. Fig. 1.2 shows the FR-I radio galaxy 3C 465. The jets are asymmetric, the southeast jet being weaker than the north-west one. The radio lobes are sharply bent with respect to the jet direction. Optical and UV emission of FR-I radio-galaxies display only narrow lines, they are then all narrow lines radio-galaxies (NLRG).

FR-II radio-galaxies are more powerful than their FR-I counterparts and display larger radio structures. Collimated jets, associated with ultra relativistic motions, arise from the nucleus at several kiloparsec scales. At the ends of these jets, strong radio lobes extending for several $\mathrm{kpc}$ are observed. Cygnus A is the archetype of FR-II radio-galaxies. Fig. 1.3 shows the radio emission at $6 \mathrm{~cm}$ observed with the Very Large Array (VLA) by Perley et al. (1984). The large radio lobes are visible at the end of fainter collimated jets. The bright spot in the centre corresponds to the host galaxy nucleus. The total extent of the radio emission of Cygnus A is about $140 \mathrm{kpc}$. The UV/optical spectra of FR-II radio-galaxies present either broad and narrow lines or only narrow emission lines, these objects are then called broad line radio-galaxies (BLRG) and narrow line radio-galaxies (NLRG), respectively. 


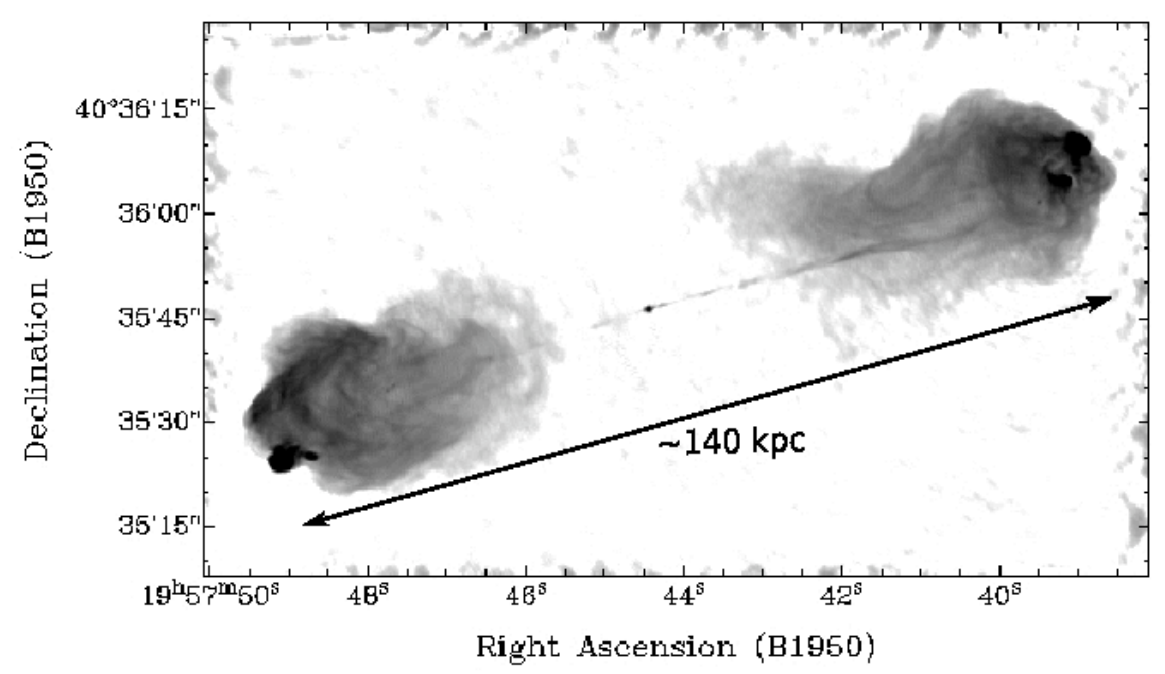

Figure 1.3: The FR-II radio galaxy Cygnus A, observed with the very large array (VLA) at $6 \mathrm{~cm}$ (Perley et al., 1984). The extent of the radio emission is about $140 \mathrm{kpc}$.

- Radio-loud quasars. The quasars are high redshift objects associated with galactic formation and interaction. They were discovered in the 60s with the development of radio-astronomy. Since the optical counterparts of the strong observed radio sources appeared point-like, similar to stars, these objects were named Quasi-Stellar Radio Sources or Quasars. Later, similar objects with lower radio emission were found. These radio-quiet quasars are called Quasi-Stellar Objects (QSOs). About only 10\% of all the quasars are radio-loud. Their spectra contain absorption lines which were soon associated with the Hydrogen Balmer lines, redshifted due to the distance of these objects. The nucleus of a quasar is very luminous, with $L_{b o l}>10^{46}$ ergs s$^{-1}$ which corresponds to more than 100 times the luminosity of a normal field galaxy. Radio-loud quasars present onesided jets associated with super-luminal motions. Fig. 1.1 presents the quasar 3C 273, observed at $2 \mathrm{~cm}$ (Kellermann et al., 1998). The one-sided radio jet extends out to about $220 \mathrm{pc}$.

- Blazars: BL Lac objects, Optically Violent Variable objects. These peculiar objects display rapid time variations of high amplitude, the flux can vary by $10 \%$ or more on a year at all wavelengths (Wiita, 2006). Their spectra seem to be dominated by emission from relativistic jets and present neither emission nor absorption lines (see top left panels of Fig. 1.5).

\subsubsection{Radio-quiet AGN}

Radio-quiet and radio-loud active galaxies present similar IR, optical, UV and X-rays properties (Neugebauer et al., 1986; Sanders et al., 1989; Steidel \& Sar- 
gent, 1991). However, radio-quiet objects differ from their radio-loud counterparts in term of radio emission. Radio-quiet AGN do not show large radio structures, although parsec-scale jets can be observed in some cases. The classification of radio-quiet AGN depends essentially on their optical/UV spectral properties.

- Seyfert galaxies. The Seyfert galaxies are nearby spiral or irregular galaxies. They are low luminosity AGN with a nuclear magnitude $\mathrm{M}_{n u c} \gtrsim-21.5$, corresponding to a bolometric luminosity less than $10^{45} \mathrm{erg} \mathrm{s}^{-1}$. They were the first AGN to be discovered by Seyfert (1943) who noted their characteristics (broad and strong emission lines, unresolved nuclei, high luminosity, high mass) which differentiate them to normal galaxies. There are two distinct subclasses of Seyfert galaxies based on the presence or absence of broad emission lines:

Seyfert 1 galaxies display broad permitted emission lines with full width at half maximum $(\mathrm{FWHM}) \sim 1000,10000 \mathrm{~km} \mathrm{~s}^{-1}$. The regions emitting these broad lines are called broad line regions (BLR). Narrow permitted and forbidden emission lines are also observed. They are emitted from a different region, the narrow line region (NLR). Both low ionisation lines (e.g. $\mathrm{H} \alpha, \mathrm{H} \beta$ ) and high ionisation lines (e.g. [O III] $\lambda \lambda 49959,5007$ ) are present in the BLR and NLR. These broad and narrow emission lines are discussed in detail in Sec. 1.3.

Seyfert 2 galaxies are less luminous than Seyfert 1's. The spectra of Seyfert 2 galaxies is similar to the ones of the Seyfert 1's, the only difference being the absence of the broad permitted lines.

Later, Osterbrock (1981) defined type 1.5, 1.8 and 1.9 Seyfert galaxies for AGN with characteristics of both Seyfert 1 and Seyfert 2 galaxies.

- Radio quiet quasars. The majority of quasars $(\sim 90 \%)$ are radio-quiet. They are commonly called QSO (quasi stellar objects). As for their radioloud counterparts, these objects are very luminous, $\mathrm{M}_{n u c} \lesssim-21.5$, and are generally spatially unresolved. The optical spectra of these objects are very similar to the Seyfert 1 galaxies spectra, except that stellar absorption lines are not detected and that narrow emission lines are very weak.

- LINER galaxies. Heckman (1980) identified low ionisation nuclear emission line regions (LINER) galaxies, classified now as active galaxies with very low nuclear luminosity (about $10^{39} \mathrm{erg} \mathrm{s}^{-1}$ ). The optical spectra of the LINER galaxies are similar to those of the Seyfert 2's, with relatively high strength of the low ionisation lines (e.g. $\mathrm{H} \alpha, \mathrm{H} \beta$ ).

Both radio-loud and radio-quiet AGN display important features in their continuum spectra and in some cases strong emission lines. Their properties allow to constrain the physical conditions of the AGN. In the two following sections, I then review the main characteristics of the continuum and emission lines emitted by AGN. 


\subsection{Continuum spectrum}

A normal galaxy emits nearly all its luminosity within a few decades in frequency, from IR to UV wavelengths. In most cases, the spectrum of an active galaxy looks spectacularly different. AGN emit within a wide range of wavelengths with almost equal power on several decades in frequency, from mid-infrared to hard X-rays. The spectral energy distribution (SED) of AGN displays different features on top on this flat continuum. These features are good indicators of the emission mechanisms. In particular, continuum studies allow to constrain the importance of thermal and non-thermal emission processes in various frequency bands. In this section, I review the main features of the AGN continuum spectrum from X-rays to radio wavelengths. Fig. 1.4 displays schematic SEDs of radio-quiet and radio-loud AGN. The different parts of the spectrum are represented with the associated radiation processes.

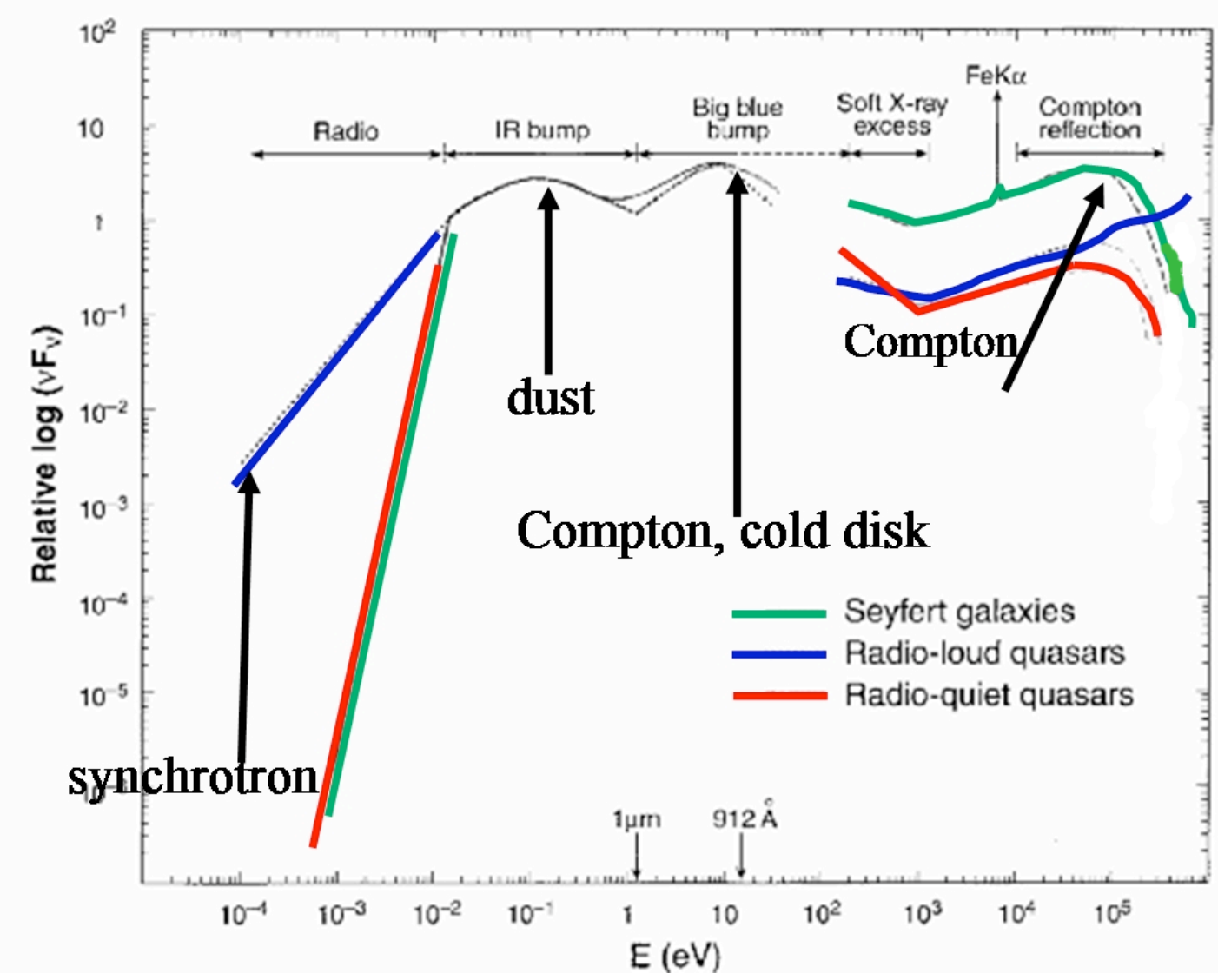

Figure 1.4: Schematic SED of radio-loud and radio-quiet AGN. Blue, red and green lines correspond to the characteristics of radio-loud quasars, radio-quiet quasars and Seyfert galaxies, respectively. The black lines correspond to SED components which are similar for these three types of AGN (from Koratkar \& Blaes, 1999). 
- X-rays and $\gamma$-rays emission. Xrays emission extends from $\sim 0.1 \mathrm{keV}$ up to $\sim 300 \mathrm{keV}$. To a first approximation, the dominant component is a power law extending from $\sim 1 \mathrm{keV}$ up to $\sim 100 \mathrm{keV}$. Beyond, an exponential cut-off is observed, from $\sim 80$ to $\sim 300 \mathrm{keV}$ (Risaliti \& Elvis, 2004). Rapid fluctuations (of the order of hours or minutes) are observed in the X-rays and $\gamma$-rays spectra of AGN. Such fluctuations are evidence for emitting regions in the innermost parts of the galaxy. Several radiation mechanisms may contribute to the production of X-rays and $\gamma$-rays continuum, such as thermal (bremsstrahlung) and non-thermal (inverse-Compton scattering) processes (Krolik, 1999). Synchrotron and synchrotron self Compton mechanisms may potentially emit such radiations but are not the predominant processes.

- UV/optical emission. The UV/ optical continuum emission is dominated by the Big Blue Bump (BBB) which extends from the NIR $(\sim 1 \mu \mathrm{m})$ to the $\mathrm{UV}$ and in some cases to the soft X-rays. This component is thought to be associated with thermal emission of material at a characteristic temperature of $\sim 10^{5} \mathrm{~K}$, located in an optically thick accretion disk surrounding the central supermassive black hole (Shields, 1978; Malkan \& Sargent, 1982; Laor \& Netzer, 1989). However, models of accretion in an optically thick disc failed to reproduce the UV/optical spectrum of AGN. Recent models of two-phase accretion disk take into account bremsstrahlung radiations of free electrons in optically thin medium as well as the black body emission from the optically thick regions to fit the observed UV / optical continuum (Collin-Souffrin et al., 1996; Nayakshin \& Melia, 1997).

- IR emission. AGN IR spectrum is formed by a broad continuum between 2 and $100 \mu \mathrm{m}$, of the same luminosity as the optical/UV emission, with a sharp drop-off for $\lambda>100 \mu \mathrm{m}$ (Haas et al., 2003). Thermal emission from dust heated by the UV-optical continuum cannot be the unique source of the IR continuum in AGN and non-thermal emission produced by synchrotron mechanism is important, in particular in far infra-red (FIR) emission of radio-loud quasars (Risaliti \& Elvis, 2004). Similar time variations as the UV/ optical continuum are observed in some cases, with a significant time delay. This delay gives the spatial separation between the UV/ optical and IR emitting regions.

- Radio emission. As explained earlier, the fraction of luminosity in radio wavelength depends on the type of AGN (see Sec. 1.1.). Generally, AGN emit less flux in radio than in the UV/optical frequencies, by 3 to 6 orders of magnitude. In the case of radio-loud AGN though, radio luminosity can be as powerful as optical emission. Then, unlike all other wavebands, the radio continuum is emitted in regions of large spatial extent (radio lobes and jets) in addition to emission in the central unresolved region (see Sec. 1.1.1). In some cases, the AGN radio emission is also linearly polarised up to of a few 10 s of percent. Such strong polarisation cannot be produced by thermal mechanisms and synchrotron 
emission by relativistic electrons seems to be the predominant process in the production of AGN radio continuum (Krolik, 1999).

\subsection{Emission lines}
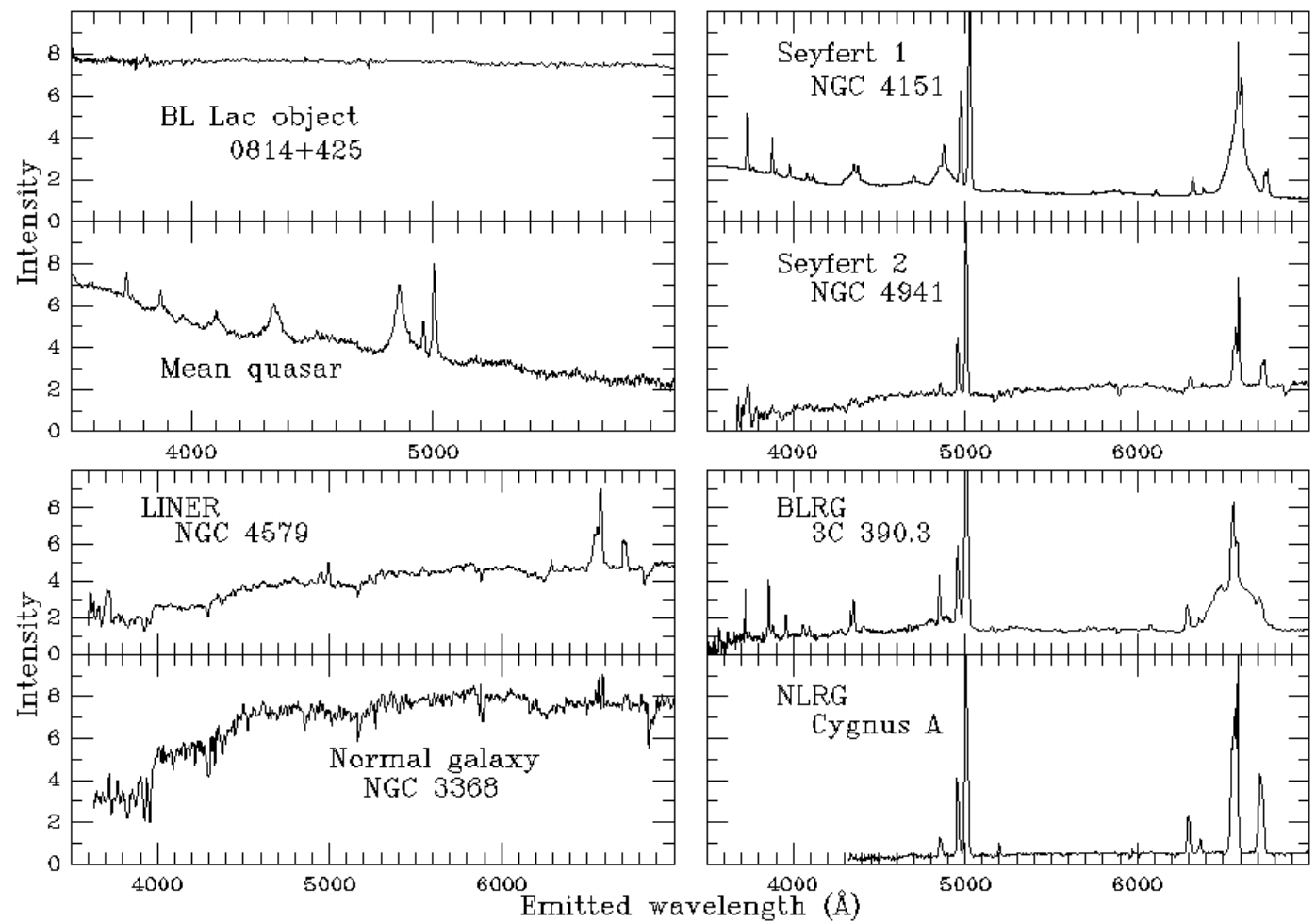

Figure 1.5: Optical spectra of various kinds of active galactic nuclei.

Strong emission lines observed in IR, optical, UV and even X-ray wavelengths are one of the dominant features of the spectrum of all AGN, except for the particular BL Lac objects (see Fig. 1.5, top left panel). Such emission lines are not observed in the spectra of non-active galaxies, as shown in the bottom left panel of Fig. 1.5. The properties of the AGN emission lines constrain the physical condition of the ionised gas in different regions of the AGN, such as the temperature, density and degree of ionisation, and shed lights on the dynamical mechanisms at works in these regions. In this section, I describe the characteristics of the optical/UV emission lines. AGN emission lines can be separated in two types. Some objects exhibit lines with broad wings as well as narrower profiles, while for other objects, only narrow lines are observed. 


\section{- Broad emission lines.}

Any emission line presenting width values from $1000 \mathrm{~km} \mathrm{~s}^{-1}$ up to 10000 $\mathrm{km} \mathrm{s}^{-1}$ at full width at half maximum (FWHM) is called broad line. Typical values of the FWHM of such broad lines are around $5000 \mathrm{~km} \mathrm{~s}^{-1}$. These lines are detected in quasars, type 1 Seyfert galaxies and broad line radio-galaxies (BLRG) as shown in Fig. 1.5. Only permitted lines are detected with such broad components, and among the several observed broad lines, the most important are the hydrogen lines (e.g. Ly $\alpha \lambda 1216, \mathrm{H} \beta \lambda 4861, \mathrm{H} \alpha \lambda$ 6563), the helium lines (e.g. HeII $\lambda 1640$ ) and the carbon lines (e.g. CIV $\lambda 1549)$. The broad lines are emitted by high velocity clouds in regions close to the central engine, generally spatially unresolved, called broad line regions (BLR). The strength of the broad emission lines vary with time and these fluctuations are correlated with the AGN continuum fluctuations. The time delay between the continuum and broad line variations can be related to the size of the BLR, which typically extends from 0.01 to $0.1 \mathrm{pc}$ from the central continuum source.

The line profiles and relative strengths allow to probe the physical properties of the BLR. Since no broad forbidden line is observed, the density in the BLR is very high $\left(\mathrm{n}_{e} \geq 10^{9} \mathrm{~cm}^{-3}\right)$. Given that the typical temperature in the BLR is $10^{4} \mathrm{~K}$, the width of the broad emission lines cannot be explained by pure thermal motions. The broad width of these lines is then generally attributed to Doppler effect due to the motions of each line-emitting clouds.

\section{- Narrow lines.}

Permitted lines listed above and forbidden lines such as [O II] $\lambda 3727$, [O III] $\lambda \lambda 4959,5007,[\mathrm{~N}$ II] $\lambda \lambda 6548,6583$ or [O I] $\lambda \lambda 6300,6364$ present narrow components, with FWHM less than $1000 \mathrm{~km} \mathrm{~s}^{-1}$, in the AGN spectra. The forbidden lines, especially [O III] $\lambda 5007$, are stronger than the permitted lines (except for $\operatorname{Ly} \alpha$ ), unlike in $\mathrm{H}$ II regions. In the case of Seyfert 2 galaxies, narrow line radio-galaxies (NLRG) and LINER galaxies, only narrow emission lines are observed, the broad emission lines are not detected (see Fig. 1.5). These narrow lines are emitted in spatially extended regions called the narrow line regions (NLR) of lower density than the $\operatorname{BLR}\left(n_{e} \geq 10^{3}-10^{6} \mathrm{~cm}^{-3}\right)$ and with temperatures between 10000 and $25000 \mathrm{~K}$.

The relative strength of narrow emission lines are used to differentiate high and low ionisation processes and then to distinguish between HII regions, LINER and Seyfert galaxies. Diagnostic diagrams with two pairs of lines have then been constructed to distinguish between blackbody in HII regions and high ionisation spectra of AGN. Several diagnostic lines can be used, in the optical wavelengths (e.g. [O III] $\lambda 5007 / \mathrm{H} \beta \lambda 4861, \quad[\mathrm{~N}$ II] $\lambda 6583 / \mathrm{H} \alpha \lambda 6563$ [S II] $\lambda \lambda 6717,6731 / \mathrm{H} \alpha \lambda 6563)$, UV (e.g. CIV $\lambda 1549 / \operatorname{Ly} \alpha \lambda 1216, \mathrm{~N} v \lambda 1240 / \mathrm{HeII} \lambda 1640)$ and IR (e.g. [Ne V] $\lambda 14$ $\mu \mathrm{m} /[\mathrm{Ne}$ II $] \lambda 12.8 \mu \mathrm{m},[\mathrm{O} \mathrm{IV}] \lambda 26 \mu \mathrm{m} /[\mathrm{Ne} \mathrm{II}] \lambda 12.8 \mu \mathrm{m},[\mathrm{Si} \mathrm{VI}] \lambda 1.962 \mu \mathrm{m} / \mathrm{Pa} \alpha)$. Fig. 1.6 shows examples of diagnostic diagrams (Kewley et al., 2006) with the limits of the different activity classes: Seyfert, LINER or HII galaxies. 
In general, narrow lines emission is strongly anisotropic. NLR present usually elongated shapes, known as the ionisation cones. Inside this feature, emission line ratios are characteristic of highly ionised gas, while outside the cone, they indicate that the gas is ionised by the stars. The ionisation cone can extend from $0.5 \mathrm{pc}$ up to a few $\mathrm{kpc}$, and can be either one-sided or two-sided. The axis of the cone is not correlated with the axis of rotation of the host galaxy, but is generally aligned with the radio emission, if it exists (e.g. Schmitt et al., 2003).

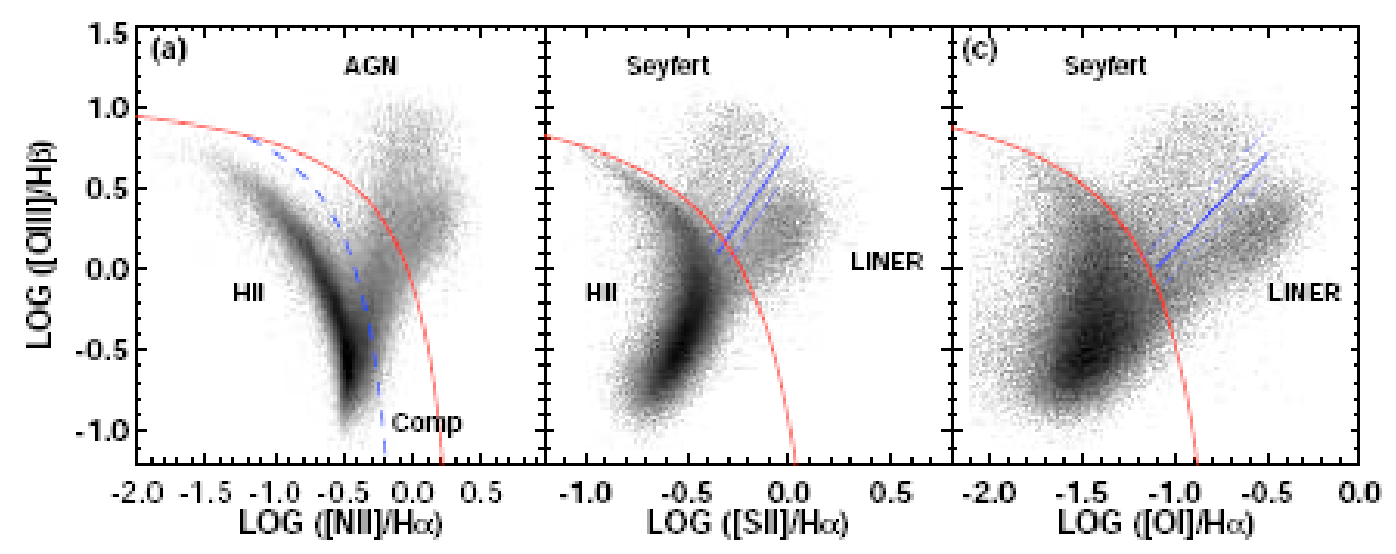

Figure 1.6: Example of diagnostic diagrams (Kewley et al., 2006). $[\mathrm{O}$ III] $\lambda 5007 / \mathrm{H} \beta \lambda 4861$ versus [N II] $\lambda 6583 / \mathrm{H} \alpha \lambda 6563$ diagnostic diagram is displayed in the left panel, [O III] $\lambda 5007 / \mathrm{H} \beta \lambda 4861$ versus [S II] $\lambda 6731 / \mathrm{H} \alpha \lambda 6563$ in the middle panel and [O III] $\lambda 5007 / \mathrm{H} \beta \lambda 4861$ versus [O I] $\lambda 6364 / \mathrm{H} \alpha \lambda 6563$ in the right panel. The Kewley et al. (2001) extreme starbust line, the Kauffmann et al. (2003) pure star forming line and the Kewley et al. (2006) classification line are shown as red solid, blue dashed and blue solid lines, respectively. Galaxies lying below the extreme starbust and the pure star forming lines are H II galaxies, while AGN lie above the extreme starbust line. Composite galaxies, between H II and AGN, are situated between the extreme starbust and the pure star forming lines (left panel). The Kewley et al. (2006) classification lines are used to separate the Seyfert galaxies from the LINER galaxies (middle and right panels)

Both in the BLR and NLR, high-ionisation lines are strong (e.g. [O III] $\lambda \lambda 4959,5007$ or CIV $\lambda 1549$ ). These lines require too much energy to be produced by thermal or shock excitation. Moreover, the time delay between the time fluctuations of the AGN continuum and those of the broad emission lines may correspond to the travel time of the ionising photons between the central source and the BLR clouds, while the shape, extent and orientation of the NLR support the existence of a collimated ionising radiation arising from the central engine. All these observational facts support the scenario where both broad and narrow emission lines are produced by photoionisation of the ISM by the AGN continuum. While close to the central source, hard X-ray photons fully ionise the gas, an extended partially ionised region exists between this ionised region and the neutral gas. In this regions, the gas is ex- 
cited by soft X-ray and far-UV photons. Lower excitation ions, such as [O I] or [S II] form in these regions. The size and ionic abundance of such partially ionised regions depends strongly on the photoionisation model (Oliva et al., 1999; Groves et al., 2004).

\subsection{Nature of the energy source}

The physical characteristics (high luminosity, small spatial scales, polarisation) and spectral properties (broad emission lines, radio emission) of AGN are evidence that the active galaxies host an energy source in addition to the thermal source provided by the stars. Since the different classes of active galaxies present several similar properties, it is now widely thought that the different types of AGN represent probably the same physical phenomenon, at different epochs and observed with different parameters. The current paradigm for the power source of the nuclear activity is the accretion of material onto a central super-massive black hole (Rees, 1984, SMBH,). In this section, I describe the $\mathrm{SMBH}$ model and the observational evidence for this central object.

\subsubsection{The super massive black hole paradigm}

A black hole more massive than $10^{6} \mathrm{M}_{\odot}$ is called a super-massive black hole $(\mathrm{SMBH})$. The energy released by material falling onto a black hole is about one order of magnitude larger than during the thermonuclear fusion of hydrogen (Lynden-Bell, 1969). Accretion of material onto a SMBH is then an efficient way to produce the prodigious energy observed in AGN. Very close to the SMBH, the accreted gas would have lost almost all its angular momentum. Therefore, it seems very likely that material falls onto the SMBH via a flat rotationallysupported disk, the accretion disk. In this accretion disk, the material loses its remaining angular momentum and fall onto the $\mathrm{SMBH}$, releasing the gravitational energy in the form of radiations or jets. Although direct evidence of the presumed $\mathrm{SMBH}$ surrounded by an accretion disk remains impossible with the current observational facilities, many observational properties of the central regions of AGN support the existence of a $\mathrm{SMBH}$.

\subsubsection{Evidence for a central SMBH}

The first argument supporting that AGN are powered by $\mathrm{SMBH}$ is the mass of the unresolved central object, constrained by the Eddington limit. The gravitational force on the gas emitting the AGN luminosity must exceed the radiation pressure, in other words, the AGN luminosity $L_{A G N}$ must not exceed the Eddington luminosity $L_{E d d}: L_{A G N}<L_{E d d} \simeq 3.310^{4} \frac{M}{M_{\odot}}$. Then, $M_{C M O}>810^{5} M_{\odot} \frac{L_{A G N}}{10^{44} \mathrm{ergs}^{-1}}$, where $M_{C M O}$ is the mass of the central massive 
object. Therefore, for an AGN emitting $10^{45} \mathrm{erg} \mathrm{s}^{-1}$, a mass of at least $10^{7} \mathrm{M}_{\odot}$ is concentrated within a region of small size, constrained by the time variations of the X-ray continuum. More evidence for the existence of a SMBH in the centre of $\mathrm{AGN}$ is provided via observations of these central regions:

- The widths of the broad emission lines, interpreted in terms of velocity of gas in the BLR, and the stellar and gaseous velocity dispersions in the central regions require a deep gravitational potential produced by a high mass concentration.

- Observations of $\mathrm{H}_{2} \mathrm{O}$ and $\mathrm{OH}$ masers in the central regions of AGN correspond to material in Keplerian motion around a point-like mass of more than $10^{6} \mathrm{M}_{\odot}$. In the Seyfert 2 galaxy NGC 4258, Miyoshi et al. (1995) constrained the central mass to be $\sim 3.610^{7} \mathrm{M}_{\odot}$ within 0.13 pc via VLBA maser observations.

- The strongest dynamical evidence for SMBH are certainly in our Galaxy, where measurements of stellar proper motions in the Galactic centre constrained the central mass to be of 3-4 $10^{6} \mathrm{M}_{\odot}$ (Genzel et al., 2000; Schödel et al., 2003).

- Rapid X-rays continuum fluctuations in the AGN spectra imply that the central mass is about $10^{6} \mathrm{M}_{\odot}$ for typical AGN. Such masses correspond to a Schwarzschild radius of $10^{-7}$ pc.

- The time delay between the continuum and broad emission lines variations is very likely related to the kinematics and geometry of the BLR. In particular, the size of the BLR and the mass enclosed within it can be constrained from this delay. This reverberation mapping of the BLR (Blandford \& McKee, 1982) reveals then central masses from $10^{7}$ to $10^{9}$ $\mathrm{M}_{\odot}$ enclosed within a region between 1 and a few tens of light-days in size (Peterson et al., 2004; Kaspi et al., 2005).

- Double peaked emission lines (e.g. $\mathrm{H} \alpha$, X-ray $\mathrm{K} \alpha$ iron line) are strong evidence for emission produced in a geometrically thin disk structure surrounding the central SMBH.

\subsection{Unifying the AGN.}

The model of accretion onto a SMBH seems to explain well most of the characteristics of the AGN, and numerous studies focused on unifying the different types of AGN with a few physical parameters. Reviews of the standard unification scheme can be found in Antonucci (1993) and Urry \& Padovani (1995). 


\begin{tabular}{|c|c|c|}
\hline radio & \multicolumn{2}{|c|}{ orientation } \\
\hline properties & face-on & edge-on \\
\hline Radio quiet & $\begin{array}{l}\text { Seyfert } 1 \\
\text { radio-quiet quasars }\end{array}$ & Seyfert 2 \\
\hline Radio loud & $\begin{array}{l}\text { BL Lac } \\
\text { BLRG } \\
\text { quasars }\end{array}$ & NLRG \\
\hline
\end{tabular}

Table 1.1: Unification model of AGN.

\subsubsection{The standard model.}

The main idea of this model is that all types of AGN correspond to the same physical phenomenon, e.g. infall of material onto a $\mathrm{SMBH}$, and that the observed differences are due to an orientation effect. Seyfert 1 and Seyfert 2 galaxies are then the same objects, viewed from different angles. Nuclei and accretion disks of type 1 Seyfert galaxies are seen face-on, while the central engine of Seyfert 2 galaxies is obscured by a dusty torus which prevents us to observe directly the broad emission lines and attenuate the AGN continuum. Seyfert 2 galaxies being three times more numerous than Seyfert 1 galaxies, the dusty torus must obscure about $3 / 4$ of the central source, which is in good agreement with the opening angle of the observed ionising cones (corresponding to the NLR). One strong observational evidence of this model, is the detection of weak broad emission lines in the polarisation spectrum of the Seyfert 2 galaxy NGC 1068. This polarised light is the AGN continuum and emission from the BLR, scattered by the ISM above the obscuring torus. Fig. 1.7 illustrates this simple unification scheme of Seyfert galaxies, showing the orientation effect on the observation of the different emitting regions of an AGN. We must however note that the orientation of AGN central regions (dusty torus and accretion disk) are unrelated to the orientation of the host galaxy (Nagar \& Wilson, 1999; Kinney et al., 2000). For example, the almost face-on galaxy M51 (disk inclination of about 20 ${ }^{\circ}$ ) hosts a Seyfert 2 active nucleus.

This unification can be extended to radio-loud AGN (Urry \& Padovani, 1995), the BLRG and NLRG being the radio-loud counterparts to the Seyfert 1 and Seyfert 2 galaxies, respectively. In a same way, BL Lac objects are seen face-on, directly along the radio jet which dominates all the spectrum, we then observed a featureless spectrum for these particular objects. Finally radio-quiet quasars are also seen face-on with a direct view on the continuum source. Table 1.1 summarises the aspects of this unification model.

Although this unification scheme explain well the different radio quiet objects in one hand and the radio loud object in the other hand, simple orientation considerations cannot explain why some objects are radio-loud while others are radio-quiet. The dichotomy between radio-loud and radio-quiet AGN is not yet well understood. The spin of the central SMBH may play a role in the radio-loudness of active galactic nuclei (Wilson \& Colbert, 1995; Sikora et al., 2007) 


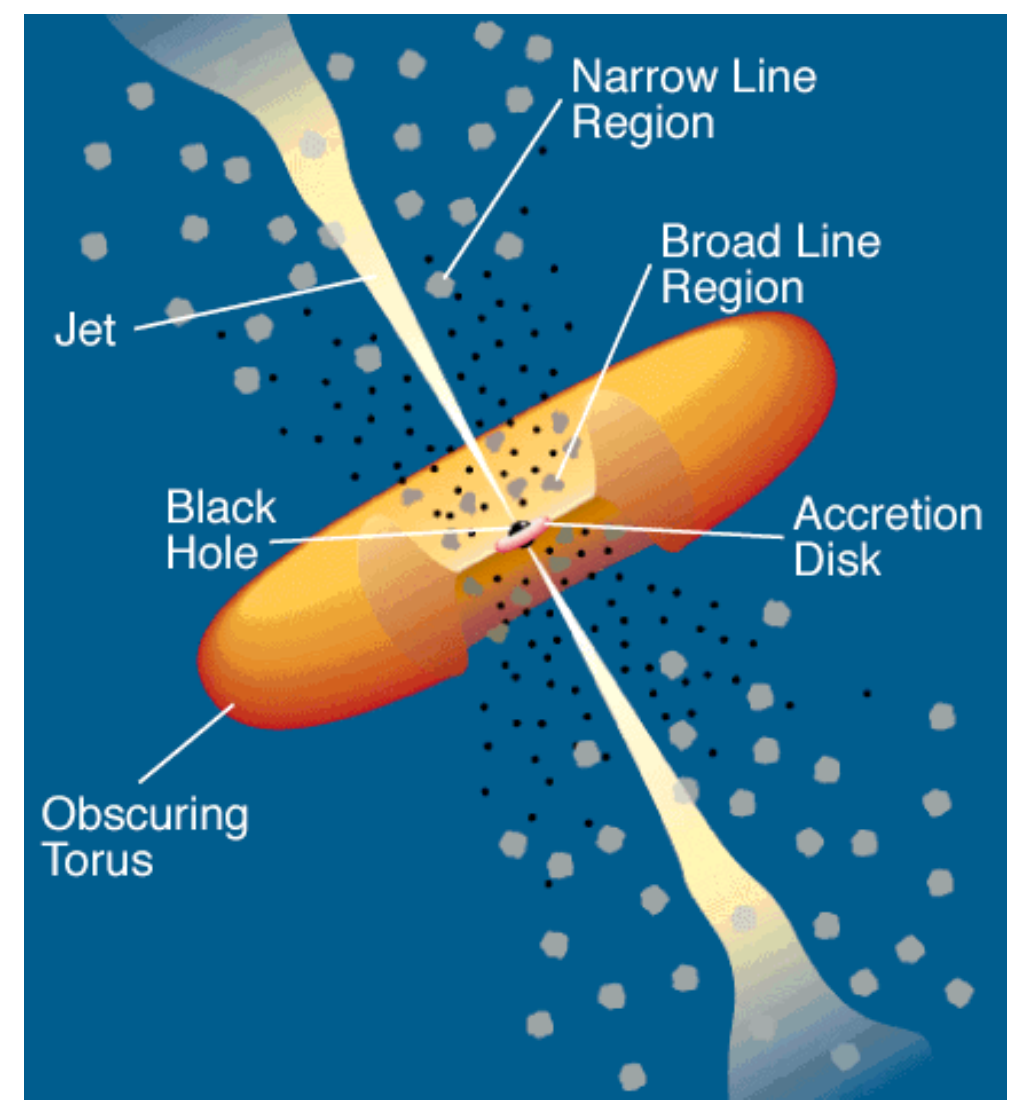

Figure 1.7: Unification scheme for the type 1 and 2 Seyfert galaxies (Urry \& Padovani, 1995). Spatial scales are not respected.

No direct observational evidence were available to support this observational scheme, due to the small size of the obscuring torus and accretion disk. However, with increasing spatial resolution instruments (e.g. HST, VLBI, IR interferometers) it is now possible to resolve structures on subarcseconds and submilliarcsecond scales. Hence, the dusty torus obscuring the active nucleus has been recently detected in the nearby Seyfert 2 NGC 1068 (Jaffe et al., 2004). This structure has a diameter of 3.4 pc. A larger similar dusty nuclear disk (125 pc in diameter) has also been observed in the radio-galaxies NGC 4261 (Jaffe et al., 1996). This dusty disk could also be assimilated to the torus obscuring the accretion disk and SMBH.

To give an idea of a possible structure of the AGN, Fig. 1.8 shows the probable locations of the different components of an AGN, from the closest to the $\mathrm{SMBH}$, the accretion disk, to the NLR at the kpc scale. 


\subsubsection{Alternative models.}

The standard unification model, with a SMBH surrounded by an accretion disk and a dusty obscuring torus is now widely accepted. However, some characteristics of AGN continuum spectra, and the fact that AGN have high luminosities on a wide range of wavelengths are not explained via this simple model.

An alternative model to the accretion onto a $\mathrm{SMBH}$ is the starburst model for low luminosity AGN (Terlevich et al., 1992). In this scenario the primary source of energy is coming from the numerous supernovae located in a central stellar cluster. However, this model cannot account for the the X-ray fluctuations and the radio continuum emission. 


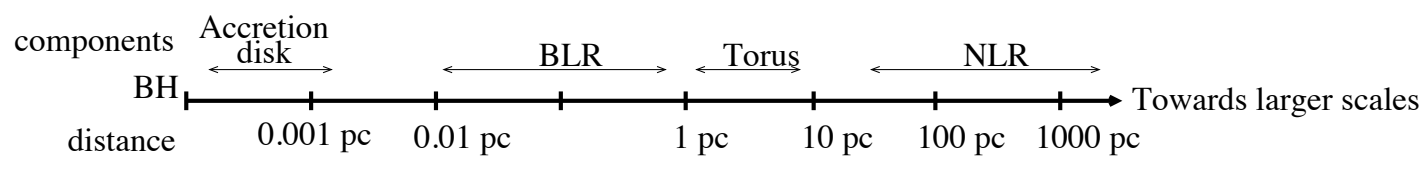

Figure 1.8: AGN components and spatial scales for a SMBH of $10^{7} \mathrm{M}_{\odot}$. The distances from the central black hole are displayed on a log scale. 


\section{Chapter 2}

\section{AGN-galaxy host connection. The fuelling issues.}

In the previous chapter, properties, similarities and differences of the different types of AGN have been reviewed. One particularity shared by all AGN is that they live in the centre of galaxies. It is then natural to wonder what are the effects of the host galaxy on the AGN and vice-versa. While it is now widely accepted that AGN are powered by accretion of gas onto a central SMBH (see Sec. 1.4), the mechanisms that remove angular momentum and transport gas towards the nucleus are not known. In particular, the role of the host galaxy and its environment in such processes remain unsolved questions. In this chapter, I focus on the AGN/galaxy host connection, and on the problem of fuelling low luminosity AGN in the local Universe, which is at the heart of this thesis work.

\subsection{Evolution of AGN}

AGN being very luminous objects, they can be seen at high redshift, and indeed quasars are found up to $z=6$ (the currently highest redshift quasar lies at $z=6.42$, Fan et al., 2003). It is therefore possible to study the distribution of quasars as a function of $z$ and thus the cosmic evolution of the population of this class of AGN. The luminosity function of QSOs at different redshifts (Fig. 2.1) shows a strong cosmic evolution. The space density of quasars as a function of redshift can then be determined as shown on Fig. 2.2 (Combes, 2006). This distribution presents a peak between redshift 2 and 3 (the quasars epoch), and declines steeply for more recent times. This shape is observed both in radio, optical or X-rays surveys. This implies that the AGN phenomenon was much more frequent at $z \simeq 2-3$ than at the present time.

Moreover, it is recognised that all bulge-dominated local galaxies host a $\mathrm{SMBH}$, and the mass of this black hole is correlated with the bulge luminosity and stellar velocity dispersion (Ferrarese \& Merritt, 2000; Gebhardt et al., 2000; 
Tremaine et al., 2002):

$$
M_{B H} \simeq 1.510^{8} M_{\odot}\left(\frac{\sigma_{*}}{200 k m s^{-1}}\right)^{\alpha}
$$

with a slope $\alpha \sim 4$. This relation has been reported in quiescent and active galaxies with black hole masses between $10^{6}$ and $10^{10} \mathrm{M}_{\odot}$ and it seems to hold for bright QSOs up to $z \sim 3$ (Shields et al., 2003). However, recent results reveal a cosmic evolution of the $M_{B H}-\sigma_{*}$ relationship (Treu et al., 2004; Salviander et al., 2007).

Recently, Ferrarese et al. (2006) constrained the ratio between their so-called Central Massive Object (CMO, either a $\mathrm{SMBH}$ or a compact stellar nucleus) and the galaxy masses: $\frac{M_{C M O}}{M_{\text {gal }}} \approx 0.18 \%$.

Such correlations between the central engine properties and the host galaxy properties (at larger scales) imply that the evolution of SMBH and their host galaxies are intimately related and that most today galaxies harbour dead quasars. All galaxies must have therefore experienced at least an accretion phase to form the central SMBH. Mean lifetime of nuclear activity can be estimated from the size and expansion rates of the radio lobes of radio-galaxies and it is of the order of $\sim 10^{8} \mathrm{yr}$. Successive radio-lobes along the jet axis provide direct evidence for the successive activity phases. Nuclear activity is then a relatively short-term event, possibly recurrent and most of the galaxies are active during the quasars epoch. The drop-off in QSO density for $z<2$ can then be explained by a decrease of accretion rates onto the $\mathrm{SMBH}$, either because of a lack of fuel or of feedback processes that prevent material to be funnelled inwards. Given that central SMBH are ubiquitous in today's galaxies and that a small fraction of these present-day galaxies shows signs of ongoing activity (Seyfert galaxies being found in 3 percent of local galaxies, Kewley et al., 2006), re-ignition of quiescent $\mathrm{SMBH}$ is required. One key question is whether the triggering and fuelling mechanisms are related to the host galaxy properties.

\subsection{Fuelling the AGN}

To fully understand the fuelling of AGN, one has to constrain the origin of the fuel and the mechanisms which transport this fuel towards the nucleus. In order to be accreted onto the central SMBH, material must lose nearly all its angular momentum. The angular momentum per unit of mass is $L=(G M R)^{1 / 2}$, where $R$ is the radius of the considered orbit, $\mathrm{M}$ is the mass enclosed within $R$ and $G$ the gravitational constant. Therefore, to drive fuel from the outskirts of the galaxies $(R \simeq 10 \mathrm{kpc}$ ) within the central regions $(R \simeq 0.01 \mathrm{pc})$ onto a SMBH of $10^{7} \mathrm{M}_{\odot}$, the angular momentum must decrease by $\sim 10^{-6}$ of its initial value. The nature of the physical processes able to remove such amount of angular momentum is then one of the most important issues in the context of AGN fuelling. 


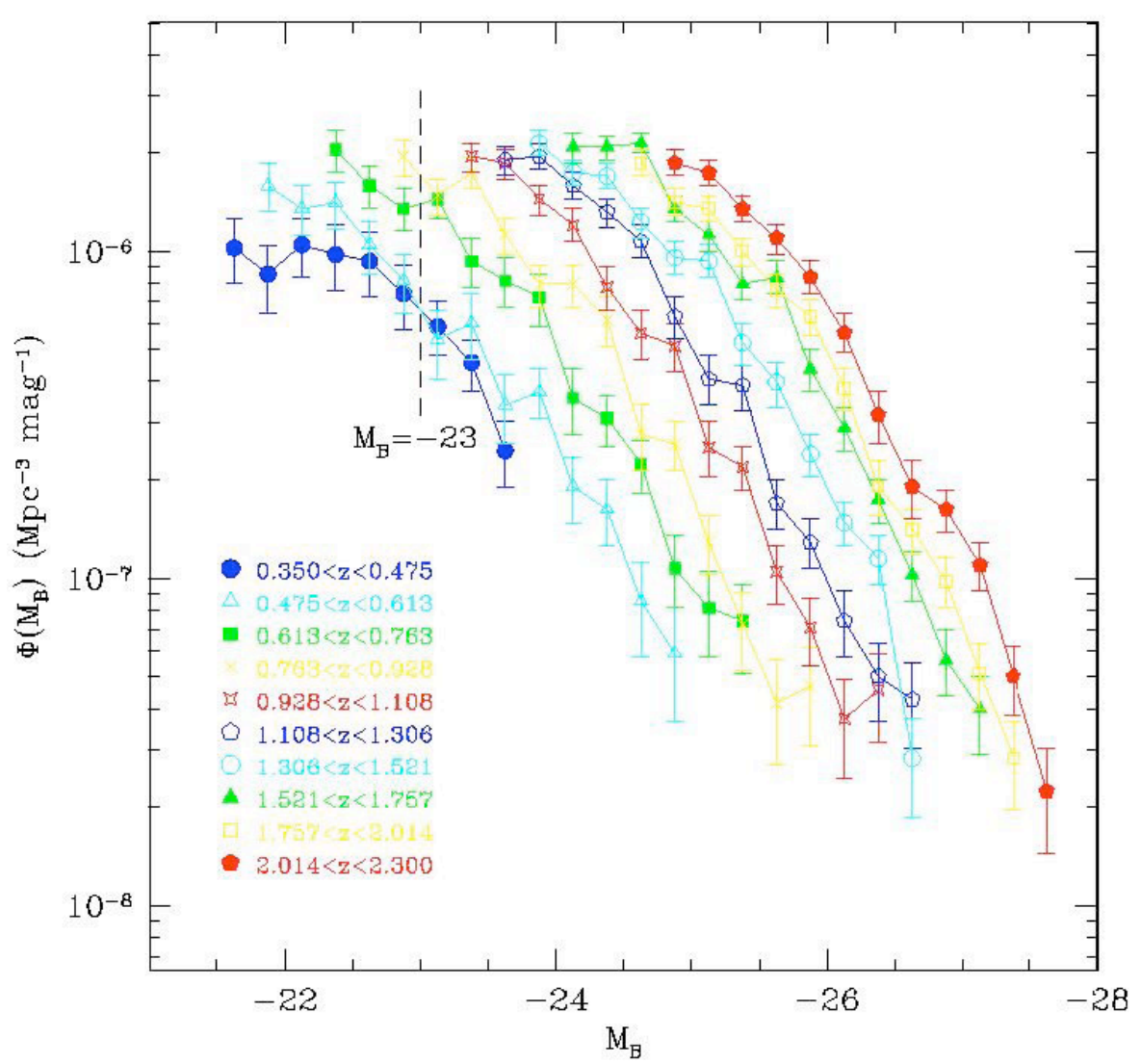

Figure 2.1: QSO luminosity function at redshift $0.35 \lesssim z \lesssim 2.3$ from Boyle et al. (2000).

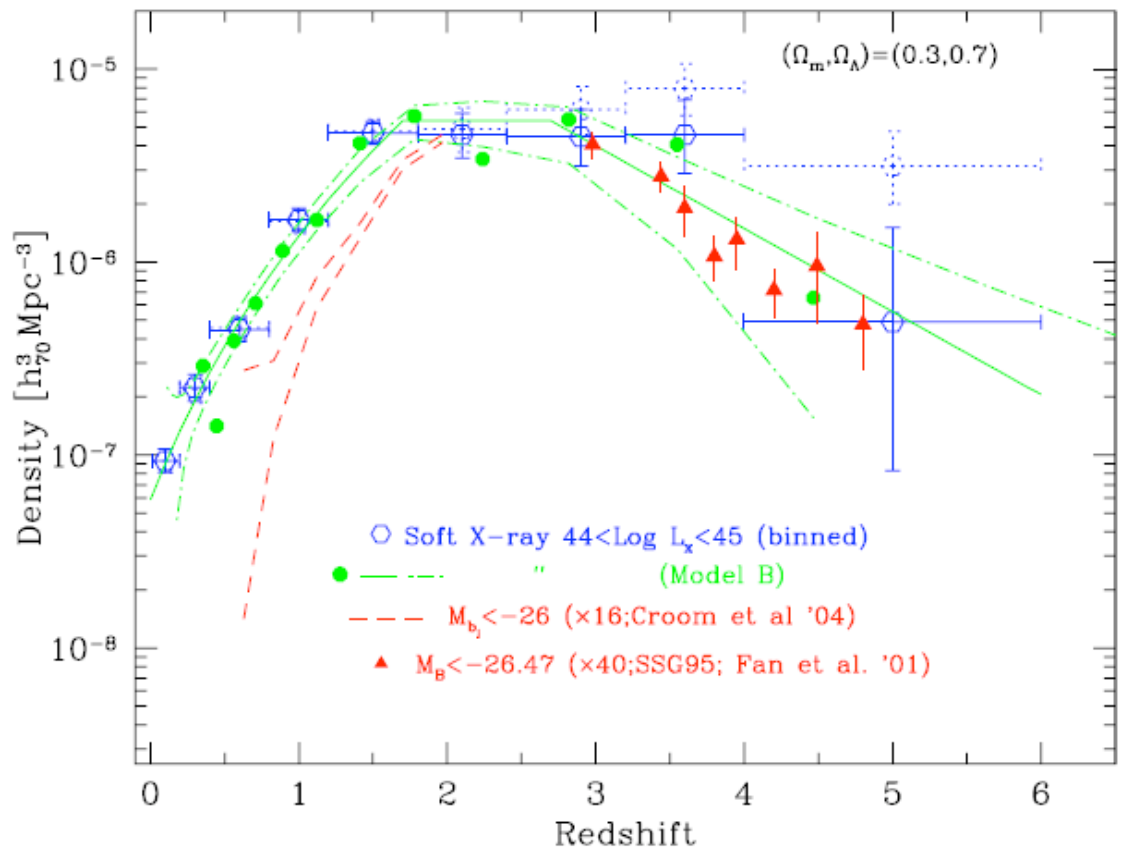

Figure 2.2: Distribution of the quasars space density as a function of redshift (Combes, 2006). Data are from Hasinger (2005): soft X-ray selected (empty blue circles) and optically selected sources (dashed lines and red filled triangles); Croom et al. (2004): optically selected QSO (dashed red curves). 
Several mechanisms have been proposed to transfer angular momentum efficiently and to provide material close to the $\mathrm{SMBH}$, feeding then the nuclear activity. Shlosman et al. (1990); Combes (2001) and more recently Wada (2004) reviewed these different mechanisms. Given the wide range of spatial scales involved in fuelling the AGN, it is very likely that several of these mechanisms take place in different regions of the galaxy. Gravitational perturbations due to external (tidal interaction, galaxy mergers) or internal (stellar bar, spiral arms, warps) instabilities and hydrodynamic mechanisms (turbulence in the ISM, viscosity in accretion disks) are potentially good drivers to transport material towards nuclear regions. Extensive numerical and observational studies have been done to investigate the role and importance of the different transportation mechanisms on the nuclear activity. In this section, I review the different results obtained by these previous studies.

\subsubsection{Galaxy interactions and mergers}

Mergers and galactic encounters may lead to strong gravitational perturbations in the galactic disk, which can trigger the infall of gas in the nuclear regions. Numerical simulations predict large gas radial inflows due to mergers of galaxies (Barnes \& Hernquist, 1991; Mihos \& Hernquist, 1996; Hernquist \& Mihos, 1995), down to the inner 10 pc of the galaxy. A strong correlation between nuclear activity and galaxy interactions exists for the very luminous AGN, such as quasars, QSOs or FR-II radio-galaxies. High redshift quasars are predominantly found in interacting systems with evidence of tidal interactions (Hutchings \& Neff, 1988; Bahcall et al., 1995). Radio-loud quasars present about 4 times more companions and radio-quiet quasars 2 times more than non-active galaxies (Kirhakos et al., 1999). Similarly, FR-II radio-galaxies seem to be in high density environment, where many galaxy interactions occur (Yates et al., 1989). Galaxy interactions and mergers seem to be the predominant mechanisms which trigger and then sustain nuclear activity in these high luminosity objects.

However, this scenario is unlikely for local low-luminosity AGN such as Seyfert galaxies. While some imaging studies found an excess of companions for Seyfert galaxies relative to non-active galaxies (Laurikainen \& Salo, 1995; Rafanelli et al., 1995), others claimed that environment of Seyfert and non-active galaxies is the same (De Robertis et al., 1998). As investigated by Osterbrock (1993) and Heckman (1990), such studies are very sensitive to the sample selection which could explain the different results. In order to avoid selection biases, Schmitt (2001) selected Seyfert and non-active control galaxies samples with matching host properties, from the Palomar survey (Ho et al., 1997). Comparing the local environment of galaxies of different types of activity, they found that there is no statistically-significant excess of companions for Seyfert galaxies as compared with non-active galaxies (including $\mathrm{H}$ II and LINER galaxies), taking into account the relation between morphological type and the local galaxies density. Ho et al. (2003) reached exactly the same con- 
clusion with the same sample. This result was confirmed on larger scales by Sorrentino et al. (2006) who found no evidence of relation between large-scale environment properties and the nuclear activity. The difference of frequency of companions between Seyfert and non-active galaxies found in earlier studies is due to a morphology-density effect.

In the case of low-luminosity AGN, interaction of galaxies is then not the mechanism which fuel the SMBH. Other processes of transportation of gas on scales $\lesssim 0.1-1$ pc and actually feeds the black hole reservoir, sustaining the AGN through its lifetime, are therefore required.

\subsubsection{Internal gravitational instabilities}

Triaxial gravitational potentials, such as in the presence of a stellar tumbling bar, spirals waves or lopsidedness, lead to the presence of gaseous radial streaming. Different families of periodic stellar orbits exist in a barred potential (Contopoulos \& Papayannopoulos, 1980). Among them, the $x_{1}$ family, oriented along the bar major-axis, is the main family supporting the bar. $x_{1}$ orbits extend between the centre and the corotation resonance (CR) of the bar if no inner Lindblad resonance (ILR) is present. If there is an ILR, another family of orbits appears between the centre and the ILR. These $x_{2}$ orbits are perpendicular to the bar major-axis. The $x_{1}$ orbits extend then from the ILR to the CR. When two ILR are present, $x_{2}$ orbits take place between the inner ILR (IILR) and the outer ILR (OILR).

In a tumbling bar potential, the orientations of the periodic orbits are expected to rotate by $90^{\circ}$ at each resonance. The gas should experience shocks in the regions where the orbits do cross, due to its collisional and dissipative natures. Beyond the CR, gas is driven towards the outer Lindblad resonance (OLR), while between the CR and the ILR (or OILR) the gas loses its angular momentum. Hydrodynamical simulations of gas in a barred potential have shown indeed that gas is redistributed, sometimes producing regions of shocks, and can fall towards the nuclear region and settle in an inner ring at scales of about 1 kpc (Athanassoula, 1992; Friedli \& Benz, 1993; Piner et al., 1995). Many observational studies provided evidence of such gaseous streaming in a barred potential (e.g. Vila-Vilaro et al., 1995; Koribalski et al., 1996; Mundell et al., 1999; Jogee et al., 2005).

However, a single large-scale bar cannot transport gas below a few hundreds parsecs where the gas accumulates after crossing the ILR. Another mechanism has to take over to fuel material closer to the nucleus. A scenario of bars within bars has been developed (Shlosman et al., 1989; Maciejewski \& Sparke, 1997; Erwin \& Sparke, 2002) to model the fuelling of the nucleus. The gas is transported via a large-scale bar towards the centre creating a gas reservoir in the central kpc region. Then a stellar nuclear bar, embedded within the large-scale bar, forms via gravitational instabilities and allows to transport the gas from the kpc-scale reservoir down to about $10 \mathrm{pc}$. Imaging studies revealed nested nuclear bars in about a third of early-type barred galaxies (Laine et al., 2002; Erwin \& Sparke, 2002). Spiral instabilities are also efficient to create gaseous 
streaming, via shocks. Recently, observational studies showed that nuclear dust and gaseous spirals are common in nearby galaxies (Regan \& Mulchaey, 1999; Martini \& Pogge, 1999; Laine et al., 2001) and these nuclear spirals have been evoked to explain the transportation of material from the kpc-scale down to the inner 10 pc regions (Englmaier \& Shlosman, 2001; Maciejewski et al., 2002; Maciejewski, 2004a,b).

Given that large-scale bars, nuclear bars and nuclear spirals are commonly found in nearby galaxies, and that they seem to be efficient drivers to transport gas inwards, many imaging studies investigated the role of such structures in nuclear activity. In particular recent high resolution NIR or optical imaging observations have been carried on samples of Seyfert and properly matched non-active galaxies (e.g. morphological type, distance, total luminosity), in order to compare the fraction of bars in these two samples. In term of largescale bars, these studies revealed either no significant correlation between the presence of large-scale stellar bars and nuclear activity (Mulchaey \& Regan, 1997; Martini et al., 2003) or an excess of bars in active galaxies relatively to non-active galaxies (Knapen et al., 2000; Laine et al., 2002; Laurikainen et al., 2004a), depending on the samples definition and the methods used to recognise bars in the data.

Thus, whether Seyfert galaxies present an excess of bars compared to nonactive galaxies remains an open question. However, if any distinction between Seyfert and non-active galaxies regarding the frequency of bars exists, it should be small. Similar studies investigated the presence of secondary bars in nearby galaxies. They report that nuclear bars are not found preferentially in Seyfert galaxies, compared to non-active galaxies (Regan \& Mulchaey, 1999; Erwin \& Sparke, 2002; Laine et al., 2002).

In a similar context, it has been found that dust or gaseous nuclear spirals occur with comparable frequency in active and non-active galaxies as shown by Martini et al. (2003). However, they also found that all the AGN of their sample possess dust structure in the circumnuclear regions, while a fraction of the non-active galaxies (25\%) display no dust structure at all. More recently, Hunt \& Malkan (2004) and Simões Lopes et al. (2007) investigated the relation between the circumnuclear dust and gaseous structure and the nuclear activity.

Hunt \& Malkan (2004) conducted a NIR NICMOS/HST imaging study of the circumnuclear regions of 250 nearby galaxies. Their sample comprises nonactive, H II / starburst, LINER and Seyfert galaxies, with similar host galaxies properties and similar parsec-to-pixel spatial scales. They investigated quantitatively the nuclear morphology of these galaxies in terms of asymmetric structures (e.g. isophotal twists, bars, boxy/disky isophotes) and compared the results of the morphology analysis, in the context of the AGN fuelling. Their most robust result is the significant excess of circumnuclear isophotal twists in Seyfert 2 galaxies relative to non-active galaxies and the other active galaxies, in particular Seyfert 1's. The structures responsible for such morphological features may cause also kinematic perturbations at these spatial scales. Their results suggest the presence of identifiable kinematic differences between Seyfert 
and inactive galaxies in the central kpc regions.

Similarly, Simões Lopes et al. (2007) investigated the circumnuclear dust morphology of a well-matched Seyfert and non-active galaxies sample, using structure maps (Pogge \& Martini, 2002). All their active galaxies possess circumnuclear dust, while dust is present in only $27 \%$ of the early-type inactive galaxies. In contrast, $38 \%$ of early-type non-active galaxies present nuclear stellar disks, while none has been detected in active early-type galaxies. Their results provide a clear connection between dust and nuclear activity in earlytypes galaxies. However they could not constrain the origin of the circumnuclear dust or the nature of the fuelling mechanisms. They suggest that investigation of the kinematics of dust, with improved models of dust settling and destruction in the circumnuclear regions, could reveal the origin of the dust and the fuelling mechanisms for these early-type AGN.

Fuelling and growth of SMBH in the most distant and luminous AGN is then associated with the violent dynamical processes related to galaxy interactions and mergers, during the galaxy formation and early evolution. In the more nearby Universe, gravitational instabilities such as stellar bars or spirals are efficient potential actors to transport material inwards. However, these mechanisms do not seem to be at work in the actual SMBH fuelling of the nearby low-luminosity AGN such as Seyfert galaxies: no conclusive distinction has been found so far between Seyfert nuclei hosts and non-active galaxy morphologies on a range of spatial scales that encompasses nearby companions / galactic interactions, large-scale bars and nuclear spirals. Recent IR imaging studies (Hunt \& Malkan, 2004; Simões Lopes et al., 2007) suggest the possibility of kinematic differences between nearby active and non-active host galaxies. Investigating the kinematics of Seyfert galaxies at these spatial scales to probe the dynamical structures may reveal the underlying processes responsible for the transport of material from kpc scales to pc scales.

\subsection{Two-dimensional kinematics of Seyfert galax- ies}

As explained above, imaging studies of AGN alone cannot constrain the fuelling mechanisms of the nuclear activity. Probing the dynamics of Seyfert galaxies requires spectroscopic data. Single aperture and long-slit spectroscopy studies are clearly inadequate to investigate the complex structures observed in the central kpc of Seyfert galaxies. Two-dimensional spectroscopy (integral-field spectroscopy, IFS hereafter) is therefore a pre-requisite to study the dynamics of the gaseous and stellar components. 


\section{TIGER MODE OPTICAL LAYOUT}

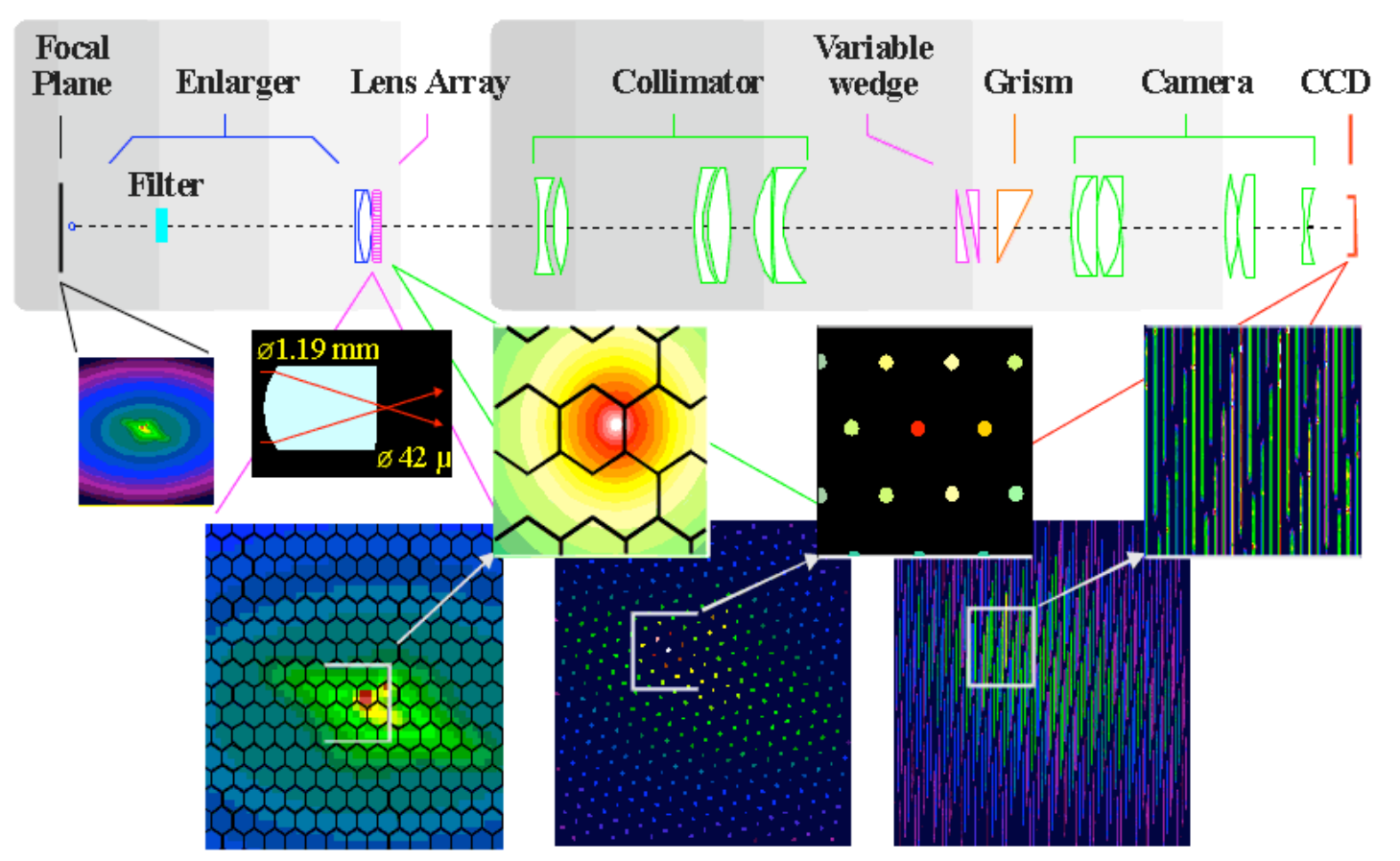

Figure 2.3: OASIS optical layout. The main optical elements are displayed from the telescope focal plane on the left to the CCD plane on the right. An image of a galaxy is shown at different stages in the instrument; from left to right: the telescope focal plane, the entrance plane of the lens array, the exit plane of the lens array and the detector plane. The light path inside a microlens is also shown. 


\subsubsection{Integral-Field Spectroscopy data}

In order to have two-dimensional kinematic information of galactic disks, optical and IR integral-field spectrographs where developed. Several types of integral-field spectrographs exist: Fabry-Perot spectrographs which allow to map a large field of view (FOV), but in a very narrow wavelengths band; multifibres systems or array of microlenses were used to divide the FOV in numerous spatial pixels (spaxels) on which spectra are derived. Spectrographs such as INTEGRAL use a multifibres system while OASIS or SAURON are formed of a microlenses array. Fig. 2.3 shows the optical layout of the OASIS integralfield units (IFU). SAURON layout is based on the same scheme, with squared lenses. After selecting the wavelength window with a filter, the object is imaged on the lenslet array. Each microlens of this array produces a micropupil which is dispersed by a grism. The grism is slightly rotated with respect to the lenslet array to avoid overlap of adjacent spectra. A camera then images the resulting spectra on a CCD aligned with the dispersion direction.

Of course, radio interferometers (e.g. VLA, Plateau de Bure Interferometer) allow also to map the two-dimensional kinematics of galaxies in the radio wavelengths.

Early work with such instruments provided two-dimensional gas and stellar kinematic maps of the central parts of a few nearby active galaxies: e.g. NGC 4151 (Mediavilla \& Arribas, 1995), NGC 3227 (Mediavilla \& Arribas, 1993), NGC 1068 (Garcia-Lorenzo et al., 1997) with INTEGRAL, or NGC 2110 (Ferruit et al., 2004) using OASIS. Multiple gaseous systems and kinematic perturbations are sometimes revealed, but the corresponding field-of-view (FOV) and/or the angular resolution were often too small to disentangle the AGN-related and galactic disc line emissions. Statistical conclusions cannot be drawn from these detailed studies of individual Seyfert galaxies, originally targeted for their complex nuclear properties and lacking any control inactive galaxy comparison. More recently, the molecular gas in the central part of a larger (though still small) sample of Seyfert galaxies was observed using the Plateau de Bure Interferometer in the course of the NUGA survey (GarcíaBurillo et al., 2003), revealing the potential role of gravity torques in the feeding of the inner 100 pc (e.g. García-Burillo et al., 2005). Gas responds non-linearly to deviations from axisymmetry so a more direct link with the gravitational potential is still difficult to establish, due to the lack of large-scale stellar kinematic maps for these galaxies. Six nearby Seyferts were also observed with GMOS/ Gemini, enabling the mapping of the ionised gas and stellar kinematics in the central few arcseconds (Barbosa et al., 2006). Integral-field spectrographs such as SINFONI/VLT reached regions even closer to the nucleus (Davies et al., 2006), thanks to the use of adaptive optics. Again, such observations are useful for studying the kinematics in the nuclear regions, identifying the putative molecular torus around the $\mathrm{SMBH}$, retrieving the characteristics of the central engine such as the mass of the $\mathrm{SMBH}$, or studying the environment of the active nucleus. However, observed kinematics at these relatively small scales are rather difficult to interpret in the context of the host galaxy, 
considering the significant influence of non-gravitationally driven processes (e.g. outflows).

\subsubsection{Motivation of this thesis}

In this thesis, I focused on low luminosity, radio-quiet nearby active galaxies, namely the Seyfert galaxies. The gas and stellar kinematics of these galaxies can be resolved on spatial scales down to a few parsecs. Investigation of gas flow in central few hundreds of parsec is then possible. As discussed in Sec. 2.3.1, IFS data are required to investigate in detail the dynamics in the central regions of Seyfert galaxies. Such data allow to investigate the complex structure and kinematics of ionised gas and stars, on arcsec scales and to reveal potential streaming signatures. However, integral-field spectrographs do not have a very large FOV, e.g. the SAURON IFU FOV corresponds to $33^{\prime \prime} \times 41^{\prime \prime}$, we do not then have access to kinematic information on large spatial scales, corresponding to the dynamics of e.g. the outer disk of the host galaxy itself. Therefore, in order to constrain the fuelling mechanisms in Seyfert galaxies, from the outskirts of the galaxies $(\sim 10 \mathrm{kpc})$ down to the circumnuclear regions ( $\sim 10 \mathrm{pc}$ ) and investigate the connection between the host galaxy and the AGN, kinematic data on larger scale are required in addition to IFS data in the circumnuclear regions. Neutral hydrogen $(\mathrm{HI})$ is often the most spatially extended component of the galactic disk. It is then very sensitive to galaxy interactions and mergers and is also a potential tracer of non-axisymmetric gravitational potential (e.g. bar, spiral arms). H I synthesis imaging thus provides unique information on the global mass distribution and dynamics of the galactic disk at large radii and a long-term dynamical history of the galaxy via kinematic features. Current $\mathrm{H}$ I imaging interferometers however cannot routinely image diffuse gas on scales smaller than $\sim 15^{\prime \prime}$.

Then, optical IFS and H I observations are complementary and allow to map the kinematics and morphology of the large-scale gas disk properties as well as the circumnuclear regions.

A project was therefore designed to conduct a comprehensive study of the neutral gas, ionised gas and stellar kinematics of nearby active and inactive galaxies using the VLA and the SAURON IFU on the WHT. Consequently, an international collaboration, including Eric Emsellem, Pierre Ferruit and myself at CRAL - Observatoire de Lyon (France), Carole Mundell at ARI, Liverpool (UK) and Neil Nagar in the Astronomy Group, Concepciòn (Chile) initiated the first statistically-significant investigation of the circumnuclear regions and outer disks of galaxies in a well-defined distance-limited sample $\left(\mathrm{V}_{\text {sys }}<4000\right.$ $\mathrm{km} \mathrm{s}^{-1}$ ): namely, 28 Seyfert galaxies selected from the RSA catalogue, paired with 28 control inactive galaxies with carefully matched optical properties (Mundell et al., 2007). This project aims to compare the morphological and kinematic properties of active and inactive galaxies. We conducted the first multi-wavelength observation campaign to investigate the host galaxy struc- 
ture and kinematics on spatial scales from the outer disk to the inner regions via two observational surveys:

1. The VHIKINGS survey (VLA Hydrogen Imaging and Kinematics of INactive Galaxies and Seyferts survey) aims at quantifying the properties of the atomic gas in the Seyfert and inactive galaxies of the master sample.

2. The SAURON/Seyfert survey extends the study of structures and kinematics to the inner $1 \mathrm{kpc}$ of a subsample of galaxies selected from the VHIKINGS sample. In particular, the galactic potential of the host galaxy is probed by comparing the properties of both the ionised gas and stellar components in the circumnuclear regions of Seyfert and inactive galaxies, with the ultimate aim of connecting the large-scale properties traced by the neutral hydrogen with the circumnuclear regions. The SAURON IFS, mounted on the William Herschel Telescope (La Palma, Spain) has a large enough FOV to observe ionised gas under the influence of the galaxy host potential, and a high enough spatial sampling to still probe the AGNrelated emission in the inner parts. These data are then well suited to study both the stellar and gaseous kinematics in the above-mentioned context of fuelling. A number of key studies were recently performed with SAURON providing unique datasets for a sample of nearby earlytype (de Zeeuw et al., 2002; Emsellem et al., 2004; Sarzi et al., 2006) and spiral galaxies (Ganda et al., 2006; Falcón-Barroso et al., 2006). As a spin off, the distribution and kinematics of the gas and stellar components were also obtained and studied for some well known Seyfert (NGC 1068, Emsellem et al., 2006, NGC 5448, Fathi et al., 2005) or spiral inactive galaxies like M 100 (Allard et al., 2006). These works provided evidence for the existence of gaseous inward streaming in the inner few $\mathrm{kpc}$, hinting at a mechanism for transporting gas in the circumnuclear regions and further.

In the following, Chapter 3 describes the sample selection, the observations and the data reduction done during this work. The moments maps of our optical and radio data are presented in Chapter 4 and kinematic analyses of the velocity fields are discussed in Chapter 5. Then in Chapter 6, I discuss the results of this work in the context of fuelling the AGN and present the perspectives of this study. 


\section{Chapter 3}

\section{Sample selection, observations and data reduction}

In Chapter 2, we discussed the need of a multi-wavelength study of Seyfert and non-active galaxies to investigate the kinematic structures at all scales and interpret them in the context of AGN fuelling. Such study requires twodimensional kinematic data, and a well-defined sample of active and nonactive galaxies in order to perform a comparative analysis.

In this chapter, I first focus on the selection of the sample made of nearby Seyfert and non-active galaxies, with well-matched host galaxy properties. Then I describe the observations of these galaxies and the corresponding data reduction.

\subsection{Sample selection}

\subsubsection{The parent sample}

One important constraint in the comparative studies of active and non-active galaxies is to avoid any sample-selection bias (e.g. morphological type, spatial resolution, luminosity) which could invalidate the results. We selected galaxies from the Revised Shapley Ames (RSA) Catalogue of Bright Galaxies with complete nuclear spectroscopic classification provided by Ho et al. (1997). They listed the nuclear spectroscopy properties of almost 500 galaxies from the RSA catalogue, with a wide range of host galaxy properties such as Hubble type, absolute magnitude or disk inclination and provided a robust nuclear activity classification of these galaxies.

Our master sample comprises the 39 brightest Seyfert galaxies of the RSA catalogue, with B-band magnitude $\mathrm{B}_{T}<12.5 \mathrm{mag}$, systemic velocity $\mathrm{V}_{\text {sys }}<$ $4000 \mathrm{~km} \mathrm{~s}^{-1}$, and disk inclination $i$ between 20 and $70^{\circ}$. The Seyfert galaxies are paired with 39 control galaxies with carefully matched optical proper- 


\begin{tabular}{lll}
\hline Galaxy Properties & $\begin{array}{l}\text { Seyfert Selection } \\
\text { Criteria }\end{array}$ & $\begin{array}{l}\text { Control Galaxy } \\
\text { Selection Criteria }\end{array}$ \\
\hline B-band apparent magnitude & $\mathrm{B}_{T} \leq 12.5$ & $-0.45 \leq\left[\mathrm{B}_{T}(\mathrm{~S})-\mathrm{B}_{T}(\mathrm{C})\right] \leq+0.73$ \\
Systemic velocity $(\mathrm{km} / \mathrm{s})$ & $\mathrm{V}_{\text {sys }} \leq 4000$ & $-900 \leq\left[\mathrm{V}_{\text {sys }}(\mathrm{S})-\mathrm{V}_{\text {sys }}(\mathrm{C})\right] \leq 1900$ \\
Disc inclination (optical) & $20^{\circ} \leq i \leq 70^{\circ}$ & $-20^{\circ} \leq[i(\mathrm{~S})-i(\mathrm{C})] \leq+17^{\circ}$ \\
Declination limit & $\delta>20^{\circ}$ & \\
\hline
\end{tabular}

Table 3.1: Sample selection criteria for the Seyfert and their control galaxies. The constraints on the B-band magnitude, systemic velocity, disk inclination and declination limits are listed for the Seyfert galaxies and the control nonactive galaxies. .

ties, such as $\mathrm{B}_{T}, \mathrm{~V}_{\text {sys }} i$ and morphological type. For the latter, classification from RC3 was used and confirmed by eye using Digital Sky Survey (DSS) images of each galaxies. Table 3.1 lists the selection criteria used to define this sample of Seyfert and inactive galaxies. Seyfert and control galaxies are also closely matched in optical morphological type. Fig. 3.1 shows the Hubble type $\mathrm{T}$, disc inclination, B-band magnitude and $\mathrm{D}_{25}$ for each pair of Seyfert/nonactive galaxies. The active and non-active galaxies in the master sample and their properties are listed in Table A.1 (Appendix A). 

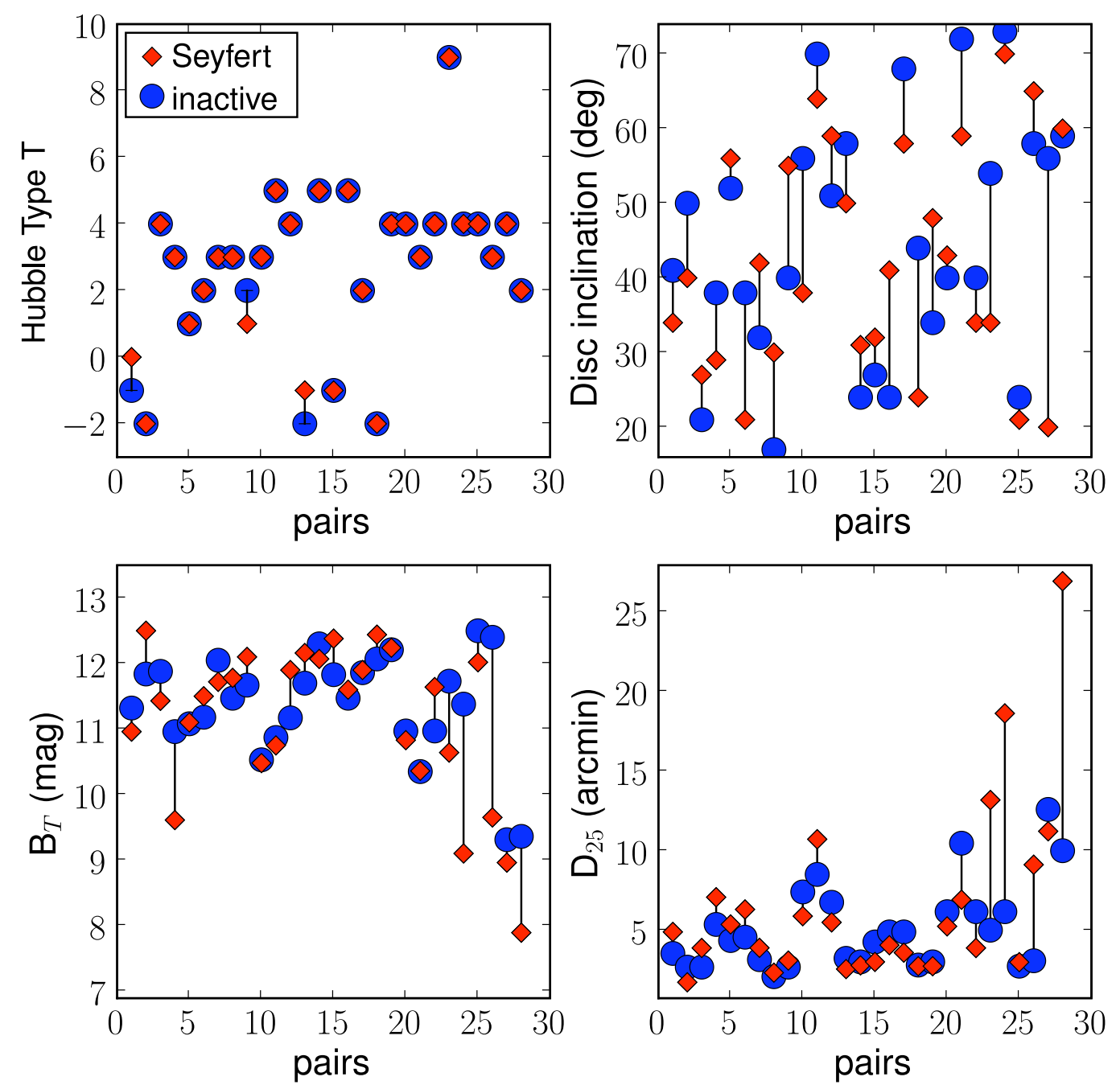

Figure 3.1: Properties of the master sample galaxies. Only the 28 brightest Seyfert galaxies of the master sample and their control galaxies are shown Red filled diamonds and blue filled circles correspond to the Seyfert and their associated non-active galaxies, respectively. In each plot, the galaxies are displayed in pairs Seyfert/control, on the $X$ axis. The top left plot presents the Hubble type of the Seyfert and non-active galaxies, the top right plot presents the disk inclination of each galaxy, the bottom left plot presents the B magnitude of the galaxies and the bottom right plot presents the $\mathrm{D}_{25}$ of the galaxies. 


\begin{tabular}{|l|c|c|c|c|}
\hline Nuclear Types & {$[\mathrm{O} \mathrm{III}] / \mathrm{H} \beta$} & {$[\mathrm{N}$ II $] / \mathrm{H} \alpha$} & {$[\mathrm{S} \mathrm{II}] / \mathrm{H} \alpha$} & {$[\mathrm{O} \mathrm{I}] / \mathrm{H} \alpha$} \\
\hline HII regions & Any & $<0.6$ & $<0.4$ & $<0.08$ \\
Seyfert nucleus & $>3$ & $\geqslant 0.6$ & $\geqslant 0.4$ & $\geqslant 0.08$ \\
LINERs & $<3$ & $\geqslant 0.6$ & $\geqslant 0.4$ & $\geqslant 0.17$ \\
Transitions objects & $<3$ & $\geqslant 0.6$ & $\geqslant 0.4$ & $\geqslant 0.08 ;<0.17$ \\
\hline
\end{tabular}

Table 3.2: Criteria for spectral classifications, taken from Ho et al. (1997).

\subsubsection{The nuclear classification}

As explained above, we used the nuclear spectroscopic classification of Ho et al. (1997) to distinguish between the different classes of nuclear activity: Seyfert, HII, LINER galaxies or transition objects. The transition objects are defined as galaxies with emission lines properties intermediate between LINER and H II nuclei. Table 3.2 lists the boundaries defined by Ho et al. (1997) between the different activity types. Our active galaxies sample is composed only of Seyfert galaxies, while H II, LINER galaxies and transition objects, considered as non-active galaxies, form the control sample. The spectral classification of each galaxy used throughout this study is listed in Column 12 of Table A.1.

Top row of Fig. 3.2 shows the $[\mathrm{O}$ III] $/ \mathrm{H} \beta$ versus [N II] $/ \mathrm{H} \alpha$, [O III] $/ \mathrm{H} \beta$ versus [S II] $/ \mathrm{H} \alpha$ and $[\mathrm{O} \mathrm{III}] / \mathrm{H} \beta$ versus [O I] $/ \mathrm{H} \alpha$ diagnostic diagrams for our sample, using lines ratio and limits between the different nuclear classes from Ho et al. (1997). A comparison with more recent classifications can be made. Bottom row of Fig. 3.2 presents the three same diagnostic diagrams (using the same line ratios) with recent criteria from Kewley et al. $(2001,2006)$ to distinguish between Seyfert, LINERs and normal galaxies. The Kewley et al. (2001) classification line (black line) provides an upper limit to the star-forming galaxies in the three diagrams. In the second and third diagrams, the Kewley et al. (2006) classification line (green line) separates the Seyfert galaxies from the LINERS and transitional objects.

As shown in these diagrams most of the nuclear classifications derived with simple criteria by Ho et al. (1997) and used in our study hold with the more recent separation criteria of Kewley et al. (2001) and Kewley et al. (2006). However, 16 objects (about $20 \%$ of our sample) are ambiguous: classified as one type of objects with the Ho et al. (1997) classifications and as another type with the criteria of Kewley et al. (2001, 2006). 

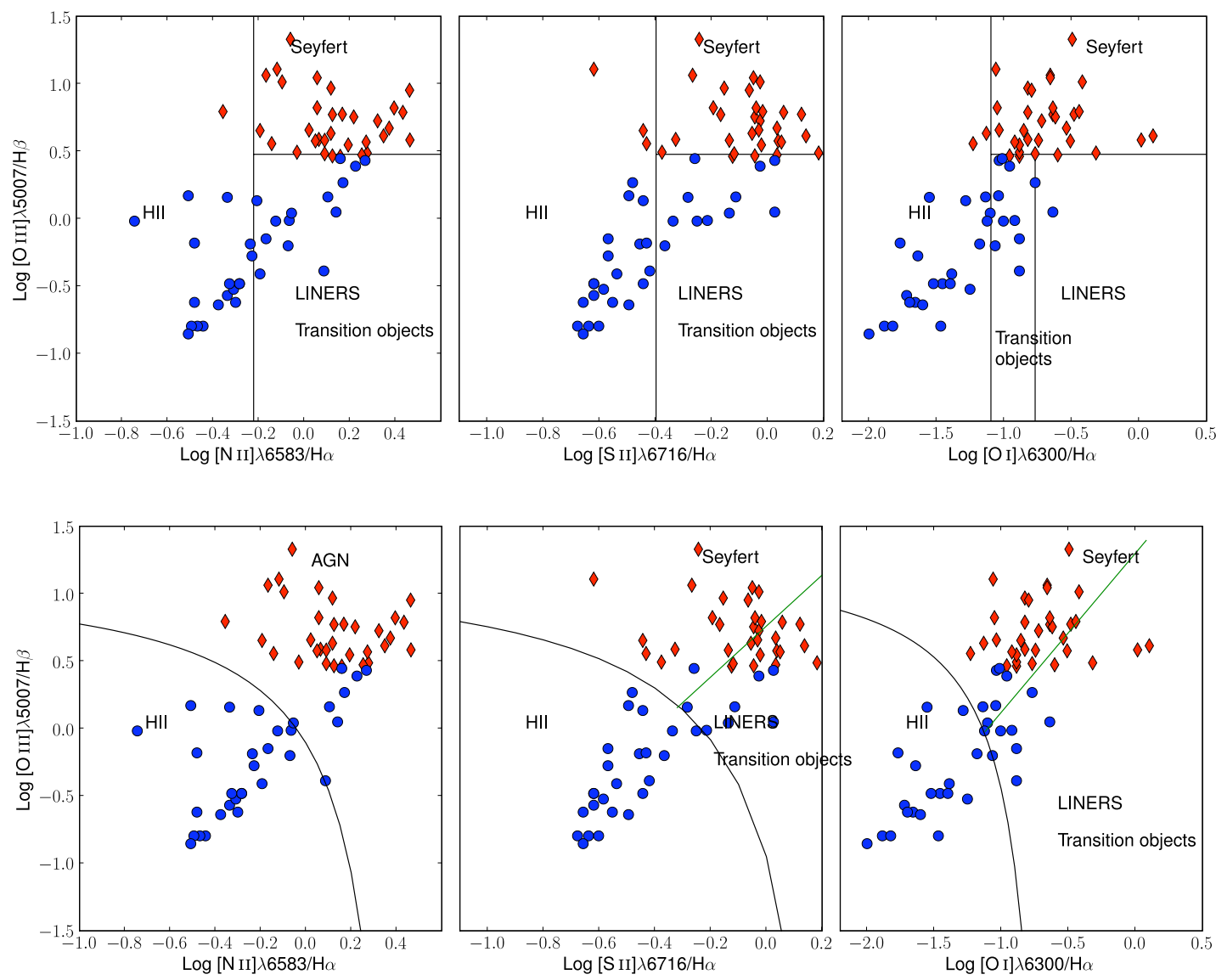

Figure 3.2: The [O III] $/ \mathrm{H} \beta$ versus [N II] $/ \mathrm{H} \alpha[\mathrm{O} \mathrm{III}] / \mathrm{H} \beta$ versus [S II] $/ \mathrm{H} \alpha$ and [O III $] / \mathrm{H} \beta$ versus $[\mathrm{O} \mathrm{I}] / \mathrm{H} \alpha$ diagnostic diagrams for our sample galaxies. The red diamonds represent the galaxies considered as Seyfert in our studies and the blue points correspond to their control non-active galaxies. In the top row, the boundaries from Ho et al. (1997) are used to separate Seyferts, LINERs and normal galaxies (see Table 3.2). In the bottom row, the Kewley et al. (2001) star formation line (black line) and the Kewley et al. (2006) Seyfert-LINER separation line (green line) are used to separate our sample galaxies into HII, Seyferts and LINERs/Transition objects types. 
The differences between Ho and Kewley nuclear classifications are mainly due to the criteria they used to distinguish the different objects. Ho et al. (1997) used simple criteria in term of emission line ratios to classify the galaxies as shown in table 3.2. Kewley et al. (2001) and Kewley et al. (2006) took into account stellar population synthesis and photoionization models to distinguish H II region-like galaxies to active galaxies. To separate Seyfert from LINER galaxies, they find the best-fit curve between the two AGN branches in the $[\mathrm{O} \mathrm{III}] / \mathrm{H} \beta$ versus $[\mathrm{S} \mathrm{II}] / \mathrm{H} \alpha$ and $[\mathrm{O} \mathrm{III}] / \mathrm{H} \beta$ versus $[\mathrm{O} \mathrm{I}] / \mathrm{H} \alpha$ diagnostic diagrams (Kewley et al., 2006). Therefore, the criteria used by Kewley et al. (2001) and Kewley et al. (2006) to determine the spectral classification of the galaxies are more physical and robust than the Ho et al. (1997) ones.

The second difference between the two classifications comes from the line ratios used to define them. Kewley et al. (2006) used galaxies of redshift $\gtrsim 0.04$ in order to have at least $20 \%$ of the total flux of the galaxy enclosed within the fiber, required to determine robust spectroscopic properties (Kewley et al., 2005). They extracted then spectral properties in apertures from $\sim 2.3 \mathrm{kpc}$ to $\sim 7 \mathrm{kpc}$ in diameter. Ho et al. (1997) used a $2^{\prime \prime} \times 4^{\prime \prime}$ aperture to extract the spectral properties of their sample of galaxies. This aperture size is similar to the $3^{\prime \prime}$ fiber aperture of the SDSS used by Kewley et al. (2006) but the galaxies studied by Ho et al. (1997) are closer to those in the Kewley et al. (2006) sample. Therefore, Ho et al. (1997) apertures range from $\sim 7 \mathrm{pc} \times 14 \mathrm{pc}$ to $\sim 1 \mathrm{kpc} \times 2 \mathrm{kpc}$. The maximum aperture covering fraction of Ho et al. (1997) is then of about $10 \%$.

Large apertures may include emission from extranuclear $\mathrm{H}$ II regions, therefore emission lines ratio used in diagnostic diagrams are smaller for larger aperture sizes (Storchi-Bergmann, 1991, as shown for the [N II]/ $\mathrm{H} \alpha$ ratio by). An active galaxy may then be misclassified to $\mathrm{H}$ II region-like object if its spectral properties are extracted from large aperture. Therefore, all the emission lines ratio used by Ho et al. (1997) and in this study should be smaller.

Fig. 3.3 is similar to fig. 3.2 and shows the nuclear classifications of the 16 ambiguous objects. There are three categories of misclassified galaxies:

- Galaxies that are classified as H II galaxies under one classification and as LINER or transition objects under the other. Four of the 16 ambiguous objects fall into this category. In this case, the differences between the Ho et al. (1997) and Kewley et al. (2001, 2006) classifications do not change our sample definition: these four galaxies still remain in our control sample.

- The second category includes galaxies that are classified as LINER galaxies or transition objects under one classification and as Seyferts under the other one. Half of the ambiguous objects (9/16) falls into this category.

- Finally three galaxies (NGC 3655, 4698 and 4826) are classified as H II galaxies under the Ho et al. (1997) criteria and as Seyferts under the Kewley et al. $(2001,2006)$ criteria. 
The 12 objects in the last two categories would be classified as active galaxies (Seyferts) or non-active (H II, LINER or transition objects) depending on the chosen criteria. In the following I will describe the cases of these objects and conclude on their chosen nuclear classification.

- Three of these objects (NGC 3627, 3489 and 3655) are misclassified because of the simple criteria of Ho et al. (1997). They are all Seyfert galaxies, lying very close to the Ho et al. (1997) LINER/Seyfert or H II / Seyfert limits, and indeed Ho et al. (1997) classified them between Seyfert and H II or transition objects. When using the Kewley et al. $(2001,2006)$ criteria (see bottom right panel of fig. 3.3), they are well located in the Seyfert regions. They can finally remain in our sample of active galaxies.

- Four galaxies (IC 356, NGC 2967, NGC 2859 and NGC 4826) seem to have very uncertain nuclear classification with both the Ho et al. (1997) and the Kewley et al. $(2001,2006)$ criteria. The three cases of IC 356, NGC 2967 and NGC 4826 are similar. These objects belong to our non-active galaxies sample. They are however classified as Seyferts in the [O III] / $\mathrm{H} \beta$ versus [O I] / H $\alpha$ diagram by Kewley et al. $(2001,2006)$ and as H II or LINER objects in the other diagram by Ho et al. (1997) and Kewley et al. (2001, 2006). These objects are located close to the Seyfert/LINER boundary of Kewley et al. (2006) or to the Kewley et al. (2001) H II / Seyfert boundary (fig.3.3 bottom right panel), therefore we consider them as transition or $\mathrm{H}$ II objects and they remain in the non-active galaxies sample. In the case of NGC 2859, it seems more ambiguous. This galaxy is classified as LINER by Ho et al. (1997) but lies not far from their LINER/Seyfert boundaries and while it is located in the LINER region defined by Kewley et al. (2006) in the [O III] / $\mathrm{H} \beta$ versus [S II] / $\mathrm{H} \alpha$ diagram, it is classified as Seyfert with no ambiguity in the $[\mathrm{O}$ III] / $\mathrm{H} \beta$ versus [O I] $/ \mathrm{H} \alpha$ diagram. If we take into account the fact that the line ratios are extracted inside a small aperture, this galaxy should lie in the bottom left side of its actual position in all the diagrams. However since we have no data to extract spectral information from larger aperture for this galaxy, we cannot conclude about its classification.

- The five remaining galaxies (NGC 2655, 3031, 4579, 4639 and 7331) seem to be misclassified by Ho et al. (1997). NGC 3031 and NGC 4639 are located in the LINER region of the diagnostic diagrams with the Kewley et al. $(2001,2006)$ criteria. They have been originally classified as LINER galaxies (Heckman, 1980) and Chiaberge et al. (2005) reported that NGC 3031 and NGC 4639 lie in the LINER region of their optical nuclear luminosity versus radio core luminosity diagram (see their Fig.7). NGC 4579 is classified as S1.9/L1.9 by Ho et al. (1997) and lies very close to the LINER/Seyfert boundaries. This galaxy is classified as LINER without ambiguity by Kewley et al. (2001, 2006). These three galaxies (NGC 3031, 4639 and 4579) seem then to be either low luminosity Seyfert galaxies, or either high energy LINER galaxies. In the two cases, we can consider them as active galaxies and place them in our active sample. 
NGC 2655 is a well known Seyfert 2 galaxy but is classified as LINER with the Kewley et al. $(2001,2006)$ criteria. Such classification may arise because of the lines ratio used to construct the diagrams are divided from small aperture size. Using a larger aperture size, covering $20 \%$ of the galaxy light as required by Kewley et al. (2005) would shift NGC 2655 in the bottom left side of the diagnostic diagrams, putting it in the Seyfert regions defined by Kewley et al. (2006). We then consider NGC 2655 as a Seyfert galaxy.

At last, NGC 7331 seem to be truly misclassified as a transition object by Ho et al. (1997). This galaxy lies very close to the Ho et al. (1997) limits between Seyferts and LINERs and it is located well within the Seyfert regions defined by the Kewley et al. (2001) and Kewley et al. (2006) boundaries. As for the case of NGC 2859, if we take into account small aperture used to extract the line rations, this galaxy may be a $\mathrm{H}$ II regionlike galaxy which has been misclassified as transition object by Ho et al. (1997) and Seyfert by Kewley et al. (2006). However as for NGC 2859, we cannot conclude about its classification.

Finally, among the 16 objects with ambiguous classifications, only two galaxies seem to have been truly misclassified in our study: NGC7331 and NGC 2859. This represents $2.5 \%$ of our total sample. We then conclude that using the Ho et al. (1997) nuclear classification, rather than the more recent Kewley et al. $(2001,2006)$ one, does not affect the definition of our active and non-active samples. We can then consider that any properties of the active sample that would be found in our study is representative of active galaxies and can be compared to those of our inactive sample.

\subsubsection{The VHIKINGS and SAURON/Seyfert samples}

Two sub-samples were then constructed from our master sample, corresponding to the VHIKINGS and the SAURON/Seyfert surveys:

\section{- The VHIKINGS sample.}

The VHIKINGS survey (VLA Hydrogen Imaging and Kinematics of INactive Galaxies and Seyferts) is a high angular resolution $\left(20^{\prime \prime}\right) \mathrm{HI}$ synthesis imaging spectroscopic survey of 56 Seyfert and inactive control galaxies, selected from our master sample. They correspond to the 26 brightest Seyferts (absolute nuclear V-band magnitudes $<-17.2$ ) and their associated inactive galaxies, plus two fainter Seyferts (NGC5194 and NGC 3031) and their control galaxies. The VHIKINGS sample is then composed of the 28 first pairs of Seyfert/non active galaxies listed in Table A.1. Figs. B.1 to B.4 (Appendix B.1) show the R-band Digital Sky Survey (DSS) images of the galaxies of the VHIKINGS sample.

\section{- The SAURON/Seyfert sample}



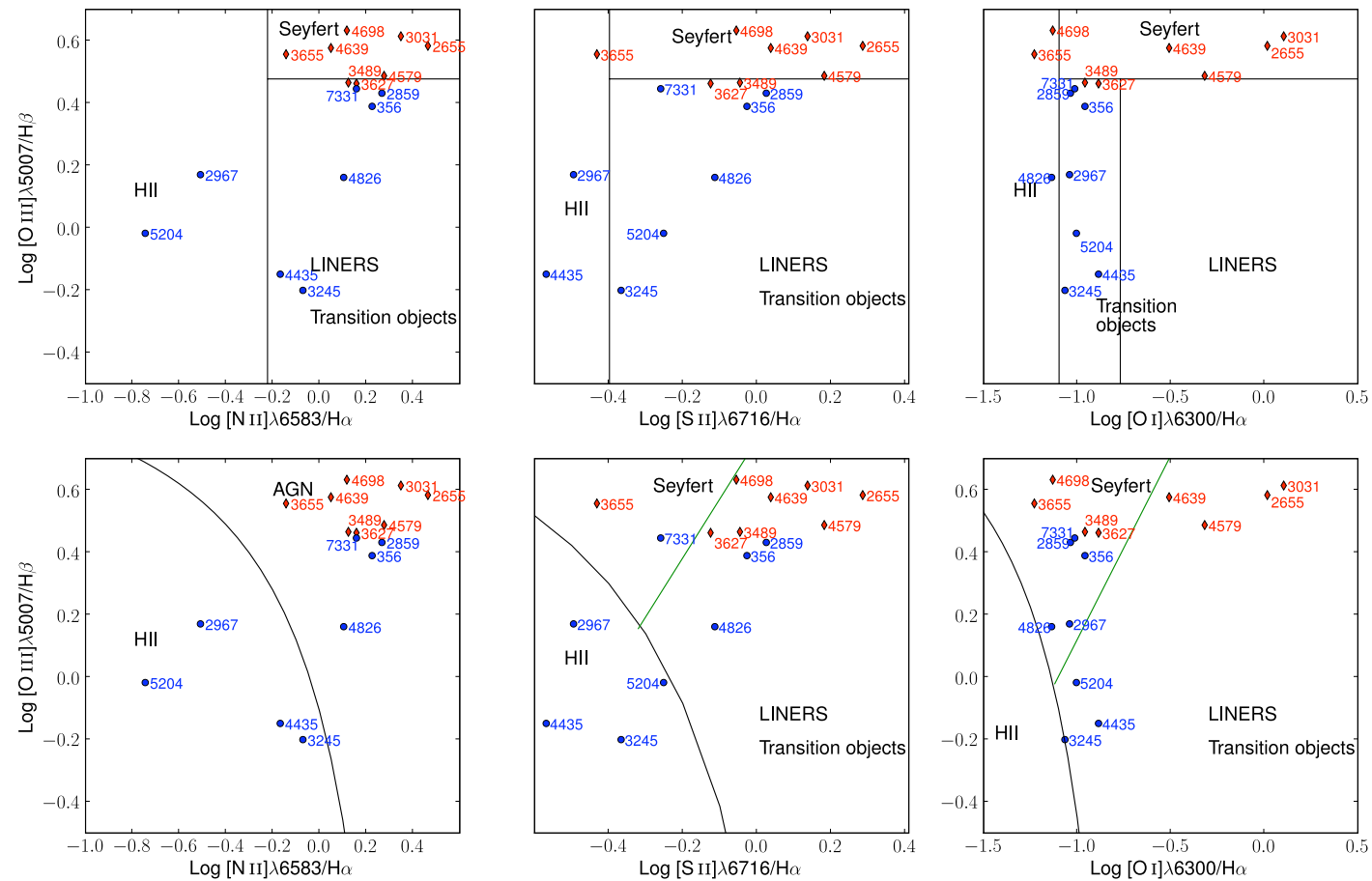

Figure 3.3: The $[\mathrm{O} \mathrm{III}] / \mathrm{H} \beta$ versus $[\mathrm{OI}] / \mathrm{H} \alpha$ (top panels) and [O III] $/ \mathrm{H} \beta$ versus [N II] / H $\alpha$ (bottom panels) diagnostic diagrams for the 16 ambiguous galaxies. The red diamonds represent the galaxies considered as Seyfert in our studies and the blue points correspond to their control non-active galaxies. Near each point, lies the NGC name of the corresponding galaxy. As for fig.3.2, the Kewley et al. (2001) star formation line (black line) and the Kewley et al. (2006) Seyfert-LINER separation line (green line) are used to separate our sample galaxies into HII, Seyferts and LINERs/Transition objects types. in the two left panels, while the boundaries from Ho et al. (1997) are used to separate Seyferts, LINERs and normal galaxies in the right panels. 
For our optical study via the SAURON IFU, we selected a distance-limited sub-sample of Seyfert/control galaxies from the master sample, which forms then the SAURON/Seyfert sample. The galaxies of this sample have $V_{\text {sys }}<1600 \mathrm{~km} \mathrm{~s}^{-1}$ to ensure that Fe stellar absorption lines lie well within the spectral band for the full SAURON field-of-view. Measurement of these Fe lines is critical in order to determine the stellar component morphology and kinematics in the presence of emission lines from ionised gas that contaminate $\mathrm{Mg}$ lines (see Sec. 3.2.2). In total, our subsample comprises 15 pairs of Seyferts+inactive galaxies. So far, we completed observations of 7 pairs and two well-known Seyferts (NGC 1068 and NGC 3227) with no control galaxy data. NGC 1068 has been studied in detail by Emsellem et al. (2006) and is included here for completeness, while weather constraints prevented observations of the control galaxy for NGC 3227. Table 3.3 lists the properties of the sample galaxies. The Vband absolute magnitudes of the Seyfert nuclei in this sub-sample span the full magnitude range of the 25 brightest Seyfert nuclei in the RSA catalogue $\left(-20.9<M_{V}<-16.0\right)$, thus offering a representative selection of Seyfert activity. Fig. 3.4 presents the SAURON field-of-view overlaid on R-band Digital Sky Survey (DSS) images of the 15 observed galaxies. 


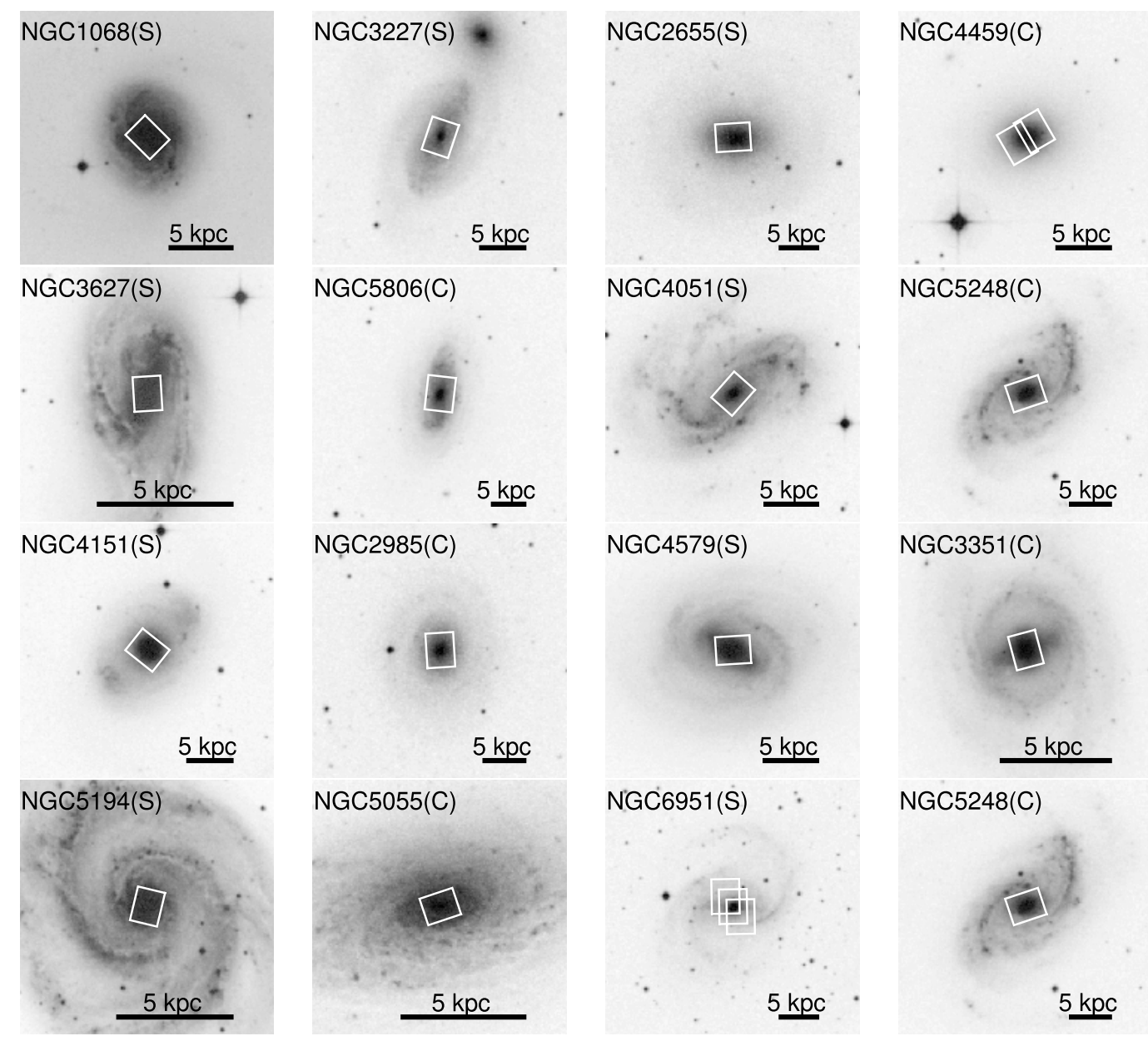

Figure 3.4: R-band Digital Sky Survey images of the SAURON sample galaxies. The '(S)' or ' $(C)$ ' on the right of the object names stand for Seyfert or Control galaxy, respectively. Each Seyfert galaxy is displayed on the left of its associated control galaxy, except for NGC 1068 and NGC 3227 (first two panels) for which no control has been observed. The orientation is such that north is up and east is left. The bar located at the bottom right corner of each panel corresponds to the spatial length of $5 \mathrm{kpc}$. Overplotted on each image is the position of the SAURON field of view for that galaxy. 


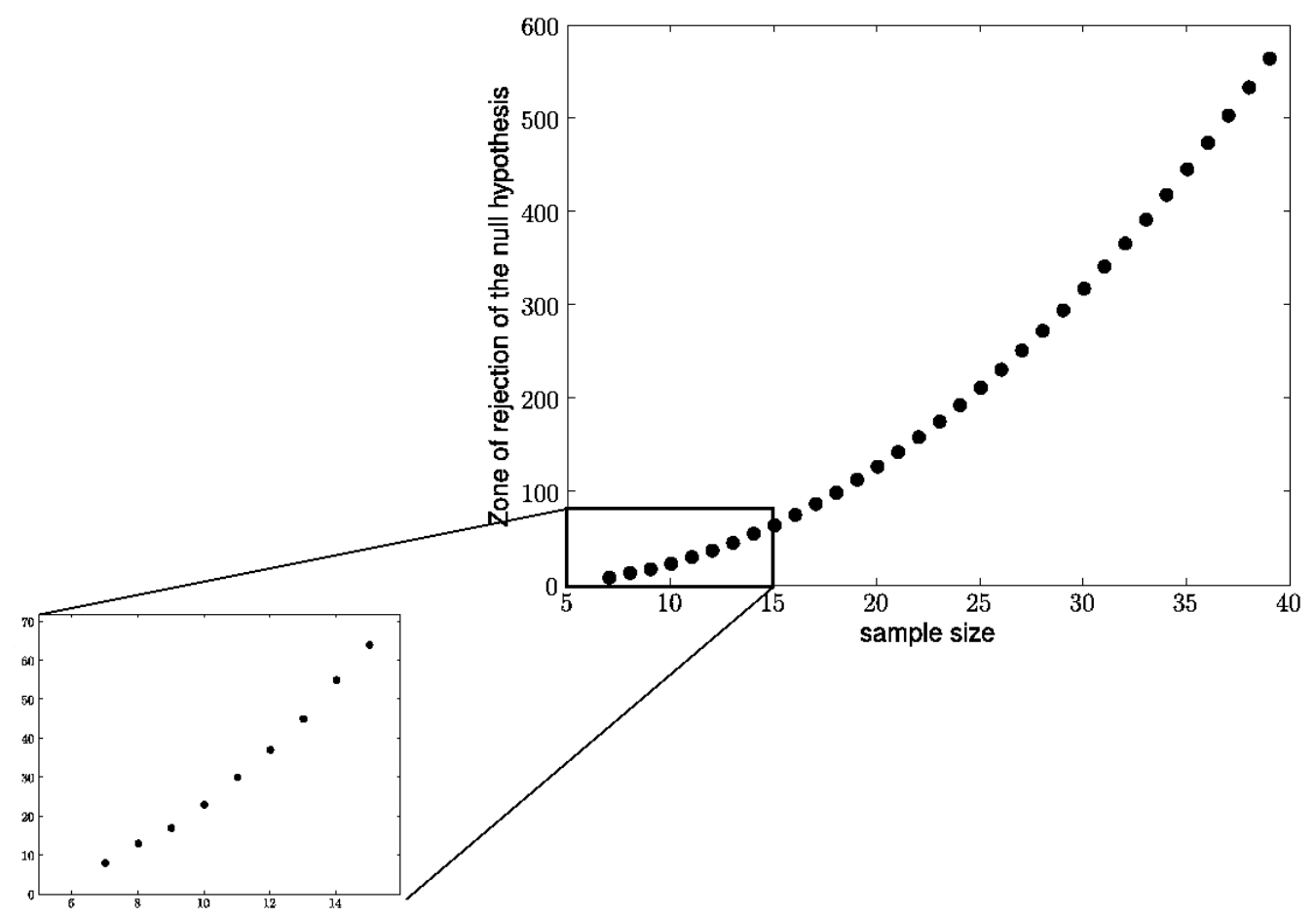

Figure 3.5: Zone of rejection of the null hypothesis as a function of the sample size. The black dots correspond to the maximum value of the statistic $U$ (see Sec. 5.1.3) for which the null hypothesis can be rejected. The zone of rejection of the null hypothesis corresponds to values of $U$ below this curve.

The downsizing of our sample from 39 pairs in the parent sample to 7 pairs in the SAURON sample implies certainly some consequences on the statistic of our results. We are investigating the kinematics of the circumnuclear regions of Seyfert and non-active galaxies, and in particular we are comparing a sample of Seyfert galaxies paired carefully with non-active galaxies in order to detect any kinematic difference between these two population, in the stellar and gaseous components. Considering the kinematic misalignment between the stars and ionised gas (see Sec. 5.1.2 for details), we can plot the zone of rejection of the null-hypothesis that these two samples are from the same distribution. The Mann Whitney $U$ test is based on the statistic variable $U$ (see Sec. 5.1.3). The zone of rejection of the null-hypothesis via the U test at a chosen level of significance depends on the sample size. The fig. 3.5 presents the maximum values of $U$ for which we can statistically distinguish the two samples at a level of significance of 5\%. At a given sample size, for all values of $\mathrm{U}$ below this maximum, we can conclude that the 2 samples are statistically different with a probability higher than $95 \%$. As we can see on this figure, this zone is much smaller for 7 pairs than 28 pairs. Therefore, the error to accept a wrong null-hypothesis is larger for small samples, as in our case. We will then ought to interpret the result of any statistical test on our data with great care. 


\begin{tabular}{|c|c|c|c|c|c|c|c|c|c|}
\hline$\stackrel{+}{\mathscr{D}} \quad \widehat{\Xi}$ & - & $N$ & $n \infty$ & $\infty+$ & $\infty$ & $10 \mathrm{~cm}$ & $\infty$ & $\infty \pi$ & a 10 \\
\hline 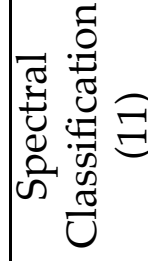 & $\mid \infty$ & مُ & कै & 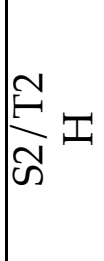 & $\frac{\sim}{\omega}$ I & एक & 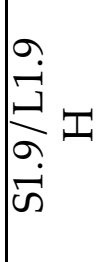 & W $N$ & ஸे I \\
\hline 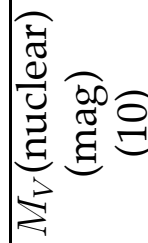 & 足 & \begin{tabular}{l}
$\infty$ \\
$\infty$ \\
\hdashline \\
\end{tabular} & 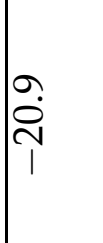 & $\frac{N}{1}$ & $\mid \begin{array}{l}2 \\
\infty \\
-1 \\
1\end{array}$ & $\frac{\hat{\sigma}}{2}$ & مั: & $\begin{array}{l}0 \\
0 \\
1\end{array}$ & م् \\
\hline 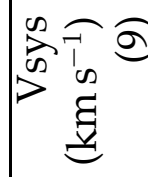 & 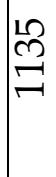 & I & 잉 & $\hat{N}$ 命 & 욧 & 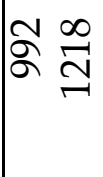 & 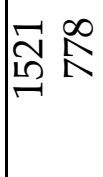 & \begin{tabular}{ll}
3 & 4 \\
0 & 5 \\
\hdashline & 10
\end{tabular} & 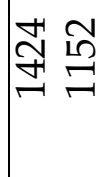 \\
\hline مी & a & 을 & ڤ̊ & $\mid \begin{array}{ll}10 & ? \\
0 & + \\
\sigma & \text { d }\end{array}$ & 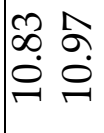 & 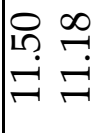 & 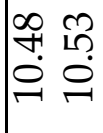 & $\mid \begin{array}{cc}0 & \bar{\sigma} \\
\infty & \sigma \\
\infty & \sigma\end{array}$ & 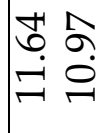 \\
\hline 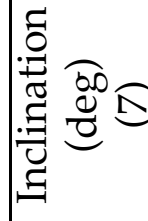 & શે & 10 & लि & గا & 앙 아 & $\vec{\sim} \stackrel{\infty}{\infty}$ & 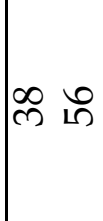 & ○ิ மீ) & m 아 \\
\hline 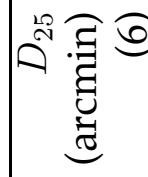 & ب. & مि & 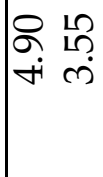 & 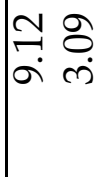 & 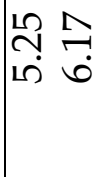 & 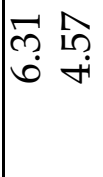 & 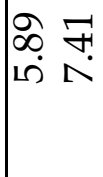 & 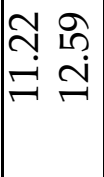 & 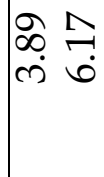 \\
\hline 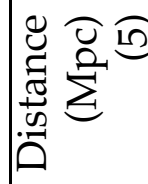 & $\stackrel{+}{+}$ & $\begin{array}{l}0 \\
\dot{\sim}\end{array}$ & $\begin{array}{ll}\forall & \infty \\
\stackrel{\sim}{+} & 0 \\
\end{array}$ & $\mid \begin{array}{ll}0 \\
0 \\
0\end{array}$ & 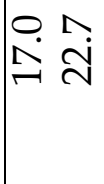 & 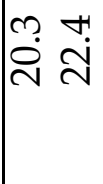 & $\mid$\begin{tabular}{ll}
$\infty$ & \multicolumn{1}{c}{} \\
\hdashline & $\infty$
\end{tabular} & 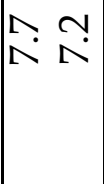 & 고 \\
\hline$H \quad \Im$ & $\infty$ & $\stackrel{r}{r}$ & 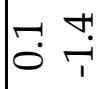 & $\begin{array}{cc}0 & m \\
\ddot{\theta} & \ddot{n}\end{array}$ & $\underset{+}{\sim} \underset{+}{\sim}$ & $N \sim$ & $\begin{array}{ll}\infty & \ddots \\
\sim & \hat{v}\end{array}$ & $\underset{+}{\mathrm{H}}+$ & ले \\
\hline م & 产 & 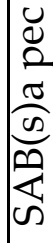 & 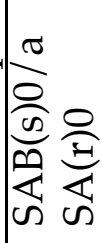 & 空兽 & 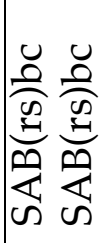 & 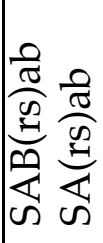 & 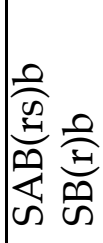 & 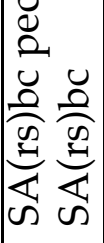 & 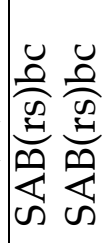 \\
\hline 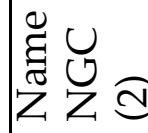 & & సิ & 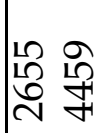 & 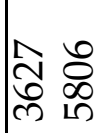 & 롱 & 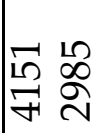 & 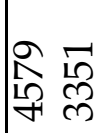 & 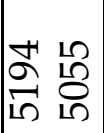 & 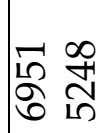 \\
\hline$\stackrel{\infty}{\sigma}$ & & & $r$ & $N$ & $m$ & 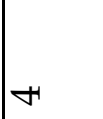 & 10 & 6 & \\
\hline
\end{tabular}

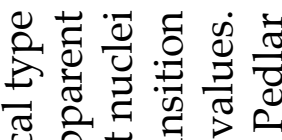

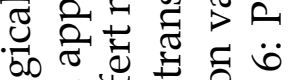
퓨유

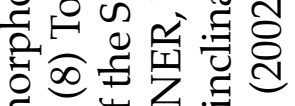
छे त्ष

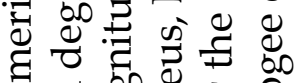

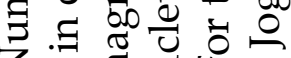
Z ⿷匚 己

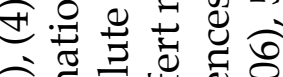

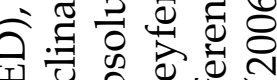

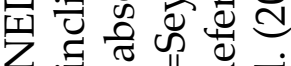

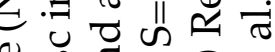
ڤ.

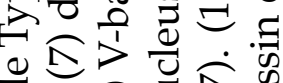
츠을

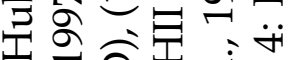

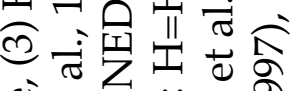

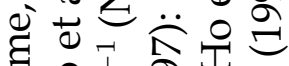
ฮำ

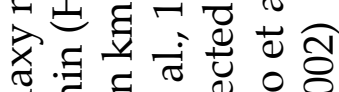
ส ป d สิ $\cong 0 ّ$ सं 氖 -

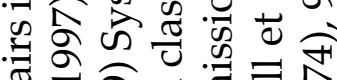

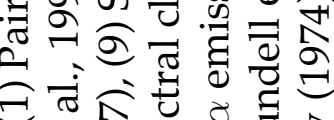
ن प बे की

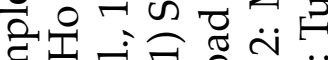

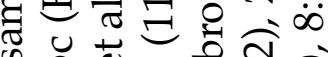
क ठี

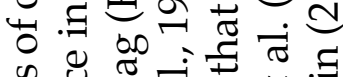

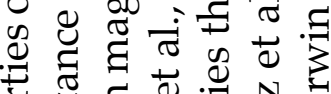

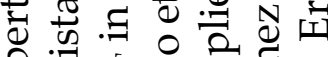

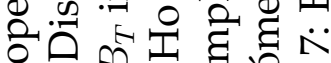

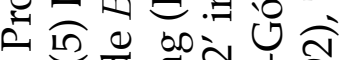
শั

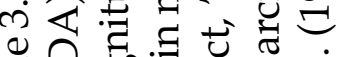
응

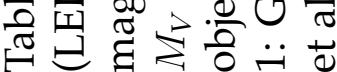




\subsection{Observation and data reduction}

In this Section, the observations parameters of the VHIKINGS and SAURON/Seyfert sample are presented. I develop then the reduction methods specific to the VLA and SAURON data.

\subsubsection{VHIKINGS survey}

\section{- Observations and archival data}

The VHIKINGS survey aims at investigating the large-scale disk H I dynamics and detecting any gas-rich satellites with low surface brightness. The $\mathrm{C}$-array configuration was chosen as the optimal compromise between spatial resolution (size of the synthesised beam, $\sim 20^{\prime \prime}$ in the case of the C-array configuration) and the largest angular scale (LAS) which is the size of the largest structure seen. Table 3.4 lists the different synthesised beam size and LAS for the different array configurations in the L-Band (corresponding to wavelength of $20 \mathrm{~cm}$ ).

First of all, we checked whether suitable data were available within the VLA on-line archives for our galaxies. We choose archival data in the L band and with array configuration as close as possible to the $C$ configuration. We used archival data for 28 galaxies of the VHIKINGS sample and set up observations for the remaining 28 galaxies with no suitable or compromised archival $\mathrm{HI}$ data.

Between October 2002 and January 2007, 22 of these 28 galaxies have been observed, using the VLA in C array configuration. Spectral line mode 4 has been used with intermediate frequency (IF) bandwidth of $3.125 \mathrm{MHz}$ and 32 channels per IF. On-line Hanning smoothing gave then an effective velocity resolution of $25 \mathrm{~km} \mathrm{~s}^{-1}$. For maximum sensitivity and large spectral coverage, IFs have been combined in 2 pairs, giving effective bandwidth per pair of $\sim$ $1000 \mathrm{~km} \mathrm{~s}^{-1}$. This instrumental set-up ensures a sensitivity to column densities $N_{H}>10^{20} \mathrm{~cm}^{-2}$ and a bandwidth corresponding to $\Delta V=1100 \mathrm{~km} \mathrm{~s}^{-1}$. Such broad bandwidth and high sensitivity allows to detect gas-rich dwarf galaxies with low optical surface brightness and associated with the targeted galaxies.

Finally we have data (from the archives or our specific observations) for all but six galaxies of the VHIKINGS sample (NGC 2950, 3245, 3504, 3516, 5204 and 5273), for which no suitable archival data are available and which could not be observed as part of the VHIKINGS survey. Table B.1 lists the observation parameters for the available data. 


\begin{tabular}{|c|c|c|c|}
\hline \multicolumn{4}{|c|}{ configuration } \\
\hline A & B & C & D \\
\hline \multicolumn{4}{|c|}{ synthesised beam } \\
\hline $1.4^{\prime \prime}$ & $3.9^{\prime \prime}$ & $12.5^{\prime \prime}$ & $44^{\prime \prime}$ \\
\hline \multicolumn{4}{|c|}{ LAS } \\
\hline $38^{\prime \prime}$ & $2^{\prime}$ & $15^{\prime}$ & $15^{\prime}$ \\
\hline
\end{tabular}

Table 3.4: Configuration synthesised beam sizes and largest angular scales (LAS) for the L band.

\section{- Data Reduction}

All data processing was performed using the NRAO Astronomical Image Processing System (AIPS). Calibration and initial data editing were carried out using the pseudo-continuum channel (channel 0) which is formed on-line by averaging the central $75 \%$ of the bandwidth. The resulting phase and gain calibration solutions were applied to the spectral line data and these data were then Fourier transformed with natural weighting to optimise the surface brightness sensitivity. A continuum image was formed by averaging several channels free of line emission, then this continuum image was subtracted from the corresponding spectral-line cube to form a continuum-free cube. Finally, the continuum image and continuum free cube were deconvolved with the AIPS task IMAGR.

The first three moments are then calculated from the continuum-free cube. The zeroth moment corresponds to the integrated intensity over the channels, the first moment to the intensity-weighted velocity and the second moment to the intensity-weighted velocity dispersion. Several methods exist to derive the moments map from spectral line cubes. In this work, the moment maps were derived using automatic routine in AIPS such as XMOM or MOMNT.

\subsubsection{SAURON/Seyfert survey}

\section{- Observations}

Observations of the SAURON/Seyfert sub-sample were carried out between 2001 and 2004 using the integral-field spectrograph SAURON at the $4.2 \mathrm{~m}$ William Herschel Telescope (WHT) at La Palma, Spain. Table 3.5 summarises the exposure time for each pointing.

The low spatial resolution mode of SAURON was used, providing a FOV of $33^{\prime \prime} \times 41^{\prime \prime}$ with a square sampling of 0.94 arcsec per spatial element (lens). This delivers 1431 spectra simultaneously per object and 146 spectra of a region about 2 arcmin away from the pointing, allowing to measure the sky background. Each spectrum covers the spectral range $4825-5380 \AA$, with a resolution of $4.2 \AA$ (FWHM). This wavelength range includes a number of important 


\begin{tabular}{|c|c|c|c|}
\hline $\begin{array}{l}\text { Pairs } \\
\text { (1) }\end{array}$ & $\begin{array}{l}\text { Name } \\
\text { NGC } \\
\text { (2) }\end{array}$ & $\begin{array}{l}\text { dates } \\
\text { (3) }\end{array}$ & $\begin{array}{l}T_{\exp } \\
\text { (s) } \\
\text { (4) }\end{array}$ \\
\hline & 1068 & 2002 January & $300+3 \times 1800$ \\
\hline & 3227 & 2004 March & $3 \times 1800$ \\
\hline 1 & $\begin{array}{l}2655 \\
4459\end{array}$ & $\begin{array}{l}2004 \text { March } \\
2001 \text { March }\end{array}$ & $\begin{array}{l}6 \times 1800 \\
4 \times 1800\end{array}$ \\
\hline 2 & $\begin{array}{l}3627 \\
5806\end{array}$ & $\begin{array}{l}2004 \text { March } \\
2004 \text { March }\end{array}$ & $\begin{array}{l}4 \times 1800 \\
2 \times 1800\end{array}$ \\
\hline 3 & $\begin{array}{l}4051 \\
5248\end{array}$ & $\begin{array}{l}2004 \text { March } \\
2004 \text { March }\end{array}$ & $\begin{array}{l}6 \times 1800 \\
5 \times 1800\end{array}$ \\
\hline 4 & $\begin{array}{l}4151 \\
2985\end{array}$ & $\begin{array}{l}2004 \text { March } \\
2004 \text { March }\end{array}$ & $\begin{array}{l}3 \times 1800 \\
3 \times 1800\end{array}$ \\
\hline 5 & $\begin{array}{l}4579 \\
3351\end{array}$ & $\begin{array}{l}2004 \text { March } \\
2004 \text { March }\end{array}$ & $\begin{array}{l}4 \times 1800 \\
4 \times 1800\end{array}$ \\
\hline 6 & $\begin{array}{l}5194 \\
5055\end{array}$ & $\begin{array}{l}2004 \text { March } \\
2004 \text { March }\end{array}$ & $\begin{array}{l}4 \times 1800 \\
6 \times 1800\end{array}$ \\
\hline 7 & $\begin{array}{l}6951 \\
5248\end{array}$ & $\begin{array}{l}2003 \text { August } \\
2004 \text { March }\end{array}$ & $\begin{array}{l}3 \times 1800 \\
5 \times 1800\end{array}$ \\
\hline
\end{tabular}

Table 3.5: Details of the exposures of our sample. (1) Pairs identifier, (2) NGC number, (3) date of observation (4) Exposure time in sec.

stellar absorption lines $(\mathrm{H} \beta, \mathrm{Fe} 5015, \mathrm{Mg} b, \mathrm{Fe} 5270)$ and ionised gas emission lines $(\mathrm{H} \beta,[\mathrm{O} \mathrm{III}]$ and $[\mathrm{NI}])$. Table 3.6 lists the different characteristics of the SAURON spectrograph. More details on SAURON can be found in Bacon et al. (2001).

\section{- Data reduction}

This section focuses on the reduction of data obtained with the integralfield spectrograph SAURON. The data of the 15 galaxies observed with SAURON were reduced using the XSAURON software and an automatic pipeline available within the SAURON consortium (Bacon et al., 2001; de Zeeuw et al., 2002). The main steps include: bias and dark subtraction, extraction of the spectra using a mask, wavelength calibration, low-frequency flatfielding, cosmic rays removal, homogenisation of the spectral resolution in the field, sky subtraction (using 146 sky spectra 1.9 arcmin away from the main field). Then the flux calibration was applied as explained in detail by Kuntschner et al. (2006), and the flux calibrated individual exposures are accurately centred with respect to each others and merged. The final merged datacube is sampled onto a square grid with 0 ! $8 \times 0.0^{\prime \prime} 8$ pixels.

Finally, the point spread function (PSF) of each merged exposure was determined by comparing the SAURON intensity distribution, reconstructed by integrating all wavelengths, with HST/WFPC2 images. The SAURON PSF is 


\begin{tabular}{|c|c|c|}
\hline \multirow[t]{2}{*}{ Characteristic } & \multicolumn{2}{|c|}{ Mode } \\
\hline & LR & HR \\
\hline \multicolumn{3}{|c|}{ spatial characteristics } \\
\hline Spatial sampling & $0.94^{\prime \prime}$ & $0.27^{\prime \prime}$ \\
\hline Field-of-view & $33^{\prime \prime} \times 41^{\prime \prime}$ & $11^{\prime \prime} \times 9^{\prime \prime}$ \\
\hline spectral resolution (FWHM) & $4.2 \AA$ & $3.6 \AA$ \\
\hline Spectral window & 4810 & $350 \AA$ \\
\hline \multicolumn{3}{|c|}{ spectral characteristics } \\
\hline Wavelength coverage & \multicolumn{2}{|c|}{$4500-7000 \AA$} \\
\hline Number of object lenslets & \multicolumn{2}{|c|}{1431} \\
\hline Number of sky lenslets & \multicolumn{2}{|c|}{146} \\
\hline Grism & \multicolumn{2}{|c|}{514 lines $\mathrm{mm}^{-1}$} \\
\hline Spectral sampling & $1.1 \AA \mathrm{pix}^{-1}$ & $0.9 \AA$ pix $^{-1}$ \\
\hline Instrumental dispersion $(\sigma)$ & $105 \mathrm{~km} \mathrm{~s}^{-1}$ & $90 \mathrm{~km} \mathrm{~s}^{-1}$ \\
\hline Spectra separation/PSF ratio & 1.4 & 2.3 \\
\hline Important spectral features & \multicolumn{2}{|c|}{$\mathrm{H} \beta,[\mathrm{O} \mathrm{III}], \mathrm{Mg} b, \mathrm{Fe} \mathrm{I},[\mathrm{N} \mathrm{I}]$} \\
\hline \multicolumn{3}{|c|}{ technical characteristics } \\
\hline Calibration lamps & \multirow{5}{*}{\multicolumn{2}{|c|}{$\begin{array}{c}\mathrm{Ne}, \mathrm{Ar}, \mathrm{W} \\
\text { William Herschel } 4.2 \mathrm{~m} \\
\text { EEV } 122148 \times 4200 \\
13.5 \mu \mathrm{m} \\
\approx 35 / 15 \%\end{array}$}} \\
\hline Telescope & & \\
\hline Detector & & \\
\hline Pixel size & & \\
\hline Efficiency (instrument/total) & & \\
\hline
\end{tabular}

Table 3.6: SAURON instrumental characteristic. The LR and HR columns correspond to the low resolution and high resolution modes, respectively.

modeled with a single two-dimensional Gaussian, whose parameters are determined by minimising the differences between the HST/WFPC2 images, convolved by this Gaussian, and the SAURON reconstructed images. The values of the FWHM of the PSF derived by this method seem to be dominated by instrumental systematics 


\section{Derivation of the stellar kinematics}

In order to ensure the reliability of the stellar kinematics measurements, the merged datacubes were spatially binned using the Voronoi 2D binning algorithm of Cappellari \& Copin (2003), creating bins with a minimum signal-to-noise ratio $(\mathrm{S} / \mathrm{N})=60$ per bin.

Observed spectra of galaxies $G_{*}(\lambda)$ are usually assumed to be the convolution of the spectrum resulting from the combination of stellar spectra $S(\lambda)$, and a broadening function, the line-of-sight velocity distribution $(L O S V D(v))$ :

$$
G_{*}(\lambda)=S(\lambda) \otimes L O S V D(v) .
$$

The $L O S V D(v)$ corresponds to the distribution of the stars over line-ofsight velocities and $S(\lambda)$ is adjusted by a stellar template spectrum taken from libraries of natural or artificial stellar spectra, using characteristic absorptions features such as the Mgb blend. The LOSVD contains the stellar kinematic parameters and is often considered to be Gaussian, parameterised by the stellar mean velocity $V_{*}$ and velocity dispersion $\sigma_{*}$. In some cases, deviations from a pure Gaussian profile have to be taken into account. Then another Gaussian is added or non-Gaussian components are included in the fit.

Many different methods exist to determine the best-fitting LOSVD model. Some of these methods use Fourier-based techniques to recover the LOSVD from a deconvolution such as the Fourier correlation quotient (Bender, 1990) or the cross correlation fitting methods (Statler, 1995). Direct fitting techniques have also been used (Rix \& White, 1992). The latter involves a larger computational time but allows to easily exclude emission lines or bad pixels. Since the observed SAURON spectra are contaminated by ionised gas emission lines ( $\mathrm{H} \beta \lambda 4861$, [O III] $\lambda \lambda 4959,5007$ and $[\mathrm{N}$ I $] \lambda \lambda 5198,5200)$, I used a direct pixel fitting (pPXF) method developed by Cappellari \& Emsellem (2004) to measure the stellar kinematics for each spectrum. The implementation of this method for the SAURON data is detailed by Emsellem et al. (2004). The spectral regions contaminated by emission are first identified and correctly masked out as shown in Fig. 3.6. The spectra are then rebinned in $\ln \lambda$ and the algorithm finds the best fit to each galaxy spectrum by convolving a stellar template spectrum with the corresponding LOSVD, which is parameterised by GaussHermite series (Gerhard, 1993; van der Marel \& Franx, 1993). In this context, $\operatorname{LOSVD}(v)=\mathcal{G}_{V_{*}, \sigma_{*}}(v)\left[1+h_{3} H_{3}+h_{4} H_{4}\right]$ where the Hermite polynomials $H_{3}$ and $H_{4}$ estimate the differences between the observed LOSVD and a pure Gaussian profile $\mathcal{G}_{V_{*}, \sigma_{*}}(v)$. Fig. 3.7 shows the effect of the addition of these Hermite components to the LOSVD profile.

This fit is performed via the three steps of an iterative process:

- A first estimate of the LOSVD is done using a single star spectrum as template. Values of $V_{*}, \sigma_{*} h_{3}$ and $h_{4}$ are computed from this first iteration 


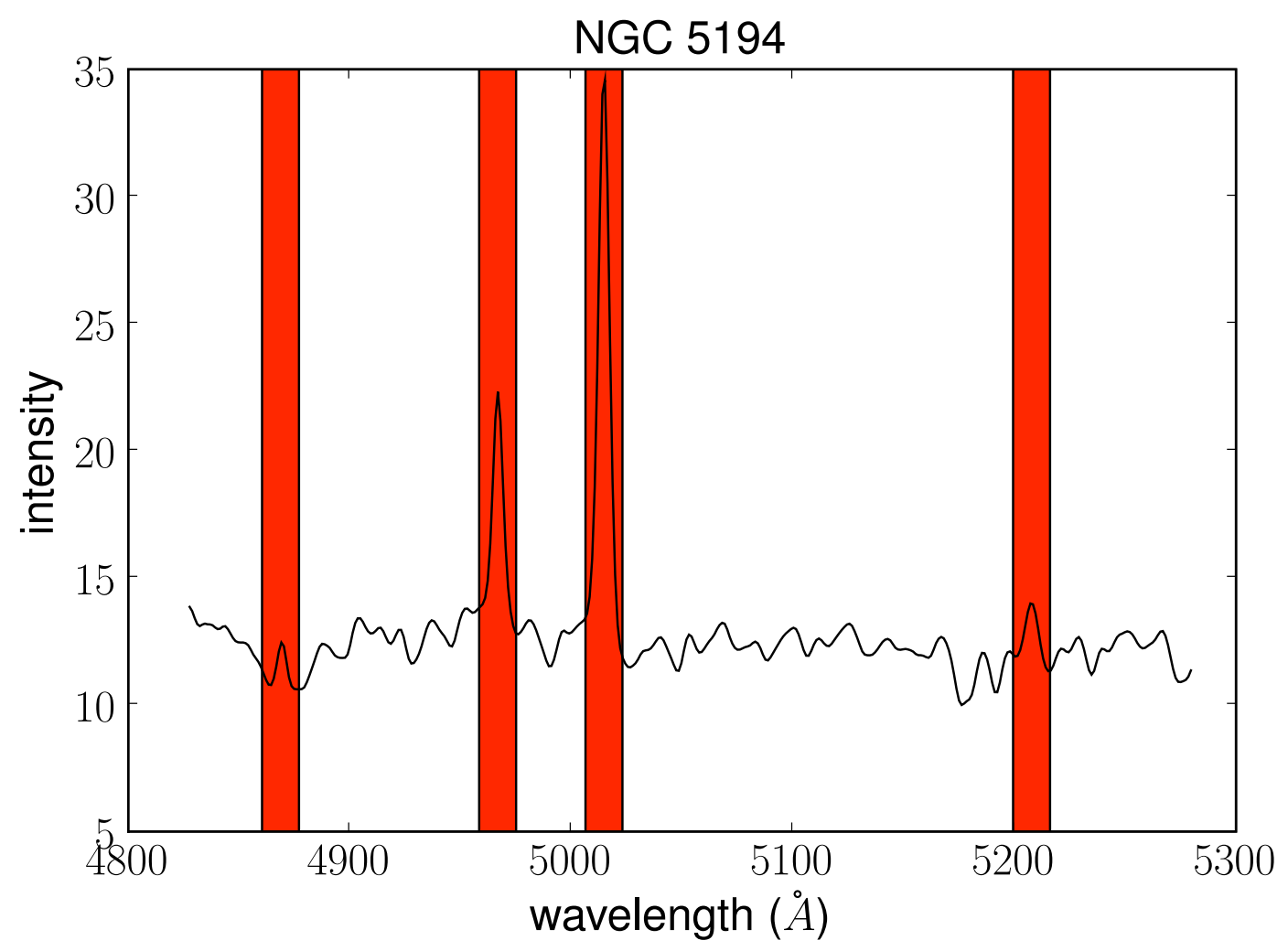

Figure 3.6: Central spectrum of NGC 5194. The spectral windows in red correspond to regions highly contaminated by ionised gas emission lines and they have been masked during the recovery of the LOSVD.

- Fitting the stellar kinematic parameters in the pixels space is very sensitive to mismatch between the template and the galaxy observed spectrum. Therefore, a stellar template closely matched to the galaxy spectrum is required. The second iteration of this process consists then of computing the optimal stellar templates, as linear combination of spectra from a stellar library, using the kinematic parameter estimated in the first step.

We used then a large stellar library spanning a wide range of ages and metallicities in order to derive optimal stellar templates for each individual spectrum. In this work, the library of single stellar population models of Vazdekis (1999) was chosen. A low-order polynomial is also included to account for small differences between the galaxy and the template spectra.

- Finally, the optimal template derived for each spectrum is used to measure best-fitting values of the kinematic parameters $V_{*}, \sigma_{*}, h_{3}$ and $h_{4}$. The errors are estimated by a Monte-Carlo method, in which the kinematic parameters are derived from several realisations of the input spectrum obtained by adding Poissonian noise to a model galactic spectrum. 

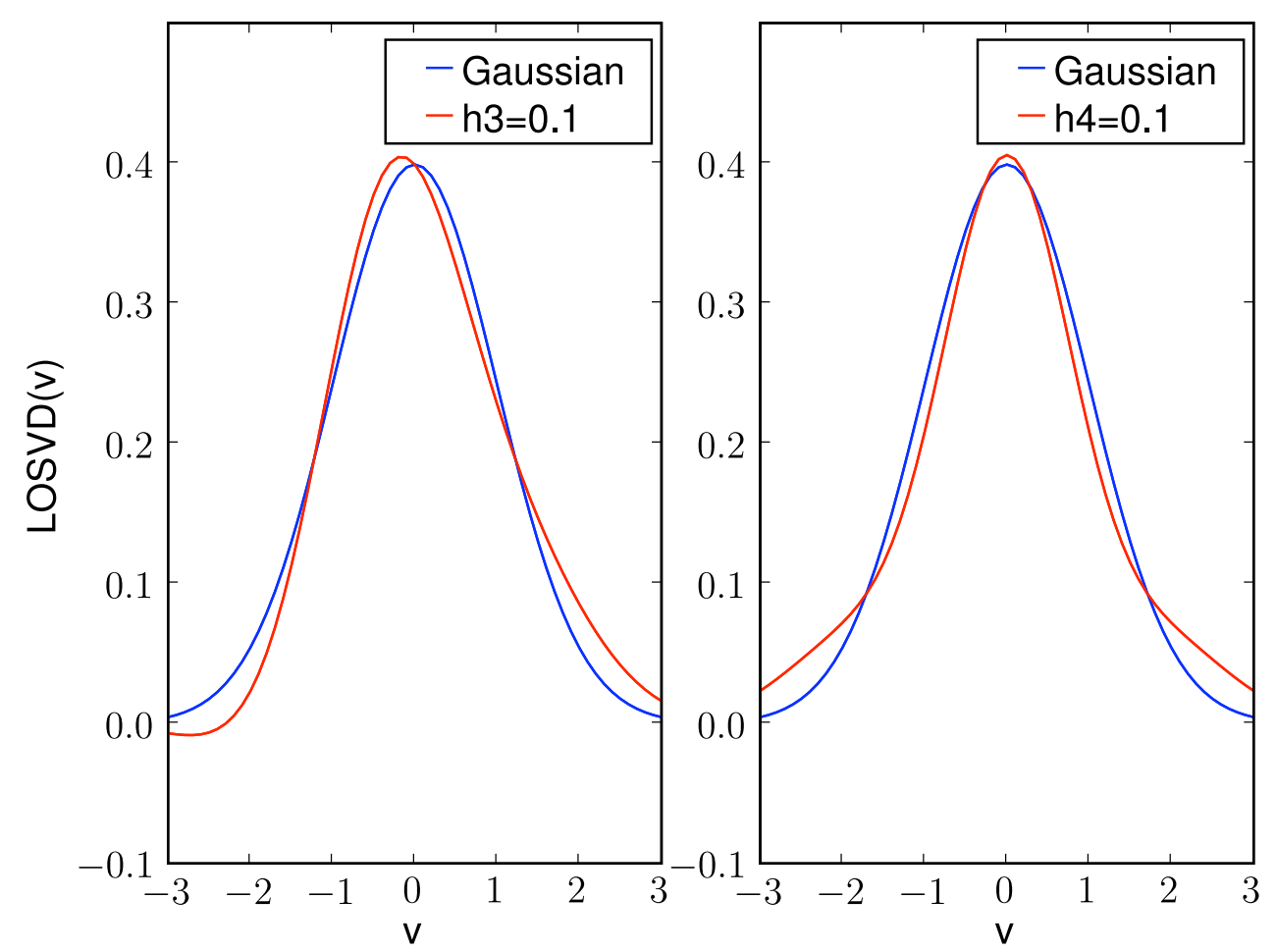

Figure 3.7: Contribution of the Hermite polynomials in the LOSVD profile. In the left plot, $h_{3} \neq 0$ and $h_{4}=0$. The peak of the LOSVD occurs at negative velocity for $h_{3}>0$ and positive velocity for $h_{3}<0$. This effect corresponds to the skewness of the profile. In the right plot, $h_{4} \neq 0$ and $h_{3}=0$. The profile of the LOSVD is more centrally peaked than a pure Gaussian profile for $h_{4}>0$ and is broader for $h_{4}<0$. This effect corresponds to the kurtosis of the profile.

The stellar kinematic parameters were determined for each individual spectrum using this method automatically implemented in the SAURON data reduction pipeline, for our whole sample except for the Seyfert 1 galaxies: NGC 3227, NGC 4051 and NGC 4151. For these galaxies, the automatic pipeline alone is inadequate due to the presence of a broad $\mathrm{H} \beta$ emission line in the inner few arcseconds (see Fig. 3.8.c). The spectral regions that are masked have to be carefully defined in order to exclude this broad component, and then we fit the stellar kinematics of these 3 galaxies interactively, using the same pixel fitting method.

The AGN continuum is not separated from the stellar component using this technique. The derivation of the stellar kinematics of the Seyfert $2 \mathrm{~s}$ is a priori not affected by the AGN continuum since the central engine is hidden from direct view. This continuum would however affect the derivation of the stellar velocity dispersion in the central regions of the Seyfert 1s, where it is dominant. These regions have been excluded from the following stellar kinematics analysis and subsequent discussions. 

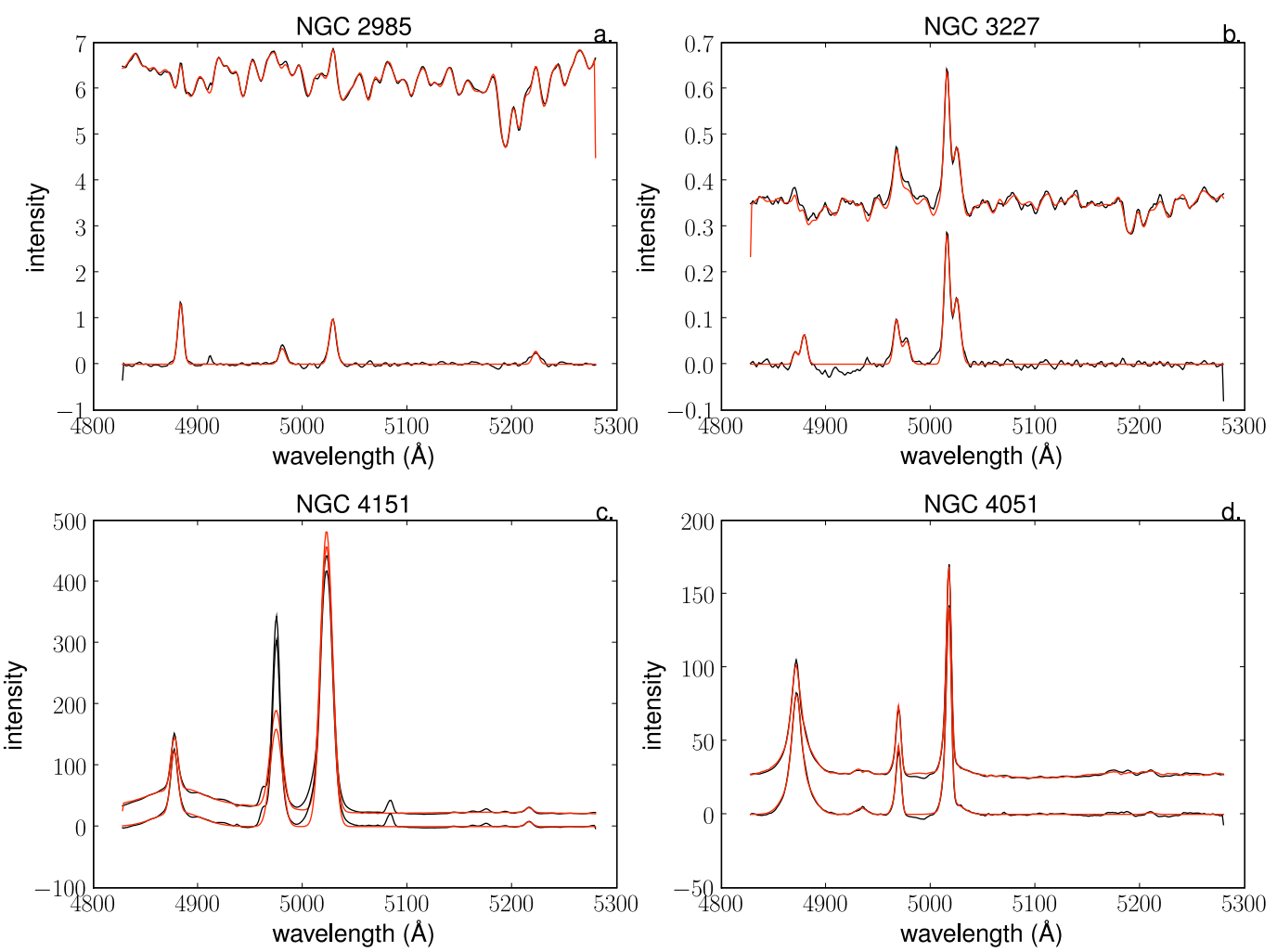

Figure 3.8: Examples of spectra and their corresponding fits for 4 galaxies. (a): NGC 2985 (inactive), (b): NGC 3227 (Seyfert 1.5), (c): NGC 4151 (Seyfert 1.5), (d): NGC 4051 (Seyfert 1.2). In each panel, the black line on the top shows the galaxy spectrum and the red line, its corresponding fit which is composed of the best-fitting stellar template added to the emission line fit. Below this spectrum, the pure emission line spectrum is shown in black. The corresponding emission lines fit is overplotted in red. The spectra were taken in the central pixel of the FOV, except for NGC 3227, for which the spectrum was taken at $4^{\prime \prime}$ North-East from the centre.

\section{Derivation of the gaseous distribution and kinematics}

The stellar continuum resulting from the stellar kinematic fit was subtracted from the original data, providing pure emission line datacubes. The wavelength range of our observations includes the $\mathrm{H} \beta \lambda 4861$, [O III] $\lambda \lambda 4959,5007$ and [N I] $\lambda \lambda 5198,5200$ emission lines. The parameters of these emission lines (intensity, mean velocity and FWHM) were derived from Gaussian fitting using the fit/spec software developed by Rousset (1992).

The fit was performed with two systems of emission lines: the first consisting of the $\mathrm{H} \beta$ and [N I] lines, and the second, of the [O III] lines. The $\mathrm{H} \beta$ and [O III] lines were fitted independently in order to detect differences in the kinematics of the two lines. Within each system, the lines were assumed to share the same velocity and FWHM. Constraints were applied on the parameters: the line ratio [O III] $\lambda 4959 /[\mathrm{O}$ III] $\lambda 5007$ was 

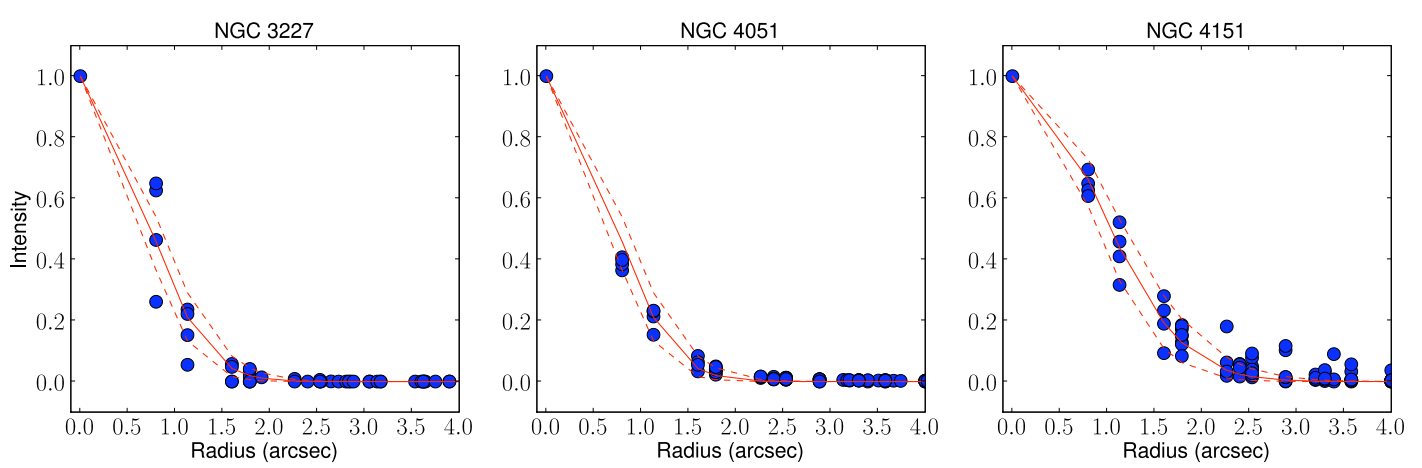

Figure 3.9: Radial normalised profiles of the BLR for NGC 3227, NGC 4051 and NGC 4151 (filled blue circles). The red solid line corresponds to the bestfit seeing value for each of the three galaxies, derived independently with HST images (see Table 3.5). The two red dashed curves correspond to the associated upper and lower limits at $2 \sigma$.

assumed throughout to be equal to 2.88. Moreover the velocity $\mathrm{V}$ and the velocity dispersion FWHM of the lines were bounded: V, around the systemic velocity of the galaxy, and the FWHM by the spectral resolution of SAURON FWHM SAURON $_{\text {SA }}=4.2 \AA$. Usually the line profiles were simple enough to be fitted automatically. A first fit was done using one single Gaussian profile and this automated fit has been visually controlled for each galaxy. An example of the emission lines fitting results is given in Fig. 3.8.a. A small number of galaxies show complex emission lines profiles and required a manual fit with additional emission line components. Additional components for [O III] lines, $\mathrm{H} \beta$ line or both were required in the case of five active galaxies: NGC 3227 (Fig. 3.8.b), 4051, 4151 and 5194. These additional components are certainly associated with the NLR of these galaxies. An unresolved central $\mathrm{H} \beta$ broad component has also been added in the case of the Seyfert 1 galaxies (NGC 3227, 4051 and 4151) which corresponds to the BLR. The FWHM of this component is $2600 \mathrm{~km} \mathrm{~s}^{-1}, 1500 \mathrm{~km} \mathrm{~s}^{-1}$ and $3100 \mathrm{~km} \mathrm{~s}^{-1}$ for NGC 3227, NGC 4051 and NGC 4151, respectively, and the spatial extent of the radial profile of the BLRs (Fig. 3.9) is consistent with the PSF derived above, within the derived uncertainties (Table 3.5). Finally, in the case of strong emission lines, a spectrum can be 'contaminated' by the closest neighbour spectrum. Therefore, extra emission lines are observed, at a wavelength shifted of $62.4 \AA$ which corresponds to the distance between two lenses in the detector plan. Fig. 3.10 shows a spectrum $2^{\prime \prime}$ west of the nucleus of NGC 4051. This spectrum presents $\mathrm{H} \beta$ and [O III] contamination lines (corresponding to the green labels in the figure.)

In addition to $\mathrm{H} \beta$ and [O III] lines, the [N I] $\lambda \lambda 5198,5200$ doublet is present in our SAURON spectra. Since the [N I] lines are significantly weaker than the $\mathrm{H} \beta$ and [O III] lines, their kinematics can not be constrained independently. Therefore this doublet is fitted together with the $\mathrm{H} \beta$ line system. $[\mathrm{N}$ I] lines share then the same kinematics as $\mathrm{H} \beta$ and we constrained the 


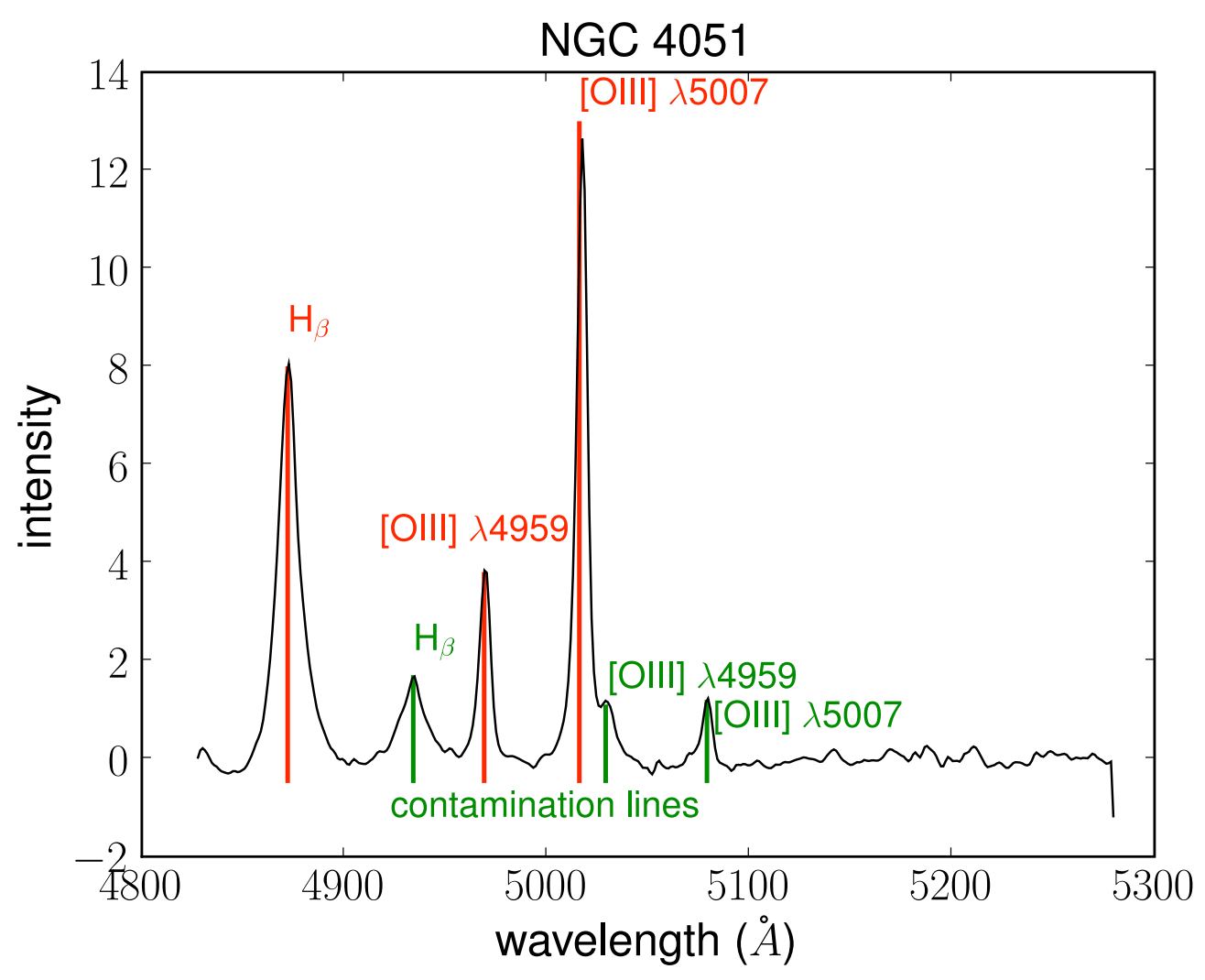

Figure 3.10: Example of spectrum with contaminated emission lines from the closest spectrum. The contaminated lines are shown with green labels, while the 'true' emission lines of this spectrum are labelled in red.

line ratio: $0.5<[\mathrm{N} \mathrm{I}] \lambda 5198 /[\mathrm{N}$ I $] \lambda 5200<1.5$. However, it appears that although the [N I] doublet is detected in most of our galaxies, it has a very low signal-to-noise. Therefore it is not discussed further. 


\section{Chapter 4}

\section{Results: the moment maps}

This chapter is dedicated to the presentation of the moment maps of the neutral, ionised gas and stars, for our sample of galaxies. The SAURON maps consist of the ionised gas and stellar distribution and kinematics within the central kpc regions of the 15 galaxies of the SAURON/Seyfert sample (Sec. 4.1). I present in Sec. 4.2 the H I moment maps corresponding to the 21 galaxies of the VHIKINGS sample for which data reduction has already been completed. Nine of these objects are also part of the SAURON/Seyfert sub-sample.

In this chapter, we discuss the general properties of the optical and radio maps. Detailed description of the SAURON and H I moments maps, and a comparison with previous published data for each galaxy can be found in Appendix E.

\subsection{The central regions of active and inactive galax- ies}

In this Section, we present the distribution and kinematic maps of the stellar and ionised gas components for our sample of galaxies. Figures C.1a to C.1h (Appendix C) present our SAURON maps: stellar continuum, [O III] and $\mathrm{H} \beta$ intensity distributions and emission line ratio $[\mathrm{O} \mathrm{III}] / \mathrm{H} \beta$, as well as stellar and gaseous kinematics (velocity and velocity dispersion). In the case of the Seyfert 1 galaxies, the line ratio $[\mathrm{O} I I] / \mathrm{H} \beta$ is computed using the narrow $\mathrm{H} \beta$ component. The galaxies are displayed by pair: the Seyfert on the top, its control galaxy below, except for NGC 1068 and NGC 3227 which are shown together (Fig. C.1a). All the maps are oriented so that the outer photometric major-axis of the galaxy is on the horizontal axis. To display the gas maps, we show only the reliable emission, i.e. when the ratio of the fitted amplitude to the surrounding noise is larger than 3 . The gaseous kinematic maps (velocity and dispersion) discussed in this section correspond to the [O III] emission lines kinematics. The stellar $h_{3}$ and $h_{4}$ maps can be found in Fig C.2a and the $\mathrm{H} \beta$ emission line velocity and velocity dispersion maps are shown in Fig C.3a. 
In the case of the inactive galaxy NGC 4459 (Fig. C.1b), the $\mathrm{H} \beta$ line has higher signal-to-noise ratio than [O III]. $\mathrm{H} \beta$ kinematics was then used as the gaseous kinematics for this particular galaxy. Its [O III] kinematic maps are presented in Fig C.3a

Finally, NGC 4459 and NGC 6951 were observed as part of other programmes (de Zeeuw et al., 2002) with multiple fields to provide a mosaic (Fig. 3.4). For consistency with the other galaxies in our sample, we extracted images corresponding to one single SAURON field of view (FOV) from the mosaiced exposures for these two galaxies.

\subsubsection{Stellar and ionised gas distribution}

For each galaxy, the stellar continuum maps (Figs. C.1a-C.1h, first panel for each galaxy) were derived by integrating over the full wavelength window the spectra corresponding to the optimal stellar template obtained as explained in Sect. 1. We constructed the ionised gas intensity maps and the [O III]/ $\mathrm{H} \beta$ lines ratio maps directly from the fit of the emission lines spectra.

\section{Stellar continuum distribution}

Half of the galaxies in our sample present symmetric stellar continuum maps with regular isophotes. NGC 2985, 4151 (Fig. C.1e) and 4459 (Fig. C.1b) show rather round central features while the isophotes are more flattened for NGC 3227 (Fig. C.1a), 4051 (Fig. C.1e), 5806 (Fig. C.1c) and 6951 (Fig. C.1h). The latter four galaxies host a large scale stellar bar, their stellar continuum reflecting the elongation of the bar in the central regions.

Five Seyfert 2 galaxies and one inactive galaxy present more complex stellar distribution maps. Isophotal twists or irregular isophotes are observed in five Seyfert 2 galaxies: NGC 1068 (Fig. C.1a), 2655 (Fig. C.1b), 3627 (Fig. C.1c), 4579 (Fig. C.1f) and 5194 (Fig. C.1g). The inactive galaxy NGC 5055 presents an asymmetric structure (Fig. C.1g). The surface brightness is higher on the North-West side of the FOV, the emission line flux being absorbed by the dust on the South-East (see Fig. 3.4). The predominance of irregular isophotes and twists in the circumnuclear regions of Seyfert 2 galaxies compared to Seyfert 1 or inactive galaxies has been described quantitatively by Hunt \& Malkan (2004).

Finally, two inactive galaxies, NGC 3351 and 5248 exhibit a circumnuclear ring at a radius of $\sim 5^{\prime \prime}$ which corresponds to 550 pc for NGC 3351 and 200 pc for NGC 5248, respectively. These structures have been described by Devereux et al. (1992) and Laine et al. (2001). 


\section{Ionised gas distribution}

Ionised gas is detected in all the galaxies of our sample, over the full SAURON FOV except for NGC 4459 (Fig. C.1b), where emission lines are very weak outside the inner $10^{\prime \prime}$. In this Section we describe the maps of the gas properties mainly focusing on regions which are not dominated by non-gravitational motions driven by the active nucleus.

A variety of structures can be seen in the [O III] and $\mathrm{H} \beta$ intensity maps:

- Spiral-like structures are seen in two Seyfert galaxies: NGC1068 (Fig. C.1a, H $\beta$ intensity map) and NGC 4579 (Fig. C.1f). Such structures correspond well with the nuclear molecular spirals observed by Schinnerer et al. (2000b) and García-Burillo et al. (2005) in NGC 1068 and NGC 4579, respectively.

- Circumnuclear rings are found in three inactive galaxies (NGC 3351 Fig. C.1f, 5248 Fig. C.1d and 5806 Fig. C.1c) and the Seyfert 2 NGC 6951 (Fig. C.1h), which correspond with known ring-like star forming structures in the central regions of these four galaxies (Devereux et al., 1992; Laine et al., 2001; Carollo et al., 2002, respectively).

- In five other galaxies, asymmetric structures are observed. The emission line distribution of NGC 4151 is elongated from the centre to the SouthWest side of the FOV (Fig. C.1e) in agreement with the high-excitation emission line feature described by Perez et al. (1989), corresponding to the Extended Narrow Line Regions (ENLR) of this galaxy. The Seyfert 2 galaxy NGC 2655 (Fig. C.1b) presents a hot-spot 15" away from the centre to the East and a lane 10" West of the centre elongated along the South/North direction consistent with the polar ring observed by Keel \& Hummel (1988). Asymmetric and irregular structures observed in the ionised gas intensity maps of NGC 3627 (Fig. C.1c) or NGC5055 (C.1g) are certainly due to the presence of dust in the circumnuclear regions. In the case of NGC 5194 both the stellar continuum and the $\mathrm{H} \beta$ emission line intensity present irregularities consistent with the dusty nuclear spiral (Peeples \& Martini, 2006), while [O III] emission lines traces an outflow structure associated with the AGN.

- Finally three galaxies (two inactive NGC2985, Fig. C.1e, NGC4459, Fig. C.1b and the Seyfert 1 NGC 4051, Fig. C.1d) show regular round gaseous distributions.

\section{Ionised gas line ratios}

[O III] / $\mathrm{H} \beta$ ratio is a good tracer of the ionisation mechanism. Low [O III] $/ \mathrm{H} \beta$ ratios are characteristic of star formation while high $[\mathrm{O} \mathrm{III}] / \mathrm{H} \beta$ ratios trace high-ionisation processes. As expected, the Seyfert galaxies present higher 
$[\mathrm{O} I I] / \mathrm{H} \beta$ ratios than their associated inactive galaxies in the central $\mathrm{kpc}$ regions. The maximum measured ratio for the inactive galaxies is of the order of 2 , while for Seyfert galaxies it can reach values up to 10 or 20. For the Seyfert galaxies, the emission line ratio reaches its highest value in the central few arcseconds associated with the active nucleus. Some regions away from the central engine are also characterised by high $[\mathrm{O}$ III] $/ \mathrm{H} \beta$ values $(\gg 1)$ corresponding to high excitation regions, such as in the ionisation cone of NGC 1068 (Fig. C.1a), the bubble of NGC 5194 (Fig. C.1g) or the elongated gaseous feature of NGC 4151 (Fig. C.1e). Finally the ring-like structures seen in the gaseous maps of NGC 3351, NGC 5248 and NGC 5806 show low emission lines ratios ([O III $] / \mathrm{H} \beta \lesssim 0.1)$ consistent with star formation.

\subsubsection{Stellar and ionised gas kinematics}

Outside the regions dominated by non-gravitational motions associated with AGN-driven outflows, all of our galaxies show stellar and gas velocity fields with a global regular rotation pattern (second panel of each row in Figs. C.1a to C.1h). More complex dynamical structures are observed in some cases, especially in the ionised gas velocity fields.

\section{Stellar kinematics}

Most of the galaxies in our sample present a regular stellar velocity field, the orientation of their kinematic major-axis being constant over the FOV and their minor-axis perpendicular to it (e.g. NGC 2985 Fig. C.1e, 4459 Fig. C.1b, 5055 Fig. C.1g). A few active galaxies show some departures from axisymmetry e.g. the S-shaped zero-velocity line in NGC 1068 (Fig. C.1a) and twisted kinematic major-axis in NGC 3627 (Fig. C.1c). In all of our sample galaxies, the stellar kinematic major-axis is rather well aligned with the outer disc photometric major-axis (see Sect. 5.1.1). The observed stellar kinematics within the SAURON field of view are also clearly dominated, in all cases, by rotational motion, as evaluated via a global measurement of $V / \sigma$ (Dumas et al., in preparation), confirming the disc-like nature of the galaxies in our sample.

For some of our galaxies, the stellar velocity dispersion decreases inwards. These so-called $\sigma$-drops (Emsellem et al., 2001; Márquez et al., 2003) are observed in at least five Seyfert galaxies NGC 1068, 2655, 3227, 4051, 4151 and NGC 6951 and one inactive galaxy NGC5248. These central $\sigma$-drops have been found to be common in S0 to Sb spiral galaxies (Emsellem et al., 2004; Falcón-Barroso et al., 2006; Márquez et al., 2003) and recently Ganda et al. (2006) showed that such structures are present in galaxies as late as Sd. Central velocity dispersion drops are thought to be associated with dynamically cold structure, like discs and star formation regions (Wozniak et al., 2003). 


\section{Ionised gas kinematics}

The ionised gas velocity maps are dominated by rotation and for all of the galaxies the gas rotates faster than the stars (Figs. C.1a to C.1h second panels of the second rows). Since stars follow collisionless orbits, their mean velocities are indeed expected to be lower than the local circular velocity (see Binney \& Tremaine, 1987). The gas velocity fields are more distorted and present richer structures than the stellar ones. Evidence for significant deviations from axial symmetry exists in almost all the galaxies: radial change in the orientation of the major-axis (NGC 3227, Fig. C.1a), wiggles along the kinematic minor-axis (NGC 2655, Fig. C.1b , 4579, Fig. C.1f), S-shaped feature (NGC1068, Fig. C.1a), or more complicated structures (e.g. NGC3627, Fig. C.1c or NGC 5194, Fig. C.1g ). For all of our galaxies, the $\mathrm{H} \beta$ and [O III] velocity fields present very similar structure, though [O III] velocity fields have generally better signal-to-noise ratio than the $\mathrm{H} \beta$ ones, except for NGC 4459 (Figs. C.1a and C.3a). We also observe highly misaligned kinematic majoraxes of the ionised gas with respect to the stellar ones in some of our galaxies such as NGC 2655 (Fig. C.1b) and NGC 4579 (Fig. C.1f). These misalignments will be analysed in the following Section.

The gaseous velocity dispersion maps present no particular structure for all of the inactive galaxies and two Seyferts (NGC 3627, Fig. C.1c and 4051, Fig. C.1d). For eight Seyfert galaxies (NGC 1068, 2655, 3227, 3627, 4151, 4579, 5194 and 6951) and three inactive galaxies (NGC 2985, 4459 and 5055) $\sigma$ values increase inwards. In the inner $5^{\prime \prime}$, the dispersion can reach values 200 $\mathrm{km} \mathrm{s}^{-1}$ higher than in the outer part of the FOV as in NGC1068 (Fig. C.1a). NGC 2655 (Fig. C.1b) and 4579 (Fig. C.1f) also present high dispersion values $\left(\sim 230 \mathrm{~km} \mathrm{~s}^{-1}\right)$ associated with the gas distribution structures. For NGC 4151, gaseous dispersion velocity maps exhibit high values in the inner arcseconds (about $280 \mathrm{~km} \mathrm{~s}^{-1}$ ). Outside the very inner part and inside the ENLR, the velocity dispersion shows lower values (between 50 and $130 \mathrm{~km} \mathrm{~s}^{-1}$ ). Then, $\sigma$ increases in the outside parts of the map for this galaxy, corresponding to the location of a dusty ring (Vila-Vilaro et al., 1995).

\subsection{H I moment maps}

As explained in Sec 3.2.1, radio data are available for 50 of the 56 galaxies of the VHIKINGS survey, via archival data or dedicated VLA observations. For two of the observed galaxies, NGC 4459 and NGC 7743, neutral hydrogen has not been detected. Regarding NGC 1068, the moment maps are contaminated by strong $\mathrm{HI}$ absorption from the nucleus and were then discarded. Overall $\mathrm{HI}$ data of 47 galaxies of the VHIKINGS sample are available. The data reduction and construction of the moment maps of these galaxies are on-going: in this section I present the H I distribution (zeroth moment, Sec. 4.2.1) and velocity field (first moment, Sec. 4.2.2) for the 21 galaxies for which data reduction has 
already been completed. The detailed analysis of the H I moment maps (e.g. $\mathrm{H}$ I mass and extent) and environment (distribution of low surface brightness companions) for the galaxies of the VHIKINGS sample and the connection of their H I properties and their activity state, will be found in Mundell et al. (in prep) and Haan et al (in prep).

\subsubsection{H I morphology and environment}

Figs. D.1a to D.1f (Appendix D) present the H I first two moment maps of the Seyfert and non active galaxies of the VHIKINGS sample for which data reduction has been completed so far. The optical DSS image is also shown for comparison, on the left for each galaxy. The $\mathrm{H}$ I intensity distribution is shown in the middle panel, and the H I velocity field on the right. The extent of the neutral hydrogen is much larger than the optical image for the majority of the galaxies presented here (e.g. NGC 2655, 2985, 5055 and 5383). Near the centre there is generally a depression in the H I distribution (e.g. NGC3351, 4051 and 4548). Some galaxies present companions (NGC 2655, 3169, 3227, $3963,4041,4593,4639$ and 5383) and tidal or interaction structures are seen for these galaxies: elongated structure towards the companion (NGC 3169, 3227, 3963) or loops (NGC 2655, 4593). In the case of NGC 3227, no H I emission is associated with the companion NGC 3226 and the southern plum is much more elongated and narrower than the plum on the North pointing towards NGC 3226. These two features are associated with the interaction between these two galaxies (Mundell et al., 1995b). H I is not detected in the central regions, corresponding the optical emission, in NGC 5395. This galaxy presents an outer H I ring, broken in the southern part. For NGC 4151, the central H I distribution traces the large scale stellar bar. Then outer H s spiral arms are seen (Mundell et al., 1999; Mundell \& Shone, 1999). These spiral arms are very weak in the optical counterpart, as shown in the SDSS image on Fig. 4.2.1. The majority of galaxies without any companion shows regular $\mathrm{H}$ I distribution, tracing the stellar structures such as spiral arms (e.g NGC 4051, 6951) or bars (e.g. NGC 5383) in the central regions and with usually an H I outer disk (e.g. NGC 5383, 6951). Finally, some galaxies present an outer warp in the H I distribution, e.g. NGC 3705 or 4258.

\subsubsection{HI kinematics}

The H I velocity fields are shown in Figs. D.1a to D.1f. All the galaxies presented here show H I velocity field dominated by global rotation. Some deviations to pure rotational motions are seen though. Some galaxies present irregular isovelocity contours, with twists or wiggles (e.g. NGC 2655, 4941, 6951) and in some cases the orientation of the kinematics major axis changes with a great amplitude as in the case of NGC 3169 or NGC 2967 for which the central arcminute seems almost decoupled from the outer parts. 


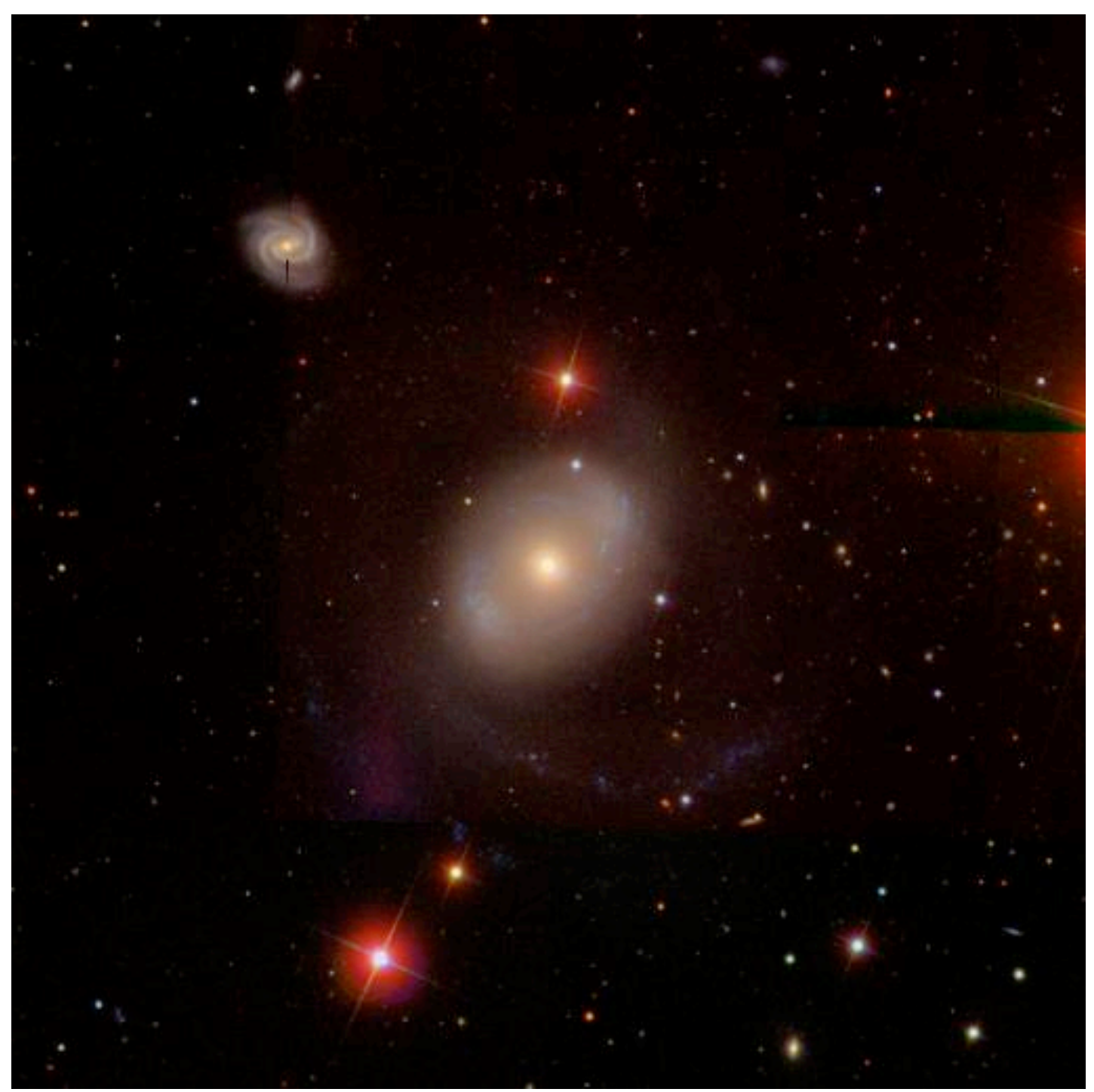

Figure 4.1: SDSS image of NGC 4151. The outer spiral arms are visible though very weak. 
The H I velocity fields of these galaxies will be analysed and discussed in details, along with the optical stellar and gaseous velocity fields, in Chapter 5.

\subsection{Summary}

In this chapter, the moment maps of our galaxies were presented for the stellar, ionised gas and $\mathrm{H}$ I components. In the circumnuclear regions, the stellar kinematics present very regular rotation patterns for both Seyfert and nonactive galaxies in agreement with previous studies (Barbosa et al., 2006). The ionised gas is generally o-rotating with respect to the stars. The gaseous kinematic maps in the circumnuclear regions reveal some irregular and complex features. These perturbations in the gaseous velocity fields may hint for the presence of streaming or radial flows, which could be related to the fuelling mechanisms. This will be further examined in the next chapter. 


\section{Chapter 5}

\section{Kinematic analysis of the velocity fields}

In the previous Chapter, we described the maps of the stellar, ionised gas and neutral gas components. For all of our galaxies, the stellar velocity fields are dominated by rotation, showing regular isovelocity contours and their photometric and kinematic major-axes seem globally aligned. The neutral gas kinematics show regular isovelocity contours as well, in agreement with global rotation motions. In comparison with the stellar and $\mathrm{H}$ I maps, however, the gaseous distributions are more complex and the kinematics more perturbed with this effect appearing more pronounced for the Seyferts compared to their control galaxies. The identification of this qualitative difference between gaseous and stellar distributions and kinematics in the inner kpc of active and inactive galaxies has important implications for the triggering and nuclear fuelling. In this Chapter, we therefore provide a more quantitative analysis of the velocity fields of the stars, ionised and neutral gas. We first compare the major-axis orientation of the stellar velocity field in the central regions to that of the outer galactic disk. Then we quantify the differences between stars and gas in Seyfert and inactive galaxies as seen in our SAURON maps. We compare the global orientation of the stellar and ionised gas kinematic maps to probe any significant differences between the two components. Then we analyse the kinematic maps in the central regions (stars and ionised gas) and outer disk (neutral gas) in more detail, by computing the kinematic parameters (systemic velocity, dynamical centre, PA, rotational velocity) as a function of radius, using a simple representation of a two-dimensional thin disk in rotation.

\section{1 global kinematic major-axis orientation in the central regions}

The stellar and ionised gas velocity fields can be first simply parametrised by their major-axes. Misalignments between the kinematic major-axes of the stel- 

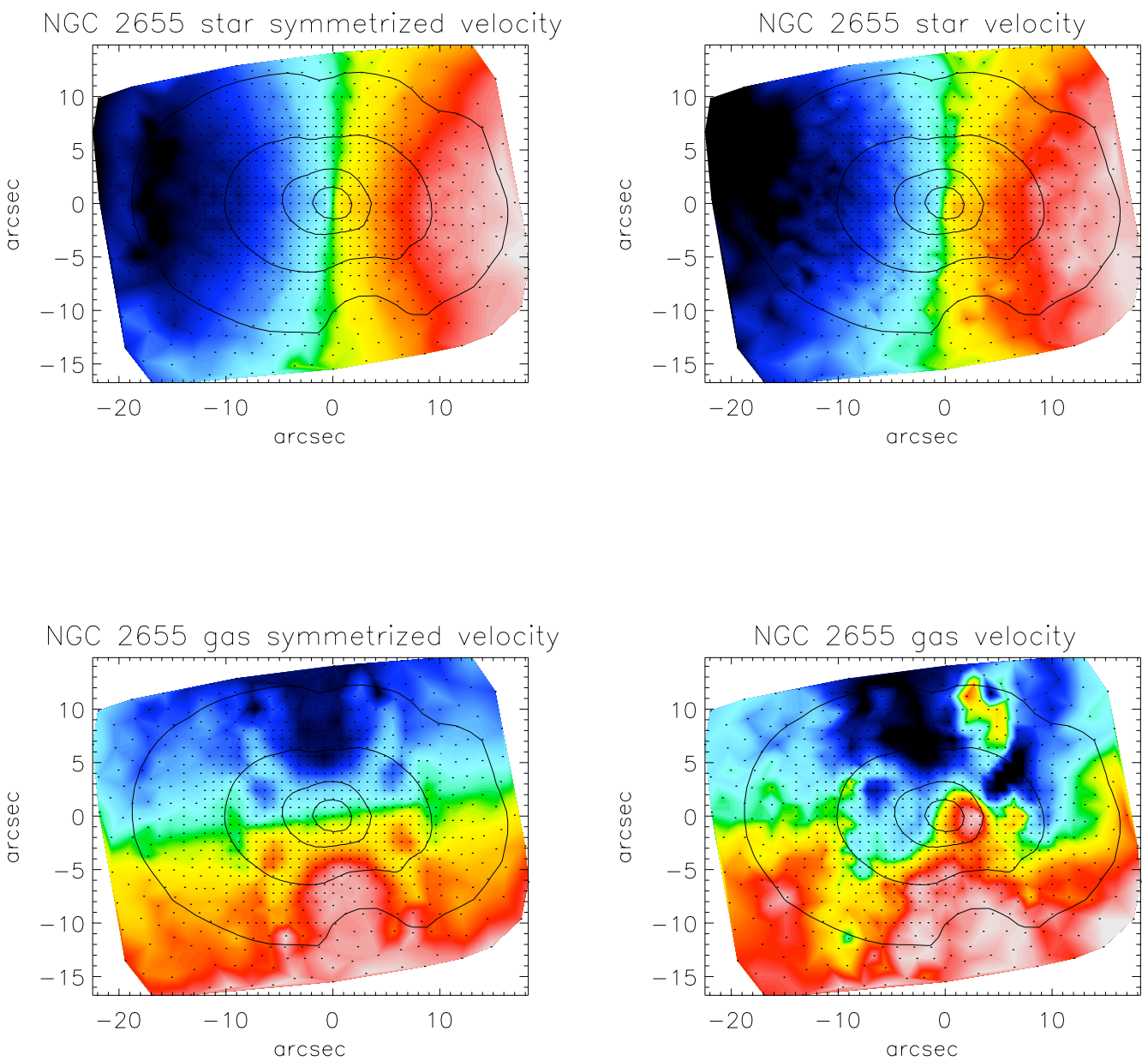

Figure 5.1: Comparison of stellar and gaseous observed (right panels) and biantisymmetric (left panels) velocity fields for NGC 2655, at the best fitting PA. Contours correspond to the stellar continuum. 
lar and ionised gas components may reveal the presence of non-axisymmetric structures or decoupled components such as central disks. Here, we compare such misalignments in our sample of Seyfert and control galaxies, to characterise global kinematic differences in their central kpc.

The kinematic axis orientations were determined for both the stellar and ionised gas velocity fields. The global position angles (PAs) of the kinematic major-axis were obtained by minimising the differences between our SAURON velocity fields (stars and [O III]) and a bi-antisymmetric representation of these fields. Such bi-antisymmetric velocities $V^{\prime}(x, y)$ correspond to the average of the velocities in the four quadrants defined by the major and minor kinematic axes:

$$
V^{\prime}(x, y)=\frac{V(x, y)+V(x,-y)-V(-x, y)-V(-x,-y)}{4}
$$

where the $\mathrm{x}$-axis and $\mathrm{y}$-axis are along the major and minor axes, respectively. This method is described in more details by Krajnović et al. (2006) in their Appendix $C$ and we used a specific implementation written by Michele Cappellari. Fig. 5.1 shows the stellar and gaseous velocity fields for NGC 2655 (right panels) and their corresponding bi-antisymmetric velocity maps (right panels).

We applied this method to the SAURON maps described in Chap. 4. In order to compute the kinematics PA due to motions in the gravitational field of the host galaxy, regions contaminated by the AGN were masked out, in the case of the Seyfert galaxies. The [O III]/ $\mathrm{H} \beta$ line ratio was used to determined these regions, following criteria defined by Ho et al. (1997): regions for which [O III] $/ \mathrm{H} \beta>3$ are considered typical of photoionization regions from the AGN, and then were masked out to compute the kinematic PA. In the case of NGC 4151, the ENLR is very extended, and [O III] $/ \mathrm{H} \beta>3$ in $95 \%$ of the FOV. Therefore for this galaxy, we masked out regions of line ratio [O III]/ $\mathrm{H} \beta>7$. The values for the measured kinematics PAs and misalignments between the stars and ionised gas are listed in Table 5.1.

\subsubsection{Stellar kinematics and the orientations of the line of nodes}

In the case of an axisymmetric mass distribution, the direction of the stellar kinematic major-axis should coincide with the line-of-nodes (LON) of the galaxy, while in the case of a triaxial potential the kinematic and photometric major-axes can depart from each other due to projection effects. Therefore, we first check if the stellar kinematic major-axis in the central regions and the outer photometric major-axis are aligned. The photometric major-axis position angle is computed from ellipse fitting on the R-band DSS images for each of our galaxies. These derived values are then compared with published ones. For most of our galaxies, the PAs values found in the literature are in good agreement with those derived from the ellipse fit, within the error bars, in which case we took our measured values as the photometric PAs. How- 


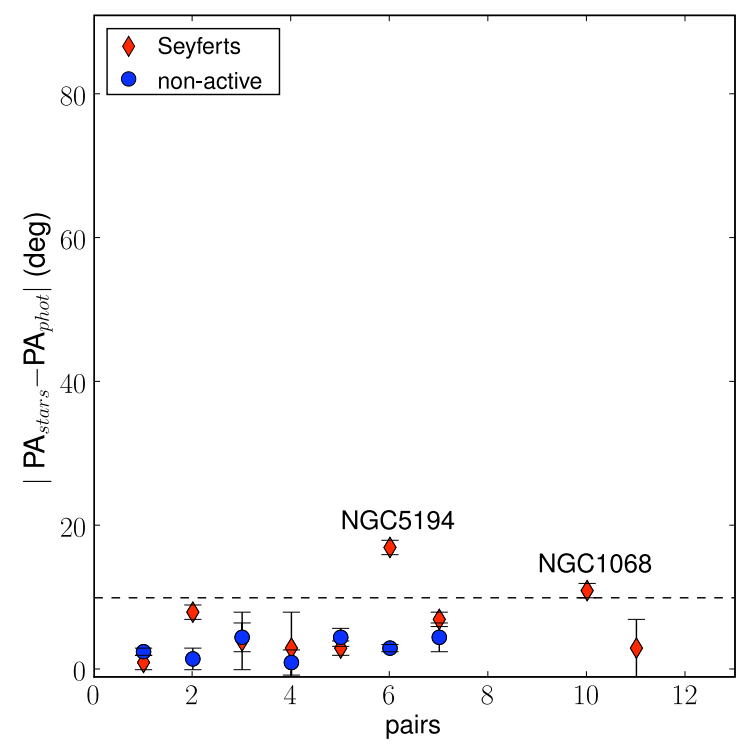

Figure 5.2: Distribution of the differences between the photometric and the global stellar kinematics PAs $P A_{\text {stars }}-P A_{\text {phot }}$ for each pair of galaxies, Seyferts (red diamonds) and inactive galaxy (blue circles). The dashed black horizontal line corresponds to a difference of $10^{\circ}$.

ever, a few galaxies present significant discrepancies between our fitted values and the published ones. This is the case for NGC 1068 and NGC 4151 which present a weak oval bar and a faint, almost circular outer disk, respectively. The measurement of the photometric PA of NGC5194 is uncertain given that this galaxy is nearly face-on $\left(\mathrm{i}=20^{\circ}\right)$. We therefore used reliable values derived from HI kinematics for NGC 4151 (Pedlar et al., 1992) and optical kinematics for NGC 5194 (Tully, 1974), as the photometric PAs. Table 5.1 lists the resultant photometric PA values for the sample galaxies. The differences between the global stellar kinematic and the photometric PAs are plotted in Fig. 5.2.

Our first qualitative impression is confirmed: the stellar kinematic PAs are parallel to the photometric ones within $10^{\circ}$ for all of our galaxies except for NGC 5194 and NGC 1068 which present a difference of about $25^{\circ}$ and $12^{\circ}$, respectively. In the case of NGC 1068, the stellar kinematic major-axis PA corresponds to the average PA of the kinematic axis as fitted on the large-scale HI data by Bland-Hawthorn et al. (1997), while the outer photometric major-axis lies at a PA of about $80^{\circ}$ (Emsellem et al., 2006). Besides the two specific cases of NGC 5194 and NGC 1068, for all of our galaxies the major-axis orientation of the central part of the stellar velocity field is therefore a reliable measurement of the line-of-nodes of the galaxy. 


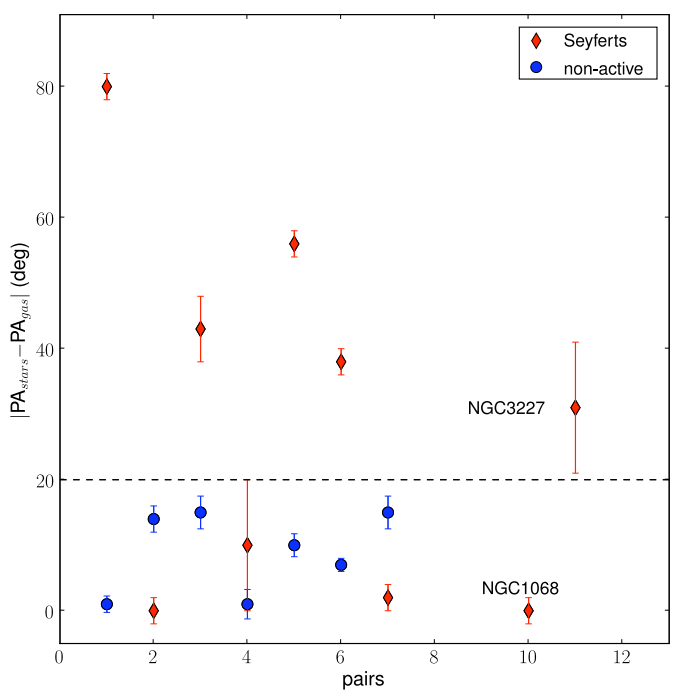

Figure 5.3: Distribution of differences of global kinematics PAs of stars and gas $P A_{\text {stars }}-P A_{\text {gas }}$ for each pair of galaxies, Seyfert (red diamonds) and inactive galaxies (blue circles). The dashed black horizontal line corresponds to a kinematic misalignment of $20^{\circ}$.

\subsubsection{Global kinematic misalignments between stars and ionised gas}

Fig. 5.3 presents the global gaseous versus stellar kinematic PAs and the distribution of kinematic misalignments between the stellar and gaseous components in our sample. All the inactive galaxies present a misalignment between gaseous and stellar kinematic major-axes less than $20^{\circ}$ while five Seyfert galaxies show an absolute misalignment larger than $20^{\circ}$. A horizontal line at a kinematic misalignment of $20^{\circ}$ is drawn in the Fig. 5.3 to enhance this result. This arbitrary value of $20^{\circ}$ has been chosen since it is a maximum limit of misalignments for all the non-active galaxies, while some Seyfert galaxies present higher kinematic misalignments. Among the five Seyfert galaxies with strong kinematic misalignments, two are Seyfert 1 galaxies: NGC 3227 and NGC 4051 (pair 3) for which the BLR is detected and one is NGC 5194 (pair 6) which presents an outflow structure in the gaseous maps on the Northern side (Fig C.1g). The other two galaxies are NGC 2655 (pair 1) and NGC 4579 (pair 5). NGC 2655 is known to host off-planar gas in the central regions (Erwin \& Sparke, 2003). An inner molecular spiral is present in the nuclear region of NGC 4579 (García-Burillo et al., 2005) and a spiral-like structure is observed in our ionised gas distribution maps (Fig. C.1f). 


\subsubsection{Statistical tests}

About half of our Seyferts sample presents strong misalignments between stellar and gaseous kinematics while none of the inactive galaxies shows such differences. In order to determine whether the two distributions are statisticallysignificant different, we applied statistical tests. Since NGC 1068 and 3227 do not have associated control non-active galaxies, we only take the 7 pairs of active/inactive galaxies into consideration. Therefore we considered samples of 7 Seyfert galaxies and 6 inactive galaxies. For such small samples, only nonparametric statistical tests can be applied and we then used two well suited tests: the Kolmogorov-Smirnov test and the Mann-Whitney U test. In the following, I will describe these two tests and their results on the kinematic misalignments distributions. For both of these tests, our null-hypothesis $H_{0}$ is that the kinematic misalignments of the Seyfert and inactive galaxies come from the same distribution, while our alternative hypothesis $H_{1}$ would be that the two distributions are different (two-tailed test). We will consider that the two populations are statistically different, and hence reject $H_{0}$, if the significance level is $5 \%$ or less. At this level of significance, the probability that the two populations are different would be of $95 \%$ or more.

\section{- The Kolmogorov-Smirnov test.}

The Kolmogorov-Smirnov test (KS test) is a powerful non-parametric test. It considers the maximum deviation $\mathrm{D}$ between the cumulative distribution functions of the two samples. The cumulative distribution function, $Z$, describes the probability that a variable $X$ takes on a value less than or equal to a number $x$. In our case, it is defined as the numbers of galaxies for which $\left|\mathrm{PA}_{\text {stars }}-\mathrm{PA}_{\text {gas }}\right|=\Delta \mathrm{PA}$ is less than a considered value, divided by the total number of galaxies in the sample. Fig. 5.4 presents the cumulative distribution functions of the Seyfert (red curve) and non-active galaxies (blue dashed curve) populations.

The maximum deviation between these two functions is $\mathrm{D}=0.57$. The critical values of $\mathrm{D}$ for our sample sizes at a significance level of $5 \%$ is $\mathrm{D}_{c}$ $=0.714$. Therefore $\mathrm{D}<\mathrm{D}_{c}$ and the null-hypothesis cannot be rejected at the chosen degree of significance.

\section{- The Mann-Whitney U test}

The KS test is a very powerful statistic test, however, for samples of small sizes like ours, the error to accept a false null-hypothesis may occur. Since we cannot reject $H_{0}$ via the KS test on our data, we run another statistical test, to check whether the first statistical result is due to our small samples sizes. We then used the Mann-Whitney test, or U-test. This test considers the ranks of the values of the variables ( $\triangle \mathrm{PA}$ in our case) for the two populations. The two samples are combined and sorted. Then, taking each value of $\triangle \mathrm{PA}$ in the sample of inactive galaxies, we count the number of $\triangle \mathrm{PA}$ of Seyfert galaxies that are smaller than it, and we count a half for any that are equal to it. The statistic variable $U$ is finally 


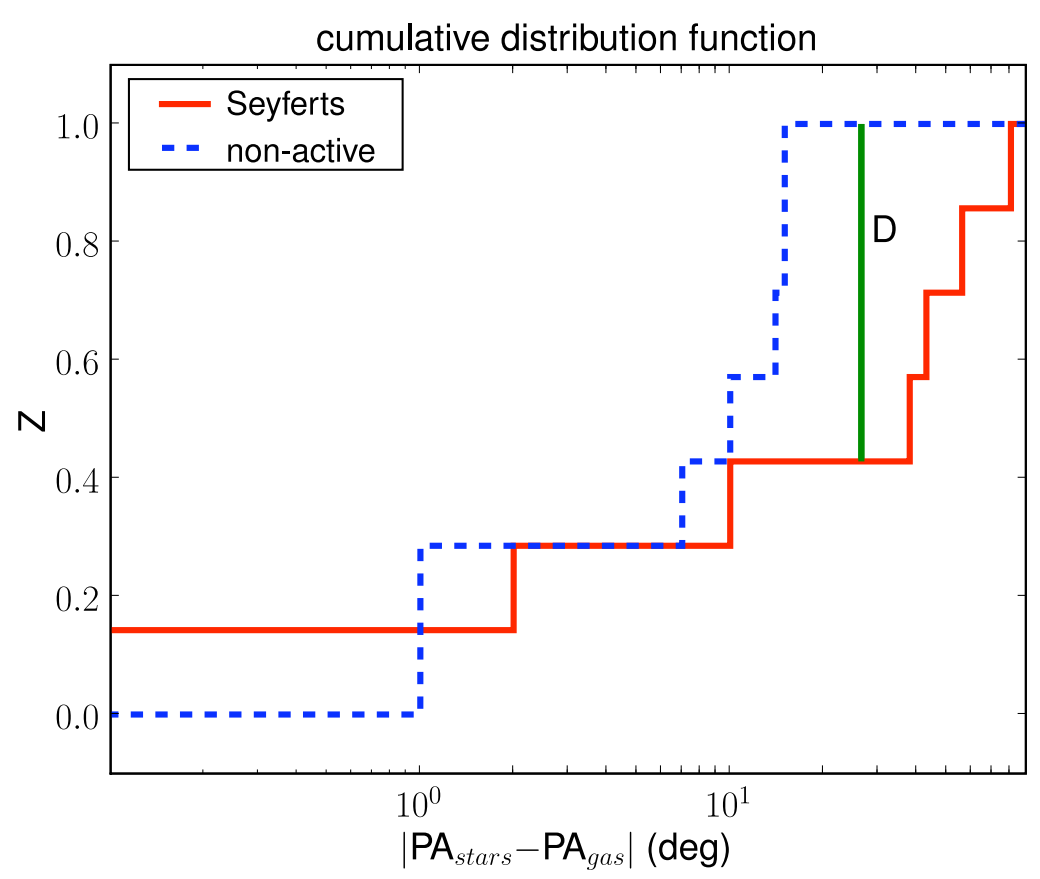

Figure 5.4: Cumulative distribution functions of the Seyfert (red solid curve) and the non-active galaxies (blue dashed curve). The KS statistic D is shown as the green line. A log scale has been used to enhance the difference between the two distributions.

computed by summing all these counts. In the case of totally separated sample, e.g. $\triangle \mathrm{PA}$ of Seyfert galaxies always larger than those of nonactive galaxies, the statistic $U$ for the non-active galaxies sample would be 0 while $U$ of the Seyfert galaxies would be 42 (e.g. the product of the sample sizes). For small size samples, critical values of $U$ are tabulated, at the considered level of significance. The null-hypothesis can be rejected if the value of $U$, computed from our data is less than the corresponding critical value. In the case of our sample size, the critical value is $\mathrm{U}_{c}=8$ at a significance level of $5 \%$, while the computed value is $\mathrm{U}=$ 13 for the non-active galaxies sample (for the Seyfert galaxies, $\mathrm{U}=28.5$ ). Therefore, the distributions of the kinematic misalignments of two populations are the same at our chosen degree of significance. This result is in good agreement with the KS test. However, the U-test shows that the null-hypothesis can be rejected at a degree of significance of $1.5 \sigma \sim 15 \%$. The fact that $H_{0}$ cannot be rejected with a higher probability is certainly due to the small sizes of our samples.

\section{- Results}

Finally, no statistically-significant difference is found between Seyfert and control-galaxies at a level of significance of 5\%. The Mann-Whitney $\mathrm{U}$ test showed however that the two distributions can be considered different with a probability of about $85 \%$, which shows that the Seyfert galaxies tend to present higher kinematic misalignments than the 
non-active galaxies in our samples.

In this section, we symmetrized the velocity fields in order to look for global differences in disk orientation. This zeroth order analysis shows that half of the Seyfert galaxies present kinematic misalignments between the ionised gas and the stars larger than $38^{\circ}$ while such misalignments are less than $20^{\circ}$ in the case of all the non-active galaxies. However, our samples are too small to be able to statistically separate the two distributions of active and inactive galaxies in term of kinematic misalignments.

\subsection{Modelling the velocity fields}

In the above analysis, global parameters (independent of radius) were extracted from the two-dimensional SAURON data, and therefore details of the observed kinematic structures and small-scales variations of the PA are not accounted for. Analysis of the radial variations of the kinematic parameters of the galaxies is essential to study the galactic dynamical structure, and allows therefore to constrain any dynamical processes which may be responsible for the transport of material in the disk of galaxies. The velocity curves of galaxies, which give the circular orbital velocity as a function of radius, were first extracted from long-slit spectroscopic observations. In this case however, the kinematic information is only available along the direction of the slit, and misalignment of the slit with respect to the major-axis or deviations from pure circular motions may change the shape of the derived rotation curve and thus the results of such analysis may be difficult to interpret (Whitmore et al., 1988).

The analysis of two-dimensional velocity maps of galactic disks, obtained with a variety of instruments (Radio and Fabry-Perot interferometers, IFS) provides much more kinematic information. It allows to derive more robustly the velocity curve of the galaxies, and also to reconstruct a projected velocity field model, corresponding to purely circular orbits. The subtraction of this model from the observed velocity map gives the residual velocity field. Assuming that the model corresponds to the circular components, these residual velocities correspond then to the non-circular motions, and careful analysis of such residual velocity maps allows to constrain the dynamics of the galactic disk. Many methods were developed in order to robustly determine the galactic kinematic parameters from two-dimensional velocity fields. In the well used 'tilted-ring' method (Rogstad et al., 1974), motions in galactic disks are supposed to be dominated by circular rotation, with potentially small non-circular components. The galactic disk is divided into concentric rings. The figure 5.5 presents the stellar velocity fields of NGC 5055, divided in 15 concentric rings of width of $1^{\prime \prime} .5$ running from $2^{\prime \prime} .75$ to $23^{\prime \prime} .75$. Each rings is defined by the angles $i$ and $P A$ and are centred on the dynamical centre. The kinematic and geometrical parameters are then assumed to be constant within a ring and are 


\begin{tabular}{|l|l|l|c|c|c|c|c|c|}
\hline Pairs & $\begin{array}{l}\text { Name } \\
\text { NGC }\end{array}$ & Activity & $\begin{array}{c}\text { PA }_{\text {phot }} \\
(\mathrm{deg}) \\
(1)\end{array}$ & $\begin{array}{l}\text { Ref } \\
(2)\end{array}$ & $\begin{array}{c}\mathrm{PA}_{\text {stars }} \\
(\mathrm{deg}) \\
(3)\end{array}$ & $\begin{array}{c}\mathrm{PA}_{\text {gas }} \\
(\mathrm{deg}) \\
(7)\end{array}$ & $\begin{array}{c}\Delta \mathrm{PA}_{\text {phot-kin }} \\
(\mathrm{deg}) \\
(8)\end{array}$ & $\begin{array}{c}\Delta \mathrm{PA}_{\text {kin }} \\
(\mathrm{deg}) \\
(9)\end{array}$ \\
\hline \multirow{2}{*}{1} & 1068 & S2 & $-100 \pm 5$ & 1 & $-89 \pm 1$ & $-89 \pm 1$ & $11 \pm 6$ & $0 \pm 2$ \\
\cline { 2 - 9 } & 3227 & S1 & $158 \pm 2$ & 2 & $161 \pm 4$ & $192 \pm 6$ & $3 \pm 6$ & $31 \pm 10$ \\
\hline 2 & 2655 & S2 & $-95 \pm 5$ & 3 & $-94 \pm 1$ & $-174 \pm 1$ & $1 \pm 6$ & $80 \pm 2$ \\
& 4459 & inactive & $-77 \pm 2$ & 3 & $-80 \pm 1$ & $-81 \pm 1$ & $3 \pm 3$ & $1 \pm 2$ \\
\hline 3 & 3627 & S2 & $-175 \pm 5$ & 3 & $-167 \pm 1$ & $-167 \pm 1$ & $8 \pm 6$ & $0 \pm 2$ \\
& 5806 & inactive & $172 \pm 2$ & 3 & $171 \pm 2$ & $157 \pm 1$ & $1 \pm 4$ & $14 \pm 3$ \\
\hline 4 & 4051 & S1 & -45 & 4 & $-49 \pm 4$ & $-6 \pm 1$ & $4 \pm 4$ & $43 \pm 9$ \\
& 5248 & inactive & $110 \pm 2$ & 3 & $115 \pm 2$ & $130 \pm 1$ & $5 \pm 4$ & $15 \pm 3$ \\
\hline 5 & 4151 & S1 & 22 & 5 & $25 \pm 5$ & $34 \pm 5$ & $3 \pm 5$ & $9 \pm 10$ \\
& 2985 & inactive & $-3 \pm 1$ & 3 & $-2 \pm 2$ & $-3 \pm 1$ & $1 \pm 3$ & $1 \pm 3$ \\
\hline 6 & 3579 & S2 & $96 \pm 2$ & 3 & $99 \pm 1$ & $155 \pm 1$ & $3 \pm 5$ & $56 \pm 2$ \\
& 3351 & inactive & $-168 \pm 2$ & 3 & $-164 \pm 1$ & $-154 \pm 1$ & $6 \pm 3$ & $10 \pm 2$ \\
\hline 7 & 5194 & S2 & -190 & 6 & $-173 \pm 1$ & $-135 \pm 1$ & $17 \pm 1$ & $38 \pm 2$ \\
& 5055 & inactive & $102 \pm 2$ & 3 & $99 \pm 1$ & $106 \pm 1$ & $3 \pm 4$ & $7 \pm 2$ \\
\hline
\end{tabular}

Table 5.1: Results of the kinematic analysis of the stellar and gaseous components in the central regions. (1): Pair identifier; (2): NGC number; (3): Activity; (4): Photometric position angle (PA) in degrees. The values for NGC 4151 and NGC 5194 correspond to the line of nodes PA. (5): Origin of the values for the photometric PA: 1: Emsellem et al. (2006), 2: Mundell et al. (1995b), 3: R-Band DSS images ellipse fitting, 4: Christopoulou et al. (1997), 5: Pedlar et al. (1992), 6: Tully (1974), 7: Rozas et al. (2002); (6): Stellar Kinematic position angle (PA), in degrees; (7): Gas Kinematic position angle (PA), in degrees; (8): Difference between the photometric and the stellar kinematic PAs, in degrees $\Delta \mathrm{PA}_{\text {phot-kin }}=\left|\mathrm{PA}_{\text {phot }}-\mathrm{PA}_{\text {stars }}\right| ;$; (9): Difference between the stars and gas kinematic $\mathrm{PAs}$, in degrees $\Delta \mathrm{PA}_{\text {kin }}=\left|\mathrm{PA}_{\text {stars }}-\mathrm{PA}_{\text {gas }}\right|$. 


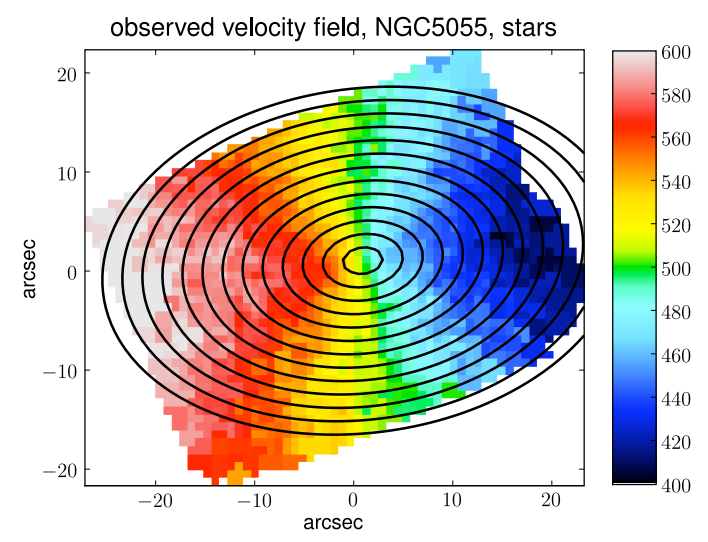

Figure 5.5: NGC 5055 stellar velocity field divided in concentric rings. The width of each ring is $1^{\prime \prime} .5$, and the mean radii run from $2^{\prime \prime} .75$ to $23^{\prime \prime} .75$.

then fitted. Such 'tilted-ring' fit have been implemented in several data analysis softwares, such as the ROTCUR (Begeman, 1987) routine in GIPSY (Groningen Image Processing System) and its extension RESWRI (Schoenmakers et al., 1997).

Considering an infinitesimally thin two-dimensional galactic disk, therefore with no vertical velocities, the line-of-sight (LOS) velocity can be written as a function of the polar coordinates in the galactic disk $(R, \Phi)$ and the disk inclination $i$, in the most general case:

$$
\begin{aligned}
V_{\text {los }}(R, \Phi, i)=V_{\text {sys }} & +V_{\Phi}(R, \Phi) \cos \Phi \sin i \\
& +V_{R}(R, \Phi) \sin \Phi \sin i
\end{aligned}
$$

where $V_{\text {sys }}$ is the systemic velocity of the galaxy and $\left(V_{R}(R, \Phi), V_{\Phi}(R, \Phi)\right)$ is the velocity vector in the plane of the galaxy. The disk inclination $i$ ranges from $0^{\circ}$ (face-on) to $90^{\circ}$ (edge-on). Finally, the polar coordinates in the plane of the galaxy $(R, \Phi)$ are related to the observable coordinates $(x, y)$ on the sky plane by:

$$
\left\{\begin{array}{l}
\cos \Phi=\frac{-\left(x-x_{c}\right) \sin \Phi_{0}+\left(y-y_{c}\right) \cos \Phi_{0}}{R} \\
\sin \Phi=\frac{-\left(x-x_{c}\right) \cos \Phi_{0}-\left(y-y_{c}\right) \sin \Phi_{0}}{R \cos i}
\end{array}\right.
$$

$\left(x_{c}, y_{c}\right)$ are the coordinates of the dynamic centre and $\Phi_{0}$ is the PA of the projected major-axis of the disk measured with respect to North (counterclockwise). The angle $\Phi$ is defined from the x-axis as shown in the bottom panel of Fig. 5.6. This figure presents the projection of the galactic disk on the sky plane (top left panel). The x-axis of the galactic plane is chosen so that it is common to the galactic and sky plane. The projection on the sky is then done 

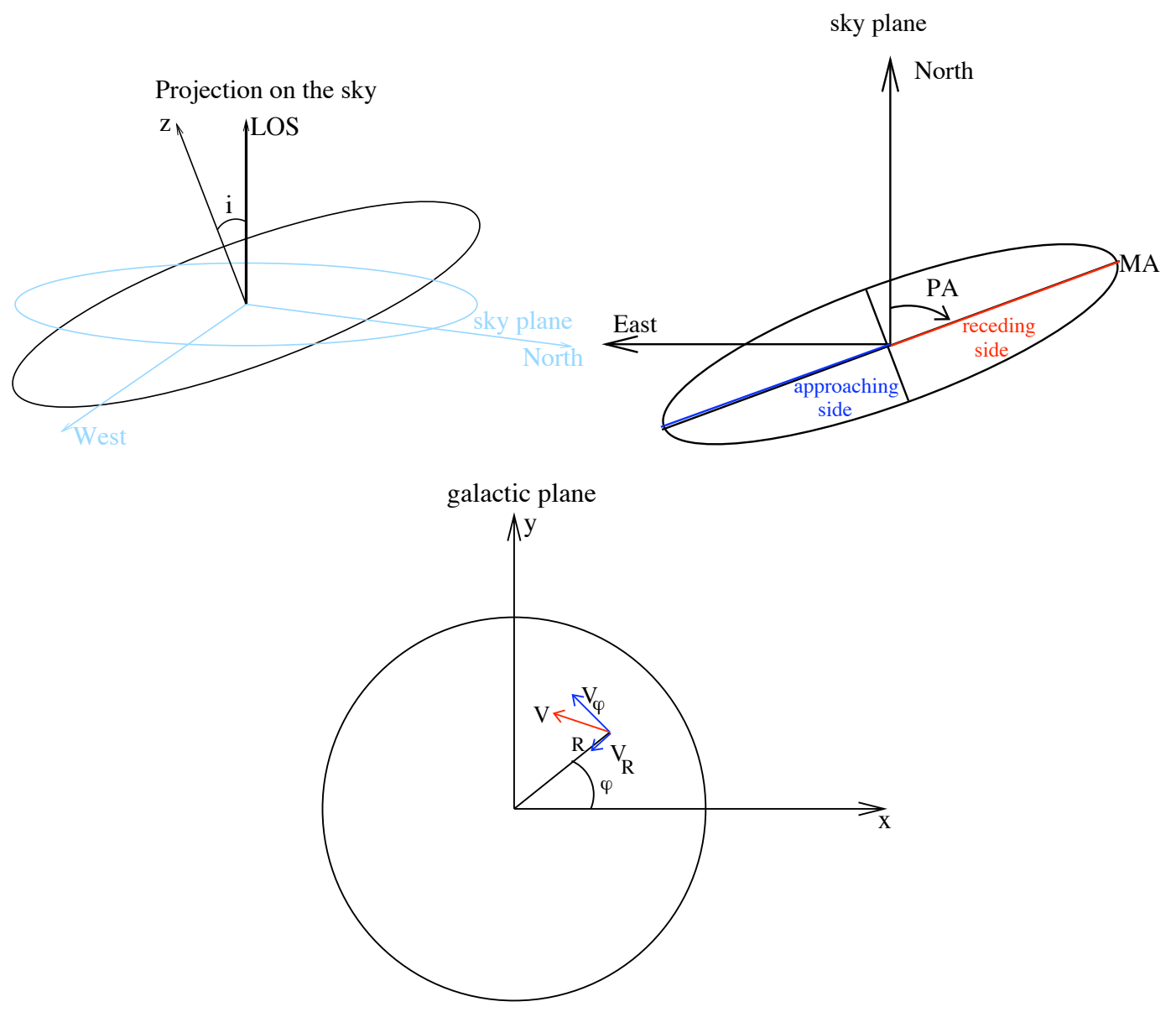

Figure 5.6: Projection of the galactic disk on the sky. Top left: view of the projection of the galactic disk on the sky plane (light blue ellipse and axes). The $z$-axis is the axis perpendicular to the galactic plane. The disk inclination $i$ is the angle between the z-axis and the line-of-sight (LOS). Top right: View of the projected galactic disk. MA corresponds to the major-axis of the ellipse. The position angle (PA) of the galaxy is defined from the North to the majoraxis on the receding side (red line), counter-clockwise. In this figure PA is therefore negative. The blue line corresponds to the approaching side. Then, the bottom figure shows the velocity vector $\mathrm{V}$ decomposed as $V_{R}$ and $V_{\Phi}$ at the position $(R, \Phi)$ in the galactic plane. 
via a rotation of angle $\mathrm{i}$ around the $\mathrm{x}$-axis. Therefore, the disk inclination does not take place in the expression of the $\cos \Phi$ term.

In the simplest case of pure rotation motions in an axisymmetric potential with rotation velocity $V_{c}$, Eq. 5.1 becomes:

$$
V_{\text {los }}=V_{\text {sys }}+V_{c}(R) \cos \Phi \sin i
$$

However, this simple modelling does not take into account the complex non circular motions that occur in most observed velocity fields. As shown in the stellar and ionised gas velocity fields of a number of Seyfert and non active galaxies (Chap. 4), complex kinematic structures are observed. The kinematic PA may change as a function of radius, isovelocity contours are not regular and the zero-velocity line (kinematic minor-axis) can be twisted. Therefore, many studies have been made to investigate these complex kinematic structures (Schoenmakers et al., 1997; Wong et al., 2004). Following these previous works, we then express the LOS velocity fields in terms of a Fourier series:

$$
V_{\text {los }}=c_{0}+\sum_{n=1}^{k}\left[c_{n}(R) \cos (n \Phi)+s_{n}(R) \sin (n \Phi)\right],
$$

where $k$ is the number of harmonics, $c_{n}$ and $s_{n}$ are the harmonic coefficients, depending only on $\mathrm{R}$ and which contain the kinematic information. Hence, $c_{0}$ is immediately related to $V_{\text {sys }}$ as $c_{0}=V_{\text {sys }}$ and in the simple case of pure circular rotation motions, $c_{1}=V_{c} \sin i$ and all the other coefficients are zero. For any observed velocity field, the different harmonic coefficients are connected to the kinematic parameters and hence to the gravitational potential and the dynamical perturbations, sources of observed non-circular motions. Extension of the simple tilted-ring model were implemented to take into account non-circular motions and to perform harmonic expansion of the velocities along the rings, such as the GIPSY routine RESWRI (Schoenmakers et al., 1997). These first works were dedicated to the analysis of radio interferometric data. More recently, routines were developed to perform tilted-ring fits on velocity fields obtained with optical IFS, such as for the SAURON data. Fathi et al. (2005) analysed the SAURON velocity field of NGC 5448 via harmonic decomposition, and modelled the underlying gravitational potential using the harmonic coefficients. The Kinemetry tool developed by Krajnović et al. (2006) perform high order harmonic analysis of two-dimensional velocity maps without any assumption on the structure of the observed system (e.g. motions confined in a thin disk).

In this work, we have the full two-dimensional dynamical information. We can then analyse the stellar and ionised gas and neutral gas velocity fields quantitatively over the FOV and study the kinematic properties as a function of radius, using such tools . Our radio imaging resolution gives access to the $\mathrm{H}$ I disk dynamics down to about $20^{\prime \prime}$ (i.e. $\mathrm{r} \approx 700 \mathrm{pc}$ for the VHIKINGS sample). Then the inner $20^{\prime \prime}$ are mapped via the optical stellar and gaseous 
data, providing the missing link between the large-scale H I disk and the nuclear regions kinematic properties. Therefore, for the 7 pairs of Seyfert/control galaxies and the two single Seyfert galaxies NGC 1068 and NGC 3227 of the SAURON/Seyfert survey, for which H I data are also available via the VHIKINGS survey, full recovery of the kinematic parameters, from the outskirts of the galactic disk (via H I data) down to the inner regions (via optical data) is possible.

As reviewed above, numerous tools are available to derive the kinematic parameters via tilted-ring fits of the velocity fields. However, many of these tools, such as ROTCUR, are dedicated to radio interferometers data and required the installation of the entire softwares suite (e.g. GIPSY). In our study, we needed a small and handy tool to perform robust automated tilted-ring fits on either our radio or optical velocity fields and for which the characteristics of the fit are known. We developed then the PyRing tool using Python, taking into account the significant advantages of this language. Python is a dynamic object-oriented and portable programming language. It offers strong support for integration with other languages and tools and comes with extensive standard libraries. Moreover, Python codes are more maintainable (relative e.g. to C codes) and can be updated easily.

In the following, I present this tool and the first results we obtained by applying PyRing on our velocity fields.

\subsubsection{The PyRing tool}

We use the expression of the velocity (Eq. 5.4) with the tilted-ring method in order to derive the stellar and gaseous kinematic parameters. Our technique is inspired from ROTCUR (GIPSY). We assume the galactic disk to be geometrically thin, dominated by circular rotation, the deviation from axisymmetry being small. The galactic disk is divided into concentric ellipses determined by five parameters : the centre $\left(x_{c}, y_{c}\right)$, position angle $\Phi_{0}$, inclination $i$ and the offset velocity $V_{\text {sys }}$.

The velocity harmonic coefficients $c_{n}$ and $s_{n}$ are assumed to be constant within the rings width and they are then fitted on each ring, following Eq. 5.4. The tilted-ring method allows therefore to determine the harmonic coefficient of the velocity fields (related to the kinematic parameters) as a function of radius. Model velocity fields can then be constructed from the fitted values of the kinematic harmonic coefficients and then residual velocity fields can be derived.

The algorithm of PyRing is the same as the ROTCUR one. For each ring, we first determine the pixels belonging to the ring. The model velocities are computed for these pixels using the values of the input kinematic and geometrical parameters. Then these parameters are adjusted via a least-squares minimisation. A Levenberg-Marquardt least-squares minimisation routine implemented in python is used. It is based on the lmfit Fortran routine (part of 
the MINPACK-1 package). The $\chi^{2}$ is evaluated as

$$
\chi^{2}=\sum_{k} \frac{\left(O_{k}-M_{k}\right)^{2}}{w_{k}},
$$

where $k$ runs on all the pixels of the two-dimensional velocity map, $\mathrm{O}$ and $\mathrm{M}$ are the observed and model maps, respectively. $w$ is the weight map, generally assimilated with the rms noise of the original map: $w=\sigma_{r m s}^{2}$. In our approach, we assumed the noise in our velocity maps to be dominated by the photon noise. We assimilated then the weight map $w$ to the total intensity map. The expression of the $\chi^{2}$ computed in ROTCUR is slightly different since the weighting $w$ is not the same.

The $\chi^{2}$ minimisation computes formal errors. In order to take into account the correlation between the pixels of the velocity maps, these errors were multiplied by a factor

$$
\beta=\sqrt{\frac{4 \pi \sigma_{x} \sigma_{y}}{\delta_{x} \delta_{y}}},
$$

where $\delta_{x}, \delta_{y}$ are the size of a pixel and $\sigma_{x}, \sigma_{y}$ are the dispersion of the beam, in arcseconds (Schoenmakers et al., 1997). For the optical data, $\sigma_{x}, \sigma_{y}$ were assumed to be the dispersion of the PSF of the images $\left(\sigma_{P S F}=1.22 \times\right.$ seeing, where seeing is listed in Table 3.5).

In this work we chose a constant ring width at all radii, such that rings are large enough to have a sufficiently high number of pixels inside each ring so the fit is good, and small enough to assume that the harmonic coefficients are constant within a ring. Then the kinematic parameters are fitted interactively within each ring. First, the geometrical parameters (PA, $i$, dynamical centre), the systemic velocity $V_{\text {sys }}$ and the circular velocity $V_{c}$ are adjusted, under the simple assumption of pure circular motions, defined by Eq. 5.3. This fit allows to construct the velocity curve and it is done following several iterations:

1. Initial values for $V_{\text {sys }}, \Phi_{0}$ and $i$ were taken from previous published studies or the NASA Extragalactic Database (NED). We fixed the dynamical centre to coincide with the photometric centre, or with the location of the central radio continuum peak for the Seyfert galaxies.

2. A first fit is done, by fixing $\Phi_{0}$ and $i$ while allowing $V_{s y s},\left(x_{c}, y_{c}\right)$ and $V_{c}$ (the circular velocity) to vary as a function of $\mathrm{R}$. The mean values of $V_{\text {sys }}$, $x_{c}$ and $y_{c}$ are taken as improved estimates for the systemic velocity and the dynamical centre of the galaxy.

3. Then, we fit the position angle $\Phi_{0}$ and $V_{c}$ with $V_{s y s}$ and $\left(x_{c}, y_{c}\right)$ fixed at the values derived from the previous step, and the disk inclination fixed at its initial guess 
These three steps were followed for the stellar velocity fields and the H I velocity fields. For the SAURON velocity fields of the ionised gas, we imported the values of $V_{\text {sys }}$ and $\left(x_{c}, y_{c}\right)$ derived from the first step of the fit applied on the stellar components. Then we fitted the PA and the rotational velocity as for the stars. For the Seyfert galaxies, the regions dominated by the AGN were excluded as it has been done in Section 5.1.

\subsubsection{Testing PyRing}

In this section, I compare the results of our Python code PyRing with two well known velocity fit packages: GAL (in AIPS) and RESWRI (in GIPSY). In the case of the AIPS task GAL, a tilted-ring fit is not performed automatically. Therefore we interactively applied GAL in the tilted-ring way: we fit the velocity field with GAL in a set of rings, with the assumption of a constant rotation velocity within each ring.

Three tests were carried out. First I fitted a modeled velocity field, in pure circular rotation, with known parameters, with these three methods and compared the results of each fit with the initial parameters, and between each others. Then I applied GAL, RESWRI and PyRing on an H I observed velocity field and then on a SAURON velocity field and I compared the results of the three fits.

In the following I describe these three tests in details. A conclusion of these tests can be found in the Section 5.2.3.

\section{- Test on a model velocity field.}

In order to construct such a pure rotational velocity field, I run ROTCUR once on the observed H I velocity map of NGC 5364 (see Fig. D.1e) to derive the kinematic parameters as a function of radius. Then the VELFI routine (GIPSY) allows to re-construct a model velocity field with pure circular rotation from the initial kinematics parameters:

- Systemic velocity: $\mathrm{V}_{\text {sys }}=1234 \mathrm{~km} \mathrm{~s}^{-1}$

- Dynamical centre: RA $=13^{\mathrm{h}} 56^{\mathrm{m}} 12^{\mathrm{s}} \cdot 07, \mathrm{DEC}=5^{\mathrm{o}} 0^{\prime} 50.65^{\prime \prime}$

- Disk inclination: $i=43^{\circ}$

23 rings were used from 25 to $245^{\prime \prime}$ to construct the velocity field of a pure circular rotation with VELFI, using values of $\mathrm{V}_{c}$ and PA determined via ROTCUR. Fig. 5.7 shows the observed (left panel) and model (right panel) velocity fields of NGC 5364.

Then, we fit this circular velocity field with GAL, RESWRI and PyRing. The means and standard deviations over the radii of the best fitting values of the 

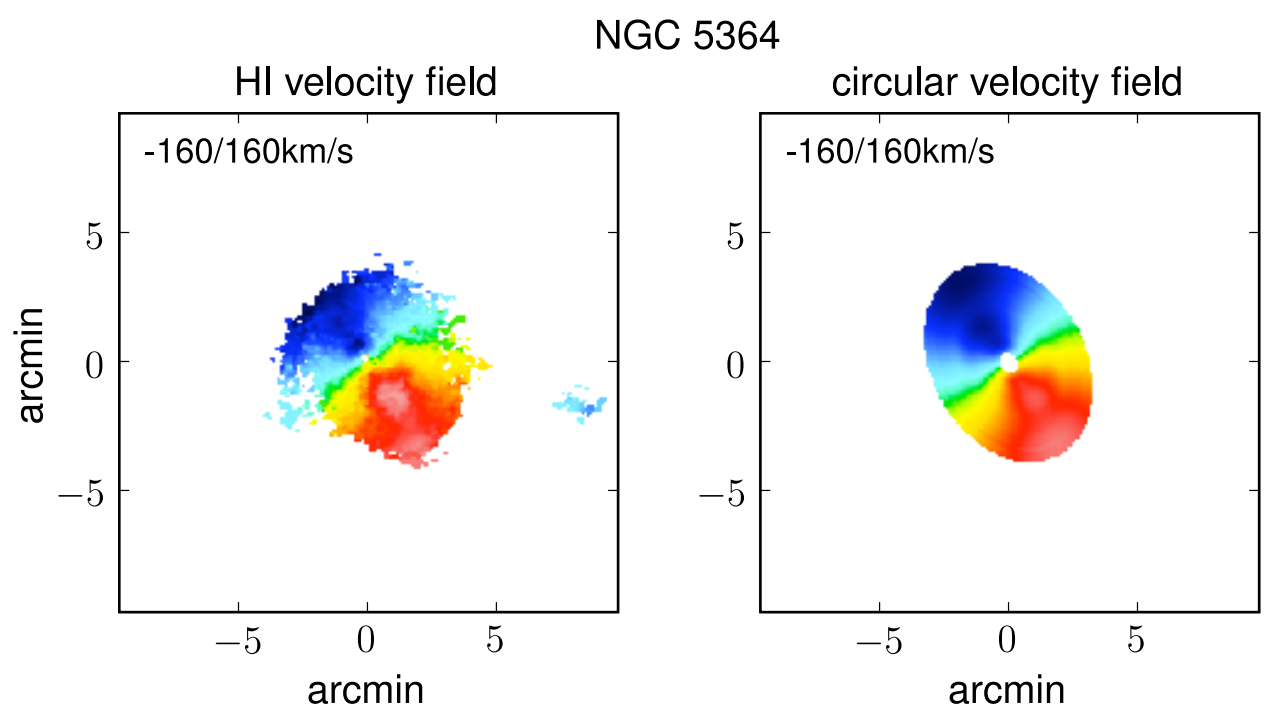

Figure 5.7: Observed H I velocity field (left panel) and circular velocity field (right panel) of NGC5364. The circular velocity field has been constructed with the GIPSY routine VELFI, using the best fitting kinematic parameters determined via ROTCUR.

\begin{tabular}{|c|c|c|c|}
\hline \multicolumn{5}{|c|}{ NGC 5364 model circular velocity field } \\
\hline Initial & GAL & RESWRI & PyRing \\
\hline \multicolumn{4}{|c|}{$\mathrm{V}_{\text {sys }}\left(\mathrm{km} \mathrm{s}^{-1}\right)$} \\
\hline 1234 & $1233.8 \pm 0.1$ & $1233.92 \pm 0.06$ & $1234.8 \pm 0.1$ \\
\hline \multicolumn{4}{|c|}{ dynamical centre } \\
\hline RA $13^{\mathrm{h}} 56^{\mathrm{m}} 12^{\mathrm{s}} .07$ & $13^{\mathrm{h}} 56^{\mathrm{m}} 12^{\mathrm{s}} .04 \pm 0^{\mathrm{s}} .01$ & $13^{\mathrm{h}} 56^{\mathrm{m}} 12^{\mathrm{s}} .089 \pm 0^{\mathrm{s}} .002$ & $13^{\mathrm{h}} 56^{\mathrm{m}} 12^{\mathrm{s}} .00 \pm 0^{\mathrm{s}} .02$ \\
DEC $5^{\circ} 0^{\prime} 50.7^{\prime \prime}$ & $5^{\mathrm{o}} 0^{\prime} 50.0^{\prime \prime} \pm 0^{\prime \prime} .4$ & $5^{\circ} 0^{\prime} 44.4^{\prime \prime} \pm 0^{\prime \prime} .07$ & $5^{\circ} 0^{\prime} 49.3^{\prime \prime} \pm 0^{\prime \prime} .4$ \\
\hline \multicolumn{4}{|c|}{$\mathrm{i}(\mathrm{deg})$} \\
\hline 43 & $42.8 \pm 0.5$ & $42.7 \pm 0.7$ & $42.9 \pm 0.1$ \\
\hline
\end{tabular}

Table 5.2: Estimation of the dynamical parameters of the H I modeled velocity field of NGC 5364, using three different fit routines : the GAL task of the AIPS package (second column), the RESWRI task of GIPSY (third column) and our PyRing code (fourth column). The first column lists the parameters of the input velocity field. . 

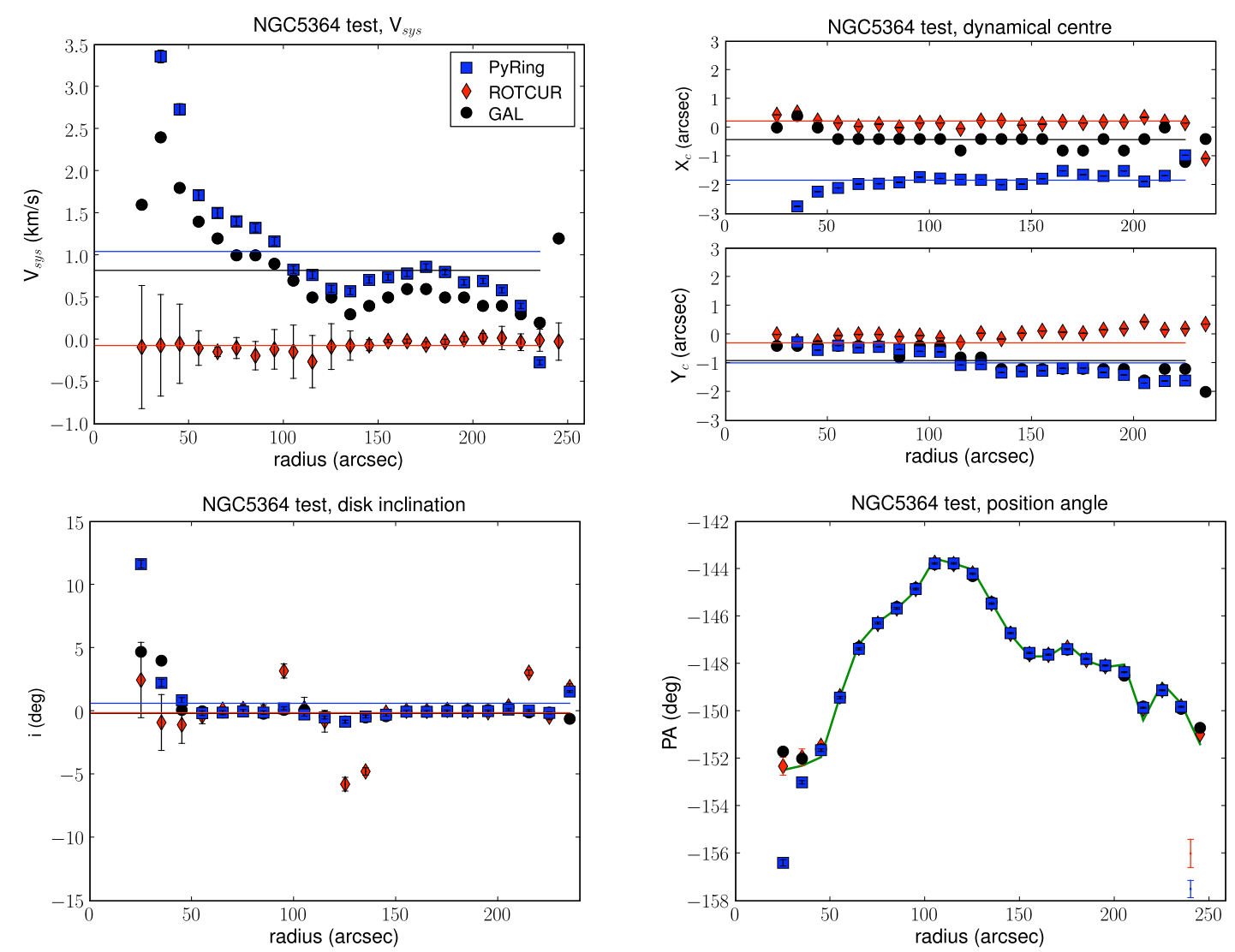

Figure 5.8: Comparison between AIPS, GIPSY and Python tilted-ring fits on the modeled velocity field of NGC 5364. The blue squares, black circles and red diamonds correspond to the Python, AIPS and GIPSY tilted ring fits, respectively for the systemic velocity (upper left figure), dynamical centre (upper right figure), disk inclination (lower left figure) and position angle (lower right figure). The systemic velocity, centre and inclination values are relative to the initial values taken to construct the modeled velocity field. The green line in the PA plot corresponds to the initial values of the PA. In each figure, the black, red and blue solid lines correspond to the mean value of the corresponding parameter determined by Python, GIPSY and AIPS fit, respectively. 

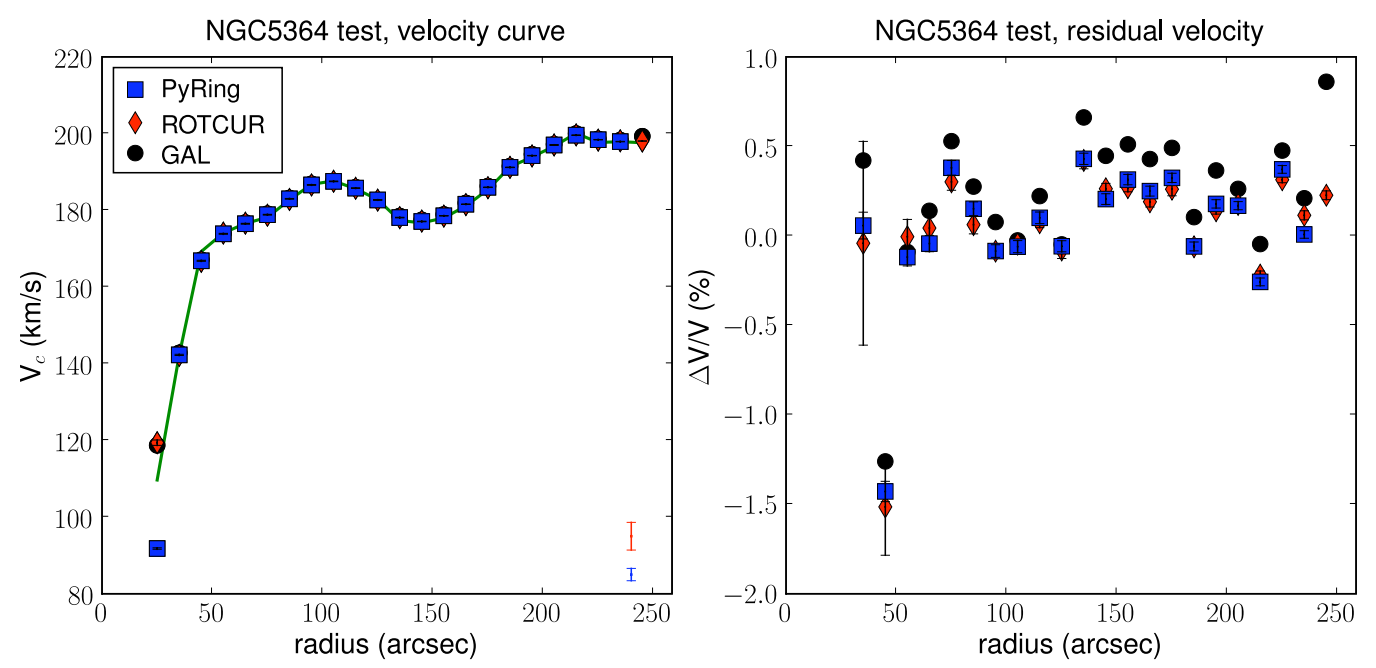

Figure 5.9: Comparison of AIPS, GIPSY and Python tilted ring fits on the modeled velocity field of NGC5364. In the left panel are shown the rotational velocities and in the right panel, the residuals expressed as : $\frac{V_{c}(\text { fit })-V_{c}(\text { initial })}{V_{c}(\text { initial })}$, where $V_{c}($ initial $)$ correspond to the initial values of $V_{c}$ and $V_{c}(f i t)$ to the fitted ones. The blue squares,blue circles and red diamonds correspond to the Python, AIPS and GIPSY estimation of the rotation velocity, respectively. The green line corresponds to the initial values of $V_{c}$. In the left panel, error bars are too small to be seen, compared to the symbol size. Typical values of those error bars are shown in the bottom right corner, for RESWRI (red line) and PyRing (blue line). These vertical lines correspond to 50 times the mean values of the error bars computed in each fit. In the case of GAL (AIPS) no error bars are available. 
systemic velocity, dynamical centre, disk inclination and PA for the three methods are listed in Tab. 5.2. This table shows the initial values of these parameters for comparison (first column). Fig. 5.8 shows the radial variations of the systemic velocity $\mathrm{V}_{s y s}$, dynamical centre $\left(\mathrm{x}_{c}, \mathrm{y}_{c}\right)$, disk inclination $i$ and kinematic position angle PA, derived from the three methods. The average values over the radii of $\mathrm{V}_{s y s},\left(\mathrm{x}_{c}, \mathrm{y}_{c}\right)$ and $i$ are shown as horizontal lines in the first three figures. All the values are relative to the initial values of these dynamical parameters. In the plot of PA, the initial PA values are plotted as a green line.

The larger deviations between the fitted and initial values occur at small radii $\left(\mathrm{R}<50^{\prime \prime}\right.$, especially for the PyRing fit), essentially for $V_{\text {sys }}$ (top left plot), and only for GAL and PyRing. This may be a bias from the test, since the circular velocity field was constructed using parameters derived with ROTCUR. In the case of $V_{s y s}$, the variations of the PyRing and GAL fitted values are very similar, the PyRing values being always larger than GAL values by about 0.5 $\mathrm{km} \mathrm{s}^{-1}$. The amplitude of these variations is about $3.5 \mathrm{~km} \mathrm{~s}^{-1}$ for both GAL and PyRing and the average value of the systemic velocity fitted by these too method is about $1 \mathrm{~km} \mathrm{~s}^{-1}$ too high then the initial value.

Considering the determination of the dynamical centre (Fig. 5.8, top right plot), the radial variations remain very small (less than $2.5^{\prime \prime}$ for all the fitting tools) and the mean values of $x_{c}$ and $y_{c}$ are equal to the initial values within $1^{\prime \prime}$, for all fitting methods. Similarly, despite some accidents at few radii, the fitted values of $i$ and PA (Fig. 5.8, left and right bottom plot, respectively) are very close to the initial values, especially for PA, for which the points obtained from the three tools almost overlap perfectly. These radial variations and the deviations from the initial values are very small compared to the spatial resolution of our optical and radio data.

Finally, Fig. 5.9 presents the velocity curve of NGC 5364 determined using the three methods, with the geometric parameters and $V_{\text {sys }}$ kept at their mean values (Tab. 5.2). We notice that except for the two first radii, the estimations of the three methods are in very good agreement with the initial rotation curve, and with each others.

\section{- Test on an observed $\mathrm{H}$ I velocity field.}

The test on a circular rotation velocity field, shows that the three fitting methods GAL, RESWRI and PyRing can robustly adjust the geometric and kinematic parameters, and that there is no systematic error between the three fits. This test was repeated on the observed H I velocity field of NGC5364, which could contain non-circular motions. This second test allows to compare the behaviour of the three methods when non-circular motions are present. Fig. 5.10 shows the radial variations of the systemic velocity, dynamical centre, disk inclination and PA values estimated by GAL, RESWRI and PyRing, and Fig. 5.11 presents the rotation curve of NGC5364 derived using either RESWRI, GIPSY or PyRing, with the mean values of $V_{s y s}, x_{c}, y_{c}$ and $i$ listed in Table 5.3. 

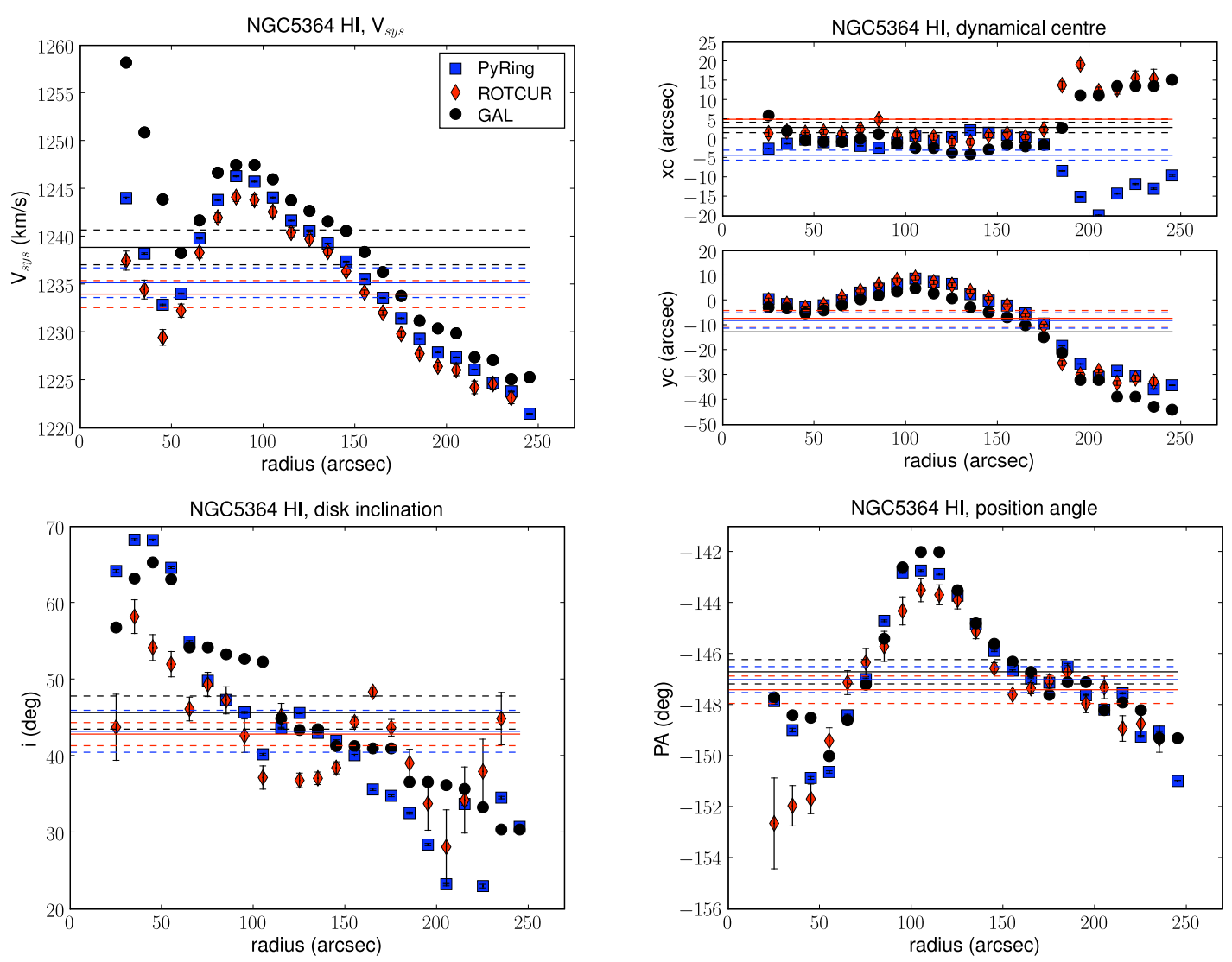

Figure 5.10: Comparison of GAL (black circles), RESWRI (red diamonds) and PyRing (blue squares) tilted-ring fits on the observed H I velocity field of NGC 5364 for the systemic velocity (upper left figure), dynamical centre (upper right figure), disk inclination (lower left figure) and position angle (lower right figure). In each figure, the black, red and blue solid lines correspond to the mean value of the corresponding parameter determined by Python, GIPSY and AIPS fit, respectively. The two corresponding dashed lines represent the associated upper and lower limits at $1 \sigma$. For the dynamical centre (top right panel), the origin $\left(\mathrm{x}_{c}, \mathrm{y}_{c}\right)=(0,0)$ corresponds to the coordinates of the centre of NGC $5364\left(13^{\mathrm{h}} 56^{\mathrm{m}} 12^{\mathrm{s}} .0,+05^{\circ} 00^{\prime} 52^{\prime \prime}\right.$, from NED). . 


\begin{tabular}{|c|c|c|}
\hline \multicolumn{3}{|c|}{ NGC 5364 H I velocity field } \\
\hline \multicolumn{3}{|c|}{$\mathrm{V}_{\text {sys }}\left(\mathrm{km} \mathrm{s}^{-1}\right)$} \\
\hline GAL & $1234.0 \pm 1.4$ & $1235.3 \pm 1.2$ \\
\hline $1238.9 \pm 1.8$ & dynamical centre \\
\hline \multicolumn{3}{|c|}{ PyRing } \\
\hline $\begin{array}{c}\text { RA } 13^{\mathrm{h}} 56^{\mathrm{m}} 12^{\mathrm{s}} 19 \pm 0^{\mathrm{s}} .07 \\
\text { DEC } 5^{\mathrm{o}} 0^{\prime} 44^{\prime \prime} \pm 3^{\prime \prime}\end{array}$ & $13^{\mathrm{h}} 56^{\mathrm{m}} 12^{\mathrm{s}} 3 \pm 0^{\mathrm{s}} .1$ & $13^{\mathrm{h}} 56^{\mathrm{m}} 11^{\mathrm{s}} .7 \pm 0^{\mathrm{s}} .1$ \\
$5^{\mathrm{o}} 0^{\prime} 45^{\prime \prime} \pm 3^{\prime \prime}$ & $5^{\mathrm{o}} 0^{\prime} 45^{\prime \prime} \pm 3^{\prime \prime}$ \\
\hline \multicolumn{3}{|c|}{$\mathrm{i}(\mathrm{deg})$} \\
\hline $45.7 \pm 2.2$ & $42.9 \pm 1.9$ & $43.2 \pm 2.7$ \\
\hline \multicolumn{3}{|c|}{ PA (deg) } \\
\hline$-147.4 \pm 0.5$ & $-146.7 \pm 0.5$ & $-147.0 \pm 0.5$ \\
\hline
\end{tabular}

Table 5.3: Estimation of the dynamical parameters of the observed H I velocity field of NGC 5364 using three different fitting routines : GAL task of the AIPS package (first column), RESWRI task of GIPSY (second column) and PyRing (third column).

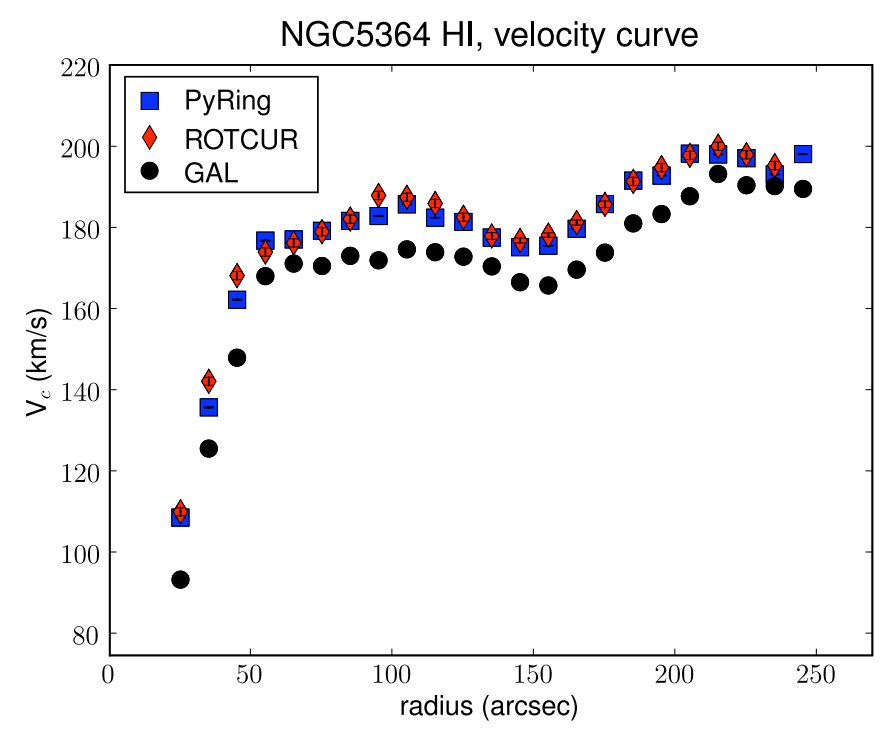

Figure 5.11: Comparison of the velocity curves of NGC 5364 estimated via GAL (black circles), RESWRI (red diamonds) and PyRing (blue squares) 


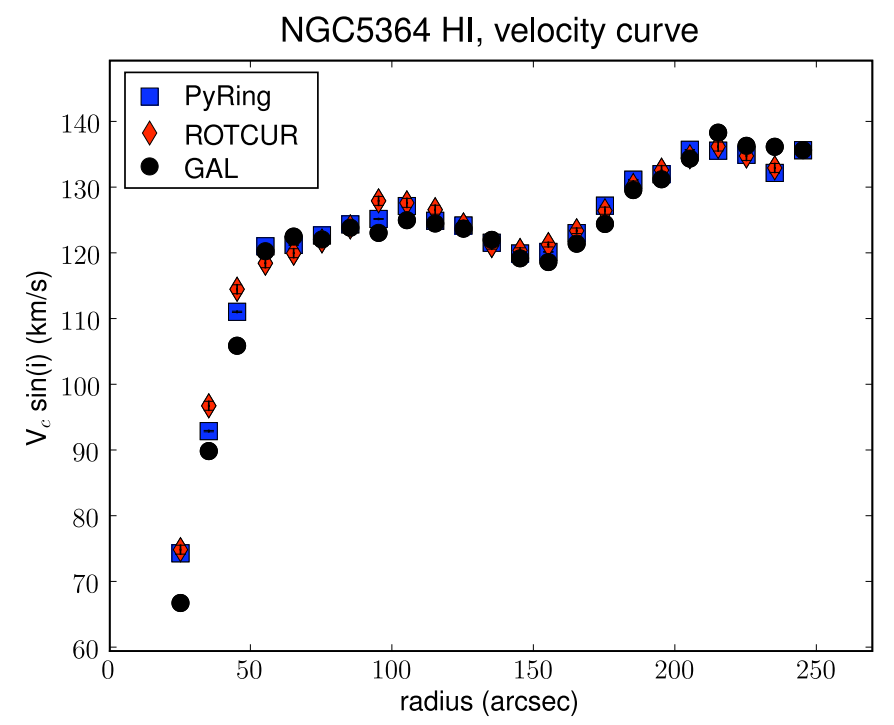

Figure 5.12: Comparison of the projected velocity curves of NGC5364 estimated via GAL (black circles), RESWRI (red diamonds) and PyRing (blue squares). Disk inclinations are $45.7^{\circ}, 42.9^{\circ}$ and $43.2^{\circ}$ for GAL, RESWRI and PyRing, respectively (see Tab. 5.3)

We can notice that the three methods are in good agreement, the mean values of the geometric and kinematic parameters are equal, within their error bars, except for $x_{c}$ (Tab. 5.3). There is a significant but small difference between the values of $x_{c}$ found using PyRing and those determined with GAL and RESWRI. Indeed for radii larger than 180", GAL and RESWRI find values of $x_{c}$ about $15^{\prime \prime}$ above the estimated values for $\mathrm{R}<180^{\prime \prime}$, while PyRing fits values of $x_{c} 15^{\prime \prime}$ below the previous values. For $\mathrm{R}<180^{\prime \prime}$, GAL, RESWRI and PyRing are in very good agreement, finding similar values within $\sim 7^{\prime \prime}$, well below the beam size $\left(20^{\prime \prime} 5 \times 16^{\prime \prime} \cdot 7\right)$. Except for the case of $x_{c}$, the radial variations of the fitted values of $V_{\text {sys }},\left(x_{c}, y_{c}\right), i$ and PA derived with the three methods are very similar. The larger deviations between the three fitting routines occur for the estimations of the disk inclinations. The three fitted rotation curves $V_{c}$ derived by GAL, RESWRI or PyRing (Fig. 5.11) have the same shape, with a scatter less than $10 \mathrm{~km} \mathrm{~s}^{-1}$ between the different methods. GAL finds systematically lower values of $V_{c}$. This effect is due to the larger value of the disk inclination for GAL relatively to RESWRI or PyRing. When we plot the projected velocity curve $V_{c} \sin (i)$, the three curves estimated by PyRing, RESWRI and GAL merge (Fig. 5.12) 
- Test on an observed SAURON velocity field.

Finally, a last test was carried out on an SAURON velocity field. For this test, we applied PyRing and RESWRI on the stellar and gaseous velocity fields of NGC5055 (Fig. C.1b).

The systemic velocity, dynamical centre and disk inclination are first estimated from the stellar velocity field. Fig. 5.13 presents the radial variations of the $\mathrm{V}_{\text {sys }}$ (top left panel), $\mathrm{X}_{c}, \mathrm{Y}_{c}$ (top right panel) and $i$. In the bottom right panel of this figure is also shown the position angle of the stellar velocity field and Fig.5.14 shows the fitted stellar rotation curves (left panel) as well as the residuals between the PyRing and RESWRI fits (right panel). The RESWRI fit stops at smaller radii than the PyRing, since RESWRI cannot execute the fit on the rings which go out of the velocity field, even if only a small part of the ring is outside the field-of-view. The velocity field of NGC 5055 is divided into concentric rings as shown in Fig. 5.5. As one can see in this figure, some parts of the last three rings are outside of the velocity field, then RESWRI fit the kinematic parameters everywhere but in these rings, while PyRing can be applied in all the field-of-view. In the range of radii common to the RESWRI and PyRing fits, the RESWRI and PyRing estimations of the kinematic and geometrical parameters are in good agreement.

Table 5.4 lists the average values estimated by RESWRI (first column) and PyRing (second column) of the systemic velocity, dynamical centre, inclination and stellar kinematic position angle. The differences between the two methods are of the same order as those observed in the fits of H I velocity fields (see Table 5.3). The rotation curves fitted by PyRing and RESWRI are shown in the left panel of fig. 5.14. The two methods are in very good agreement, the differences (right panel of fig. 5.14) being less than $5 \mathrm{~km} \mathrm{~s}^{-1}$.

Finally PyRing and RESWRI were applied on the velocity field of the ionised gas of NGC 5055, using the value of the kinematic parameters estimated from fits on the stellar velocity field. Fig. 5.15 presents the results of these two fits: the gaseous kinematic PAs in the left panel and the gaseous rotation curves derived by PyRing (blue squares) and RESWRI (red diamonds). Like for the stellar component, the estimations of the PA and the rotation velocity via the two fits are in very good agreement. 

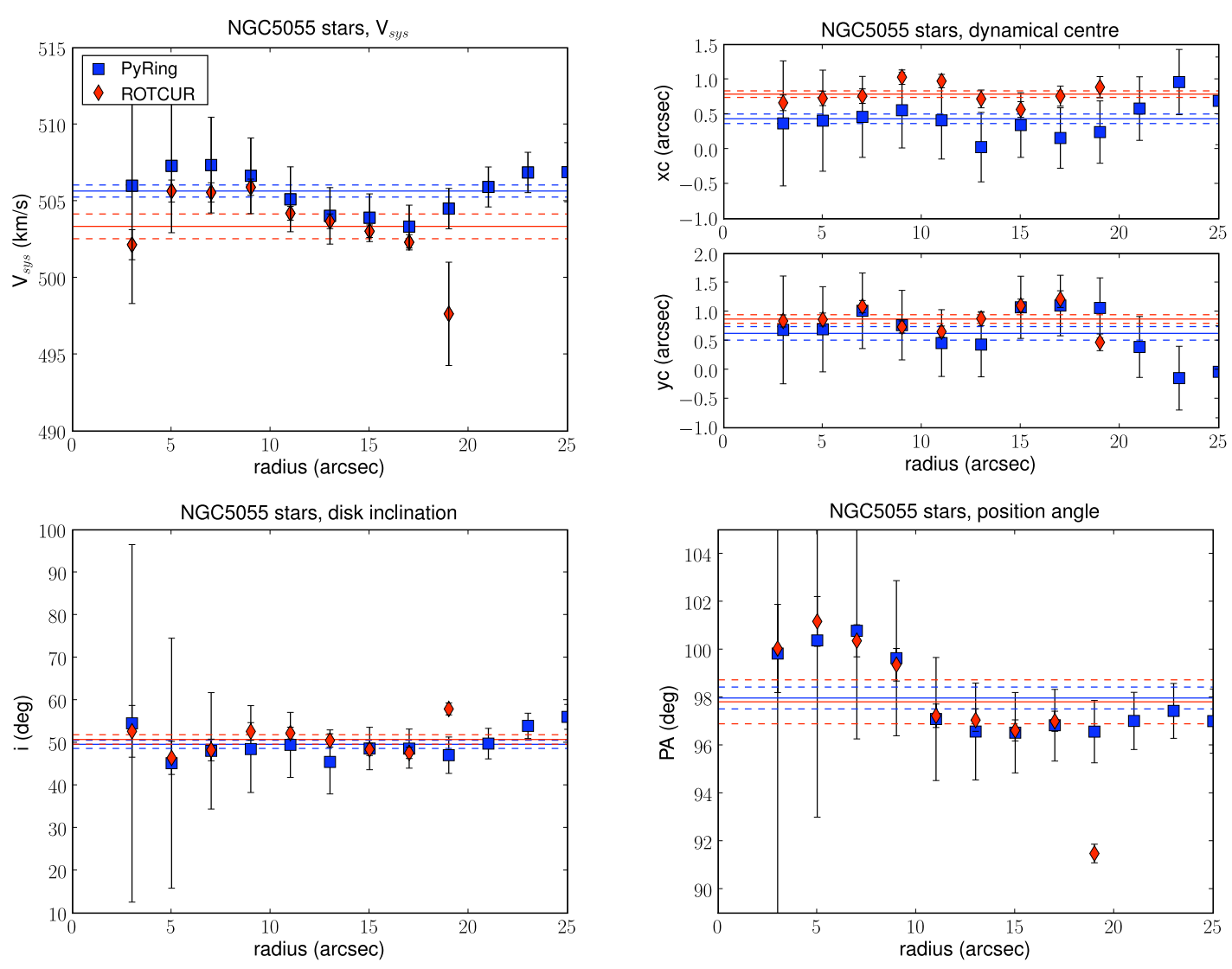

Figure 5.13: Comparison between GIPSY and Python tilted-ring fits on the stellar velocity field of NGC 5055. The blue squares and red circles diamonds correspond to the Python and GIPSY tilted ring fits, respectively for the systemic velocity (upper left figure), dynamical centre (upper right figure), disk inclination (lower left figure) and position angle (lower right figure). The systemic velocity, centre and inclination values are relative to the initial values taken to construct the modeled velocity field. The green line in the PA plot corresponds to the initial values of the PA. In each figure, the red and blue solid lines correspond to the mean value of the corresponding parameter determined by PyRing and RESWRI respectively. The two corresponding dashed lines represent the associated upper and lower limits at $1 \sigma$. . 


\begin{tabular}{|c|c|}
\hline \multicolumn{2}{|c|}{ NGC 5055 stellar velocity field } \\
\hline RESWRI & PyRing \\
\hline \multicolumn{2}{|c|}{$\mathrm{V}_{\text {sys }}\left(\mathrm{km} \mathrm{s}^{-1}\right)$} \\
\hline $503.4 \pm 0.8$ & $505.7 \pm 0.4$ \\
\hline dynamical centre \\
\hline RA 13 $15^{\mathrm{h}} 49^{\mathrm{s}} 25 \pm 0^{\mathrm{s}} 003$ & $13^{\mathrm{h}} 15^{\mathrm{m}} 49^{\mathrm{s}} 27 \pm 0^{\mathrm{s}} .004$ \\
DEC $42^{\circ} 01^{\prime} 45.87^{\prime \prime} \pm 0^{\prime \prime} .07$ & $42^{\circ} 01^{\prime} 45.6^{\prime \prime} \pm 0^{\prime \prime} .1$ \\
\hline \multicolumn{3}{|c|}{$\mathrm{i}(\mathrm{deg})$} \\
\hline $50.8 \pm 1.1$ & $49.6 \pm 1.0$ \\
\hline \multicolumn{3}{|c|}{ PA (deg) } \\
\hline $97.8 \pm 0.9$ & $98.0 \pm 0.5$ \\
\hline
\end{tabular}

Table 5.4: Estimation of the dynamical parameters of the observed stellar velocity field of NGC 5364 using tow fitting routines : RESWRI task of GIPSY (first column) and PyRing (second column).
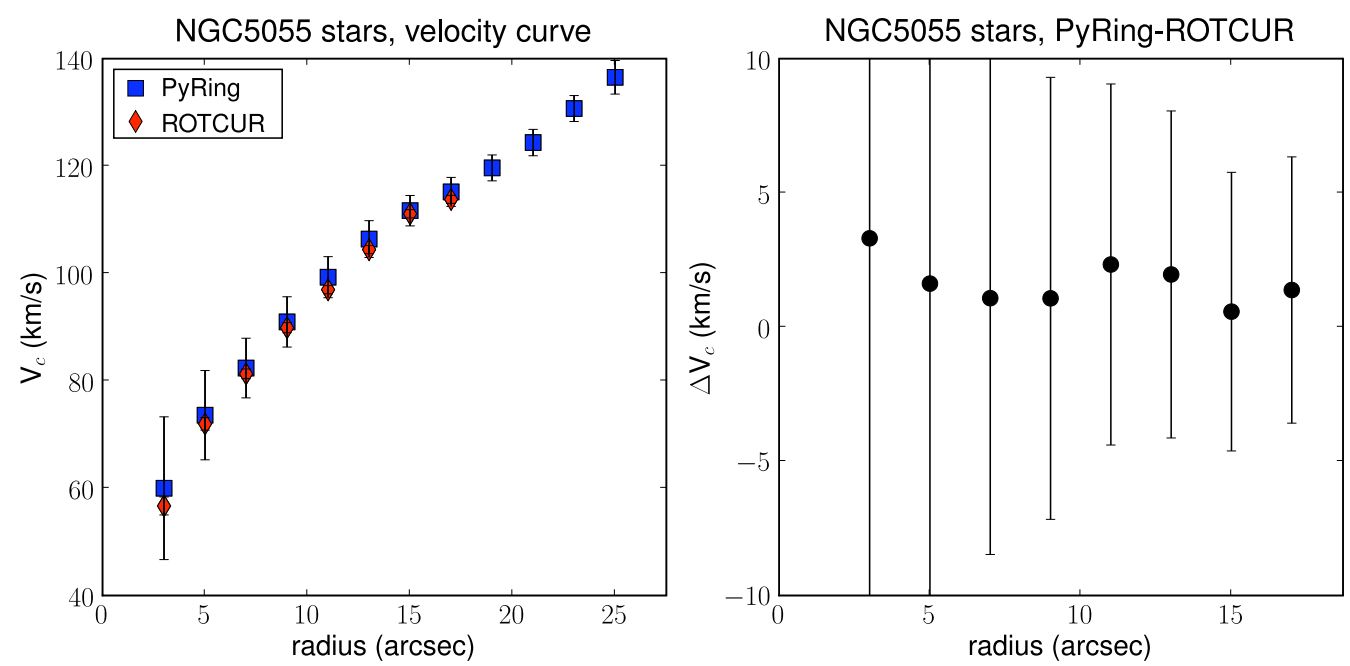

Figure 5.14: Comparison of the velocity curves of the stellar component of NGC 5055 estimated with RESWRI (red diamonds) and PyRing (blue squares). The rotation velocities are shown in left panel. The right panel presents the differences between the circular velocities estimated by PyRing and RESWRI. 

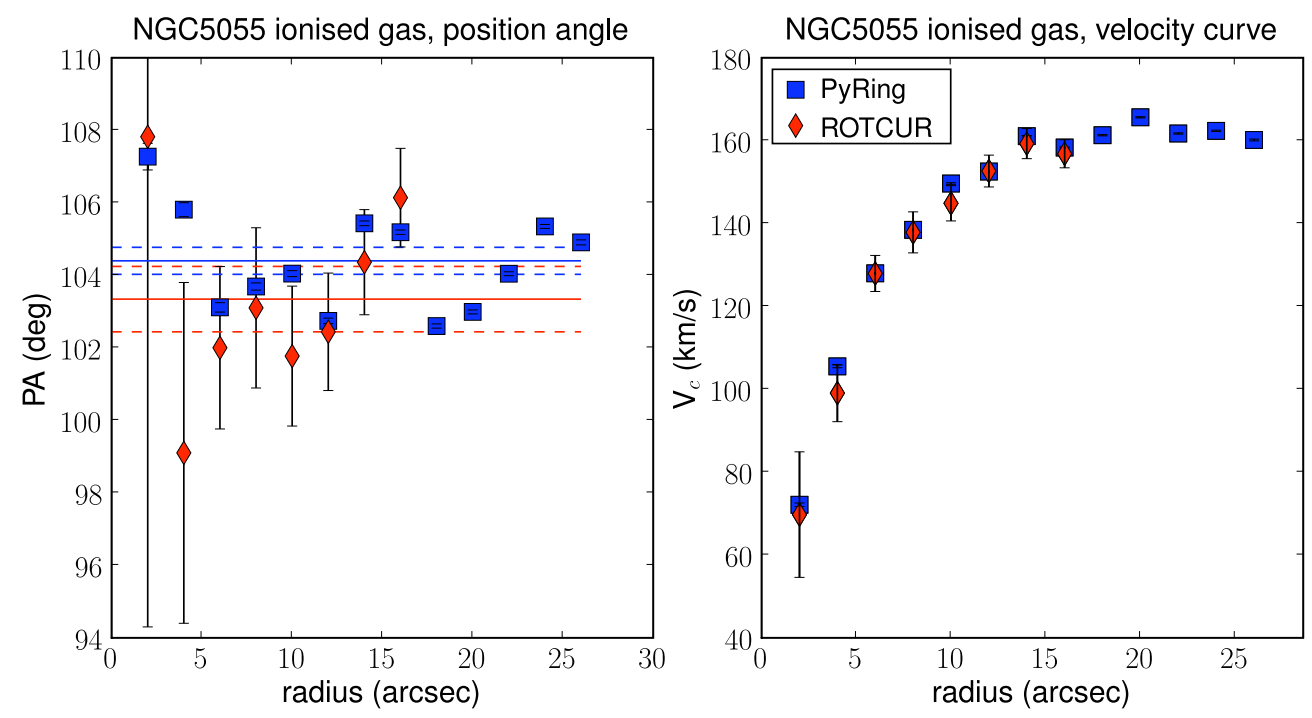

Figure 5.15: Comparison of kinematic PA and the velocity curves of the gaseous component of NGC 5055 estimated with RESWRI (red diamonds) and PyRing (blue squares). The kinematic PAs are shown in the left panel. The red and blue solid lines correspond to the mean PA determined by PyRing and RESWRI respectively. The two corresponding dashed lines represent the associated upper and lower limits at $1 \sigma$. Rotation velocities are shown in the right panel. 


\subsubsection{Conclusion from these tests.}

These two tests, performed to compare our PyRing tilted-ring fit with the wellused methods GAL (AIPS) and RESWRI (GIPSY), show that:

- When fitting a pure rotation velocity field, PyRing finds best-fitting geometrical and kinematic parameters similar to the initial values and to the fitted values determined using GAL and RESWRI (Figs. 5.8, 5.9 and Table 5.2).

- The larger differences between the fitted PyRing values and the initial values on the kinematic parameter for the pure rotation velocity field occur in the very central regions where the fit may be the less robust due to too few pixels within a ring (Figs. 5.8 and 5.9).

- In the case of an observed H I or optical velocity field fit, PyRing is in very good agreement with RESWRI and GAL (in the case of the H I velocity fields) (Figs. 5.10, 5.11, 5.13 and 5.14 and Tables 5.3 and 5.4).

- For the fit of the H I velocity field, the largest differences between the PyRing, GAL and RESWRI values occur in the innermost regions, in particular for $V_{\text {sys }}$, PA and $V_{c}$ (Figs. 5.10 and 5.11).

- The error bars of the fits of the optical data are greater than those of the fit of the H I velocity fields, for both PyRing and RESWRI.

Therefore, these three tests show that our kinematic tool PyRing can robustly fit the parameters of the two-dimensional $\mathrm{H}$ I and optical velocity fields.

\subsection{Radial Variations of Kinematic Properties}

In this section we present the results of the PyRing fit performed on our SAURON (Sec. 5.3.1) and H I (Sec. 5.3.2) velocity fields, assuming first a pure circular rotation. The global rotation curve for the galaxies with both radio and optical data available are presented in Sec. 5.3.3

\subsubsection{PA and rotation curve in the central regions}

The results of this first analysis on the SAURON velocity fields are presented in this section. For each galaxy, the stellar and gaseous kinematic PAs (Figs. 5.16a and 5.16b) and rotation curves (Figs. 5.18a, 5.18b) are plotted as a function of radius. The values of the global stellar and gaseous kinematic PAs derived in Sec. 5.1 are indicated on each plot of Figs. 5.16a and 5.16b, as a dotted red and dashed blue lines, respectively. All angles are given relative to the photometric major-axis PA. 


\begin{tabular}{|l|l|l|}
\hline Pairs & $\begin{array}{l}\text { Name } \\
\text { NGC } \\
(1)\end{array}$ & $\begin{array}{c}\mathrm{R}_{e} \\
\mathrm{kpc} \\
(2)\end{array}$ \\
\hline \multirow{4}{*}{1} & 1068 & 3.1 \\
\cline { 2 - 3 } & 3227 & 4.3 \\
\hline \multirow{2}{*}{2} & 2655 & 3.7 \\
\cline { 2 - 3 } & 4459 & 3.1 \\
\hline \multirow{3}{*}{3} & 3627 & 2.3 \\
\cline { 2 - 3 } & 5806 & 2.9 \\
\hline \multirow{4}{*}{4} & 4051 & 5.0 \\
\cline { 2 - 3 } & 5249 & 5.9 \\
\hline \multirow{3}{*}{5} & 4151 & 2.9 \\
\cline { 2 - 3 } & 2985 & 3.1 \\
\hline \multirow{2}{*}{6} & 3579 & 3.9 \\
\hline & 3351 & 2.1 \\
\hline 7 & 5055 & 2.8 \\
\hline \multirow{2}{*}{7} & 6951 & 6.3 \\
\cline { 2 - 3 } & 5248 & 5.9 \\
\hline
\end{tabular}

Table 5.5: Effective radius $\left(\mathrm{R}_{e}\right)$ of the SAURON/Seyfert sample. The values of $\mathrm{R}_{e}$ are measured in the J-band 2MASS images (taken from Jarrett et al., 2003), except for NGC 4459 for which we used the value measured by Cappellari et al. (2006).

\section{- Radial variations of the kinematic PA}

The radial variations of the stellar and gaseous kinematic PA, shown in the top plots of Figs 5.16a and 5.16b reveal the change of orientation of the stellar and gaseous kinematic major-axis orientation as a function of radius, a potential hint for the presence of non-circular components. The global values of the kinematic PAs derived in Sec 5.1 only correspond to a small part of the FOV over which the kinematic orientation is globally constant. For NGC 3627, the global stellar and gaseous kinematic PAs derived in the previous Section correspond to values outside $r \gtrsim 10^{\prime \prime} \approx 300$ pc (Fig. 5.16a), and for NGC 5806, our first method is not sensitive to the variations of PAs between 10 and 15" (Fig. 5.16a). In the case of NGC 2655, the kinematic PA of the ionised gas rises abruptly for $\mathrm{r}<10^{\prime \prime}=1.2 \mathrm{kpc}$ from the value found by our global method to a value which tends to the stellar kinematic PA.

In order to quantify the variations in the radial profiles of the kinematic PAs, we have used the difference between the maximum and minimum values of the kinematic PAs. For each galaxy, the quantity $\triangle \mathrm{PA}=\mathrm{PA}_{\max }-\mathrm{PA}_{\min }$ is computed for the gaseous and stellar components. To compare the Seyfert hosts to their associated inactive galaxies, the maximum and minimum values of the kinematic PAs were derived on regions of $\mathrm{r}<\mathrm{R}_{e} / 3$, where $\mathrm{R}_{e}$ is the effective (half-light) radius. This radius is usually determined from NIR images 

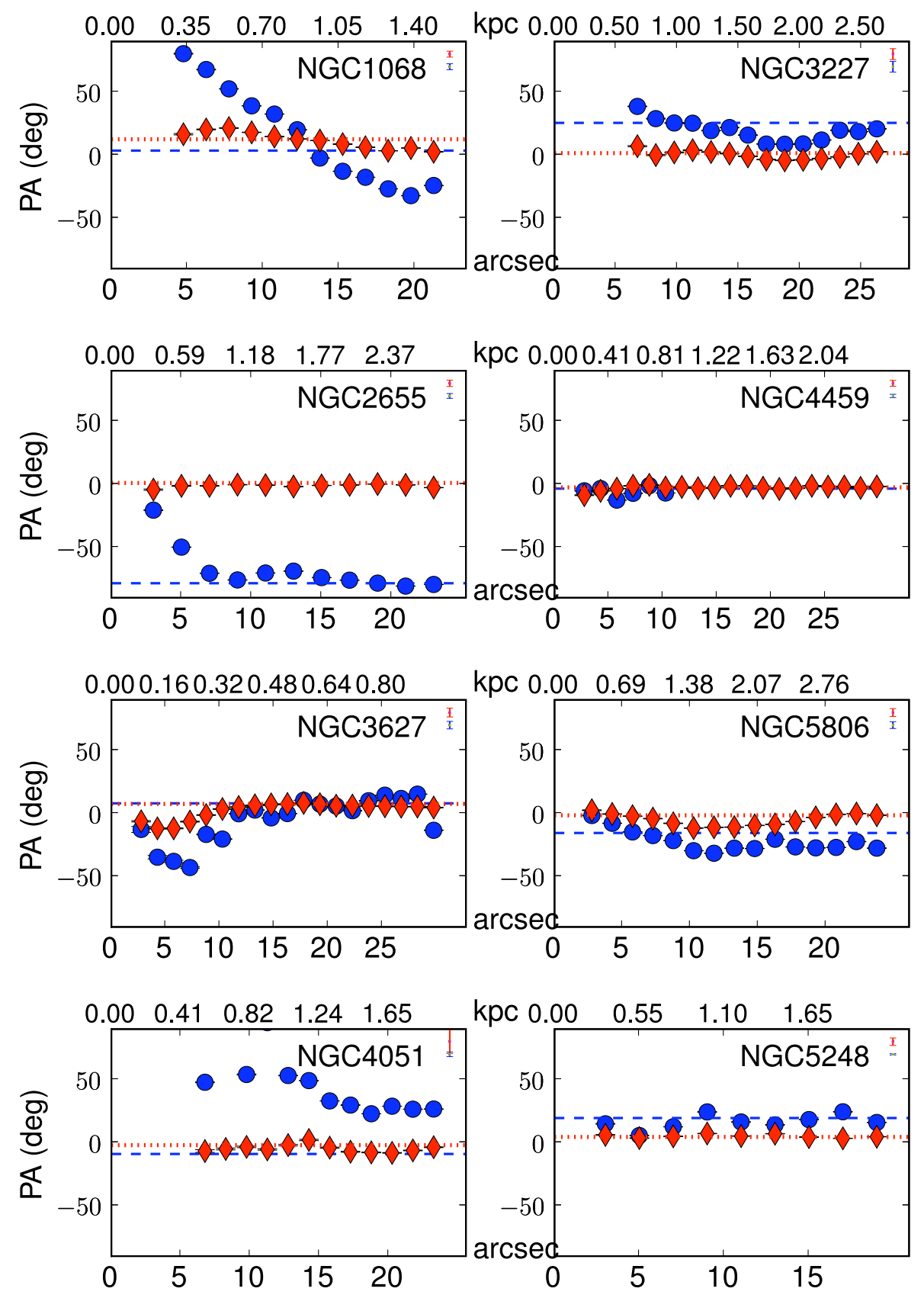

Figure 5.16a: Kinematic position angles as a function of radius of the stars (red diamonds) and of the gas (blue circles) components. The first row shows the Seyfert galaxies NGC 1068, and NGC 3227. Each subsequent row presents a pair of galaxies: the Seyfert on the left, and the inactive galaxy on the right. The regions related to the BLR of the Seyfert 1 galaxies (NGC 3227, NGC 4051 and NGC 4151) and to the NLR of NGC 1068 have been excluded from the fit. The dotted red and dashed blue horizontal lines represent the values of the global position angle of the kinematic major-axis for the stars and gas respectively. These values were derived from the velocity field symmetrization method (see Section 5.1). The values of the kinematic PA are relative to the photometric PA (see Table 5.1). The errorbars of each point are smaller than the size of the symbol, and then are not visible. The red and blue error bars drawn in the top right corner of each plot represent 20 times the typical errorbar of the stellar and gaseous PAs, respectively. 

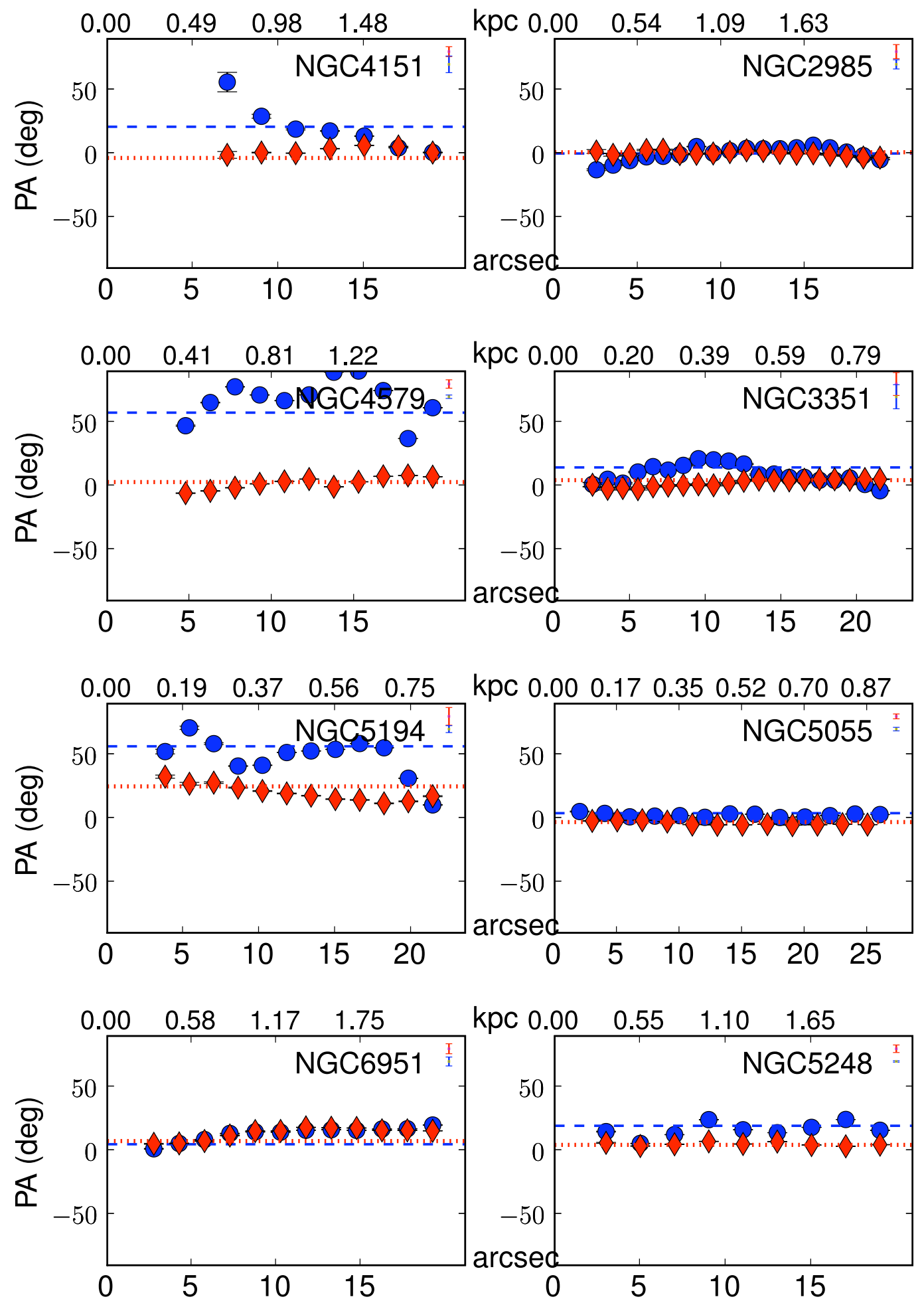

Figure 5.16b: Fig. 5.16a continued. 

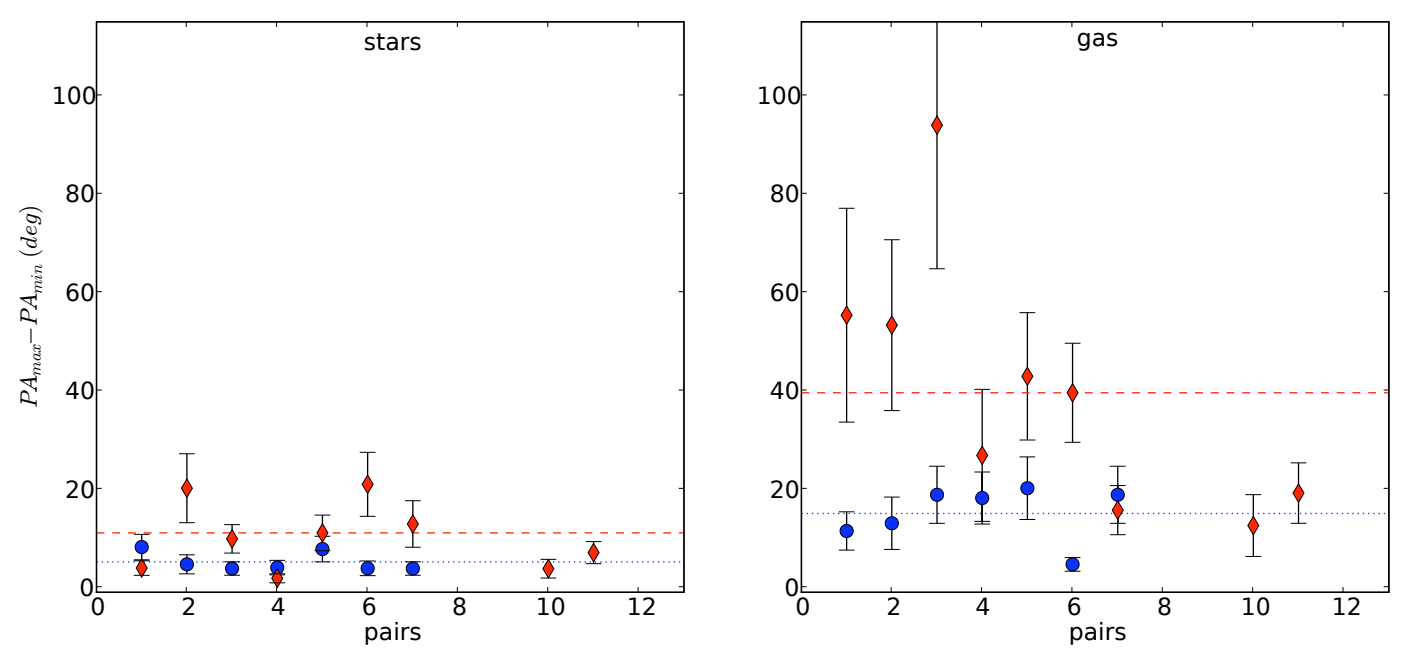

Figure 5.17: Distribution of the amplitude of variations of the stellar (left panel) and the gaseous (right panel) kinematic PAs for $\mathrm{R}<\mathrm{R}_{e} / 3$ (see text for details). Active galaxies and inactive galaxies are shown as filled red diamonds and filled blue circles respectively. The dotted blue and dashed red lines correspond to the average of $\mathrm{PA}_{\max }-\mathrm{PA}_{\min }$ for the inactive and Seyfert galaxies respectively, for the stellar (left panel) or gaseous (right panel) components. NGC 1068 and NGC 3227 have been excluded to compute the average values corresponding to the Seyfert galaxies, since they do not have an associated inactive galaxy.

which trace the older stellar population and which are less contaminated by star formation regions. Jarrett et al. (2003) determine the $R_{e}$ of galaxies from the 2MASS survey from a fit of a de Vaucouleurs luminosity function to the NIR radial luminosity. Twelve of our galaxies $(80 \%$ of the SAURON/Seyfert sample) are include in their sample. We adopt then $\mathrm{R}_{e}$ determined from the J-band 2MASS images (Jarrett et al., 2003) for these twelve galaxies.

NGC 4459, 5806 and 6951 are not in their sample. For NGC 4459 we used the value of $\mathrm{R}_{e}$ determined by Cappellari et al. (2006) and for NGC 5806 and 6951 we computed the half-light radius as the radius for which the growth curve of the J-band luminosity is divided by two. A fit of a de Vaucouleurs growth curve to the J-band 2MASS image has been also done. This fit gives a similar value of $\mathrm{R}_{e}$ in the case of NGC 5806. This is not the case of NGC 6951 because of the spiral arms which are still predominant in the NIR image of this galaxy. For these two galaxies, we then used the effective radius $\mathrm{R}_{e}$ determined directly from the growth curve. Table 5.5 lists the values of $R_{e}$ used in our study.

For each velocity field, $\triangle \mathrm{PA}$ is derived inside $\mathrm{R}_{e} / 3$ if the FOV extends beyond this radius. Otherwise, we measured $\triangle \mathrm{PA}$ inside the whole extent of the velocity field. In the case of the Seyfert galaxies, the regions which could present residual AGN-induced perturbations are excluded using the [O III] / $\mathrm{H} \beta$ line ratios, as it has been done for the determination of the global 
kinematic PA in the previous section (Sec. 5.1). The resulting $\triangle \mathrm{PA}$ values for both the gaseous and stellar components, and for all galaxies in our sample are provided in Fig. 5.17. Also shown are the average $\Delta$ PA of each component for the inactive galaxies (blue lines) and the Seyfert galaxies (red lines). For the latter, the averages are made excluding NGC 1068 and NGC 3227 since these two Seyferts do not have associated control galaxies.

The first result of this analysis comes from a comparison between the gaseous and stellar components: for both the Seyfert and inactive sub-samples, $\triangle \mathrm{PA}$ is almost always higher for the gas than for the stars. While the stellar kinematic PAs radial variations are restrained within about $20^{\circ}$ and $15^{\circ}$ for respectively the Seyfert and inactive galaxies, such variations of the gaseous PAs reach more than $50^{\circ}$ in the case of three Seyfert galaxies (NGC 2655, NGC 3627, NGC 4051). The average $\triangle \mathrm{PA}$ of the gaseous component is about 4 times greater than the average $\triangle \mathrm{PA}$ of the stars for the Seyfert galaxies as well as for the inactive galaxies.

The second result is obtained when comparing the Seyfert and inactive galaxies: for both gas and stars, $\triangle \mathrm{PA}$ is almost always larger for the Seyferts than for their control inactive galaxies. The average $\triangle \mathrm{PA}$ for the Seyferts is about 2.5 times greater than the average $\triangle \mathrm{PA}$ for the inactive galaxies, considering either the stellar or the ionised gas components.

\section{- The stellar and gaseous rotation curves.}

The top plots of Figs. 5.18a and 5.18b show the rotation curve of the stars and ionised gas in the central regions of our Seyfert and non-active galaxies. For all the galaxies, the stellar velocity curves are very regular and the maximum velocity seem not to be reached in the inner $\sim 20^{\prime \prime}$ except for NGC 4151 . We observe several shapes for the stellar velocity curves:

1. For three Seyferts and three non-active galaxies, the stellar velocity curve rises regularly over the FOV (NGC 2655, 2985, 4051, 5055, 5194 and 5248). NGC 3227 can be classified in this category. Its stellar velocity curve keeps a value of about $65 \mathrm{~km} \mathrm{~s}^{-1}$ for $\mathrm{R}<10^{\prime \prime}$ ( $\sim 1 \mathrm{kpc}$ ) then rises continuously outwards.

2. For NGC 1068, the velocity curve presents a steep rise up to about $10^{\prime \prime}$ $(\sim 700 \mathrm{pc})$ and then remains constant between 180 and $200 \mathrm{~km} \mathrm{~s}^{-1}$ for larger radii.

3. For the Seyfert 1 galaxy NGC 4151, $V_{c}$ rises for $\mathrm{R}<11^{\prime \prime}(\sim 1 \mathrm{kpc})$ reaching a maximum value at $140 \mathrm{~km} \mathrm{~s}^{-1}$ and then decreases for larger radii.

4. In contrast, the stellar velocity curve of NGC 4579 decreases very smoothly for $\mathrm{R}<10^{\prime \prime}\left(\sim 820\right.$ pc)and rises steeply for $\mathrm{R}<15^{\prime \prime}$ corresponding to about $1.2 \mathrm{kpc}$. 
5. Finally, five galaxies (NGC 3351, 3627, 4459, 5806 and 6951) present more complicated rotation curves. For the two Seyfert galaxies NGC 3627 and 6951, the rotation curve may present a rise for $\mathrm{R}<160 \mathrm{pc}$ and $\mathrm{R}<600 \mathrm{pc}$, respectively. $V_{c}$ reaches a maximum at 80 and $169 \mathrm{~km} \mathrm{~s}^{-1}$ for NGC 3627 and 6951. A decline is observed up to $7^{\prime \prime}$ for these two galaxies, which corresponds to about 230 pc for NGC 3627 and 800 pc for NGC 6951. Then $V_{c}$ rises steeply for larger radii larger. The rotation curve of the three non-active galaxies NGC 3351, 4459 and 5806 present a rise in the inner $5^{\prime \prime}$, steep for NGC $3351(\mathrm{R}<200 \mathrm{pc})$ and $5806(\mathrm{R}<700 \mathrm{pc})$, less pronounced in the case of NGC $4459(\mathrm{R}<400 \mathrm{pc})$. Then $V_{c}$ decreases up to 470 pc, 800 pc and $1.1 \mathrm{kpc}$ for NGC 3351, 4459 and 5806, respectively, and rises again for larger radii.

The gaseous velocity curves present much more complex shapes than the stellar ones, with variations on small scales (wiggles). As for the stellar components, the maximum gaseous circular velocity seems not to be reached within the SAURON FOV for all the galaxies except three: NGC 4579, 5806 and 6951. In the case of NGC 4459, it seems that $V_{c}$ reaches a maximum and then declines, but since no more information is available, this cannot be confirmed. We can divide the gaseous rotation curves in three categories, depending on their behaviour in the innermost regions:

1. First the gaseous velocity curves decrease in the inner 5 to $10^{\prime \prime}$. This is the case for six Seyfert galaxies. This decline is very steep for NGC 3227 $\left(100 \mathrm{~km} \mathrm{~s}^{-1}\right.$ in $\left.3^{\prime \prime}=300 \mathrm{pc}\right)$ and probably for NGC $2655\left(50 \mathrm{~km} \mathrm{~s}^{-1}\right.$ in $2^{\prime \prime} \approx$ $240 \mathrm{pc})$. It is smoother in the case of NGC $4151\left(20 \mathrm{~km} \mathrm{~s}^{-1}\right.$ in $\sim 6^{\prime \prime}=590$ pc), $3627\left(15 \mathrm{~km} \mathrm{~s}^{-1}\right.$ in $\left.10^{\prime \prime}\right)$ and $5194\left(50 \mathrm{~km} \mathrm{~s}^{-1}\right.$ in $\left.8^{\prime \prime}=300 \mathrm{pc}\right)$. None non-active galaxies present such a decrease of $V_{c}$ in the more central regions. For larger radii, the rotation curve may present several different radial variations. It rises with wiggles in the cases of NGC 2655 and 5194, and declines with wiggles for NGC 3227. The gaseous velocity curve of 3627 rises regularly for $\mathrm{R}>13^{\prime \prime}\left(400 \mathrm{pc}\right.$ ) and in the case for NGC 1068, $V_{c}$ presents a steep rise for $\mathrm{R}>6^{\prime \prime}(420 \mathrm{pc})$ and then remains constant around $170 \mathrm{~km} \mathrm{~s}^{-1}$ for larger radii. The velocity curve of NGC 4151 remains also constant, around $180 \mathrm{~km} \mathrm{~s}^{-1}$ at all radii, with a wave shape of about 20 $\mathrm{km} \mathrm{s}^{-1}$ in amplitude.

2. The gaseous velocity curve can also present a rise in the inner regions, as for five non-active (NGC 2985, 3351, 5055, 5248, 5806) and two Seyfert galaxies (NGC 4579 and NGC 6952). Outwards, the shapes of $V_{c}$ can be very different. In the cases of NGC 5806, 5248, 3351, and 6951, after reaching a local velocity maximum, $V_{c}$ presents a decline and reaches a minimum velocity. It then experiences a second rise outwards. For NGC 2985, $V_{c}$ rises at all radii, with wiggles, and for NGC 4579, a maximum velocity of $350 \mathrm{~km} \mathrm{~s}^{-1}$ is reached at $13^{\prime \prime}$ (about $1 \mathrm{kpc}$ ), then a decline is observed. In the case of NGC 5055, ionised gas velocity curve remains constant around $170 \mathrm{~km} \mathrm{~s}^{-1}$ for radii larger than $12^{\prime \prime}=420 \mathrm{pc}$. 
3. Finally for two galaxies, the ionised gas velocity curve keeps a constant value in the innermost regions. A rise is observed for $\mathrm{R}>410 \mathrm{pc}$ and $\mathrm{R}>1.2$ kpc in the case of NGC 4459, and 4051, respectively. $V_{c}$ rises regularly in the case of NGC 4051, while for NGC 4459 it rises and reaches a maximum velocity $\left(300 \mathrm{~km} \mathrm{~s}^{-1}\right.$ ) at $8^{\prime \prime}=650 \mathrm{pc}$ and then seems to decline. However, this last feature observed in the gaseous rotational curve of NGC 4459 may be due to the low signal-to-noise of the velocity maps of this galaxy (see Fig. C.1b).

In general, the amplitudes of the gaseous velocity curves are higher than those of the stellar velocity curves. This is the case at all radii for eight of our galaxies, four Seyfert (NGC 2655, 4051, 4151 and 4579) and four non-active galaxies (NGC 4459, 5248, 2985 and 5055). For the remaining seven galaxies, six of them present higher gaseous $V_{c}$ than stellar ones in a part of the FOV (NGC 6951, 5194, 3627, 1068, 5806 and 3227), and the stellar and ionised gas velocity curves reach similar values in the other part. Finally, for NGC3351, gaseous velocity field is alternatively higher and lower than the stellar one. 

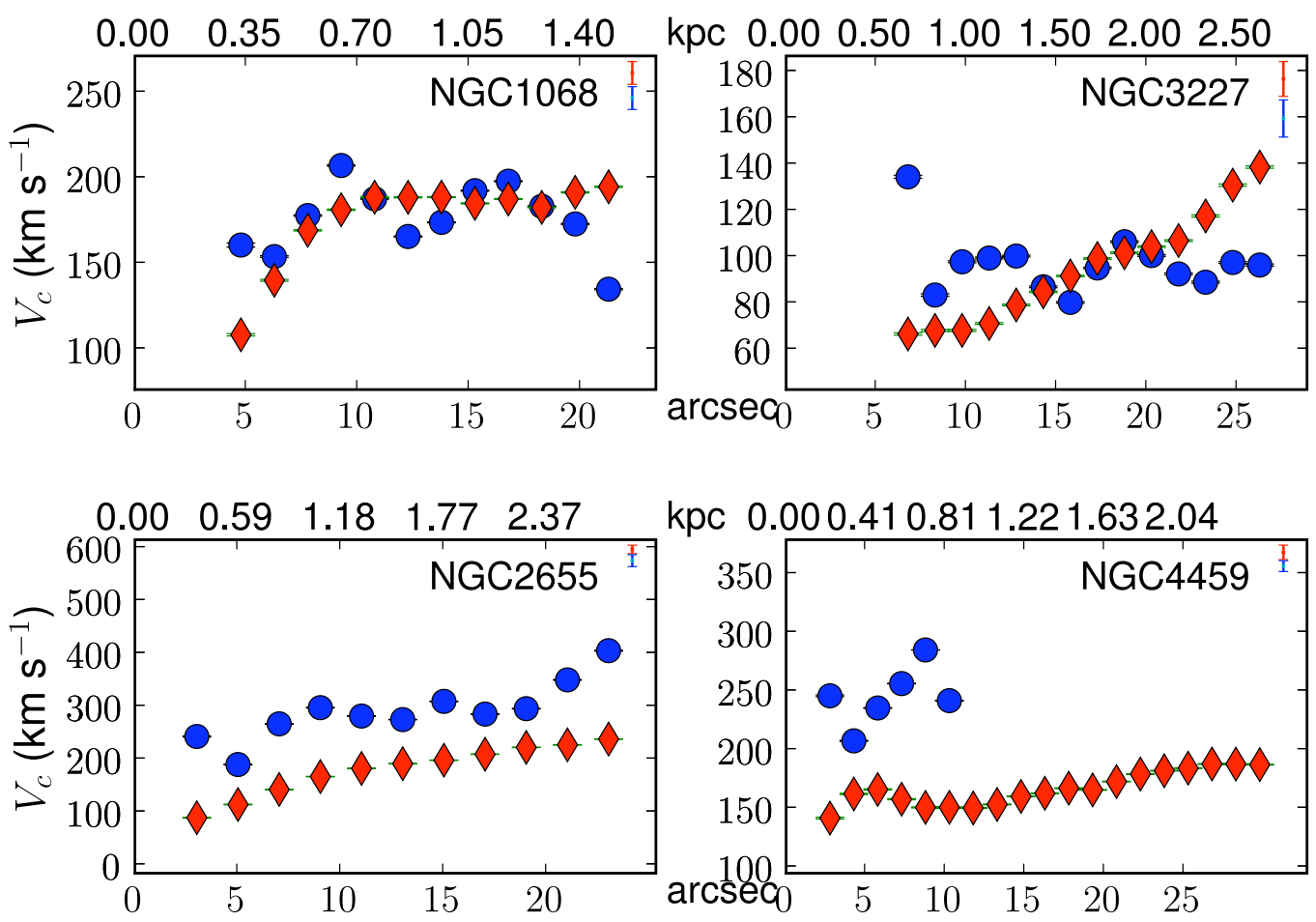

kpc 0.000 .410 .811 .221 .632 .04
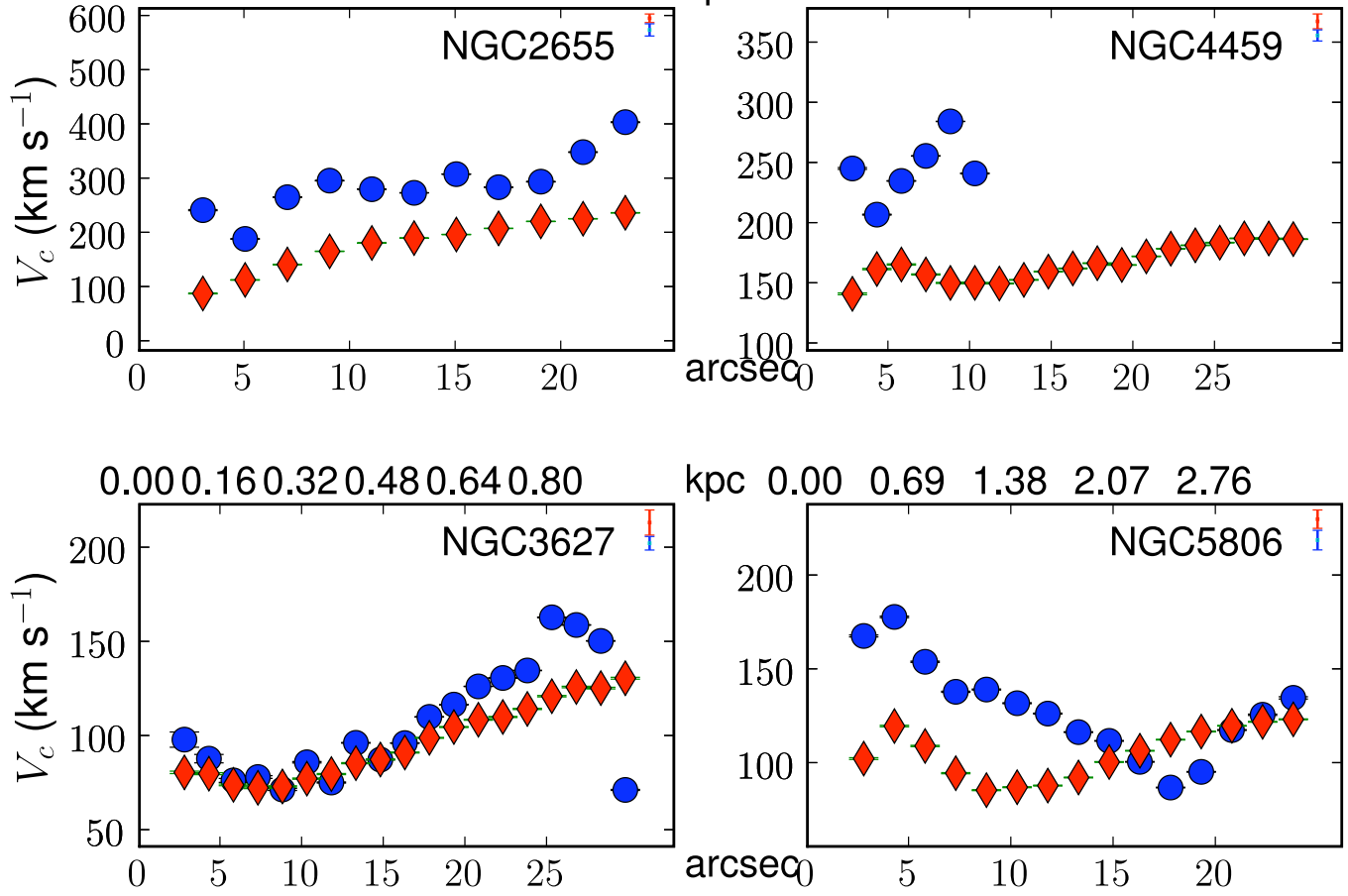

$\begin{array}{llllll}\text { kpc } & 0.00 & 0.69 & 1.38 & 2.07 & 2.76\end{array}$
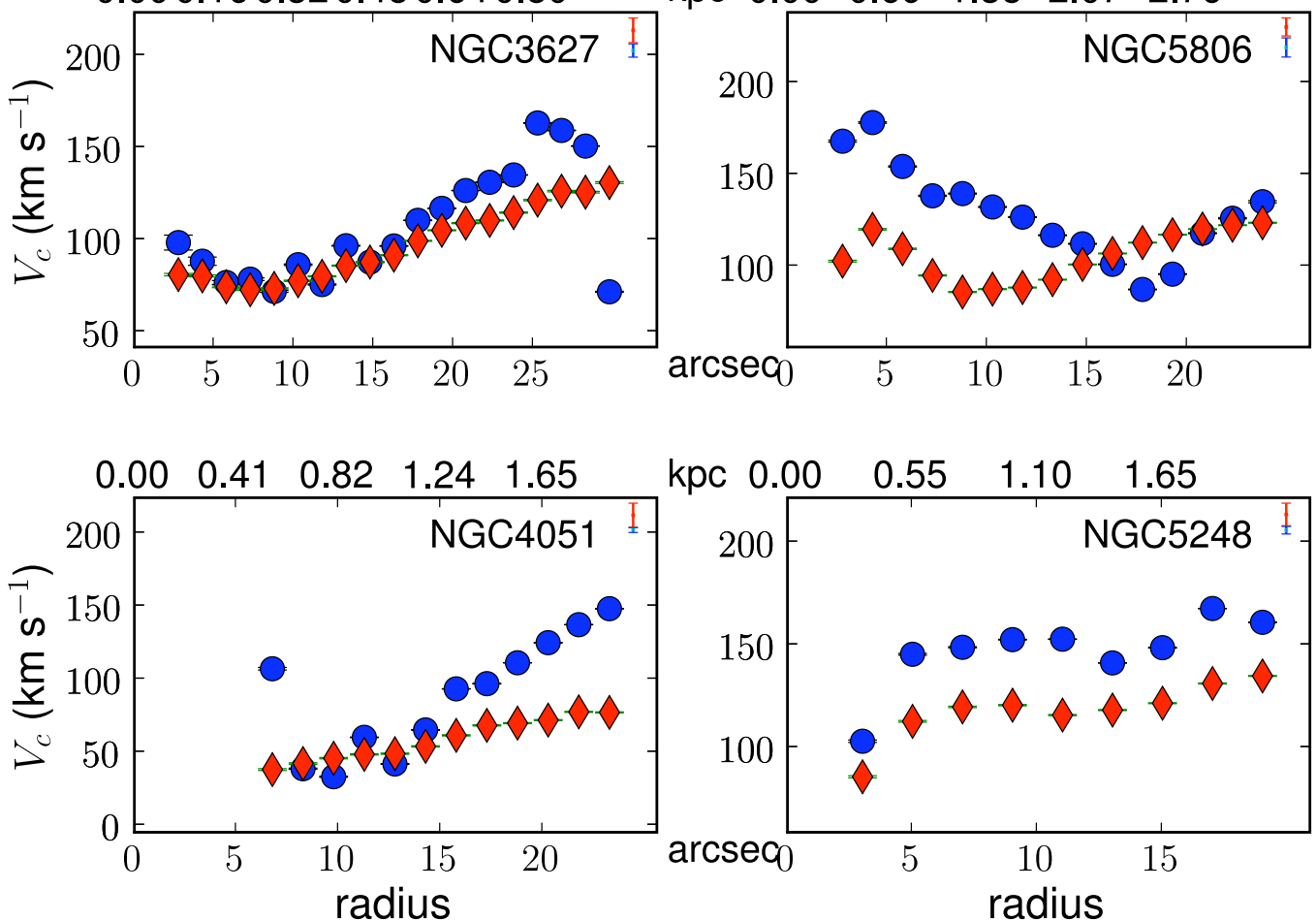

kpc $\quad 0.00 \quad 0.55 \quad 1.10 \quad 1.65$

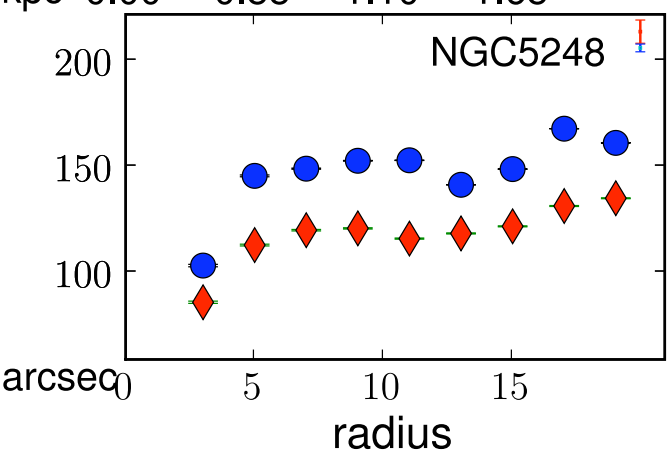

Figure 5.18a: Rotation curve as a function of radius of the stars (red diamonds) and of the gas (blue circles) components. The first row shows the Seyfert galaxies NGC 1068, and NGC 3227. Each subsequent row presents a pair of galaxies: the Seyfert on the left, and the inactive galaxy on the right. The errorbars of each point are smaller than the size of the symbol, and then are not visible. The red and blue error bars drawn in the top right corner of each plot represent 20 times the typical errorbar of the stellar and gaseous PAs, respectively. 


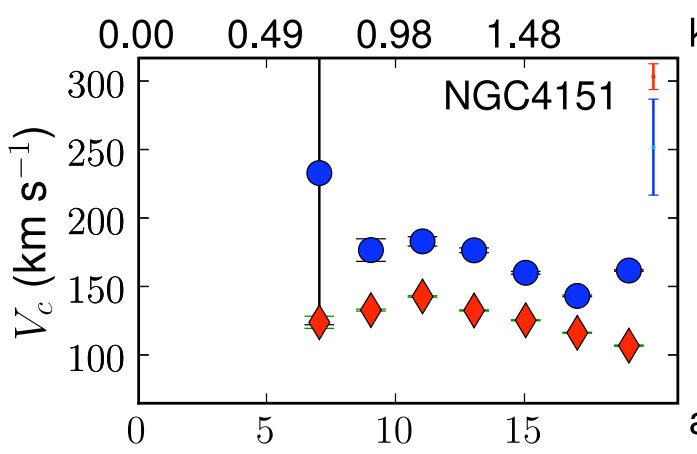

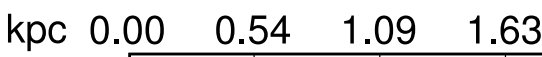
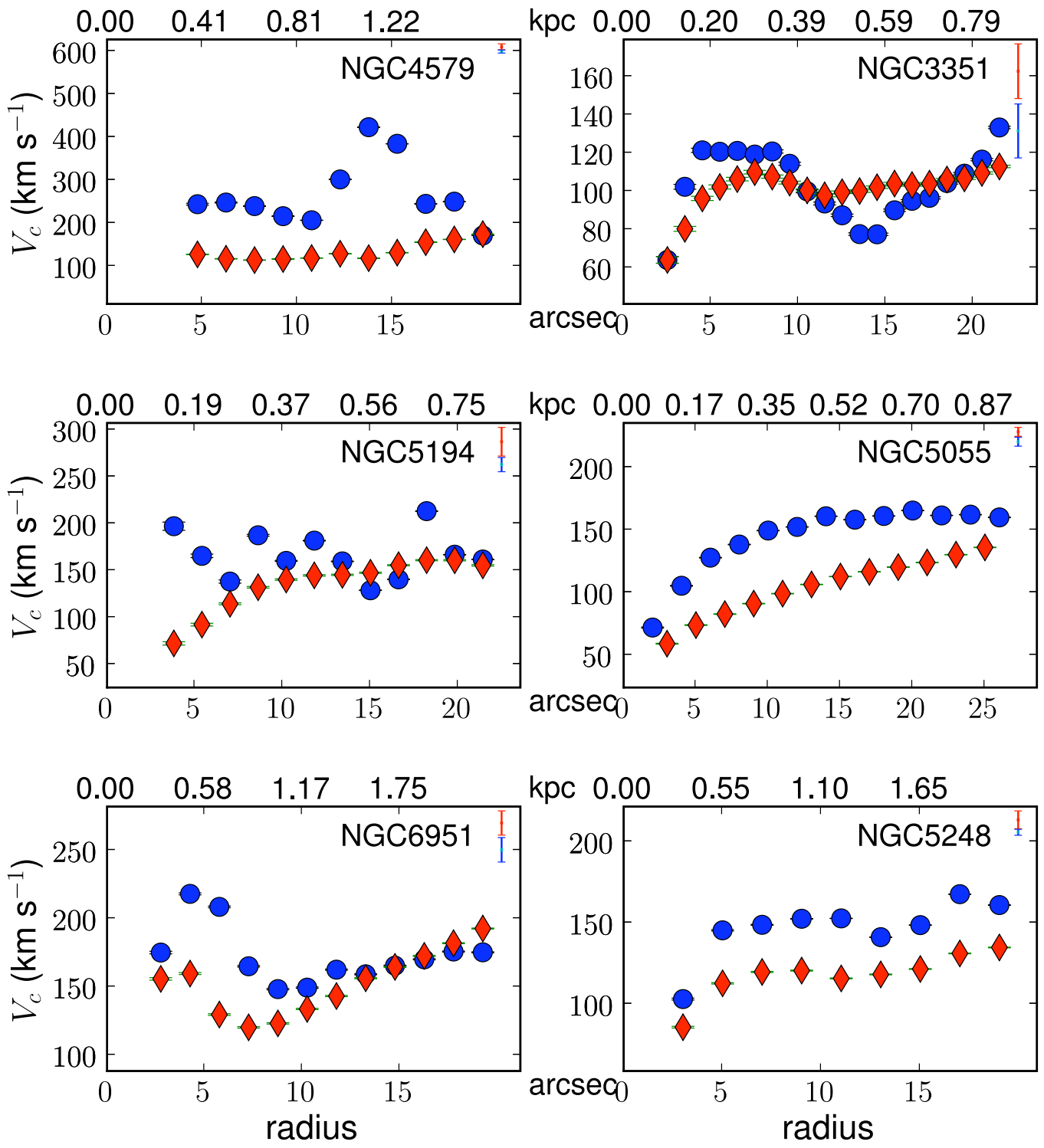

Figure 5.18b: Fig. 5.18a continued. 


\subsubsection{H I velocity curves and position angles.}

The PyRing tilted-ring fit was also performed on the H I velocity fields. H I data for four pairs have been reduced so far (NGC 3227 and 3169, NGC 4151 and 2985, NGC 4593 and 5383 and NGC 4941 and 3705). The velocity curves and radial variations of the H I PA are shown in Fig. 5.19a. Figs. 5.19b and 5.19c present the $\mathrm{H}$ I velocity curves and PA of the remaining five Seyfert and eight non-active galaxies, respectively.

\section{- Radial variations of the kinematic PA.}

For all the galaxies, the average $\mathrm{H}$ I kinematic PA over the radius are in good agreement with the orientation of the optical outer disk PA. In some cases the H I PA corresponds to the LON orientation only in the outer parts. This is the case for NGC 2655. As shown in Fig. 5.20, the orientation of the H I kinematic PA in the outer disk of this galaxy corresponds with the orientation of the stellar PA in the circumnuclear regions. Then the H I kinematic PA change orientation inwards, being in good agreement with the ionised gas PA in the innermost regions.

\section{- Rotation curves.}

In general, the $\mathrm{H}$ I rotation curves of our galaxies present a rise in the inner regions, except for NGC 2655, for which $V_{c}$ declines for $\mathrm{R}<14 \mathrm{kpc}$. Beyond the innermost regions, the velocity curves present complex shapes:

- For three Seyfert (NGC 3227, 4151, and 4593) and five non-active galaxies (NGC 2985, 4041, 5364, 5383 and 5806), $V_{c}$ declines after reaching a maximum velocity. For the latter four galaxies (NGC 4593, 5364, 5806), $V_{c}$ remains roughly constant at its maximum before declining for larger radii.

- The velocity curves rises continuously outwards in the case of five Seyfert (NGC 4051, 4639, 5364, 4941 and 6951) and three non-active galaxies (NGC 3351, 3705 and 5364). This rise is very regular for NGC 4639. It is steep in the inner regions, then smoother at larger radii for four of these galaxies (NGC 4051, 3351, 5364, 6951). For NGC 4941 and 3705, the velocity curve presents a more complex behaviour with successive rises and declines, still with an overall rise.

- The HI velocity curve may present also a complex behaviour. For NGC 2655, $V_{c}$ rises steeply for $\mathrm{R}>14 \mathrm{kpc}$, at which it reaches a minimum velocity of $50 \mathrm{~km} \mathrm{~s}^{-1}$. The velocity curve of NGC 4258 presents a very steep rise in the inner $3 \mathrm{kpc}$ reaching a maximum velocity of about 240 $\mathrm{km} \mathrm{s}^{-1}$. It then declines reaching a minimum of $160 \mathrm{~km} \mathrm{~s}^{-1}$ at $\mathrm{R} \sim 10 \mathrm{kpc}$, and rises smoothly for larger radii. NGC 3169 presents a similar rotation 
curve, with more wiggles. For the remaining three galaxies (NGC 2967, 3963, 4548), the velocity curves present several successive rises and declines after a first rise in the inner part. They reach a maximum velocity in the second rise, and rise in the outer part of the FOV. 

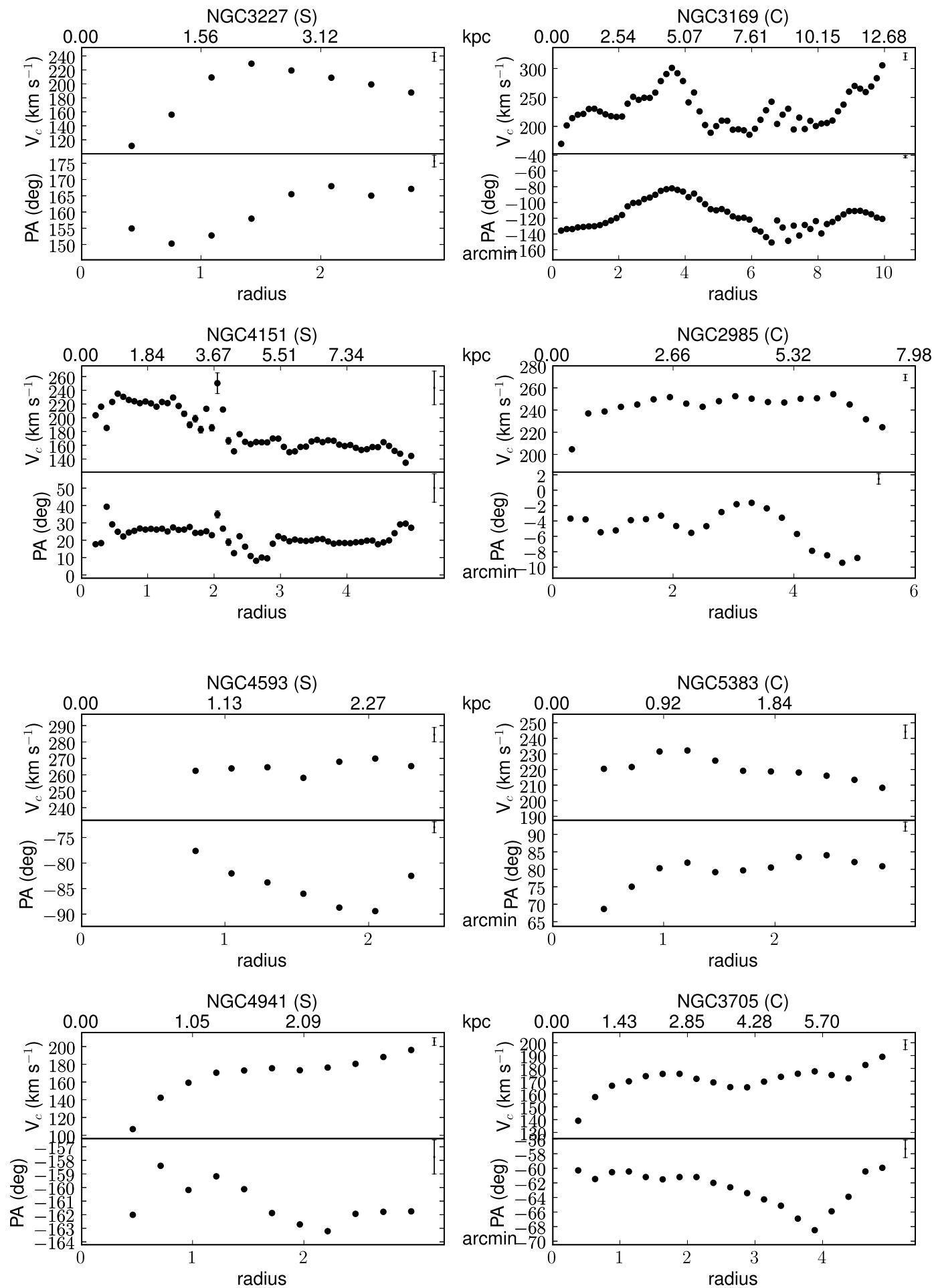

Figure 5.19a: Rotation curve (top panels) and kinematic position angles as a function of radius of neutral gas components. The ' $S$ ' and ' $C^{\prime}$ on the right of the galaxy name stand for Seyfert or Control galaxy, respectively. In each rows, the Seyfert galaxy is shown on the left, and its associated inactive galaxy on the right for the four pairs with $\mathrm{H}$ I maps available at this time. The errorbars of each point are smaller than the size of the symbol, and then are not visible. The black error bars drawn in the top right corner of each plot represent 50 times the typical errorbar of the PAs and circular velocity. 

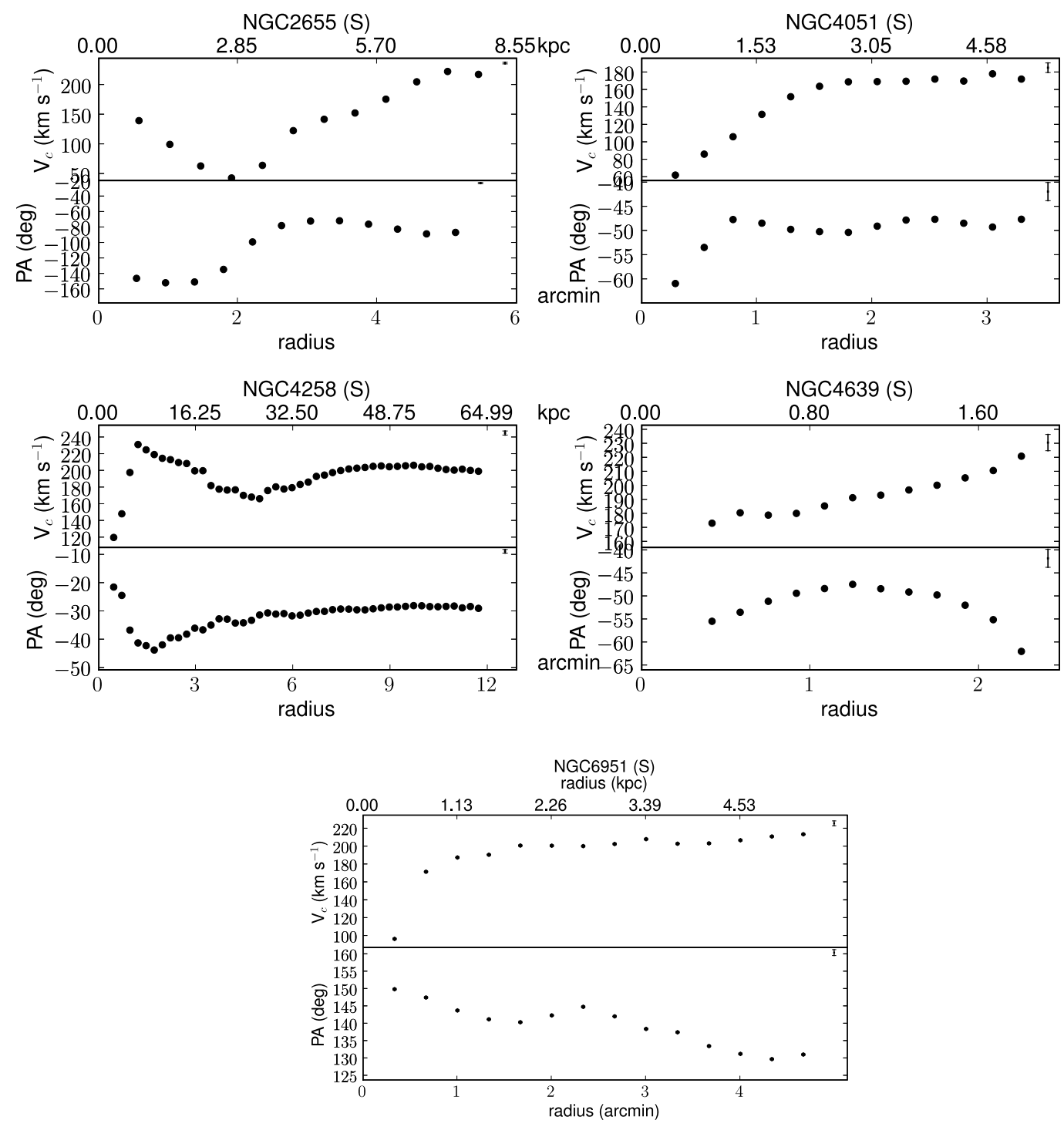

Figure 5.19b: Rotation curve and kinematic position angles of the neutral gas components for the remaining five Seyfert galaxies. 

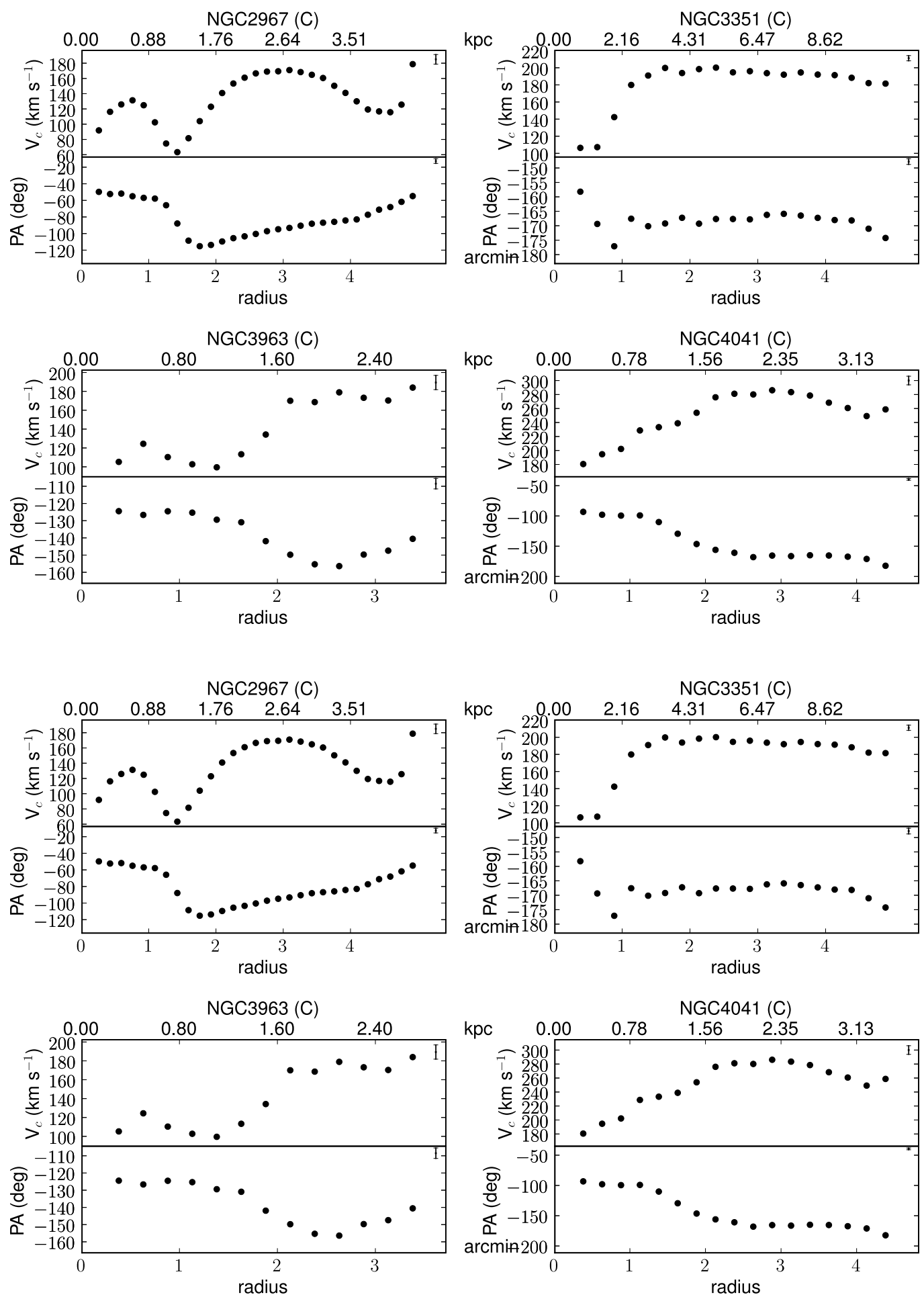

Figure 5.19c: Rotation curve and kinematic position angles of the neutral gas components for the remaining eight non active galaxies. 

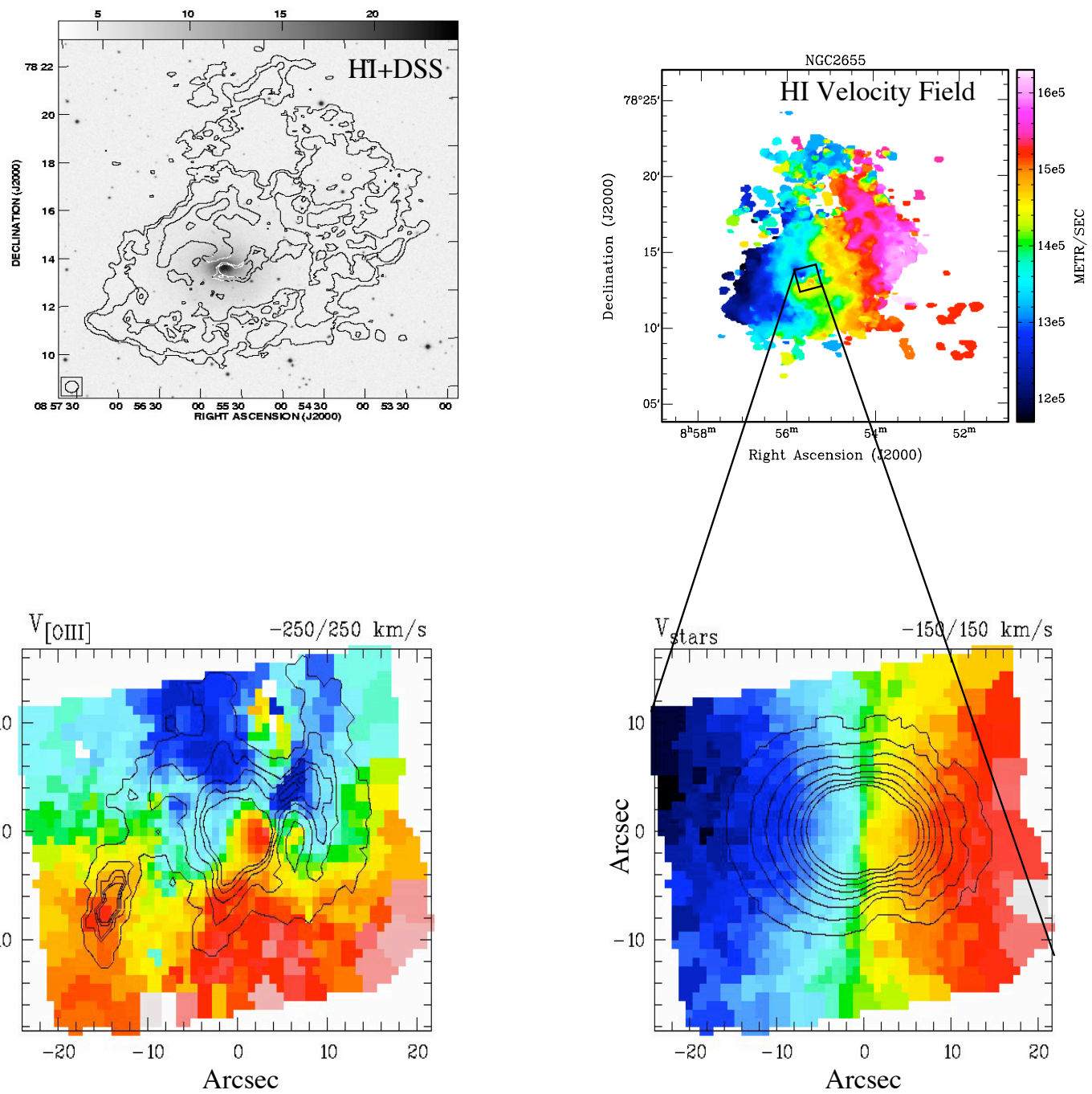

Ionised Gas Velocity Field

Stellar Velocity Field

Figure 5.20: Comparison of H I stellar and ionised gas kinematics of NGC 2655. The HI distribution (in contour on the DSS image) and velocity fields are shown in the top left and right panels, respectively. The black square overlaid on the Hi velocity maps correspond to the SAURON FOV. The SAURON gaseous and stellar velocity fields are presented in the bottom left and right panels, respectively. 


\subsubsection{Global variation of the kinematic parameters.}

Five Seyfert (NGC 2655, 3227, 4051, 4151, 6951) and four non-active galaxies (NGC 2985, 3351, 5055, 5806) of the master sample have available optical and radio data. Comparison of the kinematic in the central (optical data) and outer regions (radio data) is then possible. Figs. 5.21a and 5.21b present the velocity curves (top panels) and the kinematic PA (bottom panels) of the stars (red diamonds), ionised gas (blue circles) and neutral gas (black squares) for these nine galaxies, in semi-log plots, in order to emphasise the overlap regions of the H I and SAURON data.

We notice that for all of the four non-active galaxies (Fig. 5.21b), the ionised gas and neutral gas velocity curves present a good continuity at radii where the SAURON and VLA FOV overlap. For NGC 2985 and 5055 the stellar velocity curves is well below the gaseous ones. This shift is less obvious for the over two non-active galaxies. For the five Seyfert galaxies (Fig. 5.21a) the situation is more complicated. The overlap between ionised and neutral gas velocity curves is less clear. For NGC 4151, the continuity between H I and ionised gas velocity curves is very likely, with an obvious shift between stellar and gaseous velocities. Surprisingly, in the case of NGC 2655 and 4051, it seems that the stellar velocity curve is the continuity of the $\mathrm{H}$ I one in the inner part, with the ionised gas velocity curves well above.

Regarding the radial variations of the kinematic PAs, the stellar and $\mathrm{HI}$ kinematic PAs are in good agreement for most of the galaxies (NGC 3227, 4151, 4051, 2985, 3351, 5055, and 5806). For NGC 2655, the stellar kinematic PA corresponds to the $\mathrm{HI}$ PA at radii larger than $20 \mathrm{kpc}$ while in the inner regions, the H I PA reaches similar values than the ionised gas kinematic PA. 
NGC2655

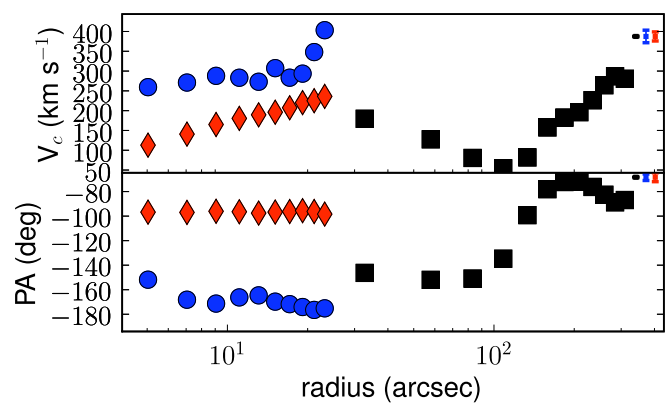

NGC4051

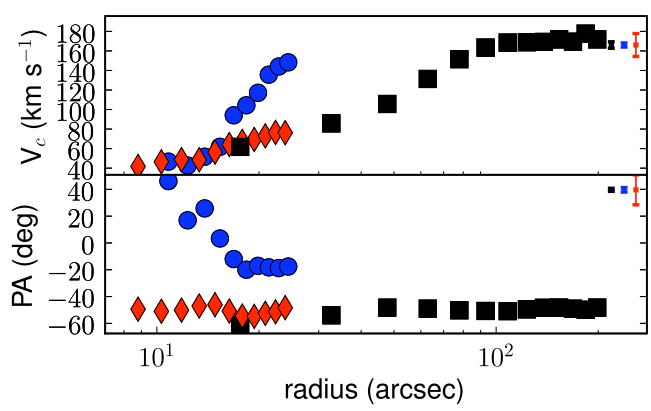

NGC3227

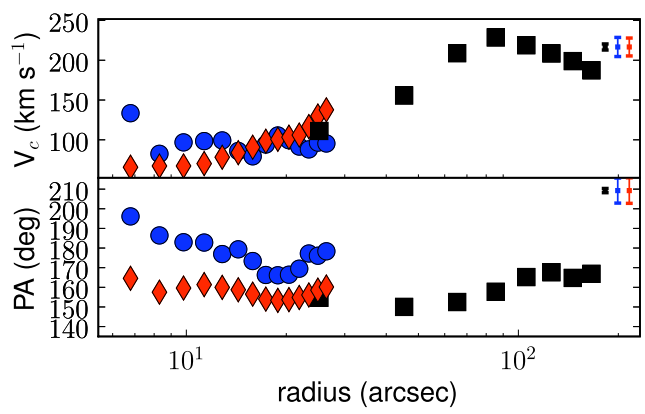

NGC4151

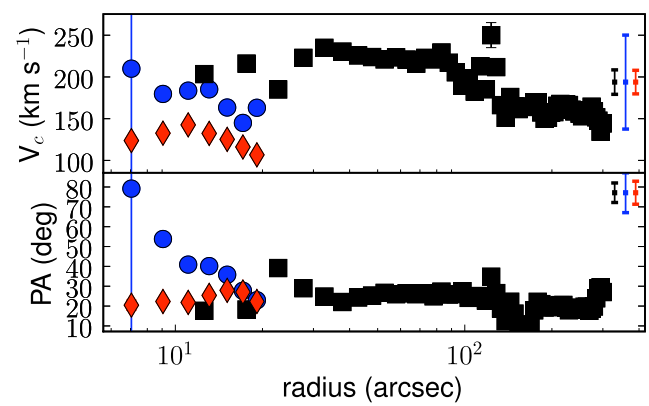

NGC6951

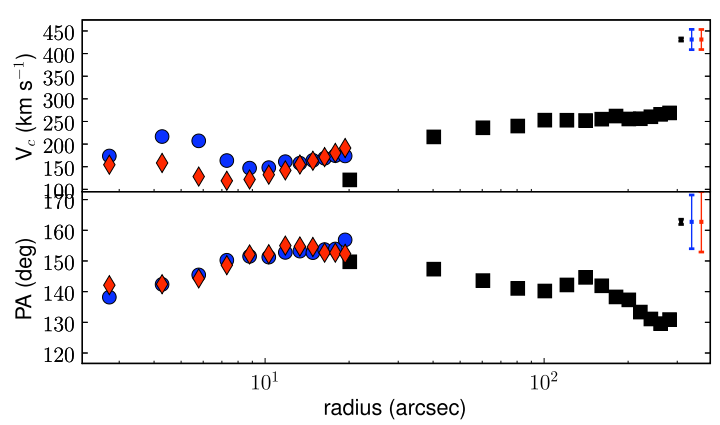

Figure 5.21a: Rotation curve (top panels) and kinematic position angles (bottom panels) as a function of radius of neutral gas (black squares), ionised gas (blue circles) and stellar components (red diamonds), for the Seyfert galaxies with available SAURON and H I maps. The red, blue and black error bars drawn in the top right corner of each panel represent 30 times the typical errorbar of the circular velocity (top panels) and the PAs (bottom panels) for the stars, ionised gas and atomic gas, respectively. 
NGC2985

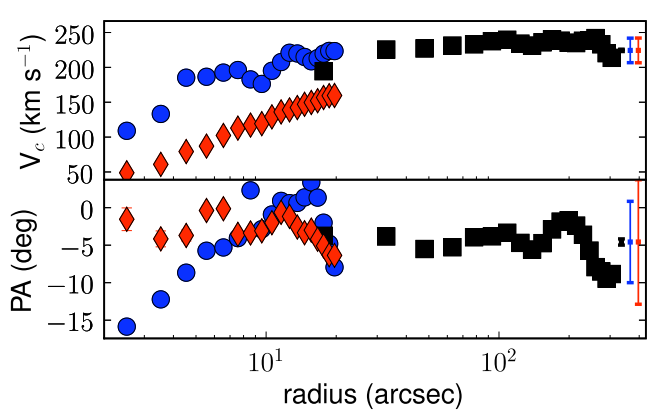

NGC5055

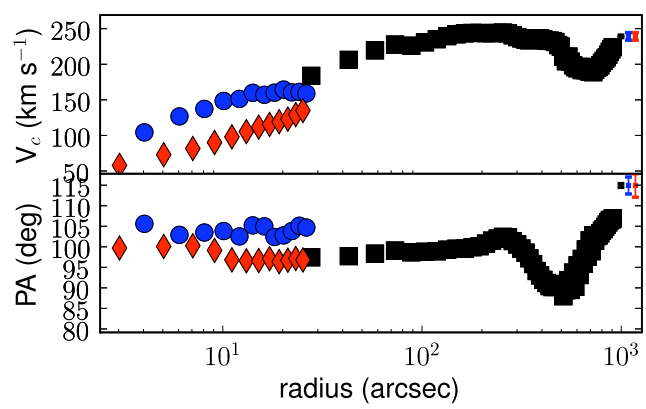

NGC3351

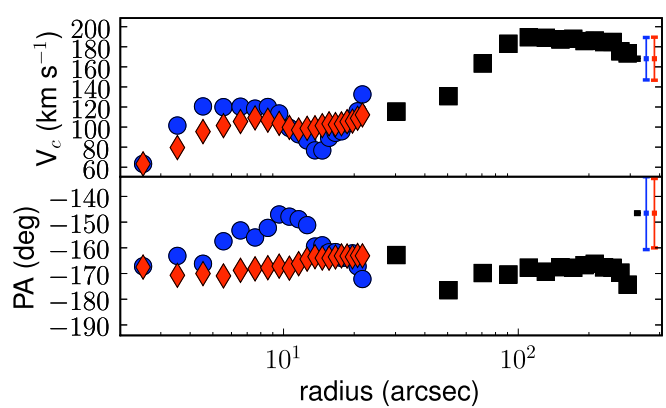

NGC5806

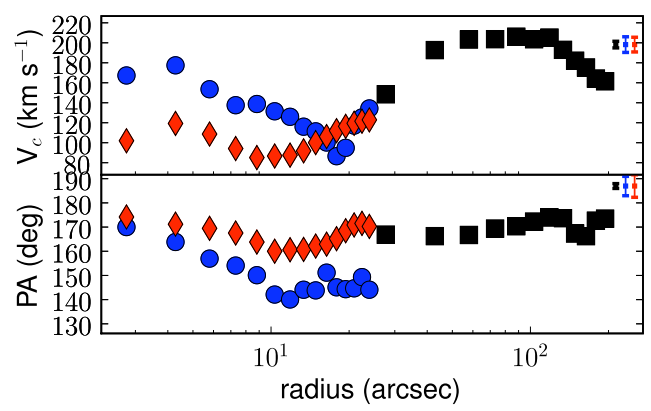

Figure 5.21b: Rotation curve (top panels) and kinematic position angles as a function of radius of neutral gas (black squares), ionised gas (blue circles) and stellar components (red diamonds), for the non-active galaxies with available SAURON and HI maps. The red, blue and black error bars drawn in the top right corner of each panel represent 30 times the typical errorbar of the circular velocity (top panels) and the PAs (bottom panels) for the stars, ionised gas and atomic gas, respectively. 


\subsection{Summary and main results}

In this chapter we have investigated the two-dimensional kinematics of the Seyfert and non-active galaxies, in the large-scale H I disk and in the circumnuclear regions, via the stellar and gaseous components. We first determined the global orientation of the kinematic major-axis of the stars and ionised gas, in the central regions. We found that:

- The stellar global kinematic major-axes are aligned within $10^{\circ}$ with the outer galactic disk major-axes for all the galaxies of the SAURON/Seyfert sample, except for two Seyfert 2 galaxies: NGC 1068 and NGC 5194, for which the determination of the photometric major-axis is quite uncertain. Therefore, except for these two particular galaxies, the circumnuclear stellar kinematic major-axis is a robust determination of the line-ofnodes for both Seyfert and non-active galaxies.

- The ionised gas co-rotates with the stars, the global stellar stellar and gaseous PAs being aligned for all the galaxies observed with SAURON within $20^{\circ}$, except for two Seyferts: NGC 2655 and NGC 4579.

We then focused on the radial variations of the kinematic parameters. We developed the PyRing tool, using the tilted-ring method to derive the parameters of the H I stellar and ionised gas velocity fields. We compared this tool with the AIPS task GAL and the GIPSY routine ROTCUR, and we showed that PyRing can fit robustly the kinematic and geometrical parameters of our twodimensional kinematic maps. We performed then a first fit, assuming purely rotation motions in a thin disk, in order to derive the velocity curves and the kinematic PAs as a function of radius for the stars, ionised gas and neutral gas. The results of this analysis are:

- In the circumnuclear regions, the gaseous kinematic PAs have radial variations $\sim 3.5$ times greater than the stellar ones, for the Seyfert. In the case of the inactive galaxies, the radial variations of the ionised gas PAs are 2 times greater than the stellar ones.

- The variations of the stellar PAs in the central kpc of the Seyfert galaxies are 2 times greater than those of the non-active galaxies, while those of the ionised gas PAs are $\sim 3$ times greater for the Seyferts than for the corresponding non-active galaxies.

- Regarding the large-scale disk, for all galaxies with H I data, the largescale H I kinematic PAs correspond to the LON orientations and indeed for the galaxies both in the VHIKINGS and SAURON/Seyfert surveys, the outer H I PA and the circumnuclear stellar PA are in good agreement with each other. 
- In the circumnuclear regions, the gaseous velocity curves reach higher values than the stellar ones for the majority of our galaxies. In general, the stellar component presents more regular velocity curves than the ionised gas, and the stellar maximum velocity is not reached within the SAURON FOV, except for the Seyfert 1 galaxy NGC 4151. The gaseous velocity curve reaches its maximum velocity for three galaxies NGC 4579, 5806 and 6951.

- The H I rotation curves are overall regular. They all rise in the innermost regions, except for NGC 2655.

- Comparison of the rotation curve shapes between the large-scale disk and the circumnuclear regions has been done for 9 of our galaxies. For all four non-active galaxies and three Seyferts there is a good continuity between the large-scale and circumnuclear kinematic information. The situation is less clear for the remaining two Seyferts NGC 2655 and NGC 4051. 


\section{Chapter 6}

\section{Discussion and Perspectives}

In this thesis, we presented the first results of the VHIKINGS and SAURON/Seyfert surveys. These two surveys aim at a comprehensive comparative study of the distribution and kinematics of neutral gas in the large-scale disk, and stars and ionised gas in the circumnuclear regions of Seyfert and inactive galaxies.

Moment maps have been derived for all the galaxies of the SAURON/Seyfert survey and for 21 galaxies of the VHIKINGS survey (Chap. 4) and a preliminary kinematic analysis has been conducted in Chap. 5, using our PyRing tilted-ring tool, dedicated to our multiwavelengths and multi-scale data. In this chapter, I discuss the results of this study in the context of AGN fuelling.

\subsection{Feeding the AGN and time-scales.}

In this study, we investigated the stellar and ionised gas kinematics of a welldefined sample of nearby active and inactive galaxies, in order to probe the galactic potential in the central kpc regions. In Sec. 4.1.1, we described the SAURON stellar distribution maps. Five Seyfert 2 galaxies present disturbed stellar morphology in the circumnuclear regions (NGC 1068, 2655, 3627, 4579 and 5194). Three of these galaxies (NGC 2655, 4579 and 5194) show strong kinematic misalignments between the stellar and gaseous components. For the remaining two galaxies (NGC 1068 and 3627), gas is globally co-rotating with the stars. However, the stellar velocity fields of these galaxies present complex features (Sec. 4.1.2) with S-shaped zero-velocity curves or twisted major-axes, whereas all the others galaxies present overall regular stellar velocity fields. The only other galaxy which presents complex stellar morphology is the control galaxy NGC 5055. For this galaxy, the perturbation in the stellar isophotes is certainly due to dust absorption. This contrasts with the five Seyfert galaxies (NGC 1068, 2655, 3627, 4579 and 5194) for which stellar morphology and stellar or gaseous kinematic perturbations seem to be intimately related. This result is in good agreement with the study of Hunt \& Malkan (2004). They 
found an excess of isophotal twists in the circumnuclear regions of Seyfert 2 galaxies relative to normal and Seyfert 1 galaxies.

The distribution of kinematic misalignments has often been used to constrain the origin of the ionised gas in early-type galaxies (Bertola et al., 1992; Sarzi et al., 2006). If the gas is produced internally (e.g. stellar mass loss) and comes from the galactic disc, the bulk of the gaseous component in the central region is expected to co-rotate with the stars. The angular momenta of the stellar and gaseous components would therefore be globally aligned. Some mild misalignments may exist due to the effect of non-axisymmetric structures (bars, spirals), or the presence of decoupled stellar components. Gas acquired from an external source, e.g. via interaction with galaxies or the intergalactic medium, should eventually settle in the existing principal planes of the gravitational potential. In a triaxial potential hosting a large-scale disc, the gas would thus preferentially fall onto either the disc equatorial plane or in a plane perpendicular to it, giving rise to a polar ring. Polar rings are observed in a few nearby early-type galaxies (see e.g. Bertola et al., 1992). The distribution of kinematic misalignments, measured as position angle differences between kinematic major-axes, around the reference values of 0,90 and $180^{\circ}$, should therefore be closely linked with the origin of the accreted gas.

So far, there seems to be no trend for the kinematic misalignments of earlytype galaxies with respect to Hubble type, galactic environment and galaxy luminosity (Sarzi et al., 2006). In our sample, five Seyfert galaxies (NGC 1068, $2655,3227,4579,5195)$ present significant kinematic misalignments $\left(>20^{\circ}\right)$. For all the other Seyferts (NGC 3627, 4051, 4151, 6951) and all the inactive galaxies, the ionised gas co-rotates with the stars (kinematic misalignments smaller than $20^{\circ}$ ). This follows the results of Sarzi et al. (2006) for a sample of latertype galaxies and therefore suggests that the presently observed ionised gas in the circumnuclear regions does not have a purely external origin for both the Seyfert and the inactive galaxies. The only clear exception is NGC 2655, a galaxy known to host a polar ring (Keel \& Hummel, 1988). It is interacting with a companion and had presumably suffered a minor merger in the past (Huchtmeier \& Richter, 1982). Interactions between galaxies is an efficient way to transport gas towards the central parts and to trigger nuclear star formation (Barnes \& Hernquist, 1991), but it seems neither a necessary nor a sufficient mechanism for most Seyfert activity (De Robertis et al., 1998; Schmitt, 2001; Ho et al., 2003).

A more refined analysis of our sample revealed that the radial variations of the kinematic PAs of the ionised gas are three times greater for the Seyfert than for their associated inactive galaxies, in the central $1.5 \mathrm{kpc}$. Although our sample is small, this trend hints for the presence of non-axisymmetric perturbations of the gravitational potential in the inner kpc of the Seyfert galaxies. Since the observed stellar velocity fields of these active galaxies are very regular with major axes rather well aligned with the outer photometric ones, theses dynamical perturbations must be small. The gas, being more sensitive to small deviations from axisymmetry, responds better to non-axisymmetric structures 
such as weak bars or spirals. This would be in fact in agreement with previous imaging studies which found no strong excess of bars or nuclear spirals in Seyfert galaxies. If strong deviations from axial symmetric were present, the gaseous kinematics would have been extremely disturbed and easily detectable with standard (long-slit) spectroscopic techniques. The presence of weak deviations from axial symmetry in Seyfert galaxies seems to be in good agreement with the refined imaging study of Hunt \& Malkan (2004). They found an excess of NIR isophotal twists in Seyfert 2 hosts compared to inactive galaxies, and discussed the non-axisymmetric instabilities (nuclear disc, nested misaligned bars, triaxial structures) which could be responsible for such morphological perturbations. This excess of isophotal twist is not observed for the Seyfert 1 galaxies. Hunt \& Malkan (2004) interpreted this difference between Seyfert 1 and Seyfert 2 galaxies as an evolutionary effect, the Seyfert 1 hosts being 'older' than the Seyfert 2 ones in their scenario. We would need a larger sample to investigate statistical differences in the ionised gas properties of Seyfert 1 and 2 galaxies. A larger study of active vs. inactive galaxies in the SDSS with IMACS is now in progress within our group, the active and inactive galaxies drawn from the same parent sample, thus hopefully reducing any underlying selection biases and also offering more controls per Seyfert (Westoby et al. in preparation).

In addition to a ready supply of fuel and a transportation mechanism, timescales are important. Galaxies are thought to experience recurrent episodes of nuclear activity in their lifetime, with instabilities in the fuelling rate possibly regulating the activity cycles (Saripalli \& Mack, 2007). Dynamical instabilities in the inner kpc regions and nuclear activity can evolve in parallel. However, due to the time required to set up sufficient gas inflow, the nonaxisymmetric perturbations may be observed while not enough gas has been driven in the vicinity of the SMBH and the galaxy is inactive. Similarly such gravitational instabilities may have disappeared when the nuclear activity is still underway. Observationally, it is therefore reasonable to hypothesise that a snapshot of active and inactive galaxies might show equal degrees of host galaxy disturbance. However, while disturbed gas observed in a host galaxy is not the gas that is simultaneously fuelling the nuclear activity, the dynamical time scale in the circumnuclear regions is similar to the AGN lifetime. Therefore, the correlation between the presence of perturbation in the ionised gas in the central $\mathrm{kpc}$ regions and the nuclear activity suggested by our study is striking and supports a close link between the AGN, host dynamics on scales of hundreds to tens of parsecs and relevant dynamical and activity timescales.

Another dynamical signature of past accretion of gas in the central regions can be found in the stellar kinematics: central low stellar velocity dispersions or the so-called $\sigma$-drops. Wozniak et al. (2003) interpreted such structures as the result of star formation following the accretion of gas in the central few hundred parsecs. More recently, Wozniak \& Champavert (2006) showed that these dynamical structures can be formed on relatively short timescales $(\sim 300$ Myr) and that a star formation rate as low as $1 \mathrm{M}_{\odot} \mathrm{yr}^{-1}$ in the nuclear regions is enough to sustain a long-lived $\sigma$-drop. 


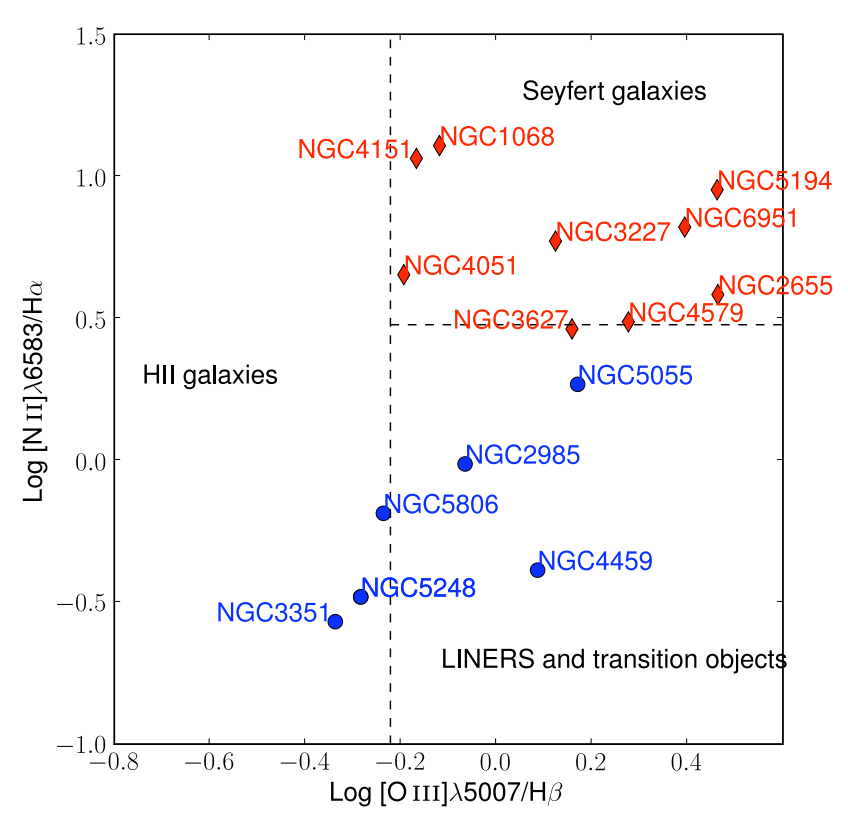

Figure 6.1: Diagnostic diagram for the galaxies of our sample, $\log ([\mathrm{O} I I I] \lambda 5007 / \mathrm{H} \beta)$ vs $\log ([\mathrm{N}$ II $] \lambda 6583 / \mathrm{H} \alpha)$. The emission line ratios are from Ho et al. (1997). Active galaxies are shown as filled red diamonds and inactive galaxies as filled blue circles. The classification boundaries from Ho et al. (1997) are shown as black dashed lines.

Such $\sigma$-drops seem to be very common in nearby spiral galaxies and the list of observed $\sigma$-drops is growing (e.g. Emsellem et al., 2001; Márquez et al., 2003; Ganda et al., 2006; Emsellem, 2006). At least one third of spiral galaxies are expected to host a $\sigma$-drop (Ganda et al., 2006). Most of the galaxies with a $\sigma$-drop are active nuclei, but this is certainly an observational bias since they are wellstudied and luminous galaxies, where reliable stellar kinematics has been obtained. In our sample, $\sigma$-drops are observed in five Seyfert galaxies (NGC 1068, $2655,3227,4051,4151,6951$ ) and one non-active galaxy (NGC 5248). High resolution follow-up IFU mapping of the central few arcseconds, using e.g. the OASIS or FLAMES IFU, could reveal direct evidence for these discs as shown by Emsellem (2006). Such data on a relatively small FOV are rather difficult to interpret. However, these data, associated with our larger scale SAURON maps, could extend our understanding of fuel delivery closer to the AGN.

All the dynamical structures discussed above (e.g. kinematic misalignments, $\sigma$-drops) may probe accretion of material in the central $100 \mathrm{pc}$ or so. However, studying such structures do not carry direct information on how the gas is funnelled inwards, within the parsec and sub-parsec scales nuclear regions. 

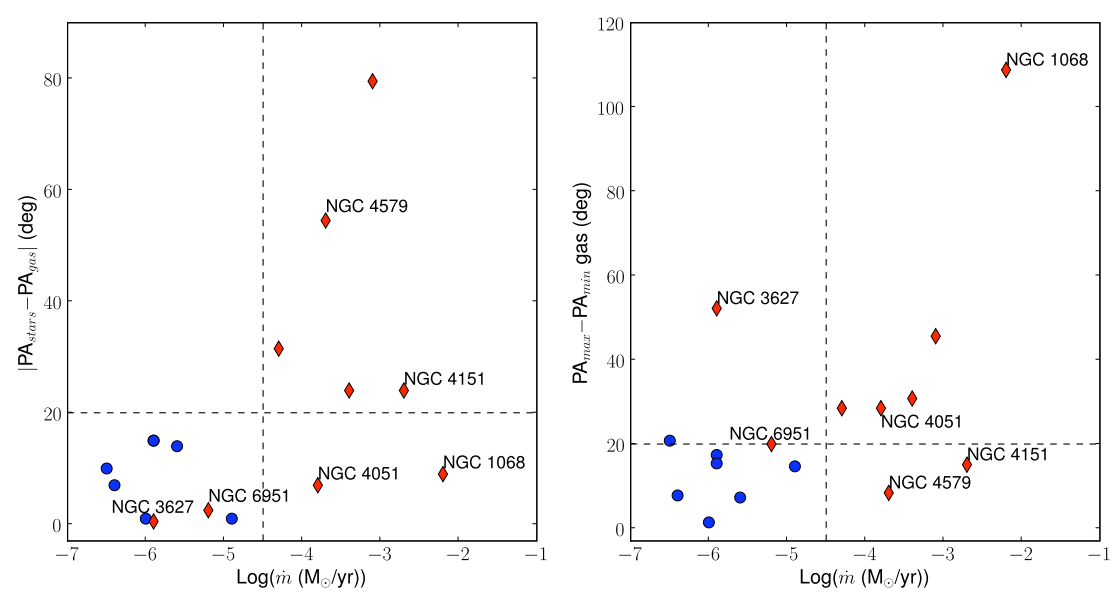

Figure 6.2: Distribution of the differences between the global kinematic PAs of the stars and gas (left panel) and the amplitude of variations of the gaseous kinematic PAs in the inner $1.5 \mathrm{kpc}$ (right panel) as a function of the mass accretion rate (see Table 6.1). Active galaxies are shown as filled red diamonds and inactive galaxies as filled blue circles. For reference, a line is drawn at $\triangle \mathrm{PA}=20^{\circ}$ and mass accretion rate $=10^{-4.5} \mathrm{M}_{\odot} / \mathrm{yr}$ in each of the panels. NGC 3627 and 6951 are the only Seyfert galaxies with accretion rate lower than $10^{-4.5} \mathrm{M}_{\odot} / \mathrm{yr}$. The other galaxies named in these plots correspond to the Seyferts which do not follow the same trend in the first and second plot (see text). 


\begin{tabular}{|c|c|c|c|c|c|c|c|c|}
\hline 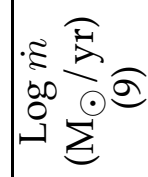 & 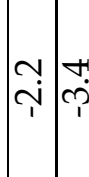 & 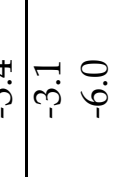 & 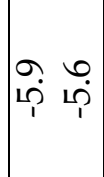 & $\begin{array}{ll}\infty & \sigma \\
\dot{p} & \stackrel{p}{p}\end{array}$ & 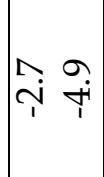 & $\begin{array}{ll}\wedge & 0 \\
0 & 0 \\
p & 0\end{array}$ & 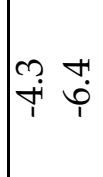 & 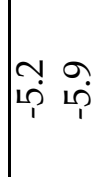 \\
\hline 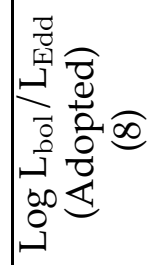 & 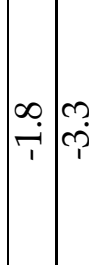 & 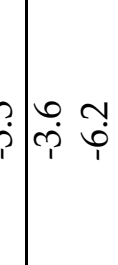 & 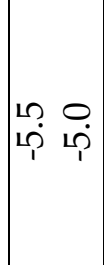 & 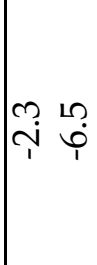 & $\left|\begin{array}{cc}N & 0 \\
& \stackrel{0}{*}\end{array}\right|$ & $\begin{array}{ll}\infty & 0 \\
p & 0 \\
p & 1\end{array}$ & $\mid \begin{array}{cc}0 & \infty \\
\dot{p} & \dot{p} \\
& \dot{p}\end{array}$ & $\mid$ \\
\hline 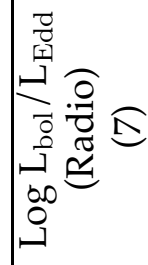 & 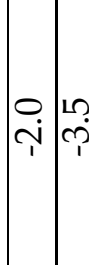 & 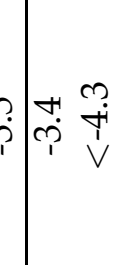 & iि. & $\begin{array}{l}\vec{H} \\
\stackrel{i}{i} \\
\stackrel{v}{v}\end{array}$ & $\left|\begin{array}{cc}0 & 1 \\
i & p \\
r & \dot{v}\end{array}\right|$ & $\underset{c}{\sim}:$ & $\mid \begin{array}{ll}\wedge & \sim \\
\infty & + \\
\dot{V} & \dot{v}\end{array}$ & $\begin{array}{l}\hat{\tilde{v}} \\
\hat{v} \\
v\end{array}$ \\
\hline 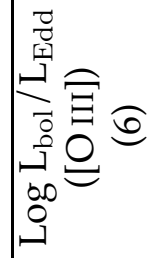 & 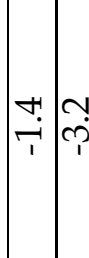 & \begin{tabular}{l|ll} 
& $\infty$ \\
\end{tabular} & 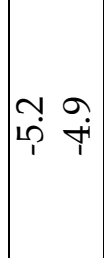 & $\begin{array}{ll}+ & 0 \\
i & \stackrel{0}{0}\end{array}$ & 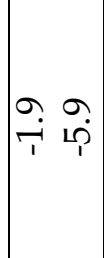 & 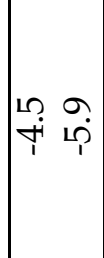 & $\hat{\widehat{N}}$ & $\mid$ \\
\hline 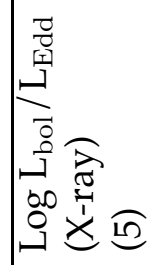 & ק. & 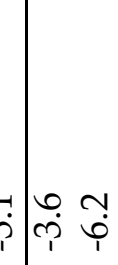 & $\begin{array}{l}0 \\
\dot{\varphi} \\
\dot{\varphi} \\
\dot{v}\end{array}$ & $\vec{i}$ & î & $\stackrel{\circ}{+}$ & $\begin{array}{ll}\wedge & 0 \\
& \dot{\varphi}\end{array}$ & Pיं \\
\hline 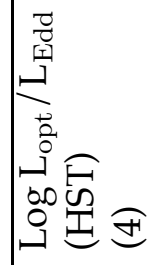 & & & & ๙o & $\overrightarrow{\hat{i}}$ & & & \\
\hline 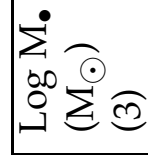 & 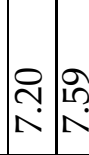 & 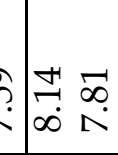 & 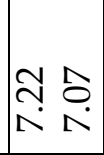 & 코 & $\begin{array}{ll}\infty & 0 \\
& \stackrel{1}{n} \\
\end{array}$ & 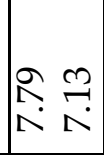 & 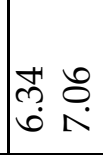 & 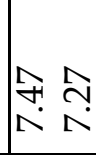 \\
\hline 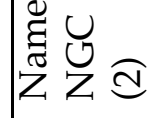 & 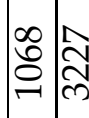 & 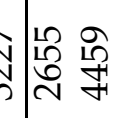 & 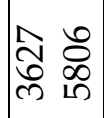 & 롱 & 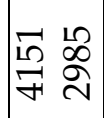 & 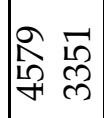 & 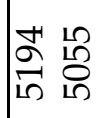 & 롱 \\
\hline 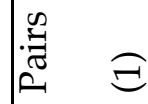 & & - & $N$ & $m$ & H & Ln & 6 & $\Lambda$ \\
\hline
\end{tabular}

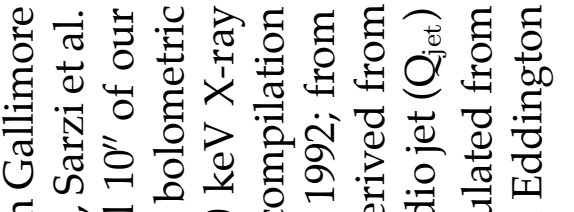
द्व

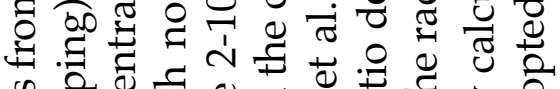

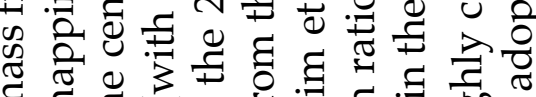

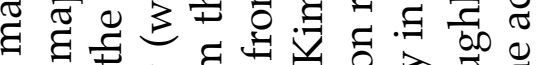

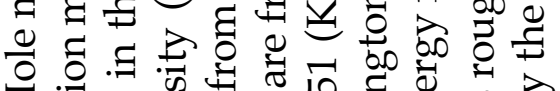
I

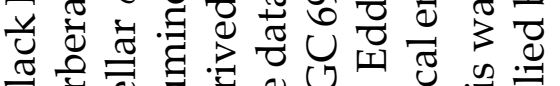

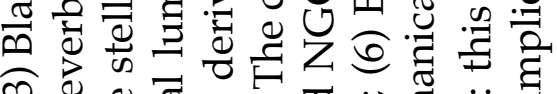

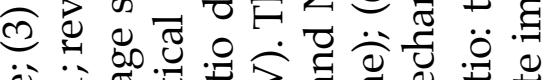

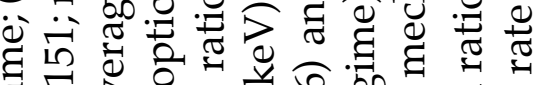
สี

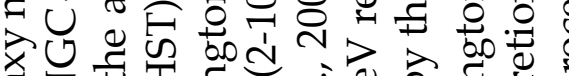

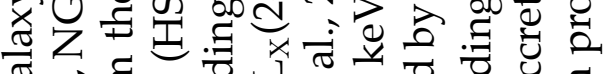
उٓ

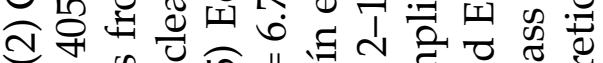

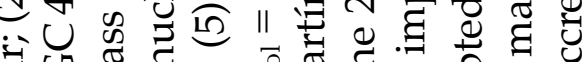

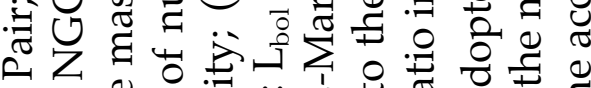
서유.

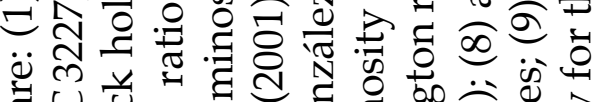

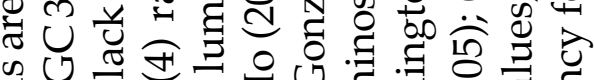

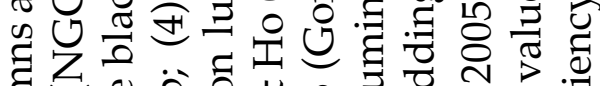

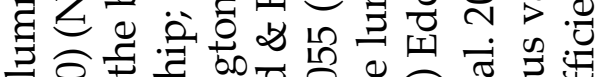

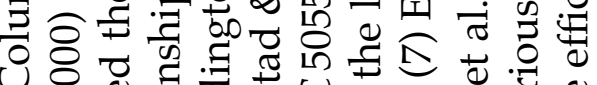
ن

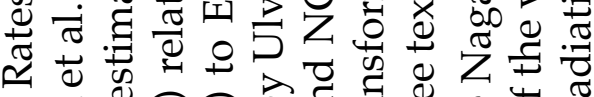

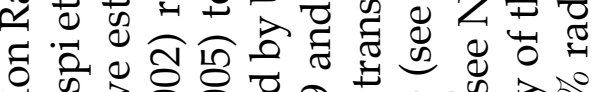
.尹

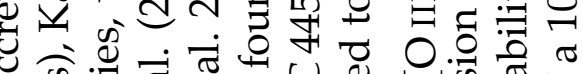

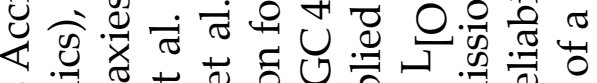

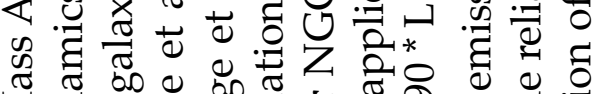
胥 ర

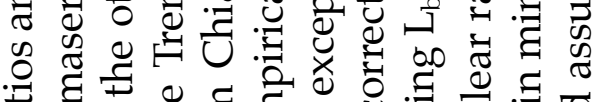

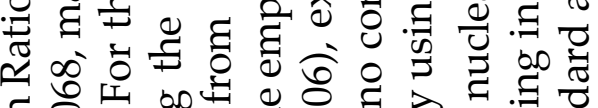
당 $00+$ \& 8 至

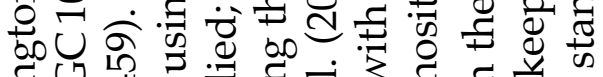

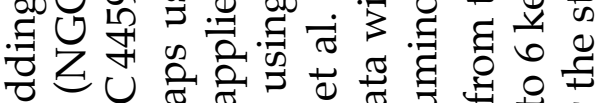

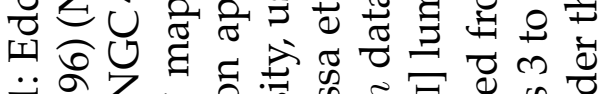

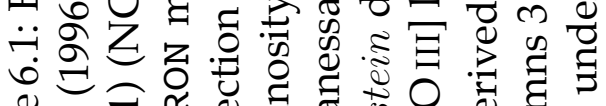

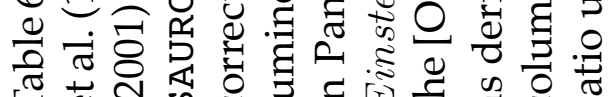




\subsection{Accretion rates and kinematic misalignments}

In this section we highlight the relationship between kinematic disturbances in the circumnuclear regions of the galaxy and the accretion rate of the nuclear black hole. Throughout this study we used the nuclear type classification of Ho et al. (1997). Figure 6.1 presents our galaxies in the diagnostic diagram $\log ([\mathrm{O} \mathrm{III}] / \mathrm{H} \beta)$ vs $\log ([\mathrm{N} \mathrm{II}] / \mathrm{H} \alpha)$, as well as the boundaries between the different excitation classes adopted by Ho et al. (1997). The separation between Seyfert and LINERS objects is not as sharp as depicted by this diagnostic diagram. We can notice that NGC 5055 is close to be a Seyfert while NGC 3627 and NGC 4579 are Seyferts and close to the LINERS and Transition objects. The activity classification of the sample nuclei therefore does not necessarily accurately represent the nuclear black hole accretion rate.

We have then estimated the Eddington rates and the corresponding mass accretion rates of the individual nuclei from four relatively independent methods, assuming even our non-active galaxies contain an accretion disk around a $\mathrm{SMBH}$, at very low activity level, and then that the central luminosity considered in the calculus of mass accretion come from this accretion disk. The results are summarised in Table 6.1. The black hole masses in this Table (column 3) are from direct dynamic measurements (e.g. NGC 1068), from reverberation mapping results (e.g. NGC 4051, NGC 4151), or estimated from the central stellar velocity dispersion in our SAURON maps via the well-studied M- $\sigma$ relationship (e.g. Tremaine et al., 2002, see Chap. 2). We have used four observational estimates for the Eddington ratio of the nuclei. The first is the optical continuum luminosity of the galaxy nucleus from HST images (column 4 of Table 6.1), interpreted as optical synchrotron radiation from the base of the jet (Chiaberge et al., 2005). No bolometric correction is applied so the result should be considered a lower limit to the Eddington ratio. Of course, contamination of the nuclear optical continuum from star formation could cause the AGN-related luminosity value to be lower. We have also used the hard (2-10 $\mathrm{keV}$ ) X-ray luminosity, the nuclear [O III] luminosity (from Panessa et al. 2006 and Ho et al. 1997) and the nuclear radio power to estimate the bolometric luminosity of the nucleus (columns 5 to 7 of Table 6.1). In the case of the [O III] luminosity, we tested the estimator used for active galaxies in the SDSS - $\mathrm{L}_{\mathrm{bol}}$ $=3500 \times \mathrm{L}_{\text {[O III] }}$ (Heckman et al., 2004). This formula resulted in too high values of $\mathrm{L}_{\mathrm{bol}}$ for our sample. The factor 3500 derived by Heckman et al. (2004) was based on the empirically derived ratios $\mathrm{L}_{5000} / \mathrm{L}_{[\mathrm{O} \text { III }]}=320$, where $\mathrm{L}_{5000}$ is the monochromatic continuum luminosity $\lambda \mathrm{P}_{\lambda}$ at $5000 \AA$ and $\mathrm{L}_{\mathrm{bol}} / \mathrm{L}_{5000}=10.9$. Heckman et al. (2004) based and tested this relationship mainly on AGN with Log [O III] luminosities of 6.5 and above. Our sample has much lower Log [O III] luminosities, ranging from 3.8 to 8.3 , with median value 5.1. We find that a factor of 90 , instead of 3500 , gives a more reasonable value for the bolometric luminosity within our sample. This lower factor could reflect a higher [O III] emission relative to $\mathrm{L}_{5000}$ from our lower luminosity, gas rich systems as compared to the SDSS Seyferts. In the case of nuclear radio luminosities, 
we used the sub-arcsecond AGN-related radio flux to calculate the implied jet mechanical power (see Nagar et al. 2005 for details). Nagar et al. (2005) have demonstrated that this method to calculate the bolometric luminosity works well in low luminosity AGN. For each nucleus, we then adopted a value of $l_{\text {Edd }}=\mathrm{L}_{\mathrm{bol}} / \mathrm{L}_{\mathrm{Edd}}$ (column 8 of Table 6.1) from the previous four values, keeping in mind their relative uncertainties and the paradigm that low luminosity AGN in the low (high) state are more likely to produce radio (X-ray) emission. This adopted value of the Eddington ratio was used to calculate the estimated mass accretion rate (column 9). The inactive galaxies NGC 3351, NGC 5248, and NGC 5806, all three with HII-type nuclei, have not been detected in the hard Xray nor radio. Their Eddington ratios are likely lower than the value adopted in Table 6.1, since a significant part of their nuclear [O III] luminosity could come from HII regions. Note that while the overall Seyfert and inactive samples show expected different Eddington ratios, there is some overlap; two of the Seyferts, NGC 3627 and NGC 6951, have Eddington ratios similar to the inactive nuclei (see Fig. 6.2). From the diagnostic diagram, NGC 3627 is close to be classified as LINER and NGC 6951 has been previously classified as LINER, or composite object between high excitation LINER and Seyfert 2 (Marquez \& Moles, 1993; Pérez-Ramírez et al., 2000).

The relationship between mass accretion rate and the kinematic of the gas and stars is illustrated in Fig. 6.2. The result is rather striking: black holes with low accretion rates reside in galaxies in which the stellar and gas kinematic axes are closely aligned and in which the gas kinematic axis is least disturbed, while black holes with accretion rates higher than $10^{-4.5} \mathrm{M}_{\odot} / \mathrm{yr}$ reside in galaxies with either a large misalignment between gas and stellar axes, or a large twist in their gas kinematic axes. Two Seyfert galaxies (NGC 4151 and 4579) have high accretion rate, large kinematic misalignment and present relatively undisturbed gaseous kinematics, while NGC 1068 and 4051 present no significant misalignment but large perturbations in the gaseous kinematics. Except for the two Seyfert galaxies with low accretion rate (NGC3627 and 6951), these plots show the same trend discussed above, Seyfert galaxies display higher kinematics misalignment and more perturbed gaseous kinematics than the no-active galaxies.

This result does not show, however, any direct correlation between the accretion rate and observed kinematic misalignment. Recent numerical studies (King \& Pringle, 2007, 2006; Hopkins \& Hernquist, 2006) suggest that lowluminosity AGN can be efficiently triggered and fuelled via series of stochastic accretion events on small-scale (from $\sim 100 \mathrm{pc}$ to sub-parsec regions). Then, dynamics of the host galaxy seem to play an important role in the accretion of gas from the outskirt of the galactic disk to the inner $\sim 100 \mathrm{pc}$, and physics of the gas, viscous processes become predominant in the accretion disk, and funnel the material closer to the central black holes, sustaining the nuclear activity. Observed accretion rates, and in particular time-scale and high variability involved in accretion onto $\mathrm{SMBH}$, are constrained by the physics inside the accretion disk itself. 


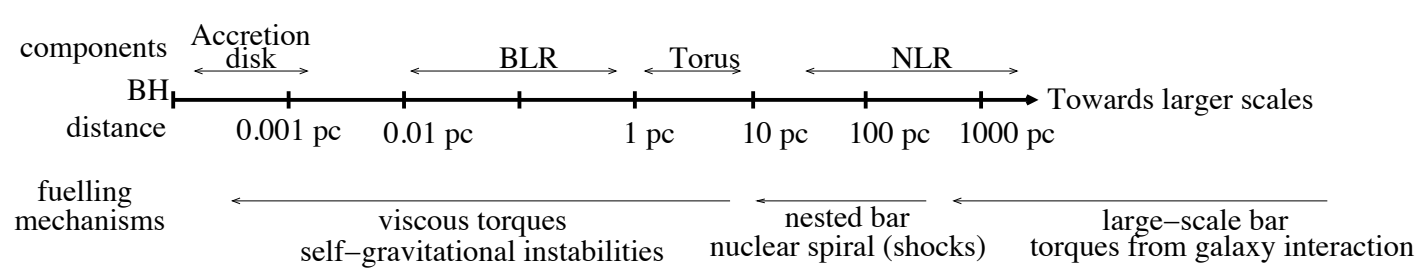

Figure 6.3: Several important fuelling mechanisms are represented on the galactic spatial scales where they are predominant, for a SMBH of mass $10^{7}$ $\mathrm{M}_{\odot}$. The different AGN components (accretion disk, BLR, dusty torus and NLR) are also shown for comparison. The distances from the central black hole are displayed in log.

Fig. 6.3 provides an idea of several possible mechanisms which can remove angular momentum of the gas and are predominant relative to other processes at different spatial scales in the galactic disks. Dynamical structures in the host galaxy (bars, spiral arms, gravitational torques due to galaxy interactions) seem to play an important role in funnelling gas within $10 \mathrm{pc}$. Then, viscous processes, hydrodynamic instabilities take over and transport the gas closer to the SMBH.

\subsection{Perspectives}

Why a fraction of nearby galaxies host an active nucleus remains an unsolved question. As presented in Chapter 2, several mechanisms may be involved in the fuelling of the central regions of galaxies, and subsequently activating and sustaining nuclear activity. What mechanism is dominant in the case of nearby low-luminosity AGN and what is the influence of the galaxy host on the fuelling are still elusive.

In this thesis, I presented an ambitious and comprehensive multiwavelength study of the two-dimensional kinematics and morphology of a sample of Seyfert and non-active galaxies. The active and non-active galaxies have well-matched optical host properties (total luminosity, morphological type, disk inclination) and therefore form pairs on which comparative analyses are done. One goal of this study is to investigate the kinematics of Seyfert and non-active galaxies, in order to reveal any difference between the two samples, which could be signatures of the underlying fuelling mechanisms. The first results of the study presented here are the following:

- In the circumnuclear regions, the stellar velocity maps present regular rotation patterns for both Seyfert and non-active galaxies. The stellar kinematic major-axis is a robust determination of the LON of the galaxy in our sample, except for two Seyfert 2 galaxies: NGC 1068 and 5194. The outer H I kinematic PA is also similar to the stellar kinematic PA in the circumnuclear regions. 
- The ionised gas co-rotates generally with the stars in the circumnuclear regions. Only two Seyfert galaxies NGC 2655 and 4579 present unambiguous large kinematic misalignments between stars and gas.

- The gaseous kinematic is more perturbed than the stellar one for both Seyfert and non-active galaxies. However the Seyfert galaxies present higher variations of stellar and gaseous kinematic PA than non-active galaxies. This trend may indicate a link between the galactic host dynamics in the circumnuclear regions and the nuclear activity.

The next step of this study is to investigate the galactic kinematics with more refinement, in order to identify and eliminate any possible triggering and fuelling mechanisms of the nuclear activity in the Seyfert galaxies.

\subsubsection{Harmonic analysis of velocity fields}

As presented in Chapter 5, harmonic analysis of large-scale neutral gas disk, circumnuclear stellar and ionised gas velocity fields can be performed. Once the rotational velocity has been estimated with PyRing (see Sec. 5.3), $V_{\text {sys }}$, $\left(x_{c}, y_{c}\right), i$ and the PA are fixed to their best-fitting values and the $c_{n}$ and $s_{n}$ coefficients are adjusted iteratively for each ring, by fitting the expression of the line-of-sight velocity of Eq. 5.4 up to the third harmonic order.

Radial variations of the harmonic coefficients can be interpreted as signatures of the dynamical structures responsible for non-circular motions. This analysis has been detailed by Schoenmakers et al. (1997). The simplest case of non-circular motions is an axisymmetric radial flow. Then all the harmonic coefficients are zero, except $c_{0}=V_{s y s}, c_{1}=V_{\Phi} \sin i$ and $s_{1}=V_{R} \sin i$, following the notation of Sec. 5.2. Considering now a general perturbation of an axisymmetric potential, of harmonic $m$, Schoenmakers et al. (1997) showed that signatures of such dynamical perturbations occur in the $(m-1)$ th and $(m-1)$ th harmonic coefficients of the velocity field. These coefficients can then be expressed as a function of the parameters of the dynamical perturbation. For example, in the case of a $m=2$ instability, such as a bar or spiral, the $c_{1}, s_{1}, c_{3}$ and $s_{3}$ can be expressed with the parameters of the perturbations, e.g. pattern speed $\Omega_{p}$, PA of the bar or pitch angle of the spiral. Hence, investigating the variations of the harmonic coefficients of the velocity fields allows to constrain the underlying potential of the galactic disk.

Wong et al. (2004) discussed the influence on the Fourier harmonic coefficients of several simple cases of dynamical structures: a pure radial streaming, motions in a $m=2$ instability such as a barred potential or a spiral wave. Finally, Wong et al. (2004) showed that the first and third even coefficients $\left(s_{1}\right.$ and $s_{3}$ ) variations are a good indicator for e.g. a warp, streaming in a barred potential and pure radial inflows: 
- A bar is the predominant structure if the $s_{1}$ and $s_{3}$ coefficients are anticorrelated, with $d s_{1} / d s_{3}<0$

- A pure radial inflow is occurring if the $s_{1}$ coefficients is significant in some regions and $d s_{3} \approx 0$

- A warp is the predominant structure if the $s_{1}$ and $s_{3}$ coefficients are correlated, with $d s_{1} / d s_{3}>0$

The last point mentions the trend in the $s_{1}-s_{3}$ relation in the case of a warp in the galactic disk. Although a warp is not directly related to gaseous streaming, the inclination and PA of the galactic disk may change. It implies that a warp will affect the harmonic coefficients of the velocity fields as if wrong values of $i$ and PA were used. Schoenmakers et al. (1997) investigated in details how errors in the geometrical parameters (dynamical centre, $i$, PA) affect the harmonic coefficients. Effects of a warp should be negligible in the case of our optical data since only the circumnuclear regions are mapped. It is not the case for the radio data, since warps are common in the H I outer disks. Such effect must be kept in mind when the interpretation of the non-circular velocity components of the $\mathrm{H}$ I data is performed. In this case, we expect the $s_{1}$ and $s_{3}$ to lie along a line refer as the warped line of slope : $\frac{d s_{1}}{d s_{3}}=\frac{1-q^{2}}{3 q^{3}+1}$, where $q=\cos i$ (Wong et al., 2004).

In our study, we assume the $s_{1}$ coefficient to be interpreted as radial inflow or outflow, depending on the sign of $s_{1}$ and on the sense of rotation of the galaxy. If the galaxy rotates counter-clockwise, positive values of $s_{1}$ correspond to radial outflow, otherwise, they correspond to radial inflow. In order to determine the sense of rotation of the galaxy, we consider that spirals arms are always trailing and/or investigate by eye the dust absorption to identify the nearest side.

Figs. 6.4 to 6.5 present the results of the tilted ring fit with harmonic coefficients up to the third order for three of our galaxies: NGC 2967, 4041 and 5055. The observed, model and residual velocity fields are displayed, as well as the radial variations of the $c_{1}, s_{1}$ and $s_{3}$ coefficients and the $s_{3}$ vs $s_{1}$ diagrams. For each plot the relative values $s_{1} / c_{1}$ and $s_{3} / c_{1}$ are computed. The warped line (Wong et al., 2004) is also displayed in the $s_{1}-s_{3}$ plane.

For NGC 2967, 5383 and 5055, the behaviour of the harmonic coefficients are good examples of the three models pointed out by Wong et al. (2004):

- NGC 2967 is a clear candidate for radial flows, with strong $s_{1}$ coefficient and $s_{3} \approx 0$ up to $200^{\prime \prime}$.

- NGC5055 H I kinematics presents $s_{3}$ and $s_{1}$ variations characteristic of motions in a warped disk (Fig. 6.4). Indeed, this galaxy presents an extended warped H I disk (Battaglia et al., 2006). In contrast, $s_{3}$ and $s_{1}$ are anti-correlated for the innermost regions, as shown in the figures corresponding to the ionised gas kinematics. This result suggests streaming 
motions in a barred or oval potential (Wong et al., 2004) and is in very good agreement with those of Wong et al. (2004) who analysed the H I kinematics in the large-scale disk and the $\mathrm{CO}$ kinematics in the circumnuclear regions.

- Finally, the slope of the $s_{1}-s_{3}$ relation is negative in the case of NGC 2967, indicating streaming in a bar potential. Indeed this galaxy hosts a largescale stellar bar (see Fig. B.1).

\subsubsection{Other directions for further works.}

- Connecting the large-scale disk and circumnuclear regions.

The simple criteria of Wong et al. (2004) discussed in the previous section are good indicators for signatures of radial flows in the galactic disk and allow to disentangle errors in the viewing angles values ( $i$ and PA) and warps from real non-circular components. They cannot however fully constrain all the dynamical structures. One needs to model the underlying potential and constrain the parameters of this model via fits of the harmonic coefficients to predict what are the predominant dynamical mechanisms at work and to quantify them, as done by e.g. Fathi et al. (2005). The results of such quantitative analysis on the circumnuclear regions, e.g. mapped with SAURON, are however difficult to interpret without information on the larger spatial scales. Similarly, constraining the dynamics of the large-scale disk, with no data in the innermost regions cannot probe the mechanisms which transport the gas close to the SMBH.

Therefore, this quantitative Fourier analysis of our multi-scale velocity fields will allow to constrain the galactic gravitational potential from the outskirts of the galaxy down to the circumnuclear regions, then identify or eliminate potential AGN fuelling mechanisms.

\section{- Probing the angular momentum.}

Another way to analyse the galactic stellar velocity fields has been developed recently by Emsellem et al. (2007) and Cappellari et al. (2007). Emsellem et al. (2007) presented a new kinematic parameter $\lambda_{R}=<R|V|>/ \sqrt{V^{2}+\sigma^{2}}$ which quantifies the projected stellar angular momentum per unit mass, $V$ and $\sigma$ being the stellar velocity and velocity dispersion. In particular the radial variations of $\lambda_{R}$ may characterise the regions of low and high angular momentum, and Emsellem et al. (2007) define two classes, namely low of fast rotators, depending on the values of $\lambda_{R}$ within the effective radius of the galaxy (for early-type galaxies observed with SAURON). Cappellari et al. (2007) showed that this classification can be related to the merger tree followed by the galaxy. For galaxies in our sample, probing the central baryonic angular momentum could lead to interesting clues regarding their dynamical histories. 

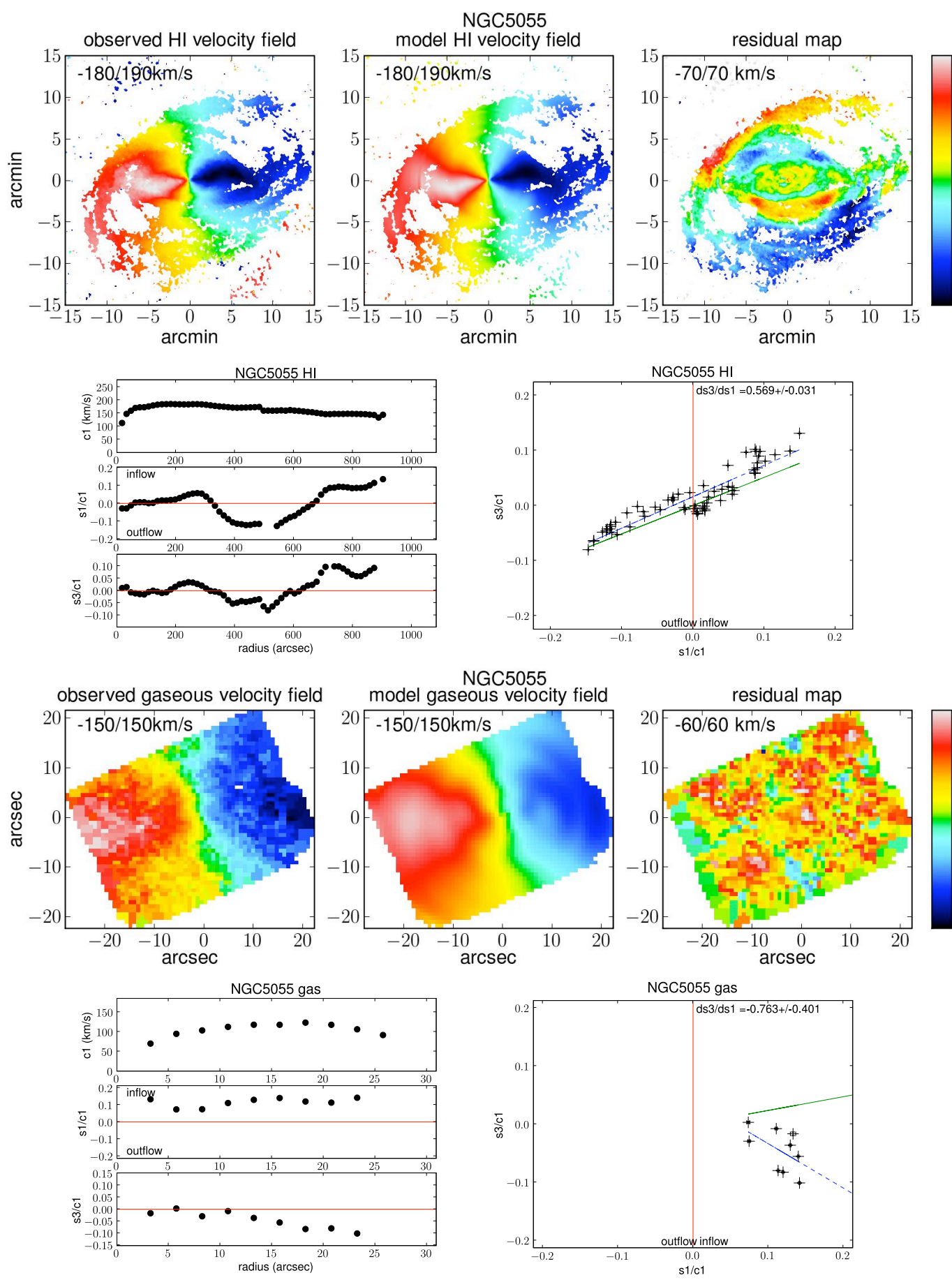

Figure 6.4: Harmonic analysis of NGC $5055 \mathrm{HI}$ (bottom plots) and ionised gas (top plots) velocity fields. For each component, the top panel presents the observed velocity field, modeled velocity field and the residual map. Then, in the bottom left panel the radial variations of the $c_{1}, s_{1}$ and $s_{3}$ coefficients are shown. Finally the $s_{3}$ coefficient is plotted as a function of $s_{1}$ in the bottom right panel. The $s_{1}$ and $s_{3}$ coefficients are normalised to the $c_{1}$ coefficient, assuming it represents the projected rotational velocity. The green and blue lines correspond to the warp line (expected relation between $s_{3}$ and $s_{1}$ for a pure warp model) and a linear least-squares fit to the data points, respectively. The slope of the least-squares fit is given in the top right corner of the figure. 

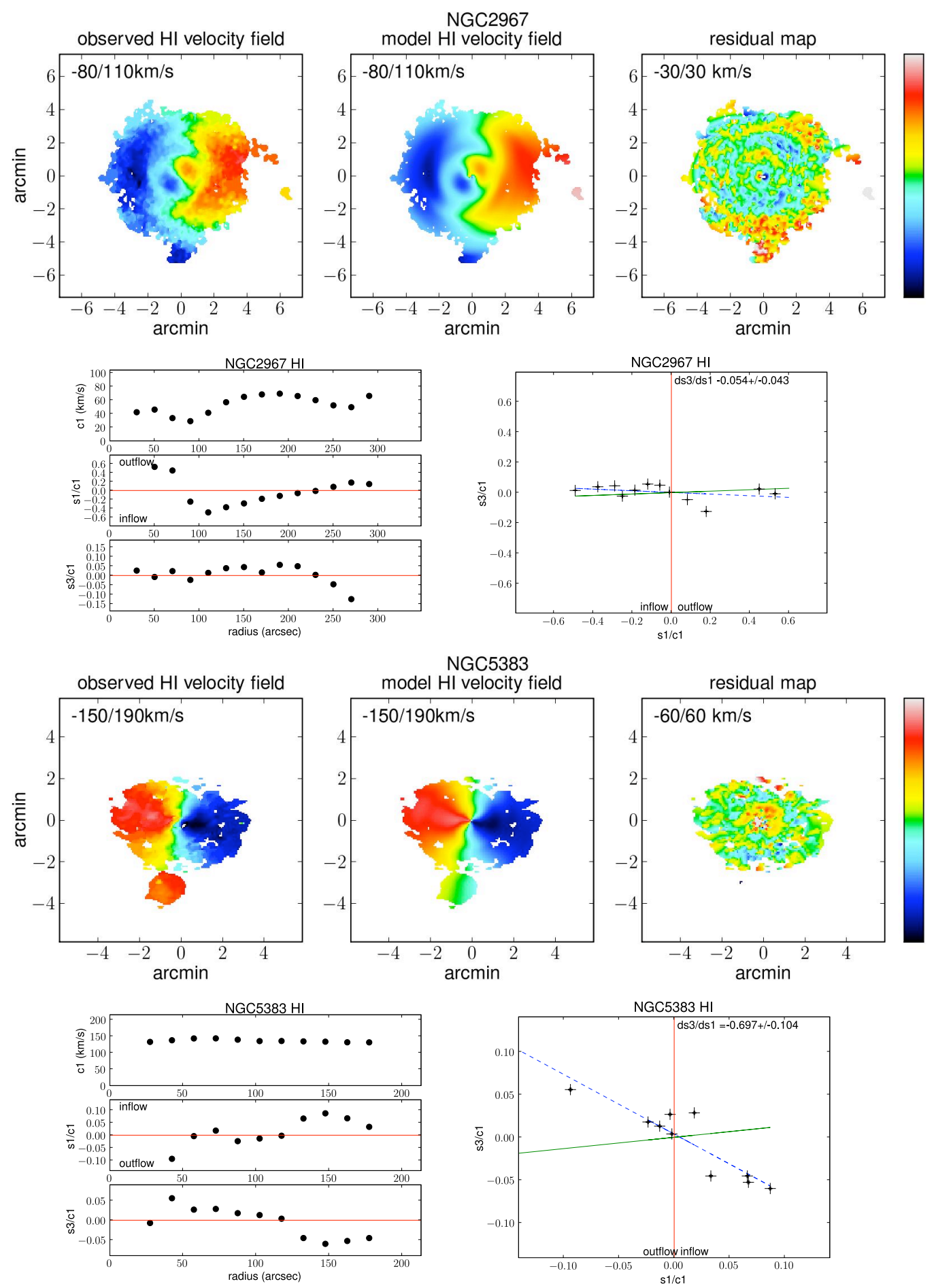

Figure 6.5: Harmonic analysis of the non active galaxies NGC 2967 (top plots) and NGC5383 (bottom plots) H I velocity fields. For each component, the top panel presents the observed velocity field, modeled velocity field and the residual map. Then, in the bottom left panel the radial variations of the $c_{1}, s_{1}$ and $s_{3}$ coefficients are shown. Finally the $s_{3}$ coefficient is plotted as a function of $s_{1}$ in the bottom right panel. The $s_{1}$ and $s_{3}$ coefficients are normalised to the $c_{1}$ coefficient, assuming it represents the projected rotational velocity. The green and blue lines correspond to the warp line (expected relation between $s_{3}$ and $s_{1}$ for a pure warp model) and a linear least-squares fit to the data points, respectively. The slope of the least-squares fit is given in the top right corner of the figure. 
- Physics of the gas

Finally, the physics of the gas in the innermost regions must play an important role in the fuelling of the AGN. Therefore, investigating the physical condition of the gas and the hydrodynamical processes at work in these regions would probe the fuelling mechanisms. Our neutral and ionised gas data, combined with molecular gas data from the NUGA survey would provide for the first time unique information of the gaseous component at different spatial scales and in different phases. 


\section{Appendix A}

\section{The master sample}

Table A.1 lists the properties of the galaxies in our master sample. The last two columns noticed whether radio (Column 13) or optical (Column 14) data are available. " 1 " or " 0 " mean that corresponding data are available or unavailable, respectively. 


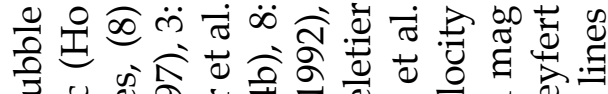
至

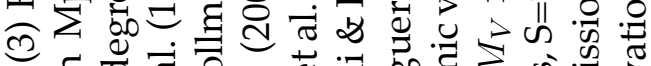

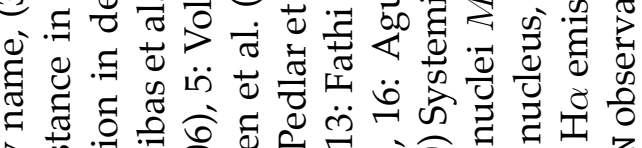

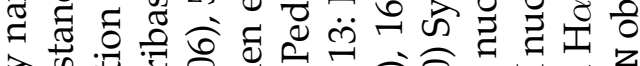

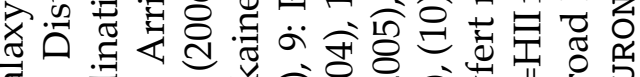

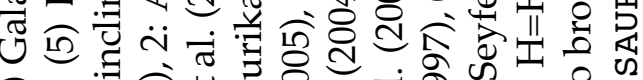

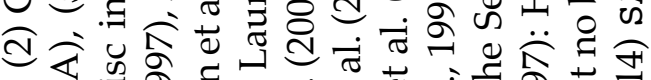
む)

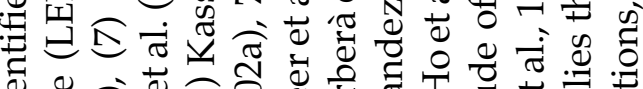
ฮัँ

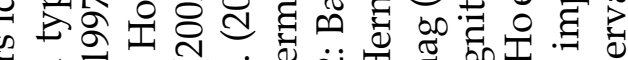

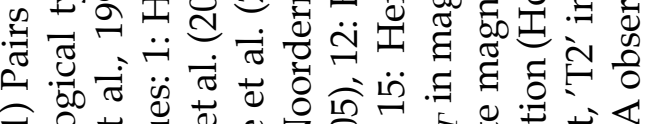

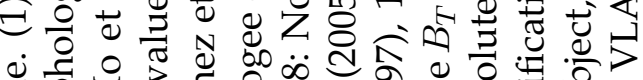

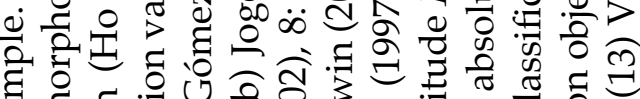
苟

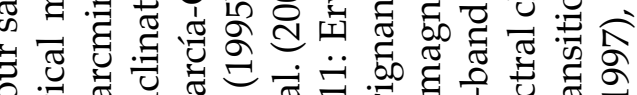

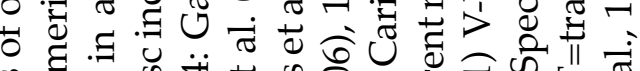
थ

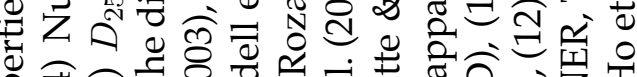

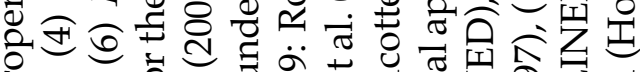

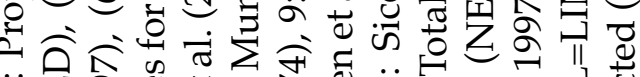

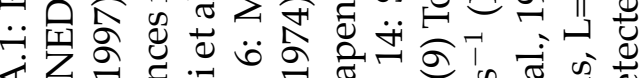

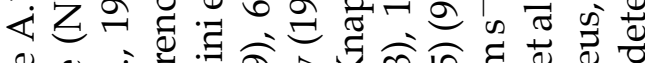

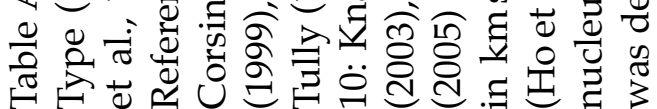

\begin{tabular}{|c|c|c|c|c|c|c|}
\hline 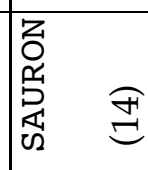 & $-r-$ & 00 & $\begin{array}{ll}0 & 0\end{array}$ & $\begin{array}{ll}1 & 0\end{array}$ & $\begin{array}{lll}-1 & 0 & 0\end{array}$ & -1 \\
\hline 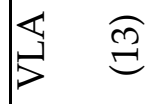 & $-r-$ & 00 & $\neg-$ & $-1-1$ & $-10-$ & - \\
\hline 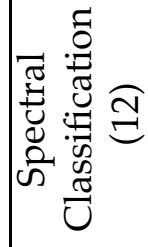 & कै & $\frac{n}{\infty}$ & W $I$ & $\left|\begin{array}{ll}\infty & \mathcal{\sim} \\
\dot{\omega} & \ddots\end{array}\right|$ & 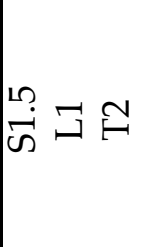 & 产 \\
\hline 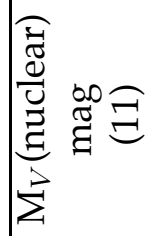 & & 足 & 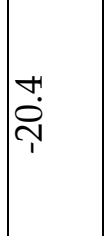 & & $\frac{\infty}{\infty}$ & مे \\
\hline$P^{\substack{n \\
\vec{m} \\
p^{2}}}$ & 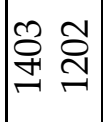 & 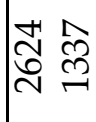 & 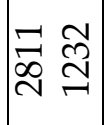 & $\begin{array}{ll}\stackrel{ }{\infty} & \infty \\
\stackrel{D}{二} & \infty \\
+ & \infty\end{array} \mid$ & 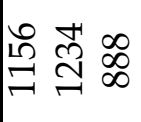 & হু \\
\hline ఐ & 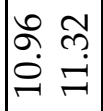 & 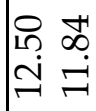 & 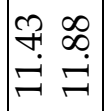 & 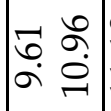 & 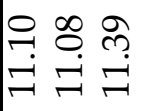 & 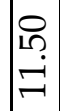 \\
\hline 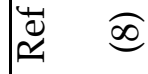 & $\neg-$ & $N m$ & $-r-7$ & $\begin{array}{lll}+1 \\
0\end{array}$ & $6 \wedge \infty$ & $a$ \\
\hline 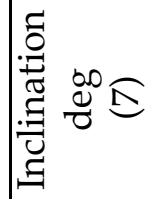 & लि F & 와 유 & $\hat{\lambda} \vec{\sim}$ & $\begin{array}{ll}N & \infty \\
\infty & \infty\end{array} \mid$ & 舫 & fo \\
\hline คి & 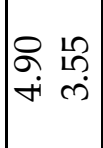 & 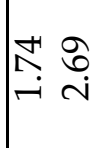 & $\begin{array}{ll}\infty & 0 \\
\infty & 0 \\
\dot{\infty} & \dot{j}\end{array} \mid$ & 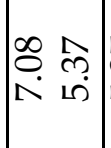 & 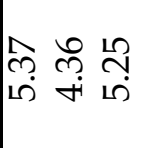 & $\begin{array}{l}0 \\
0 \\
0\end{array}$ \\
\hline 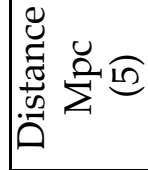 & $\begin{array}{ll}+ & \infty \\
\stackrel{+}{+} & 0 \\
\end{array}$ & $\begin{array}{ll}\infty & m \\
\tilde{m} & \tilde{N}\end{array}$ & $\begin{array}{ll}\hat{\sigma} & \widehat{a} \\
\hat{q} & \mathrm{~N}\end{array}$ & 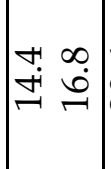 & 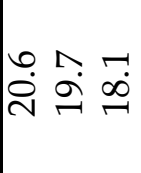 & m. \\
\hline$\mapsto \quad \nRightarrow$ & $\mid \begin{array}{ll}0 & 0 \\
0 & \stackrel{T}{1}\end{array}$ & 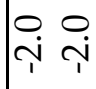 & $\stackrel{\circ}{\stackrel{\circ}{+}} \stackrel{0}{+}$ & $\left|\begin{array}{cc}0 & 0 \\
\dot{\infty} & \dot{m}\end{array}\right|$ & ن. & ì \\
\hline 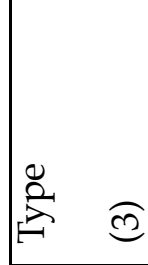 & 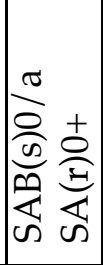 & 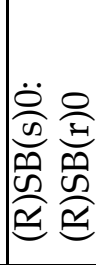 & 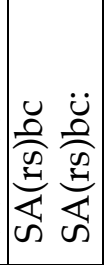 & 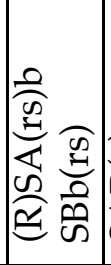 & 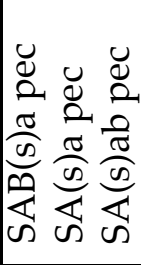 & 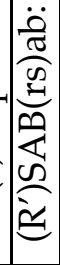 \\
\hline $\begin{array}{l}\mathscr{\Xi} \\
\text { z }\end{array}$ & 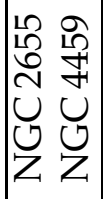 & 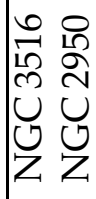 & 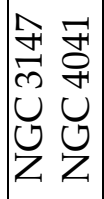 & 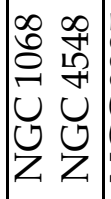 & 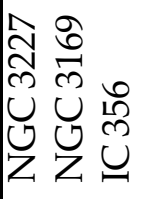 & $\begin{array}{l}\overrightarrow{10} \\
\overrightarrow{7} \\
0 \\
0 \\
Z \\
Z\end{array}$ \\
\hline$\stackrel{\Xi}{\tilde{2}} \circlearrowright$ & & 0 & $m$ & $\forall$ & Lم & 6 \\
\hline
\end{tabular}




\begin{tabular}{|c|c|c|c|c|c|c|c|c|c|c|c|c|c|c|}
\hline 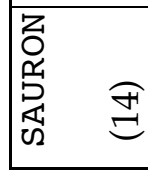 & -1 & 100 & 00 & 100 & $\neg-$ & 100 & 00 & $\mid \begin{array}{ll}0 & 0\end{array}$ & $\begin{array}{ll}0 & 0\end{array}$ & $\mid \begin{array}{ll}0 & 0\end{array}$ & $\mid \begin{array}{ll}0 & 0\end{array}$ & $\mid \begin{array}{ll}0 & 0\end{array}$ & 100 & 0 \\
\hline 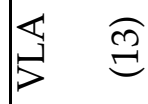 & -1 & $-r$ & $-1-$ & $-r$ & $-1-$ & $-1-$ & $-\neg-$ & $\mid \begin{array}{ll}0 & 0\end{array}$ & $\neg-$ & $-1-1$ & $-\neg-1$ & $\neg-1$ & $0-1$ & -1 \\
\hline 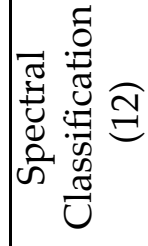 & 高 & $\bar{\omega}$ & $\frac{\sigma}{\omega}:$ & ஸे I & 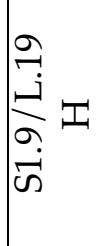 & एक & N $\Psi$ & $\mid \begin{array}{cc}\vec{i} & N \\
\omega & \end{array}$ & $\tilde{N}=$ & $\omega \cong$ & 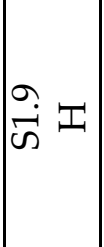 & $\omega \cong$ & एक & $\bar{s}$ \\
\hline 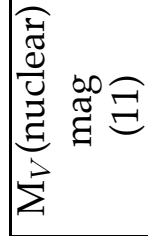 & & $\begin{array}{l}0 \\
9 \\
1 \\
1\end{array}$ & 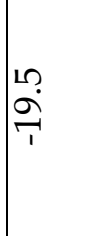 & $\frac{12}{19}$ & $\frac{+}{a}$ & $\frac{m}{2}$ & $\frac{m}{2}$ & $\begin{array}{l}\sigma \\
\infty \\
1 \\
1\end{array}$ & $\begin{array}{l}\infty \\
\infty \\
1 \\
1\end{array}$ & $\frac{1}{\infty}$ & $\begin{array}{l}1 \\
\infty \\
\frac{1}{1}\end{array}$ & $\begin{array}{l}0 \\
\infty \\
1 \\
1\end{array}$ & $\begin{array}{l}10 \\
\infty \\
1 \\
1\end{array}$ & $\begin{array}{l}+ \\
\infty \\
T \\
T\end{array}$ \\
\hline 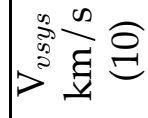 & $\frac{\infty}{\infty}$ & 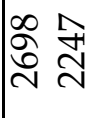 & 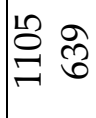 & $\begin{array}{ll}\infty & \infty \\
\infty & \infty \\
\hat{N} & \stackrel{n}{n} \\
& \end{array}$ & 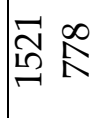 & $\begin{array}{cc}\hat{N} & \mathscr{8} \\
\infty & 0 \\
0\end{array}$ & 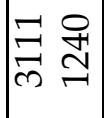 & 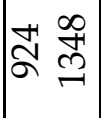 & 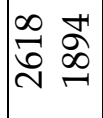 & $\left|\begin{array}{cc}0 & -1 \\
0 & \infty \\
\hdashline & 0 \\
-1 & -1\end{array}\right|$ & 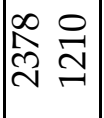 & $\mid$\begin{tabular}{cc}
$\infty$ & $\infty$ \\
0 & 0 \\
$\ddots$ & 0 \\
\hdashline & $=$
\end{tabular} & \begin{tabular}{ll}
$\begin{array}{l}1 \\
0\end{array}$ & 2 \\
0 & 0 \\
\hdashline & 0 \\
\hdashline
\end{tabular} & 造 \\
\hline 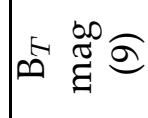 & 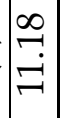 & 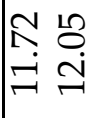 & 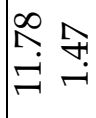 & 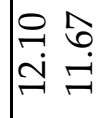 & 年 & 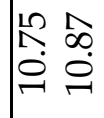 & 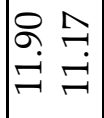 & 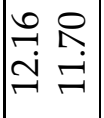 & 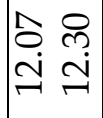 & 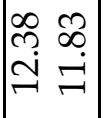 & 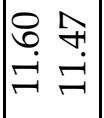 & 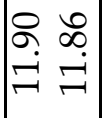 & 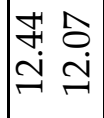 & İ \\
\hline$\stackrel{\mathbb{Q}}{\simeq}$ & $\infty$ & -1 & 으은 & $0 \infty$ & $m=$ & $\neg-$ & $\wedge \approx$ & $\wedge-1$ & $\wedge-$ & $\stackrel{m}{\rightarrow}$ & 윽요 이 & $\Lambda-1$ & $\stackrel{m}{-}-$ & -1 \\
\hline 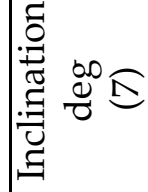 & $\infty$ & भ ल & શે & 능 우 & $\infty \begin{array}{l}\infty \\
\infty\end{array}$ & H̊ & $\mid \begin{array}{ll}\infty & \overline{10} \\
10 & \text {. }\end{array}$ & {$\left[\begin{array}{ll}n & \infty \\
10 & 10\end{array} \mid\right.$} & $\approx \mathbb{N}$ & $\widehat{\approx}$ ล & $F \Delta$ & $\begin{array}{lll}6 & 0\end{array}$ & 㳖 & \& \\
\hline คి & 命 & $\mid \begin{array}{ll}8 & 0 \\
\dot{m} & \dot{m}\end{array}$ & $\begin{array}{ll}3 & 8 \\
& \stackrel{0}{i}\end{array}$ & $\mid \begin{array}{ll}0 & a \\
\cdots & 0 \\
m & \stackrel{i}{1}\end{array}$ & \begin{tabular}{ll}
$\infty$ & 7 \\
$\infty$ & \multirow{7}{*}{}
\end{tabular} & $\begin{array}{ll}N & \bar{N} \\
0 & 0 \\
0 & \infty \\
- & \infty\end{array}$ & 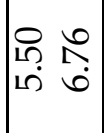 & 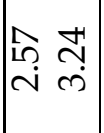 & 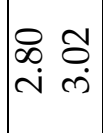 & $\left|\begin{array}{cc}\tilde{O} & \hat{\gamma} \\
\dot{\leftrightarrow} & \stackrel{+}{+}\end{array}\right|$ & $\left|\begin{array}{ll}\hat{O} & \& \\
+ & \stackrel{+}{+}\end{array}\right|$ & 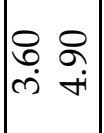 & 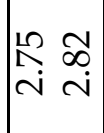 & 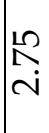 \\
\hline 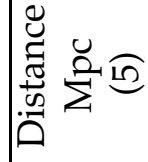 & ลู. & $\begin{array}{ll}0 & \infty \\
\dot{\infty} & \infty \\
\infty & \text { m }\end{array}$ & $\underset{0}{0} \stackrel{\infty}{=}$ & 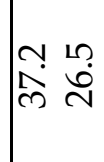 & $\stackrel{\infty}{\infty}$ & $\begin{array}{ll}1 & \infty \\
\infty & \infty \\
- & 0 \\
- & 0\end{array}$ & 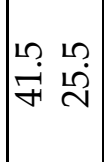 & 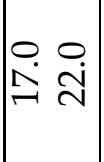 & $\begin{array}{ll}0 & 9 \\
\dot{m} & \vdots \\
m & \infty\end{array}$ & 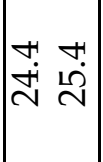 & 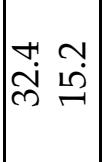 & $\left|\begin{array}{ll}\infty & 0 \\
\dot{\forall} & 0 \\
- & \underline{-}\end{array}\right|$ & $\begin{array}{cc}m & 0 \\
\ddot{N} & \dot{m}\end{array}$ & $\begin{array}{l}\infty \\
0 \\
0 \\
-1\end{array}$ \\
\hline$\mapsto \quad \nRightarrow$ & $\stackrel{0}{i}$ & $\begin{array}{cc}0 & 0 \\
\dot{m} & \dot{m}\end{array}$ & $\begin{array}{ll}\circ & \circ \\
\oplus & \oplus\end{array}$ & $\stackrel{\circ}{\rightarrow} \stackrel{0}{i}$ & $\stackrel{\circ}{\circ} \stackrel{\circ}{\oplus}$ & $\mid \begin{array}{ll}0 & 0 \\
10 & 10\end{array}$ & 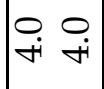 & 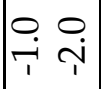 & $\left|\begin{array}{cc}0 & 0 \\
i n & 10 \\
10\end{array}\right|$ & 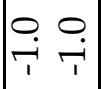 & $\left|\begin{array}{cc}0 & 0 \\
i n & 10 \\
10\end{array}\right|$ & $\left|\begin{array}{cc}0 & 0 \\
i & i\end{array}\right|$ & $\left.\mid \begin{array}{ll}0 & 0 \\
i & i \\
i\end{array}\right)$ & $\stackrel{\circ}{+}$ \\
\hline$\stackrel{0}{\infty}$ & 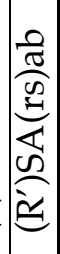 & 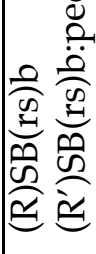 & 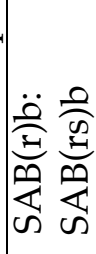 & 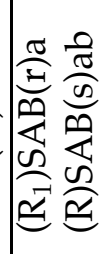 & 尊 & 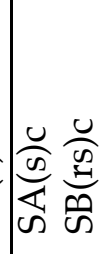 & 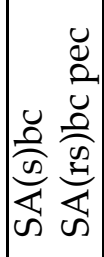 & 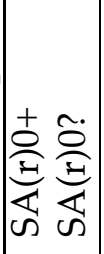 & 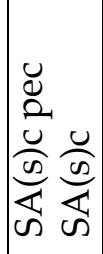 & 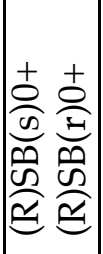 & 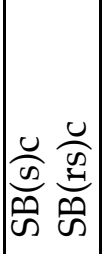 & 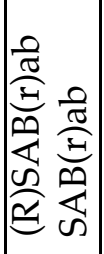 & 总 & 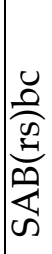 \\
\hline $\begin{array}{l}\mathscr{\Xi} \\
\tilde{\Xi} \\
Z\end{array}$ & 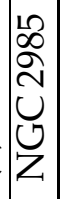 & \begin{tabular}{ll}
$\infty$ & 0 \\
0 & $\infty$ \\
1 & 0 \\
\hdashline & 10 \\
$ن$ & 0 \\
0 & 0 \\
$Z$ & $Z$
\end{tabular} & 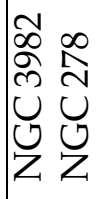 & 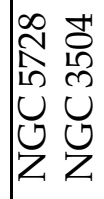 & 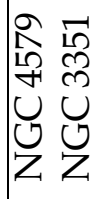 & $\begin{array}{ll}\infty & \infty \\
\tilde{D} & \sigma \\
10 & \infty \\
u & u \\
0 & 0 \\
z & z\end{array}$ & 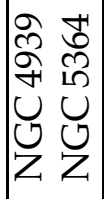 & 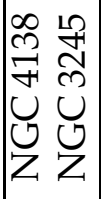 & 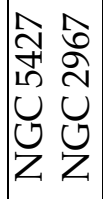 & 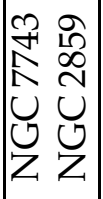 & 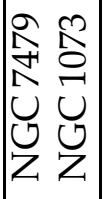 & 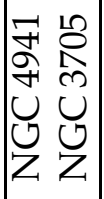 & 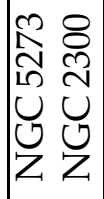 & 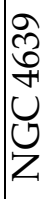 \\
\hline$\stackrel{\Xi}{\tilde{\Xi}} \quad \widehat{\Xi}$ & & $\Lambda$ & $\infty$ & $a$ & 잉 & 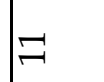 & $\approx$ & $\stackrel{m}{\longrightarrow}$ & $\nexists$ & 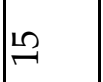 & $\stackrel{0}{-1}$ & 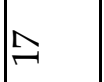 & $\infty$ & 2 \\
\hline
\end{tabular}




\begin{tabular}{|c|c|c|c|c|c|c|c|c|c|c|c|c|c|c|}
\hline 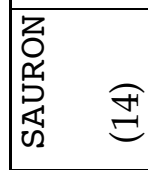 & 0 & $-1-$ & 00 & $\neg-1$ & 100 & $\mid \begin{array}{ll}0 & 0\end{array}$ & 100 & $\neg-$ & $-r-$ & $\mid \begin{array}{ll}0 & 0\end{array}$ & 100 & $\mid \begin{array}{ll}0 & 0\end{array}$ & 100 & 0 \\
\hline 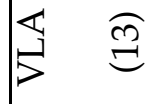 & -1 & $\neg-$ & $-r$ & $\neg-$ & $\neg-$ & $-r-1$ & $-r-$ & $\neg-$ & $-r-$ & $-\neg-1$ & 100 & $\mid \begin{array}{ll}0 & 0\end{array}$ & 100 & 0 \\
\hline 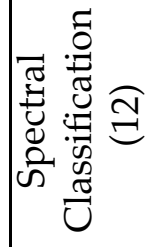 & 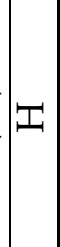 & 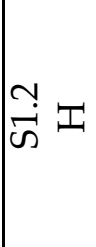 & $\approx \sim$ & क 1 & $\frac{\infty}{\dot{\omega}}: \Psi$ & $\frac{\sigma}{\dot{\omega}} \tilde{F}$ & 号 & $\frac{N}{N}=$ & $\approx N$ & $\left|\begin{array}{ll}\stackrel{n}{n} & N \\
\dot{\omega} & F\end{array}\right|$ & 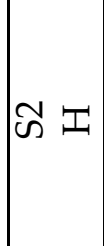 & 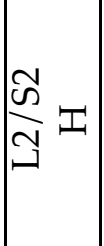 & 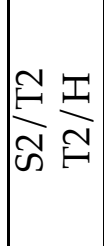 & N \\
\hline 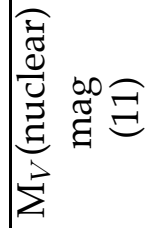 & & $\begin{array}{l}\text { N } \\
\infty \\
T \\
T\end{array}$ & $\frac{9}{1}$ & $\frac{9}{1}$ & 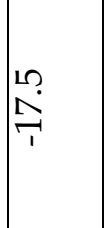 & 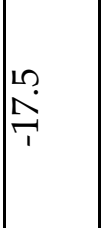 & $\frac{+}{1}$ & $\frac{N}{1}$ & $\begin{array}{l}0 \\
0 \\
1 \\
1\end{array}$ & $\stackrel{7}{+}$ & & & & \\
\hline 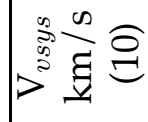 & $\begin{array}{l}8 \\
8 \\
\infty \\
-1\end{array}$ & 옷 & 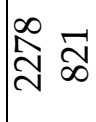 & 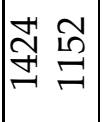 & 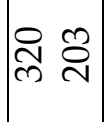 & 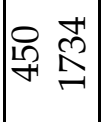 & $\left|\begin{array}{ll}3 & 0 \\
\infty & \infty \\
-\infty & m \\
-1\end{array}\right|$ & $\begin{array}{ll}\hat{N} & \infty \\
& \stackrel{0}{n} \\
\end{array}$ & \begin{tabular}{ll}
3 & 0 \\
\hdashline & 1 \\
\hdashline
\end{tabular} & 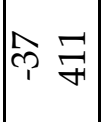 & $\begin{array}{ll}\infty & 0 \\
\text { in } & 0 \\
0\end{array}$ & 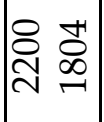 & 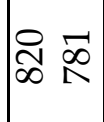 & $\infty$ \\
\hline صి & 곡 & 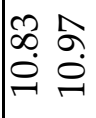 & 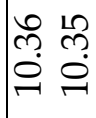 & 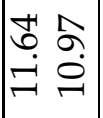 & 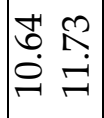 & 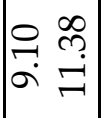 & 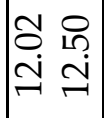 & 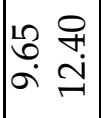 & $\begin{array}{ll}2 & \bar{n} \\
\infty & 0 \\
\infty & \sigma\end{array}$ & 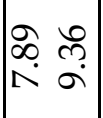 & 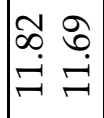 & 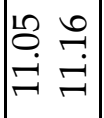 & 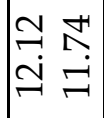 & 递 \\
\hline 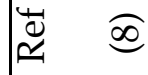 & -1 & $n$ & $\cong$ & $a r \mid \begin{array}{lll}a & 10\end{array}$ & - & $\because \triangle$ & 으 -1 & $m+1$ & $\infty \mathrm{\infty} \infty$ & $\simeq \cong$ & $\wedge \Lambda$ & $\stackrel{\Perp 0}{-}-1$ & $\infty-$ & $\approx$ \\
\hline 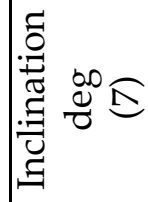 & ल & $\stackrel{9}{\rightarrow}$ 아 & 80 & m & ले & $\mid \begin{array}{lll} & 3\end{array}$ & $\vec{N}$ & 186 & గి ட & {$\left[\begin{array}{ll}10 & \text { นิ }\end{array}\right.$} & શે & $\infty \begin{array}{ll}\infty & 0\end{array}$ & $\overrightarrow{6}+$ & $F$ \\
\hline مَ & $\begin{array}{c}\sigma \\
\sigma \\
\dot{m}\end{array}$ & $\begin{array}{ll}\stackrel{1}{N} \\
\text { in } \\
\text { in }\end{array}$ & مै & 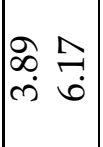 & $\left|\begin{array}{ll}\infty & \vdots \\
\ddot{\infty} & \vdots \\
\hdashline & \dot{0}\end{array}\right|$ & 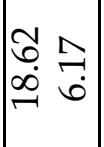 & $\left|\begin{array}{cc}8 & \stackrel{2}{0} \\
\dot{c} & \stackrel{i}{i}\end{array}\right|$ & 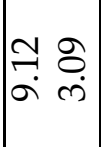 & 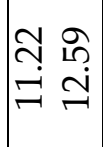 & مे & 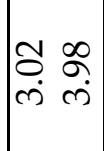 & 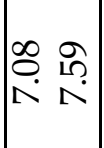 & 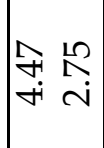 & م. \\
\hline 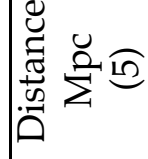 & ปู & 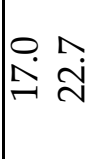 & $\stackrel{\infty}{\infty} \stackrel{n}{+}$ & تr & 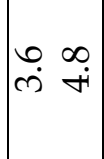 & $\left.\mid \begin{array}{ll}\infty & \stackrel{n}{0} \\
0 & \stackrel{\leftrightarrow}{\rightarrow}\end{array}\right]$ & 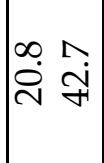 & $\left|\begin{array}{ll}0 & 0 \\
0 & 0 \\
0 & \stackrel{0}{N}\end{array}\right|$ & $\Lambda \stackrel{\Lambda}{\wedge}$ & 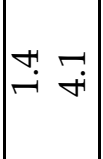 & $\because \bar{\sigma}$ & 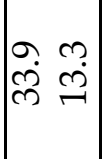 & $\mid \begin{array}{ll}\sim & \infty \\
0 & 0 \\
-1 & 0\end{array}$ & त! \\
\hline$\mapsto \quad \oiint$ & $\begin{array}{l}0 \\
+\end{array}$ & $\underset{+}{\circ} \stackrel{0}{+}$ & $\begin{array}{cc}\circ & \circ \\
\dot{m} & \dot{m}\end{array}$ & $\left|\begin{array}{cc}0 \\
\dot{+} & \stackrel{0}{+}\end{array}\right|$ & $\left|\begin{array}{ll}0 & 0 \\
\sigma & \ddots\end{array}\right|$ & $\left|\begin{array}{cc}0 & 0 \\
\dot{+} & \stackrel{+}{+}\end{array}\right|$ & $\left|\begin{array}{ll}0 & 0 \\
+ & +\end{array}\right|$ & $\left|\begin{array}{cc}0 & 0 \\
\dot{m} & \dot{m}\end{array}\right|$ & $\underset{+}{0} \stackrel{0}{+}$ & $\left|\begin{array}{ll}0 & 0 \\
i & i\end{array}\right|$ & $\left|\begin{array}{cc}0 & 0 \\
\dot{1 n} & 10\end{array}\right|$ & $\left|\begin{array}{cc}\stackrel{\circ}{+} & \stackrel{+}{+}\end{array}\right|$ & $\mid \begin{array}{cc}0 & 0 \\
i & i\end{array}$ & $\begin{array}{l}0 \\
10 \\
10\end{array}$ \\
\hline ᄅ & 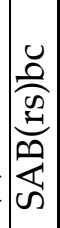 & 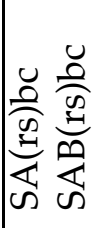 & 产产 & 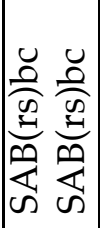 & 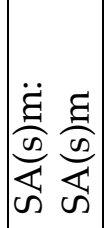 & 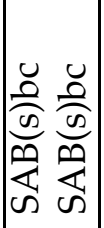 & 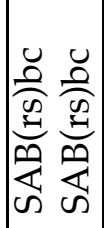 & 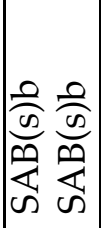 & 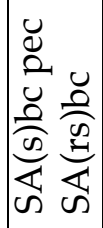 & 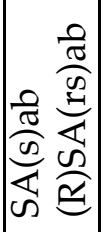 & 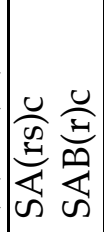 & 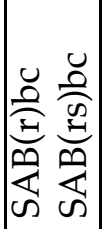 & 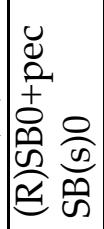 & 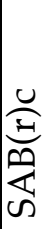 \\
\hline$\underset{\tilde{z}}{\stackrel{\Xi}{\Xi}}$ & $\begin{array}{l}\sigma \\
0 \\
m \\
u \\
0 \\
z\end{array}$ & 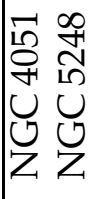 & 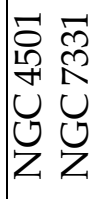 & 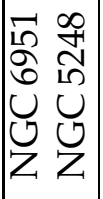 & 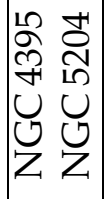 & 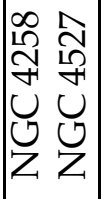 & $\mid \begin{array}{ll}\mathbb{1} & 0 \\
\infty & 0 \\
0 & 0 \\
u & 0 \\
0 & 0 \\
Z & 0 \\
Z & Z\end{array}$ & $\left|\begin{array}{ll}\hat{A} & 0 \\
0 & 0 \\
0 & 0 \\
ن & 0 \\
0 & 0 \\
\hdashline & 0 \\
z & Z\end{array}\right|$ & 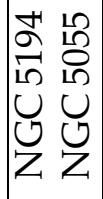 & $\left|\begin{array}{ll}\bar{c} & 0 \\
0 & 1 \\
0 & 0 \\
0 & + \\
u & u \\
0 & 0 \\
z & Z\end{array}\right|$ & 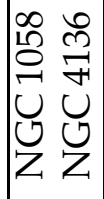 & 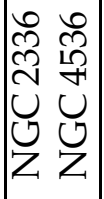 & 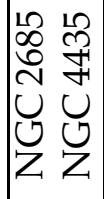 & 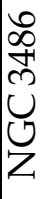 \\
\hline 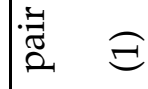 & & సิ & $\vec{N}$ & స̃ & సे & 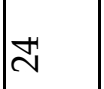 & $\stackrel{\sim}{\sim}$ & సి & సิ & సి & શิ & గ్లి & $\vec{m}$ & ले \\
\hline
\end{tabular}




\begin{tabular}{|c|c|c|c|c|c|c|c|c|}
\hline 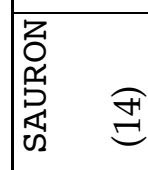 & 0 & 100 & 100 & 100 & 100 & 100 & 0 & 100 \\
\hline 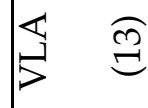 & 0 & 100 & 100 & 100 & 100 & 100 & 0 & 100 \\
\hline 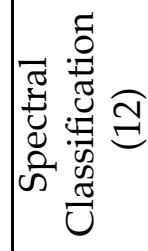 & 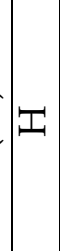 & $\frac{\tilde{N}}{\hat{N}}$ & $\frac{N}{\mathcal{W}^{\prime}} \Psi$ & N & ふ & ふै & ऊ) & $\overbrace{\hat{\beta}} \Psi^{\prime}$ \\
\hline 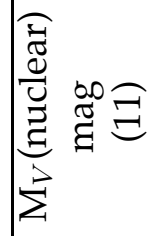 & & & & & & & & \\
\hline 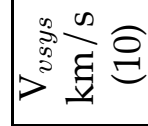 & $\infty$ & 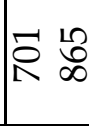 & 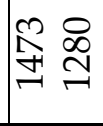 & స̃ & की & 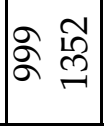 & (̊) & 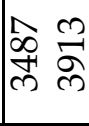 \\
\hline 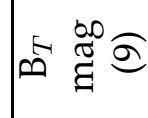 & 家 & 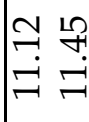 & 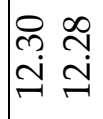 & 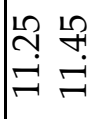 & 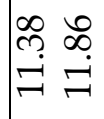 & 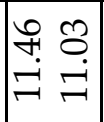 & \begin{tabular}{l}
$F$ \\
$\ddot{0}$ \\
\hdashline
\end{tabular} & 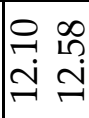 \\
\hline 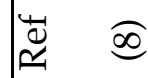 & $\Lambda$ & -1 & $\neg-$ & 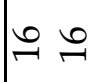 & $-r$ & $\Lambda-$ & $\infty$ & $-1-$ \\
\hline 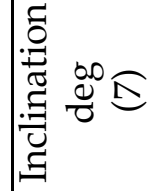 & in & مَ & டి ட & 암 L & $\overparen{\sim}$ & 낭 우 & $\vec{n}$ & 同 \\
\hline 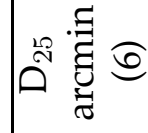 & $\frac{1}{6}$ & 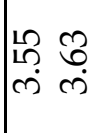 & 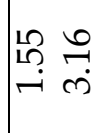 & 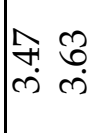 & $\begin{array}{ll}\infty & \mathbb{N} \\
\infty & \infty\end{array}$ & 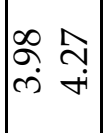 & $\left|\begin{array}{c}N \\
\hat{N} \\
0 \\
\hdashline\end{array}\right|$ & $\begin{array}{ll}\infty & 0 \\
\infty & + \\
ن & i\end{array}$ \\
\hline 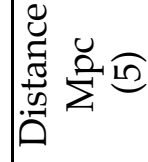 & $\begin{array}{l}0 \\
0 \\
-1 \\
-1\end{array}$ & : & 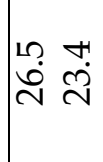 & $\stackrel{\sigma}{\infty} \underset{\infty}{\infty}$ & $\mid \begin{array}{ll}\infty & \infty \\
0 & 0 \\
-1 & 0\end{array}$ & $\mid \begin{array}{ll}\infty & 0 \\
0 & 0 \\
- & \therefore\end{array}$ & 孛 & 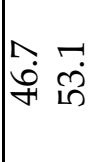 \\
\hline$F \quad$ ङ & 它 & $\mid \begin{array}{ll}0 & 0 \\
\rightarrow & \stackrel{1}{i}\end{array}$ & $\left|\begin{array}{ll}0 & 0 \\
\text { in } & 10\end{array}\right|$ & $\mid \begin{array}{cc}O \\
\stackrel{i}{i} & \stackrel{i}{i}\end{array}$ & $\mid \begin{array}{cc}0 & 0 \\
\hat{i} & \dot{P}\end{array}$ & $\left|\begin{array}{cc}0 & 0 \\
i & i\end{array}\right|$ & 웃 & 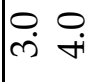 \\
\hline$\sum_{\infty}^{\infty} \widehat{0}$ & \begin{tabular}{l}
0 \\
\multirow{3}{0}{} \\
0 \\
0 \\
$\omega$ \\
0
\end{tabular} & 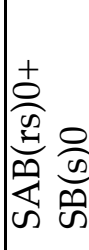 & 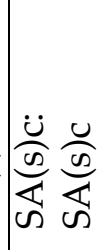 & 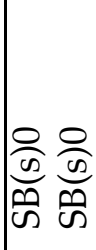 & 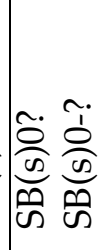 & 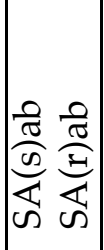 & 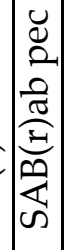 & 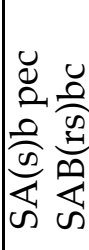 \\
\hline $\begin{array}{l}\text { है } \\
\text { ż }\end{array}$ & $\begin{array}{l}0 \\
N \\
0 \\
0 \\
0 \\
Z\end{array}$ & 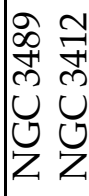 & $\begin{array}{ll}1 & 0 \\
0 & 0 \\
0 & 0 \\
0 & 0 \\
u & 0 \\
0 & 0 \\
Z & Z\end{array}$ & 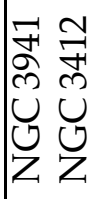 & 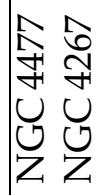 & 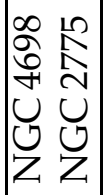 & 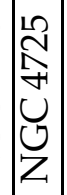 & 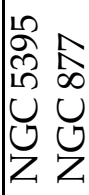 \\
\hline 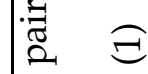 & & $m$ & ले & $\stackrel{19}{m}$ & 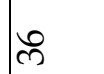 & लि & $\left|\begin{array}{l}\infty \\
\infty\end{array}\right|$ & লু \\
\hline
\end{tabular}




\section{Appendix B}

\section{The VHIKINGS sample}

\section{B.1 The DSS images}

Figs. B.1 to B.4 display the R-band Digital Sky Survey (DSS) images of the 56 galaxies of the VHIKINGS sample. Each Seyfert galaxy is shown on the left of its associated non-active galaxy. Galaxies are listed from the brightest to the faintest nuclear magnitude. An horizontal bar at the bottom right corner of each image shows the comparative spatial resolution for each galaxy. 


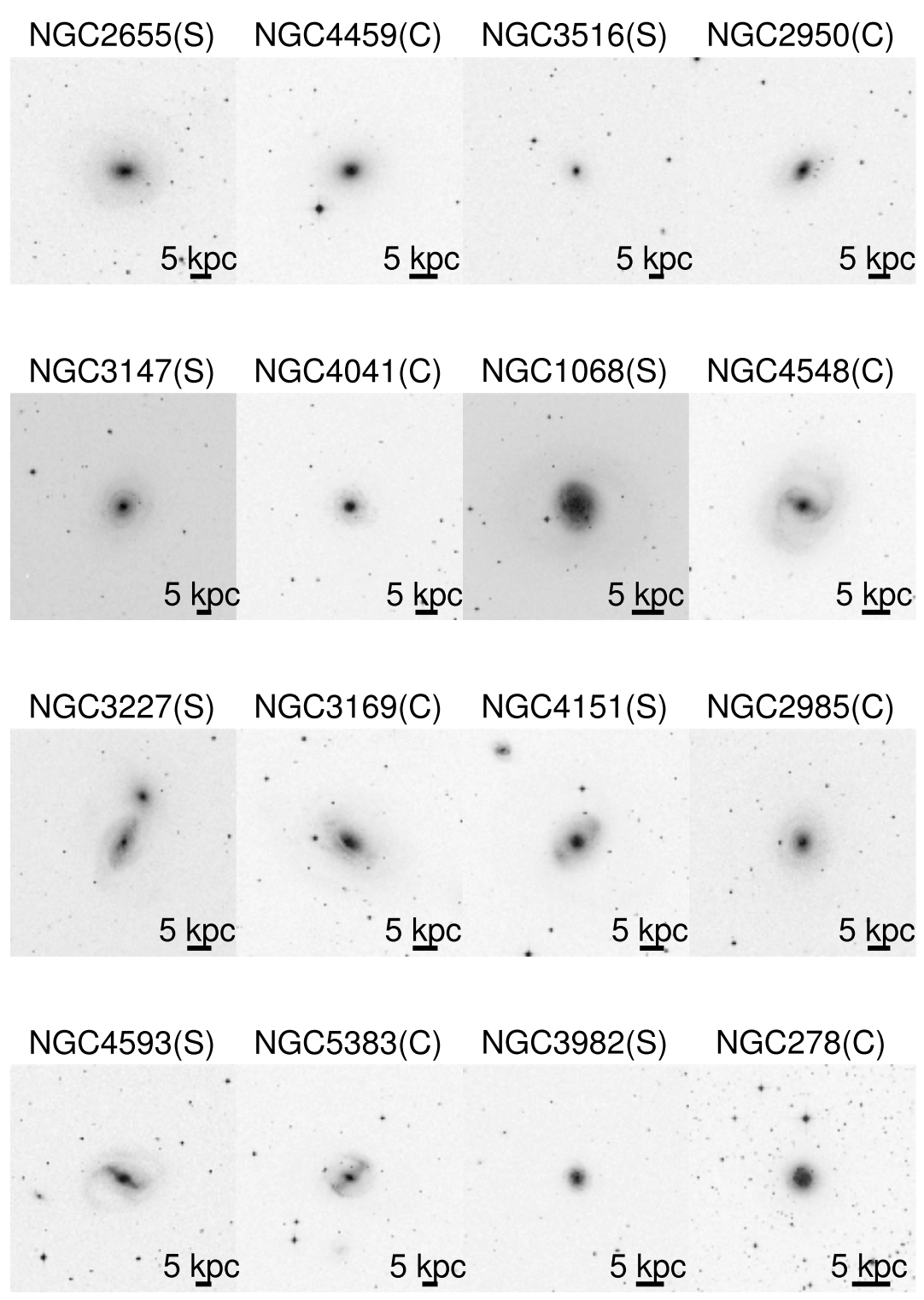

Figure B.1: R-band Digital Sky Survey images of the master sample galaxies. The ' $(S)^{\prime}$ or ' $(C)^{\prime}$ on the right of the object names stand for Seyfert or Control galaxy respectively. Each Seyfert galaxy is displayed on the left of its associated non-active galaxy. The orientation is such that north is up and east is left, each image is $10^{\prime} \times 10^{\prime}$. The horizontal bar located at the bottom right of each panel corresponds to the spatial length of $5 \mathrm{kpc}$. 

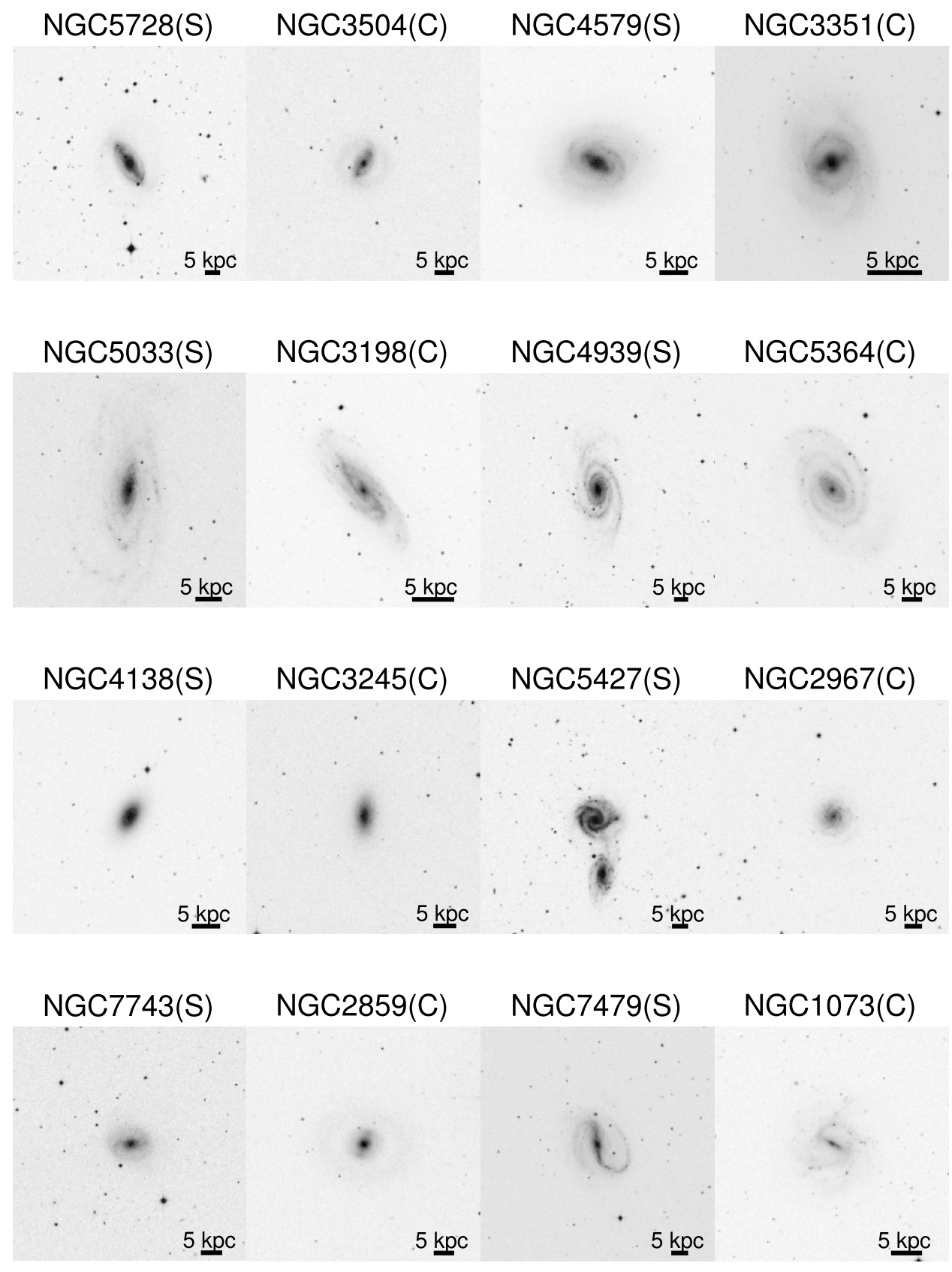

Figure B.2: R-band DSS images of the master sample galaxies, continued. See caption of Fig. B.1 for details. 

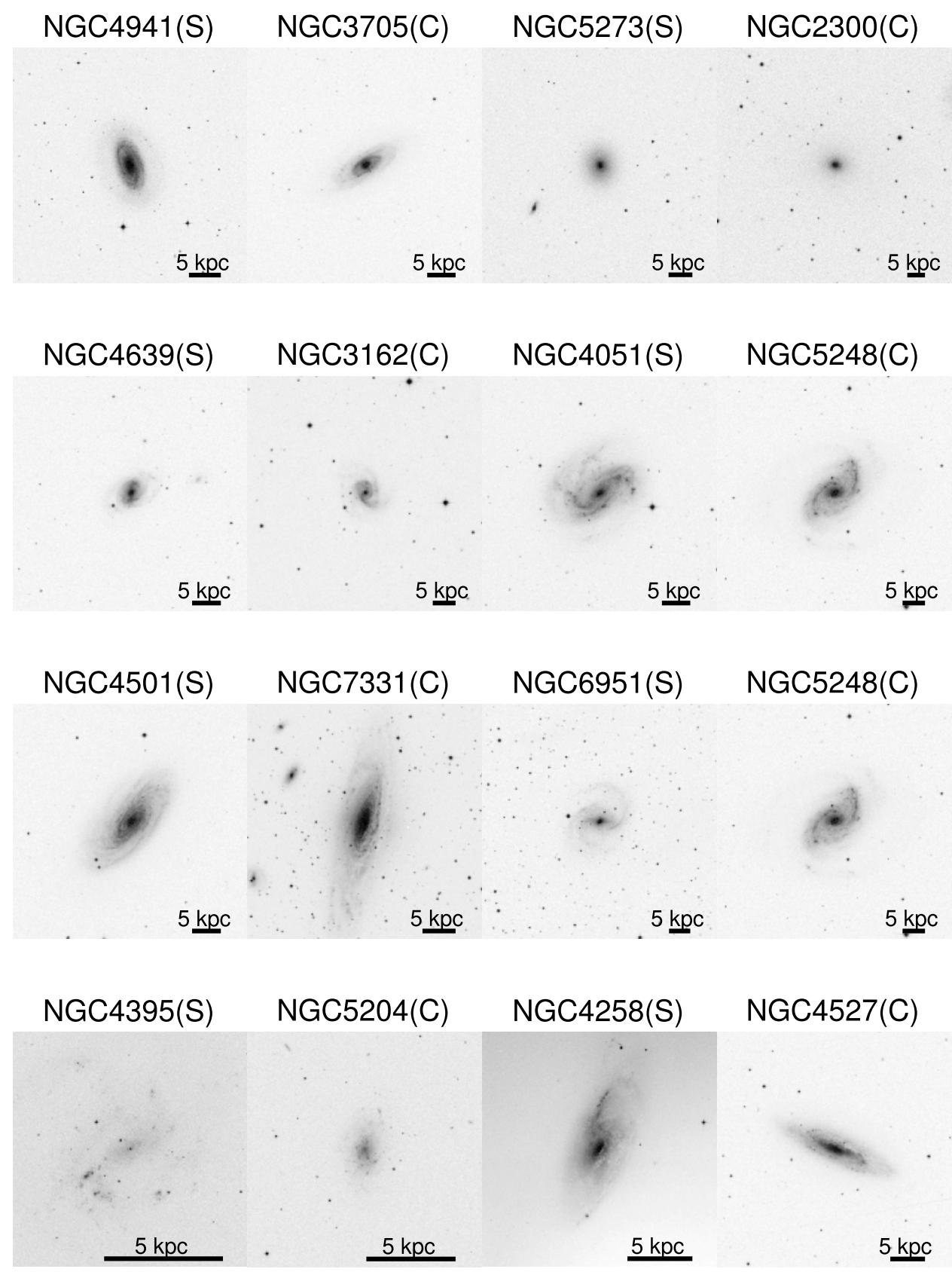

Figure B.3: R-band DSS images of the master sample galaxies, continued. See caption of Fig. B.1 for details. 

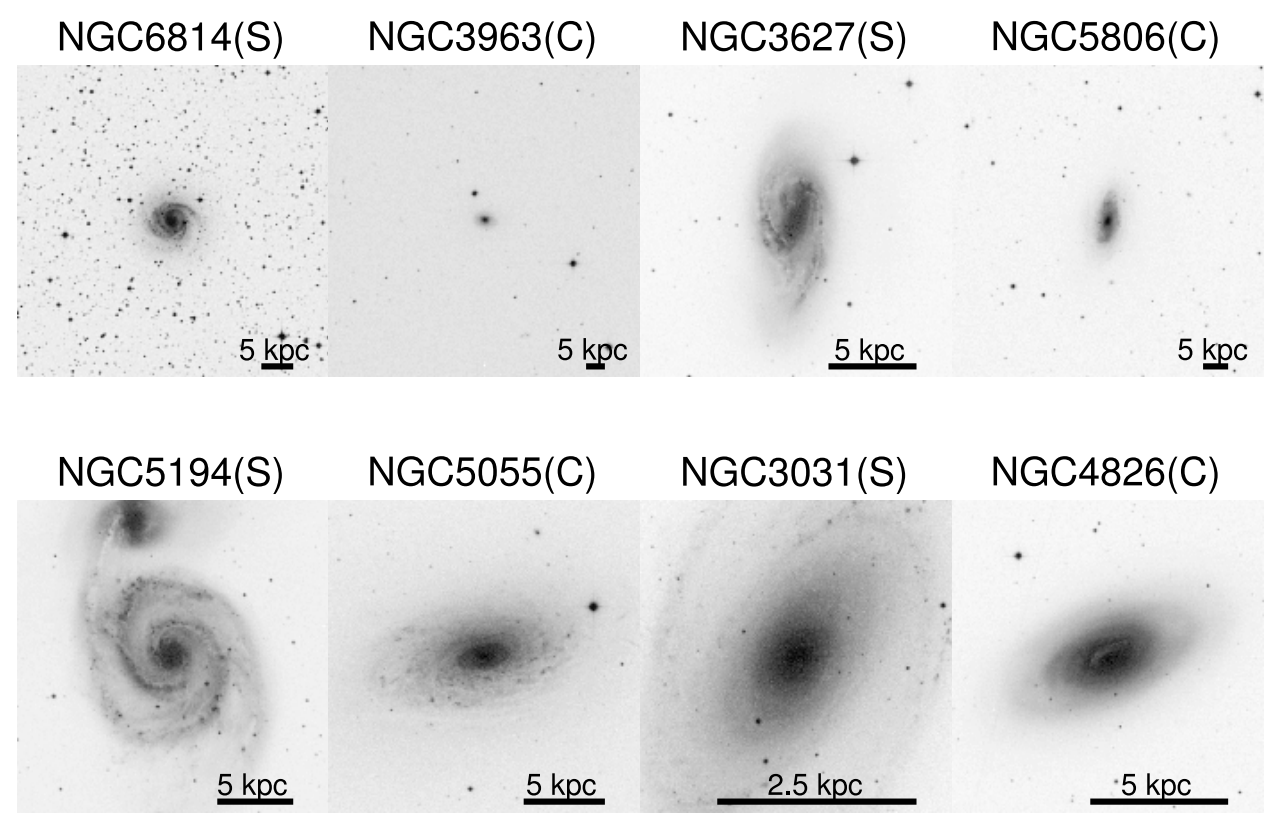

Figure B.4: R-band DSS images of the master sample galaxies, continued. See caption of Fig. B.1 for details. 


\section{B.2 The radio data}

Table B.1 lists the observations parameters of the radio data available for the galaxies of the VHIKINGS survey. 


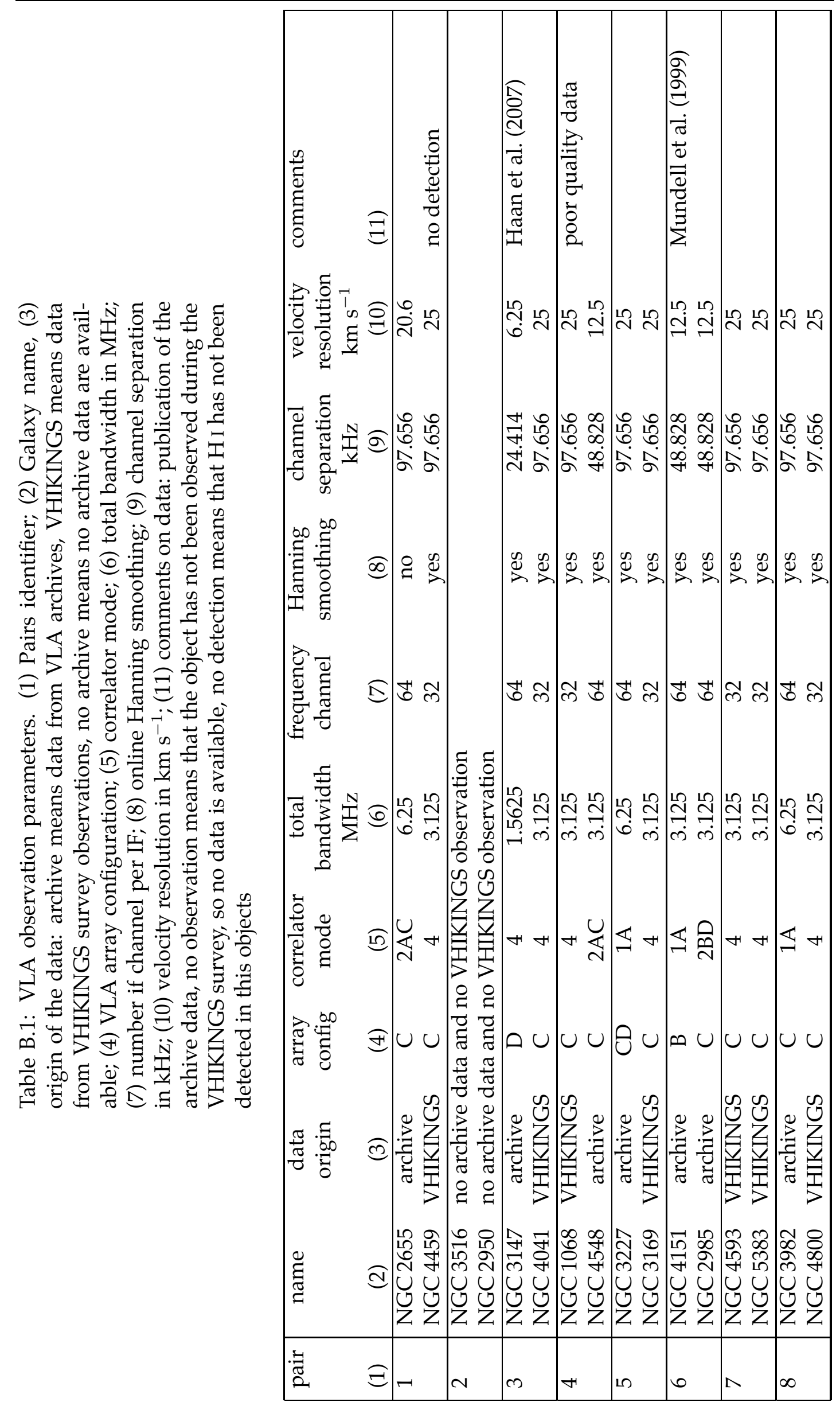




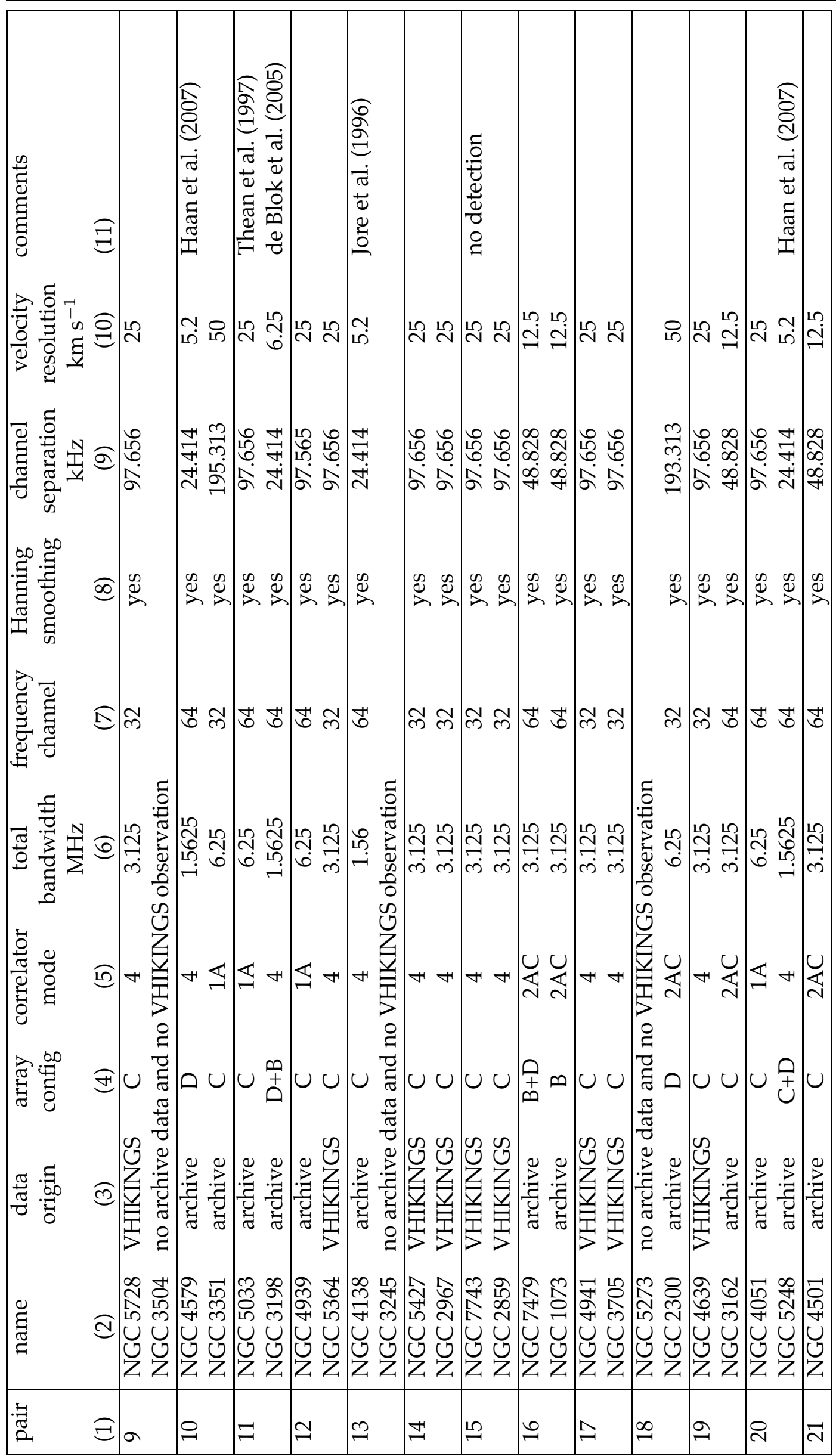




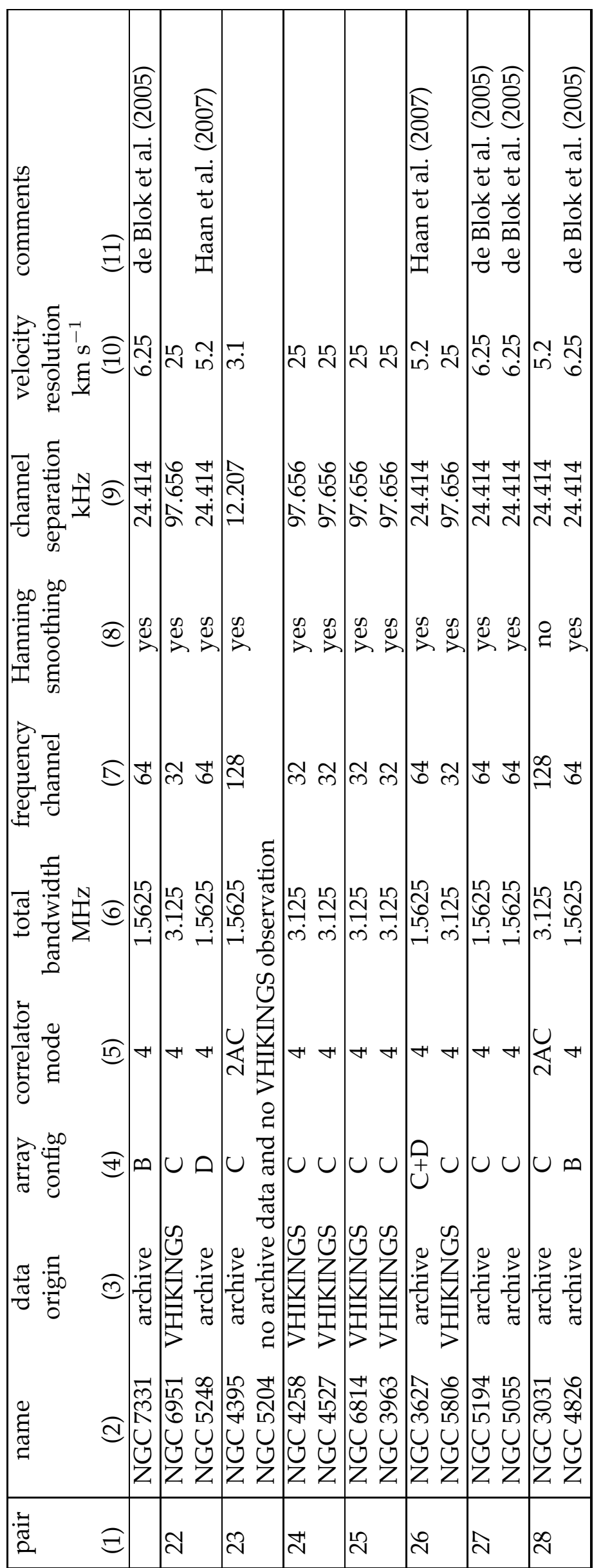




\title{
Appendix C
}

\author{
SAURON
}

\section{moment maps}

\section{C.1 Stellar and ionised gas distribution and kine- matic}

Figs. C.1a to C.1h present the stellar and ionised distribution maps, velocity fields and velocity dispersion, as well as the emission line ratio $[\mathrm{OIII}] / \mathrm{H} \beta$ for the galaxies of the SAURON/Seyfert survey. Each figure presents a pair Seyfert/control galaxies, except for Fig. C.1a which shows NGC1068 and NGC 3227. The ionised gaseous kinematic maps correspond to the [O III] component, except for NGC 4459, for which $\mathrm{H} \beta$ (see Sec. 4.1). 

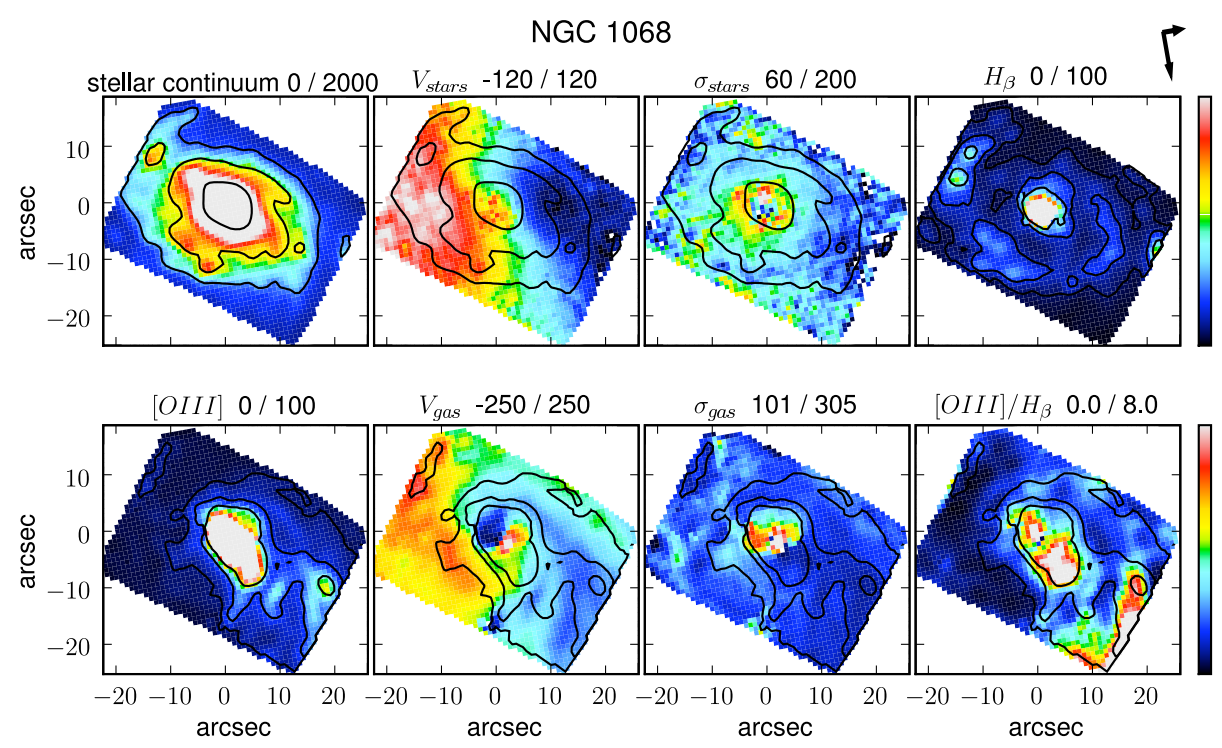

NGC 3227
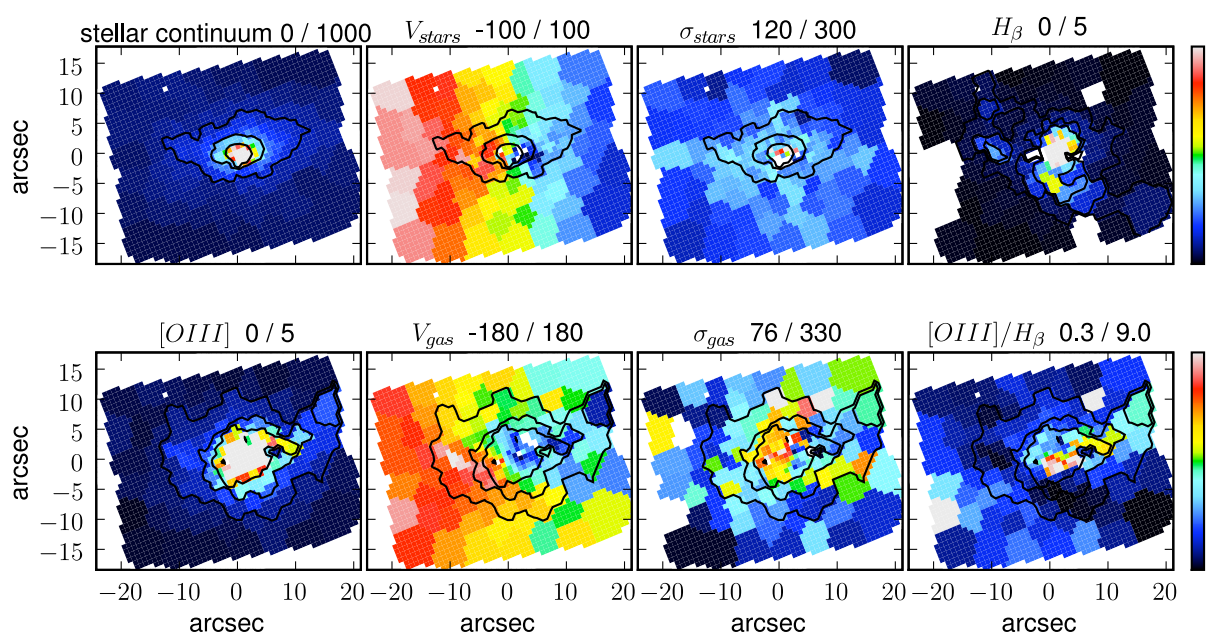

Figure C.1a: SAURON maps for the Seyfert galaxies NGC 1068 (top panels) and NGC 3227 (bottom panels). For each galaxy the first row shows (from left to right) the reconstructed continuum map, the stellar velocity field, the stellar velocity dispersion map and $\mathrm{H} \beta$ intensity distribution. The second row consists of the [O III] intensity velocity and velocity dispersion maps and the line ratio [O III] / $\mathrm{H} \beta$ map. All the intensity maps are in units of $10^{-16} \mathrm{erg} \mathrm{s}^{-1} \mathrm{~cm}^{-2}$. The stellar and emission lines intensity maps are overlaid on the stellar and ionised gas kinematics. Velocities and velocity dispersions are in $\mathrm{km} \mathrm{s}^{-1}$. The colour scale is shown on the right hand side and the cut levels are indicated in the top right corner of each map. All the maps are orientated so that the photometric major-axis of the galaxy is on the x-axis. The long and short arrows on the right of each galaxy name show the north and the east directions, respectively. 

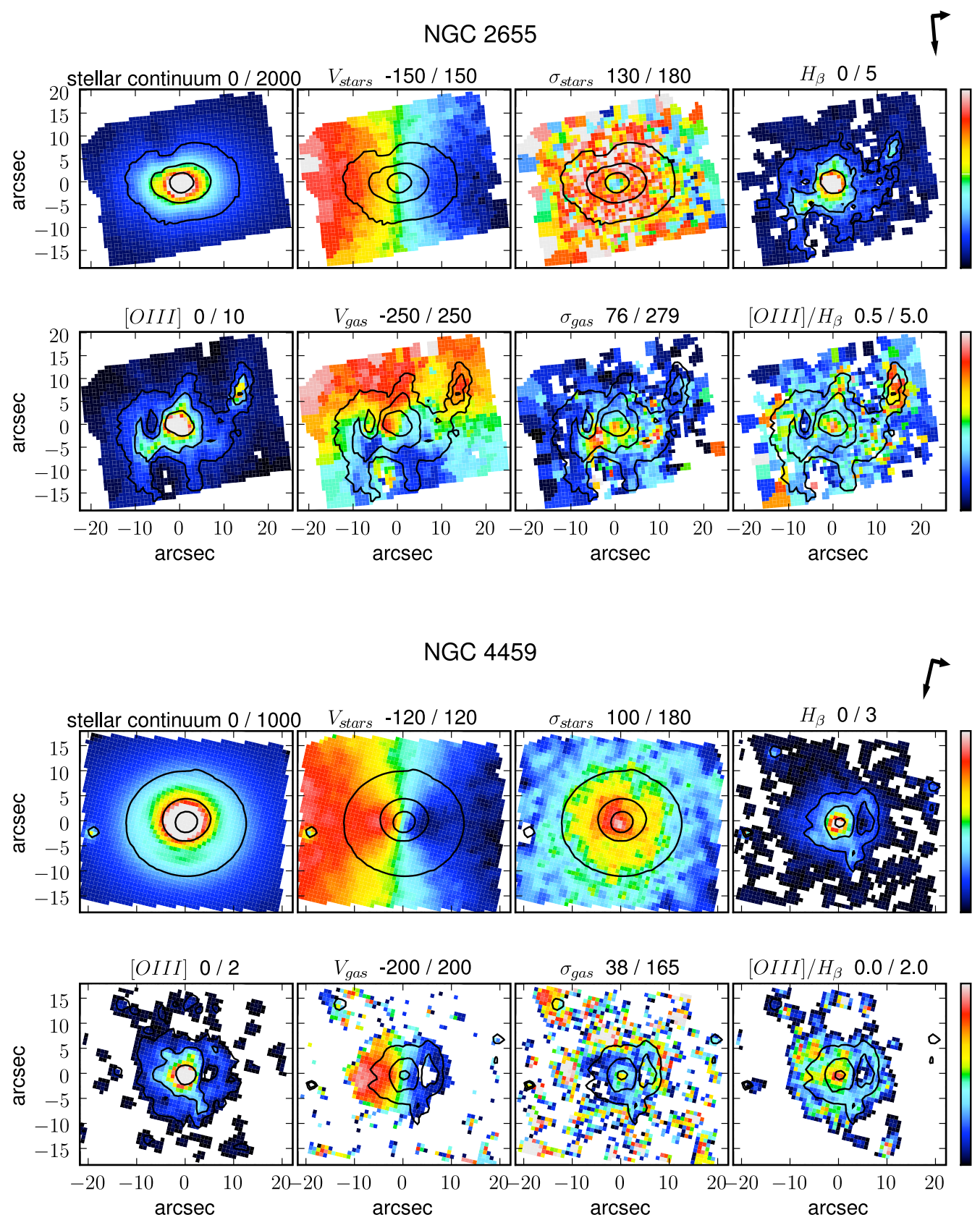

Figure C.1b: SAURON maps for NGC2655 (top panels) and its control NGC 4459 (bottom panels). See caption of Fig. C.1a for details. 
NGC 3627
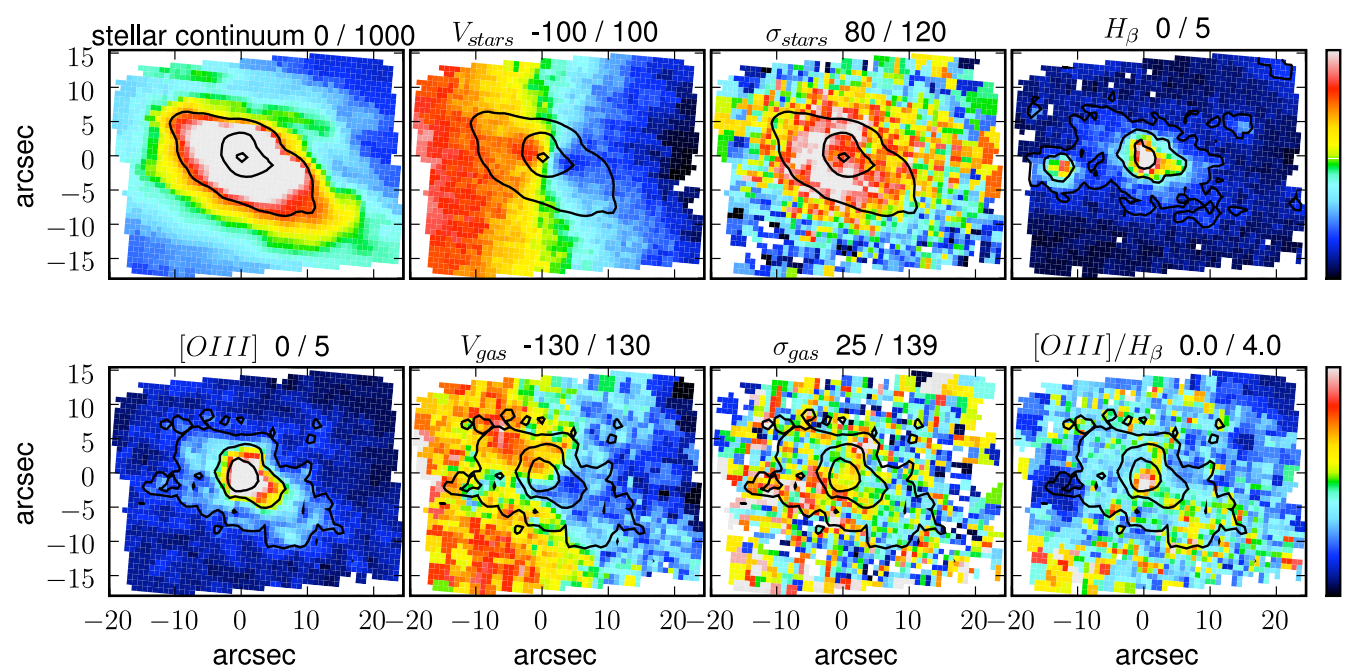

NGC 5806
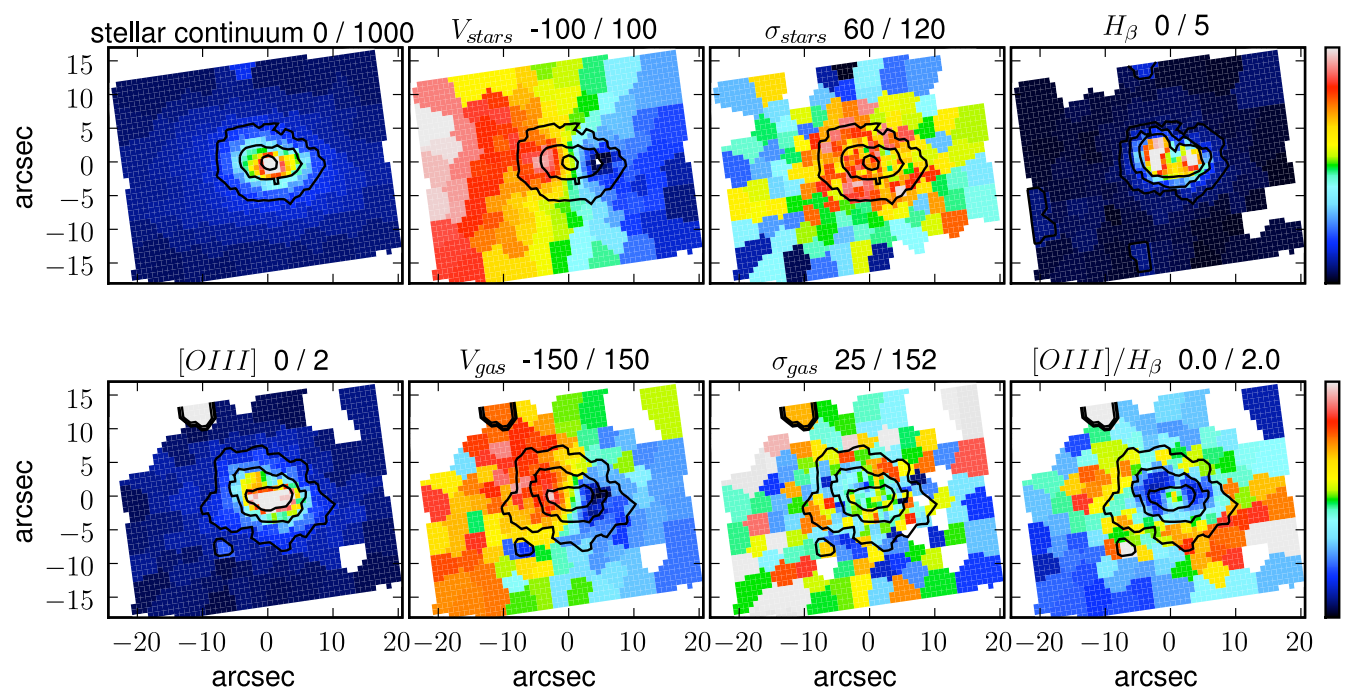

Figure C.1c: SAURON maps for NGC 3627 (top panels) and its control NGC 5806 (bottom panels). See caption of Fig. C.1a for details. 

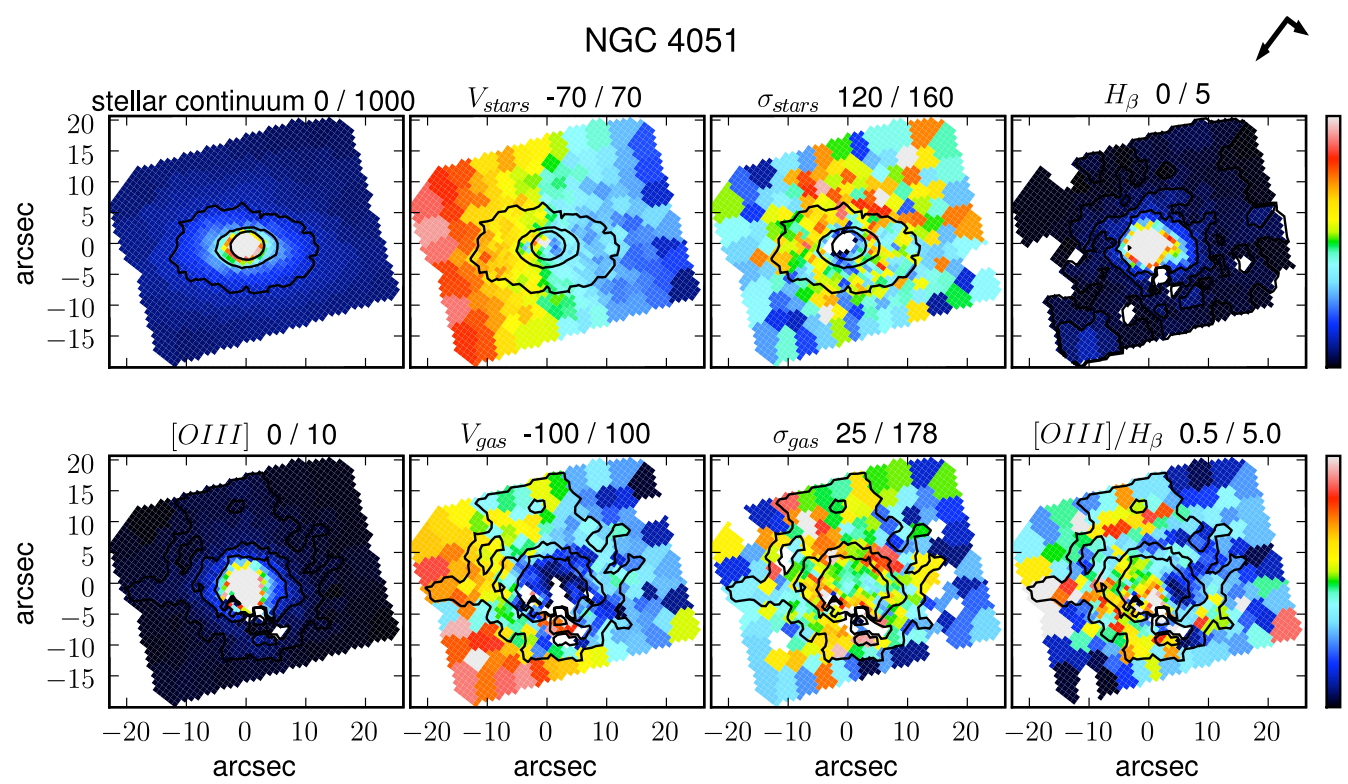

NGC 5248
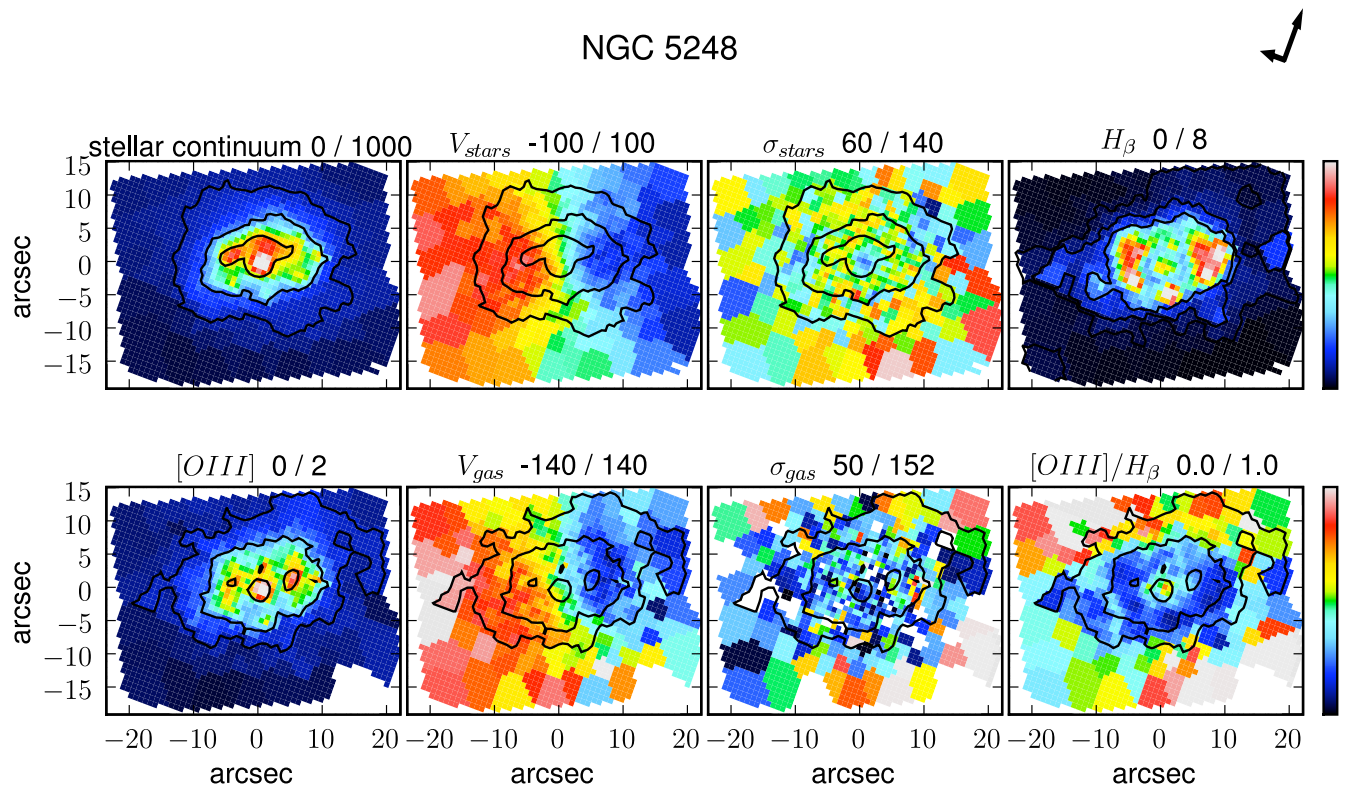

Figure C.1d: SAURON maps for NGC4051 (top panels) and its control NGC 5248 (bottom panels). See caption of Fig. C.1a for details. 

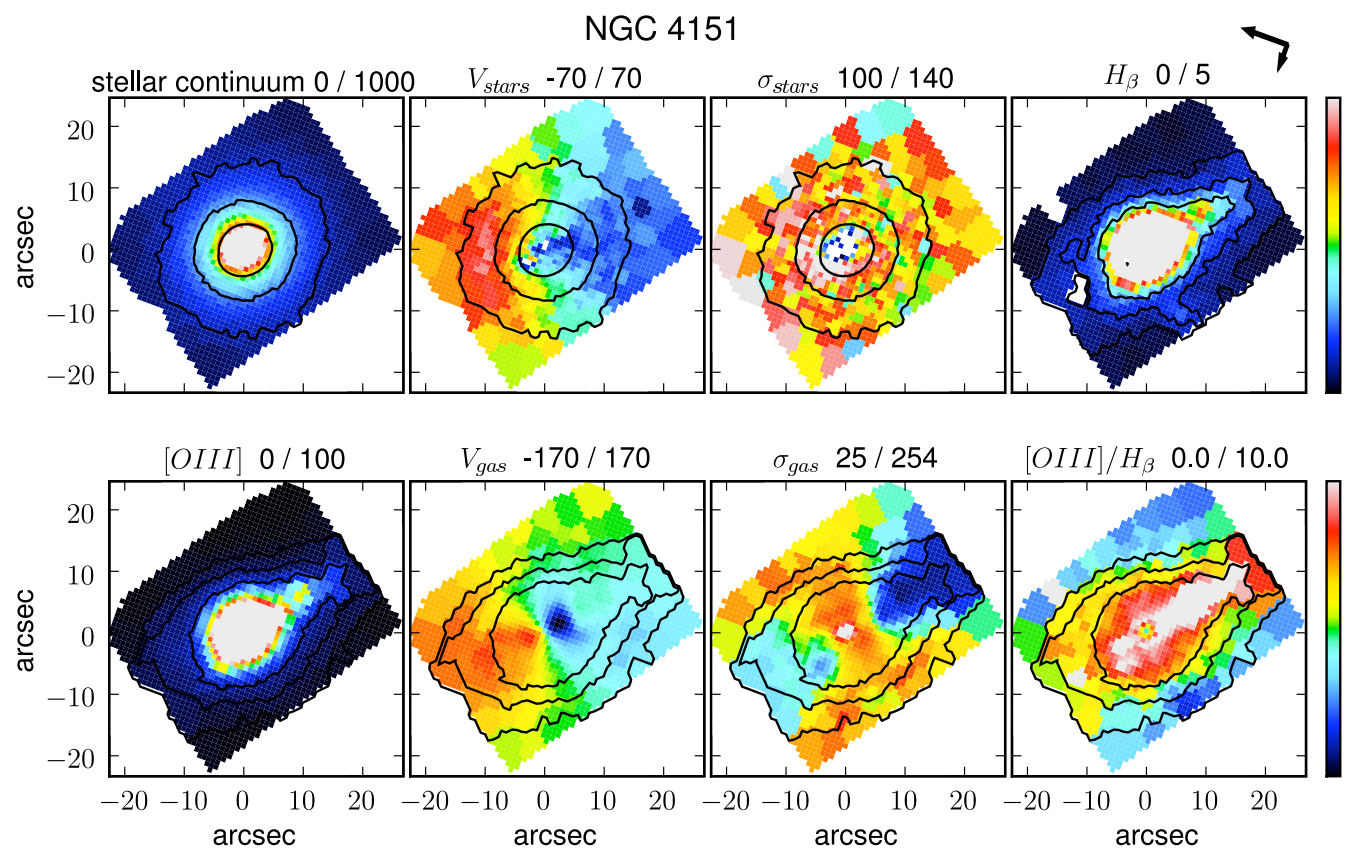

NGC 2985
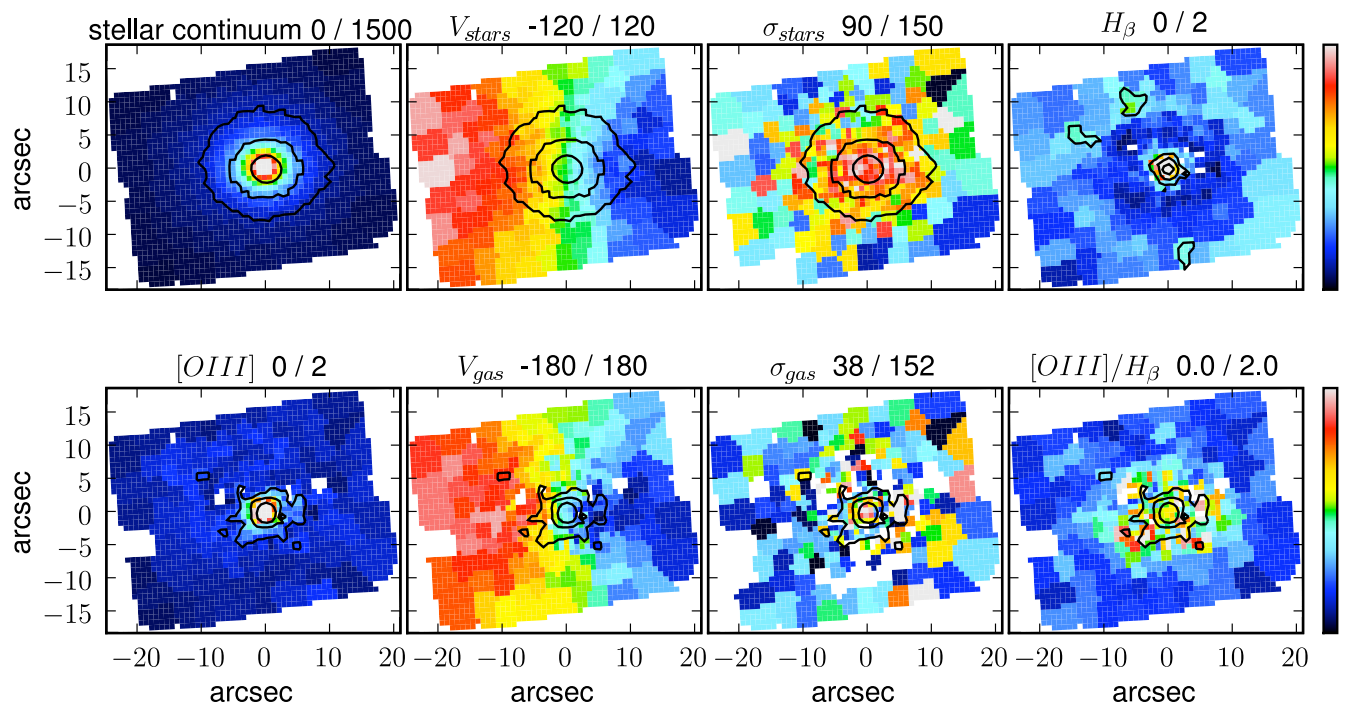

Figure C.1e: SAURON maps for NGC 4151 (top panels) and its control NGC 2985 (bottom panels). See caption of Fig. C.1a for details. 

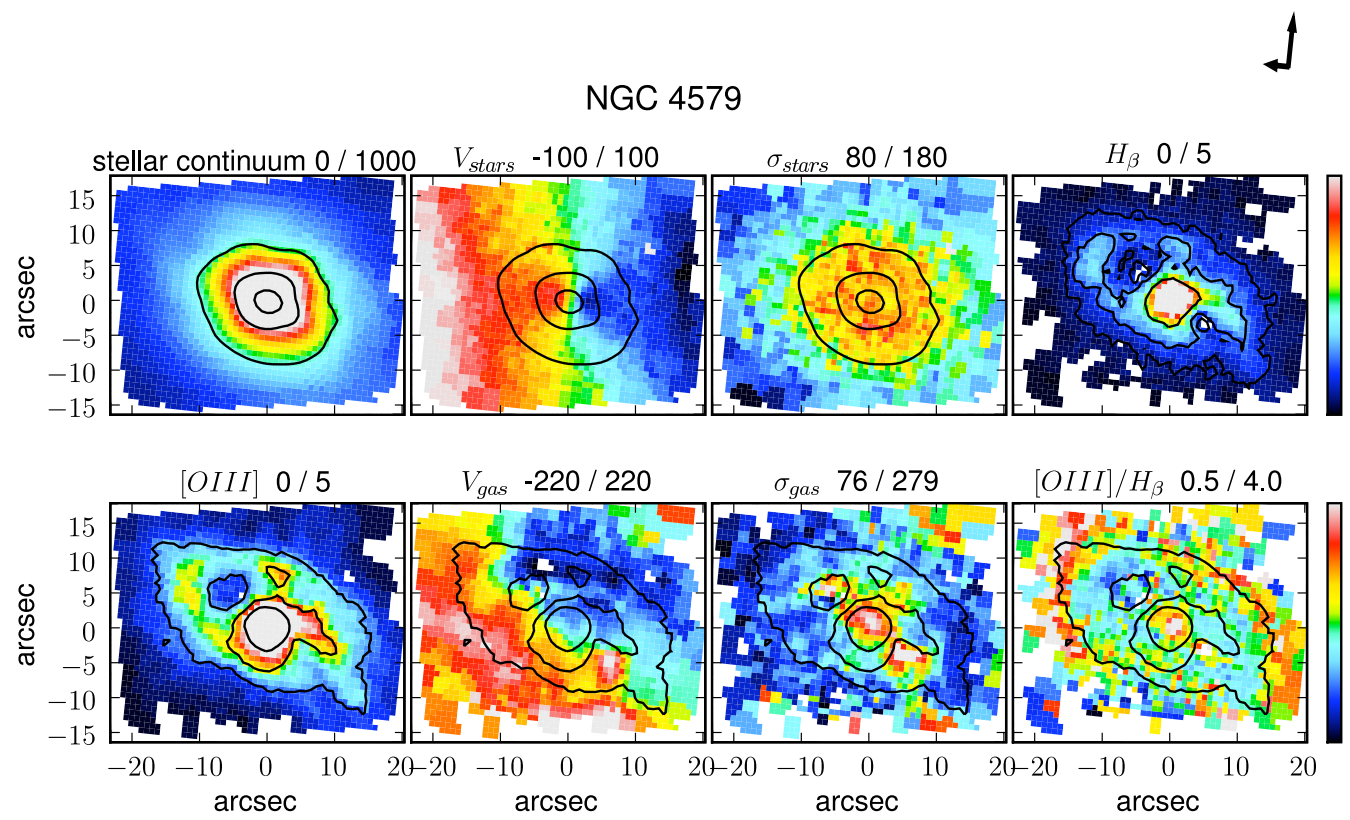

NGC 3351
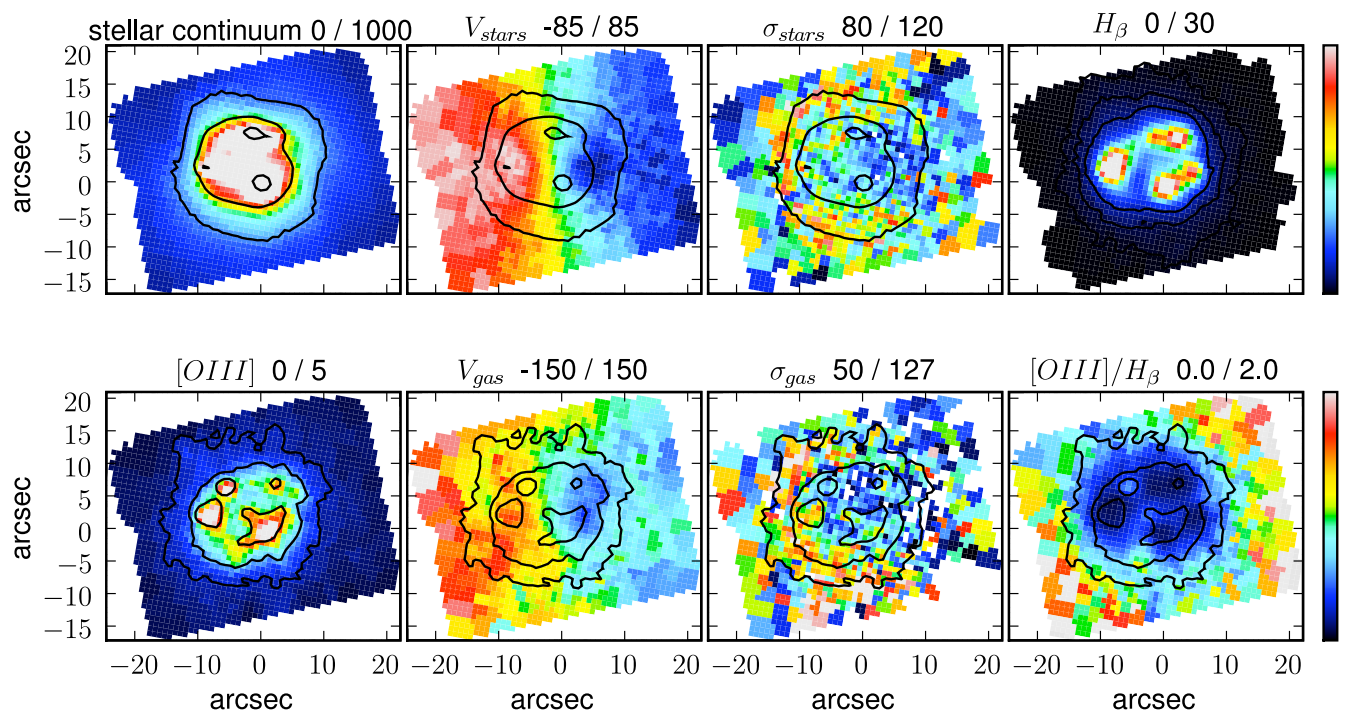

Figure C.1f: SAURON maps for NGC 4579 (top panels) and its control NGC 3351 (bottom panels). See caption of Fig. C.1a for details. 
NGC 5194
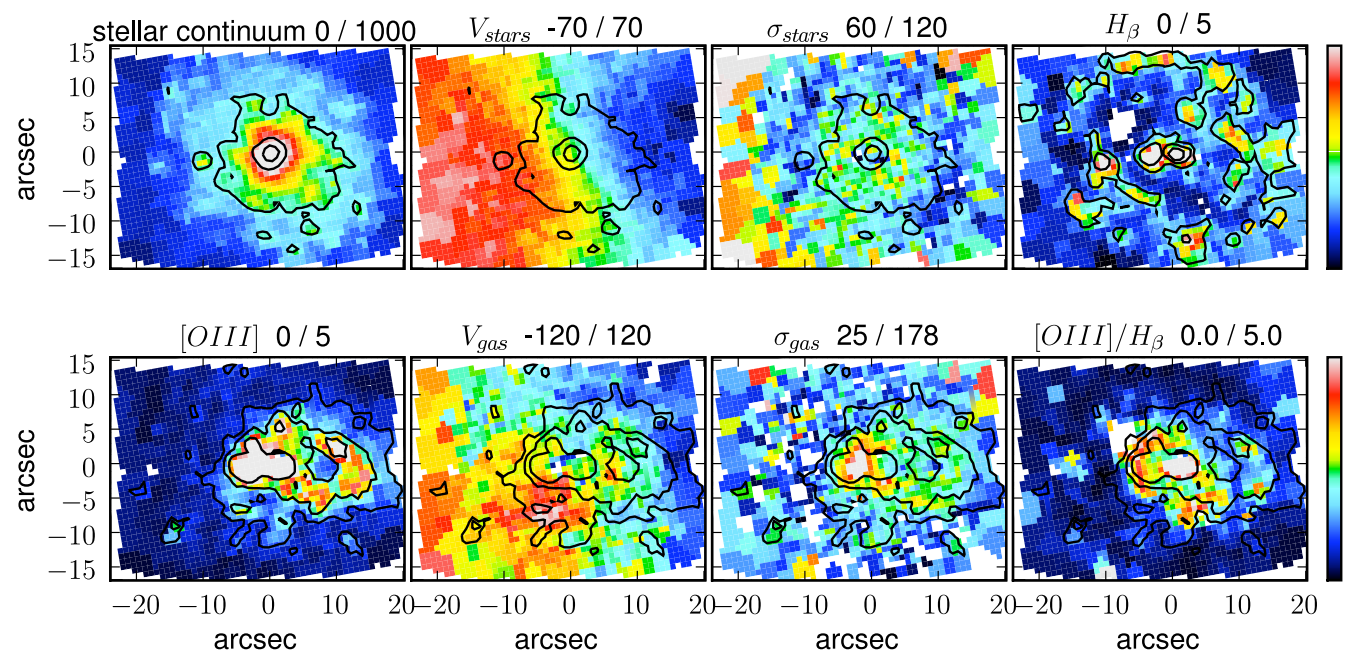

NGC 5055
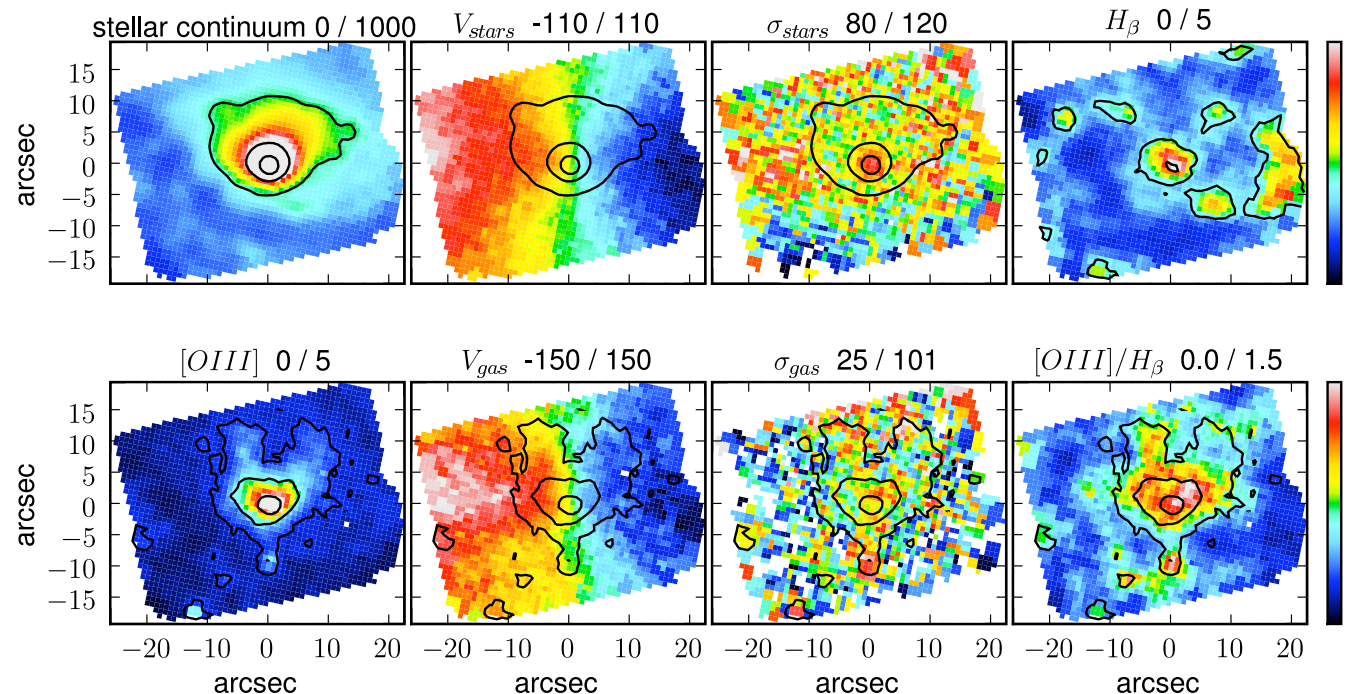

Figure C.1g: SAURON maps for NGC5194 (top panels) and its control NGC 5055 (bottom panels). See caption of Fig. C.1a for details. 
NGC 6951
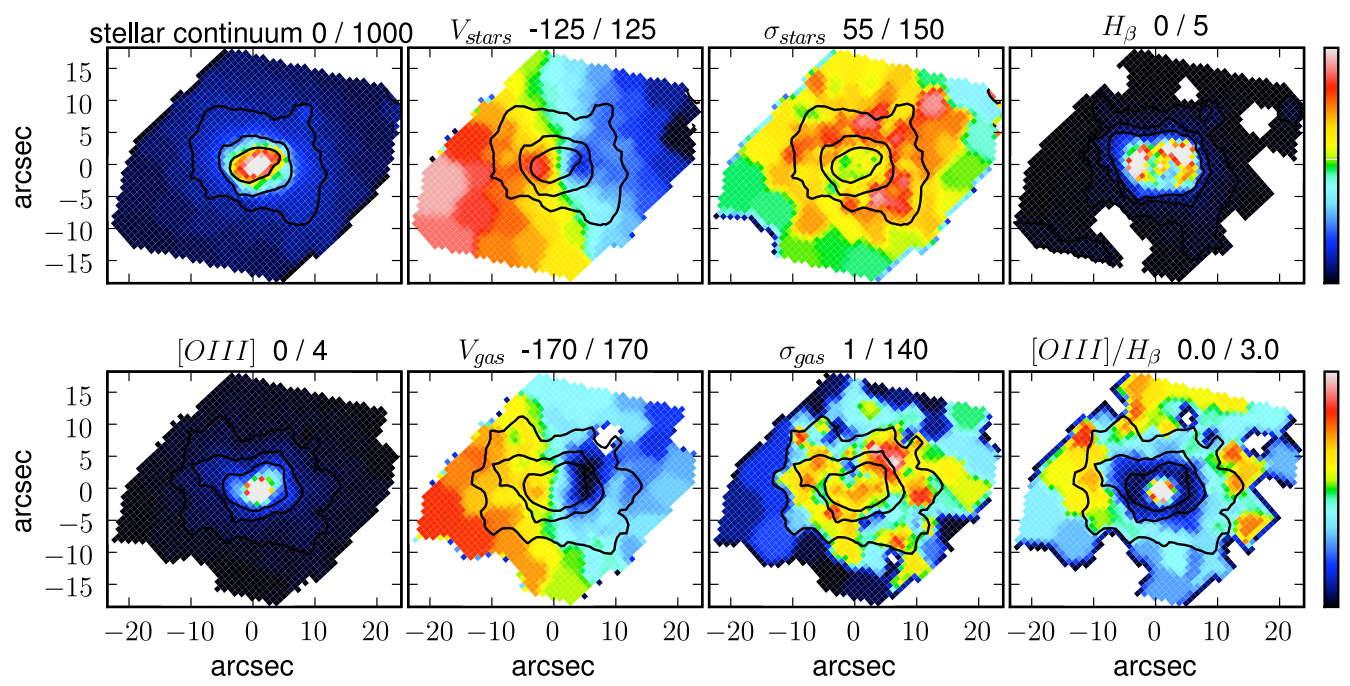

NGC 5248
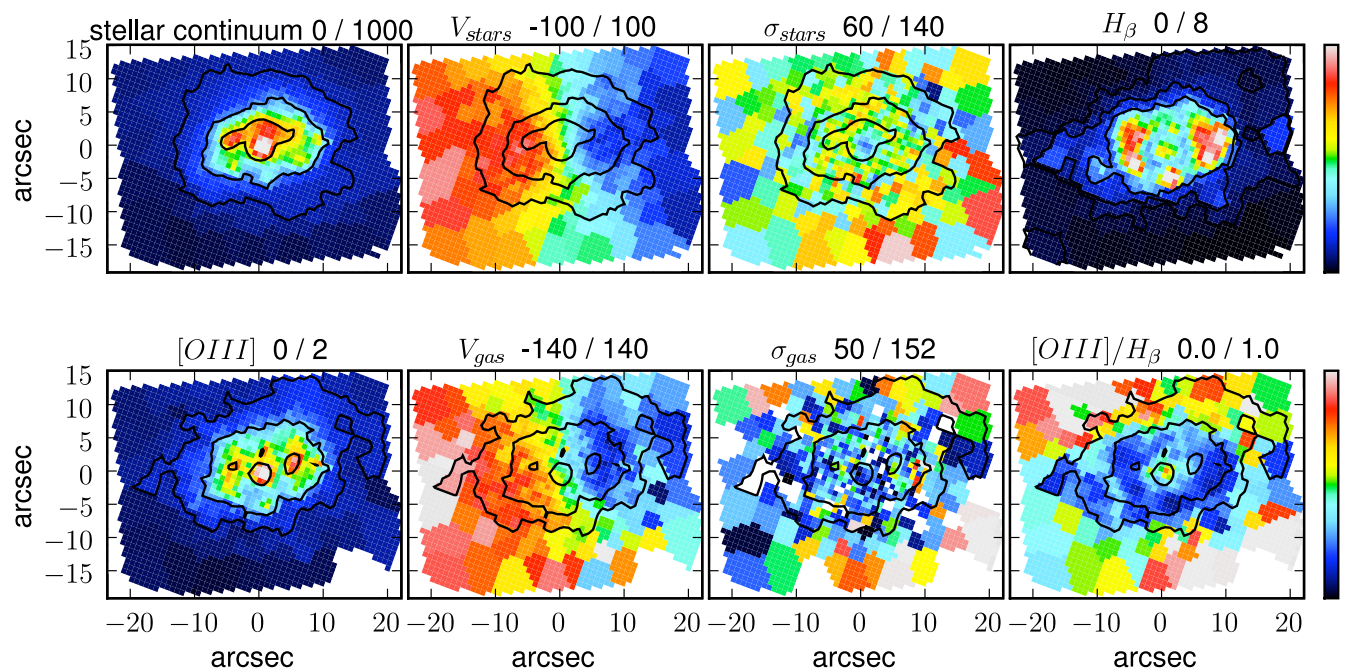

Figure C.1h: SAURON maps for NGC6951 (top panels) and its control NGC 5248 (bottom panels). See caption of Fig. C.1a for details. 


\section{C.2 $h_{3}$ and $h_{4}$ maps}

Figs. C.2a and C.2b present the two-dimensional maps of the $h_{3}$ and $h_{4}$ coefficients, corresponding to the stellar kinematics fit (see Sec. 3.2.2). 

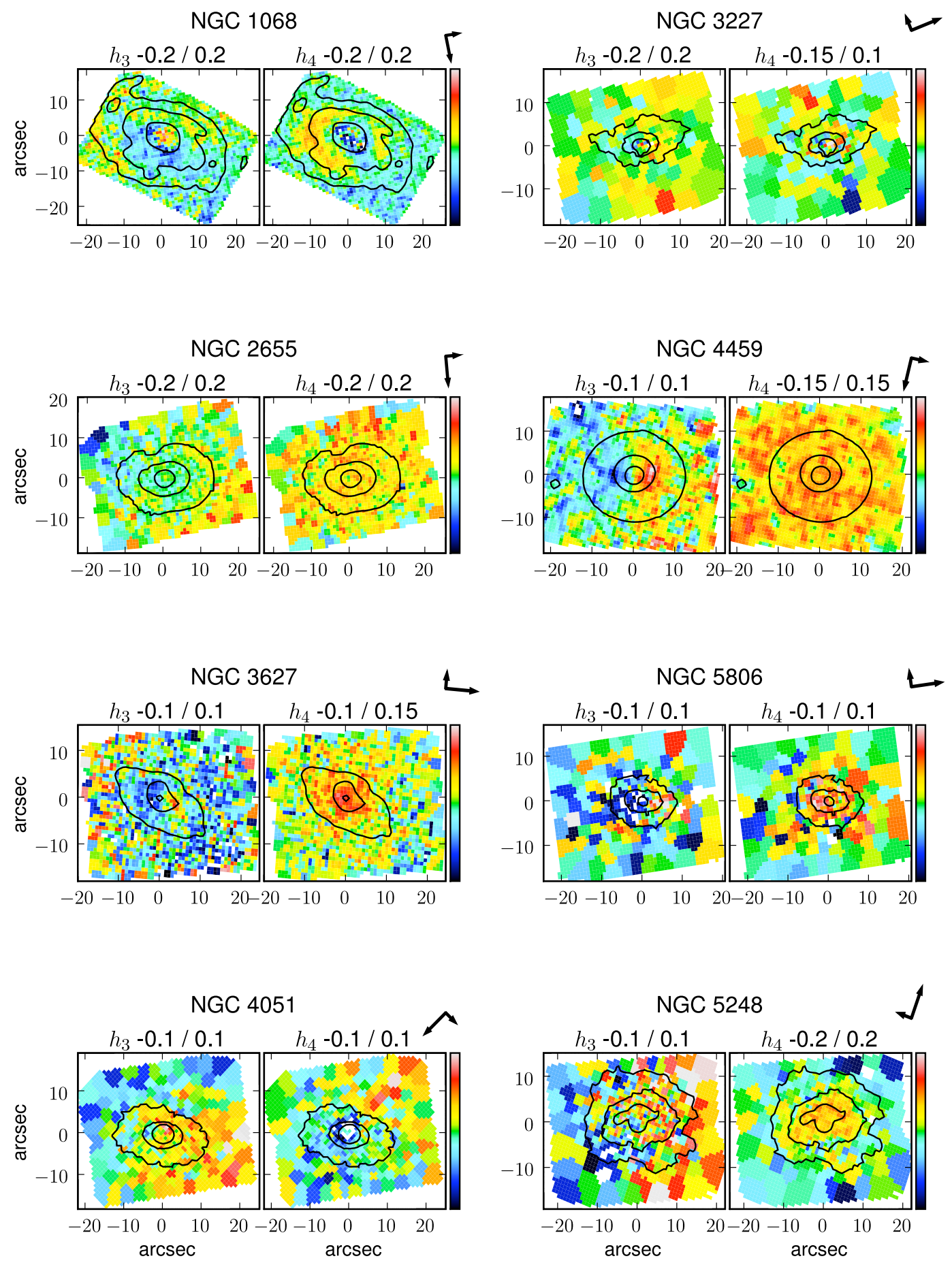

Figure C.2a: $h_{3}$ and $h_{4}$ maps. Each row presents a pair : Seyfert on the left, Control on the right, except for NGC 1068 and NGC 3227 which are shown together in the first row. The colour scale is shown on the right hand side and the cut levels are indicated in the top right corner of each map.The long and short arrows on the right of each galaxy name show the north and the east directions, respectively. 

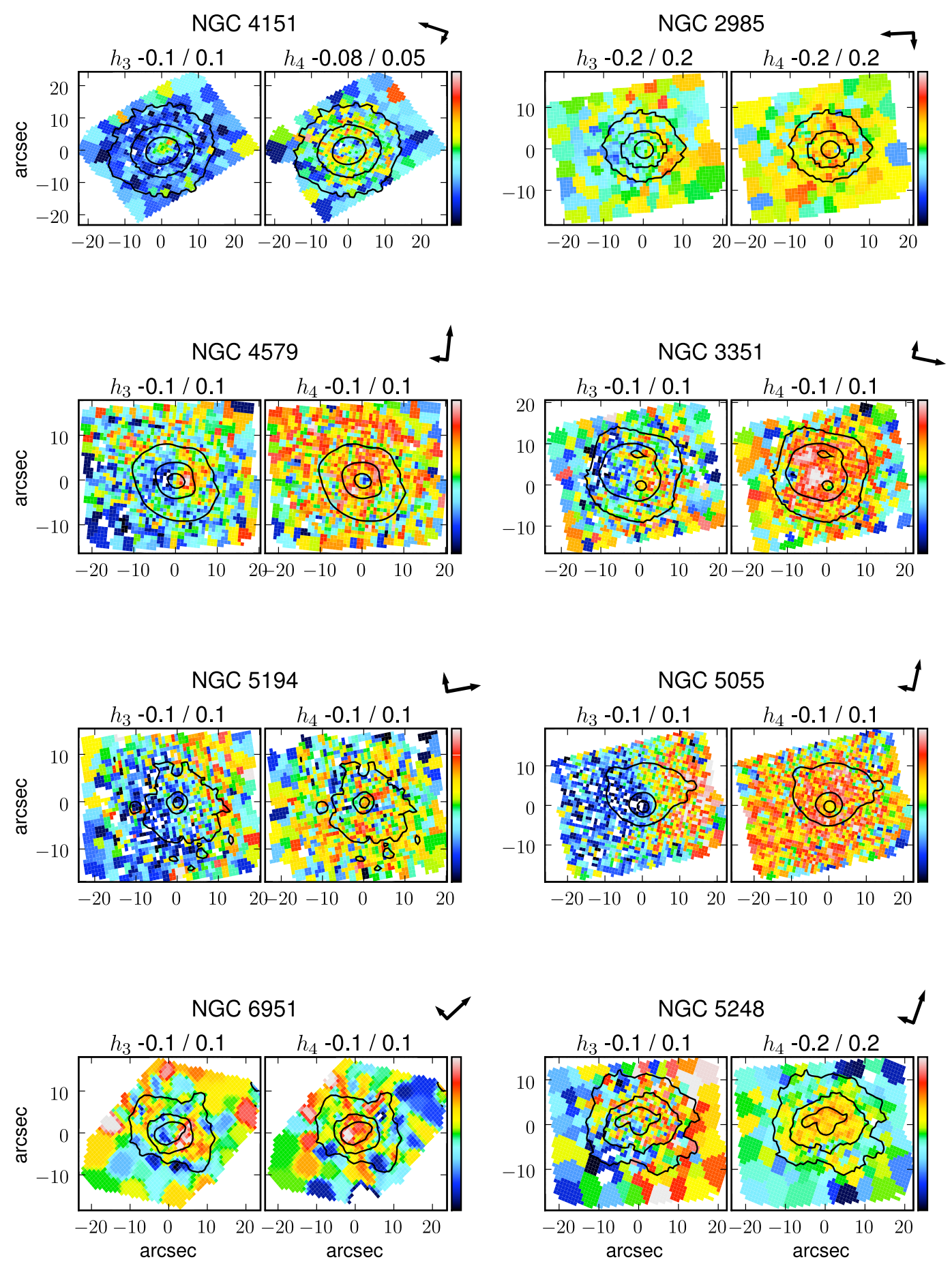

Figure C.2b: Fig. C.2a continued. 


\section{C.3 $\mathbf{H} \beta$ kinematic maps}

Figs. C.3a and C.3b present the velocity and velocity dispersion maps of our galaxies observed with SAURON. For NGC 4459, the maps corresponding to [O III] are presented (see Sec. 4.1). 

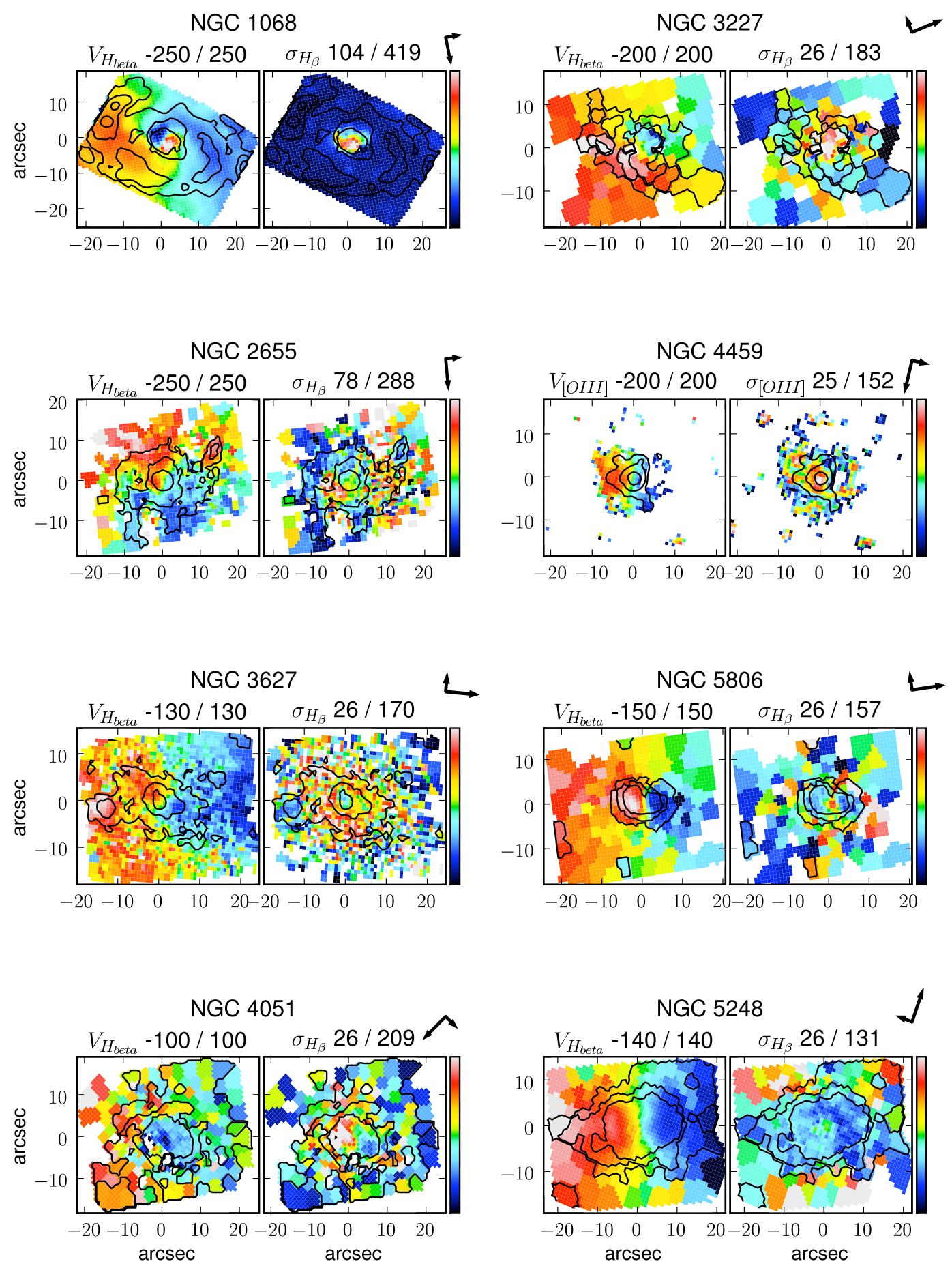

Figure C.3a: Velocity and velocity dispersion of $\mathrm{H} \beta$ emission lines. Each row presents a pair : Seyfert on the left, Control on the right, except for NGC 1068 and NGC 3227 which are shown together in the first row. For NGC 4459, the [O III] velocity and velocity dispersion maps are shown. All the maps are orientated so that the photometric major-axis of the galaxy is on the x-axis. The units are in $\mathrm{km} \mathrm{s}^{-1}$. The colour scale is shown on the right hand side and the cut levels are indicated in the top right corner of each map. The long and short arrows on the right of each galaxy name show the north and the east directions, respectively. 

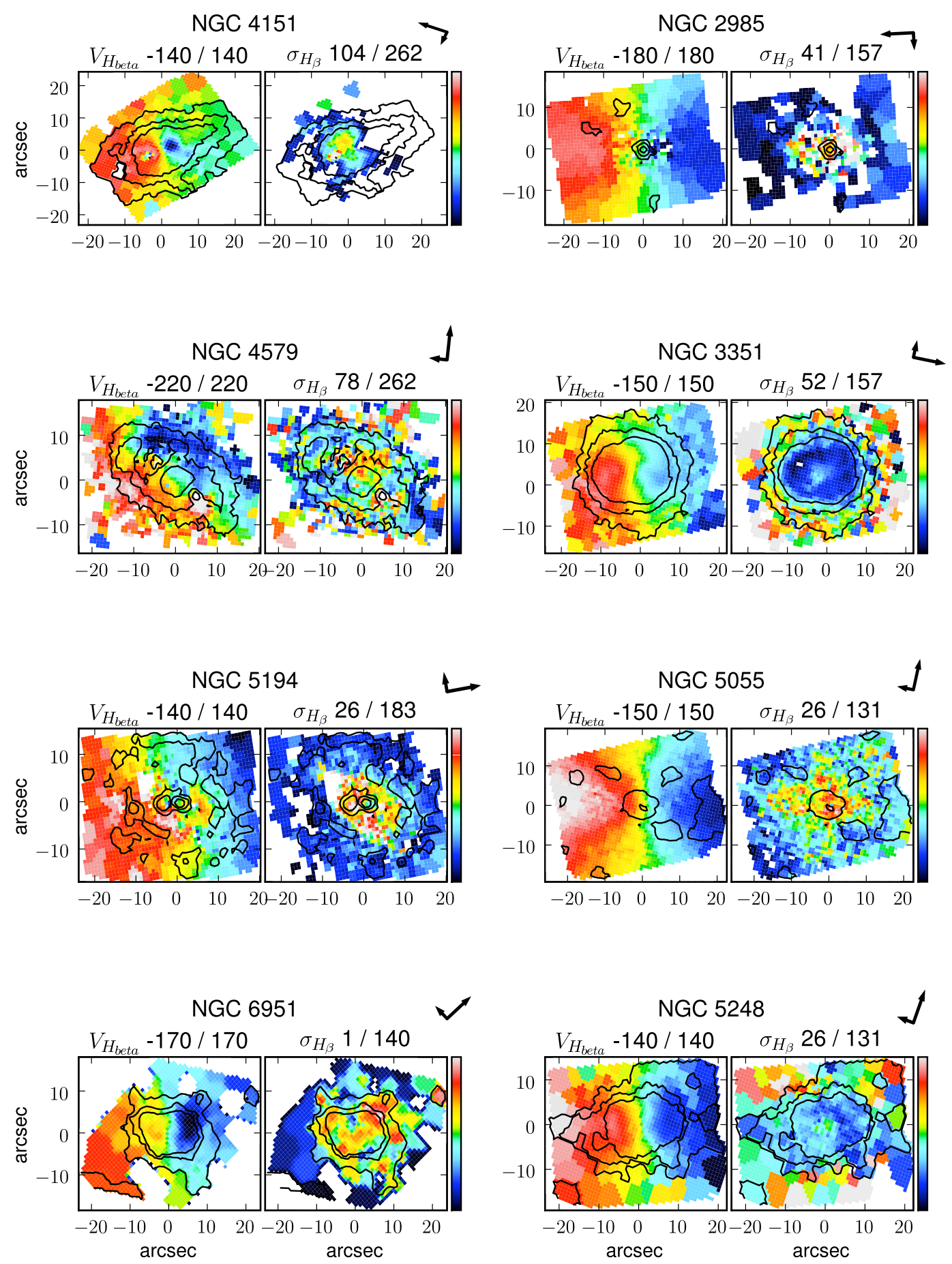

Figure C.3b: Fig. C.3a continued. 


\section{Appendix D}

\section{HI moment maps}

Figs. D.1a to D.1f present the H I moment maps (distribution and velocity fields) along with the R-band DSS image at the same spatial scales for the galaxies of the VHIKINGS survey, for which data reduction has already been completed. The '(S)' or ' $(\mathrm{C})^{\prime}$ on the right of the galaxy name correspond to Seyfert and Control, non-active galaxy, respectively. Like the optical data (Appendix C), the galaxies are presented following the pairs Seyfert/non-active galaxies. When H I data is missing for a galaxy, a blank space is left for that galaxy. 


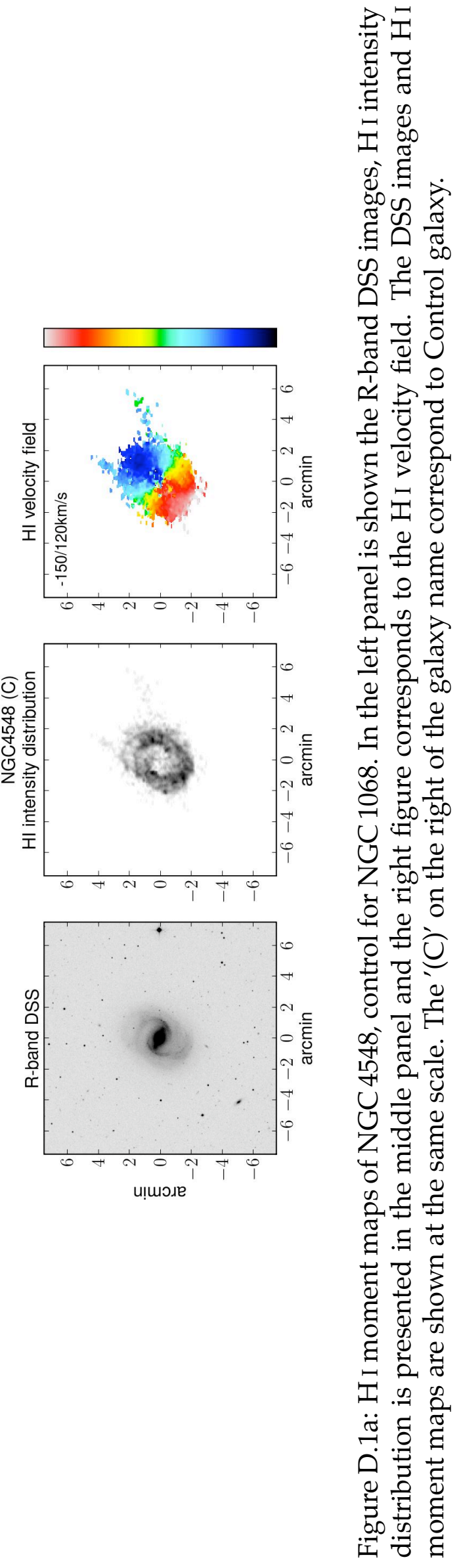




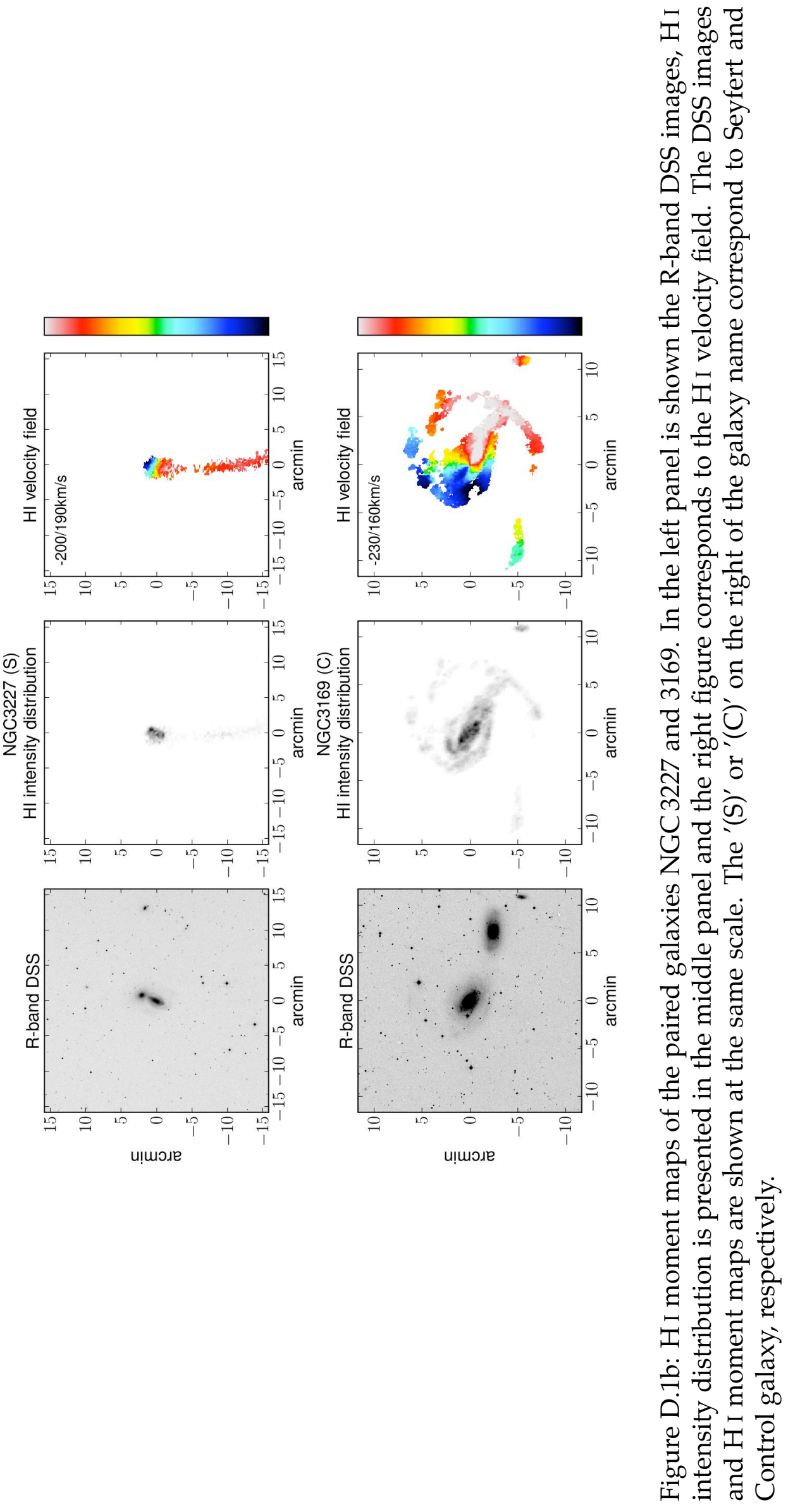




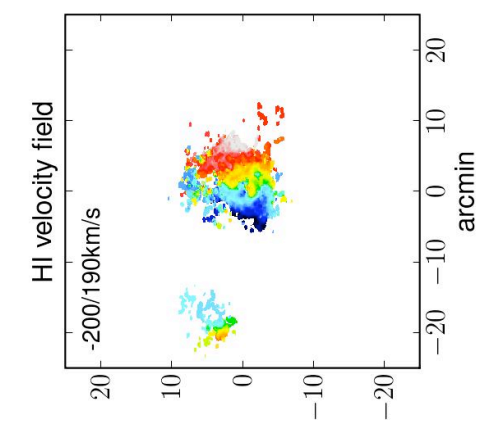

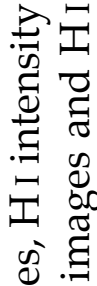

๘心

की

무

उ क

숭ㅇ

ญ. ఫِّ

द के

का

.

ฮ월

ีㅡㅇ 은

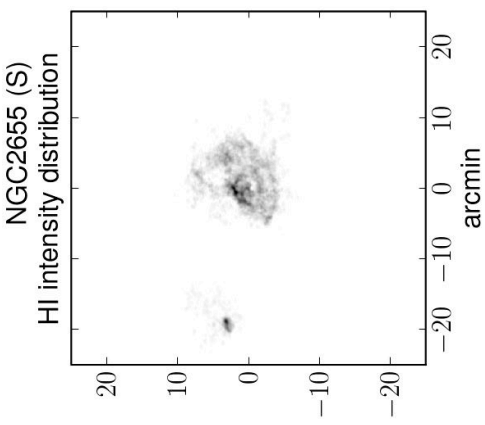

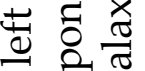

() के 0

픙

당

กิ

N 50.00

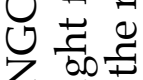

के 0 0

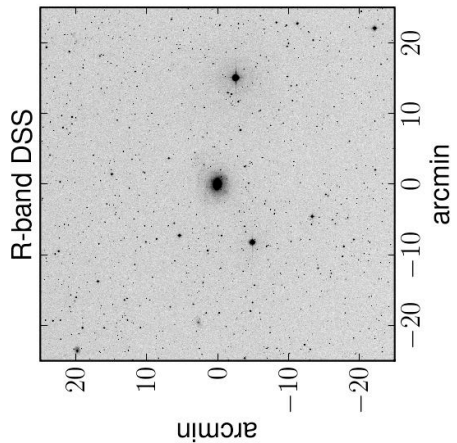

舫产

๙

드

Чٓํำ

के

全. है

00

क \&

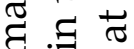

元焉

है क

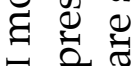

I

¿ ह

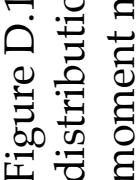




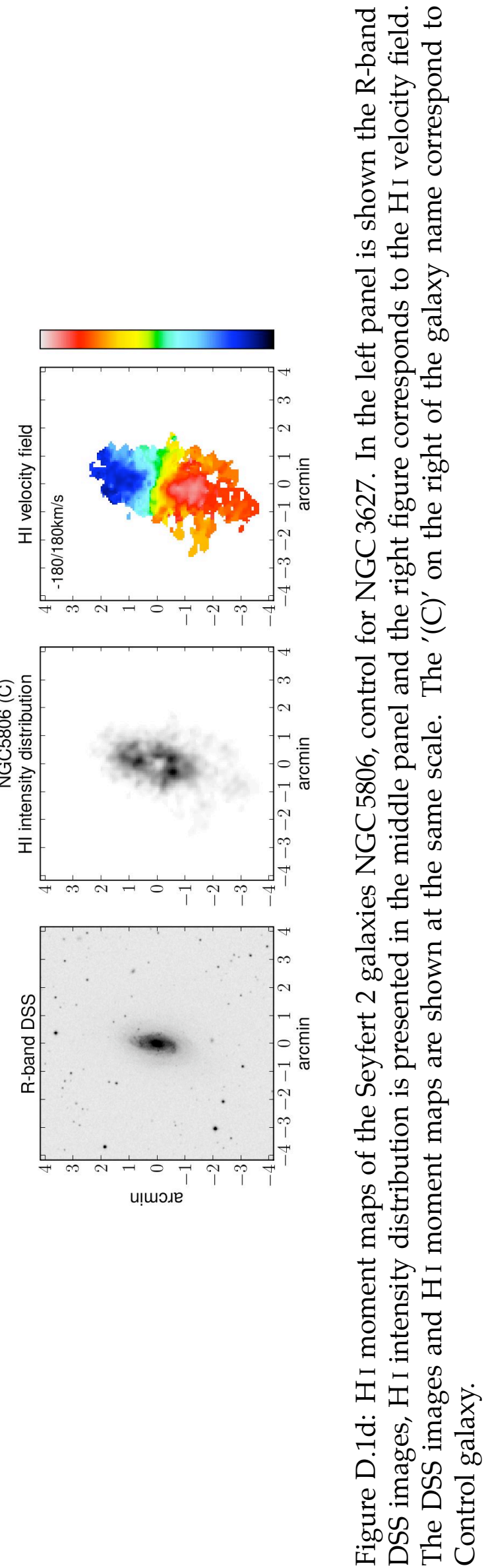




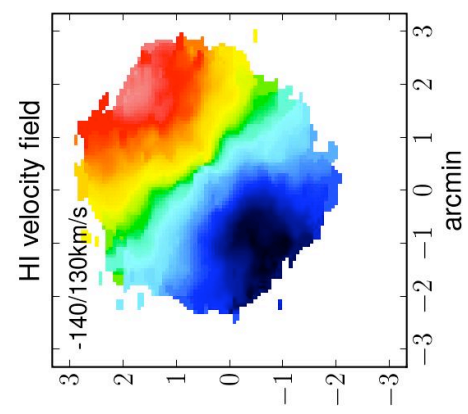

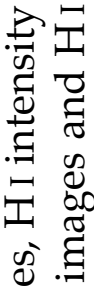

שू

की

E

तี बे

$\therefore$ 구

ญ. ఫِّ

58 के

도

क 08

‡

워 즈

눙

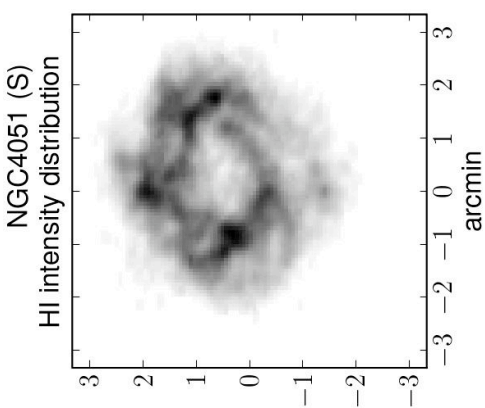

tै

व के

$\mp$ ¿

트

돈

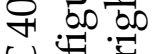

$\circlearrowleft \frac{1}{0}$

\& $\Xi$ ธี

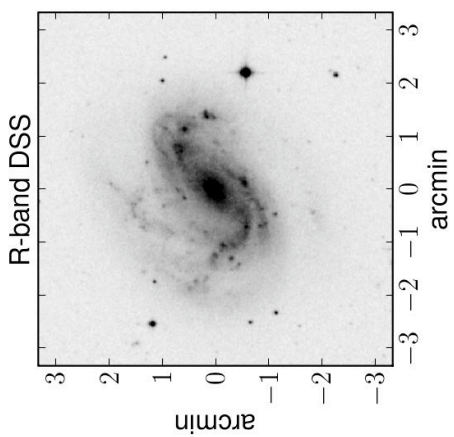

舫产

क ธิ

正

离 동

के

윰

पँ

क Q

ฮี

藏

है क्ट

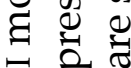

正采

ن च त्

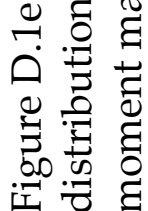




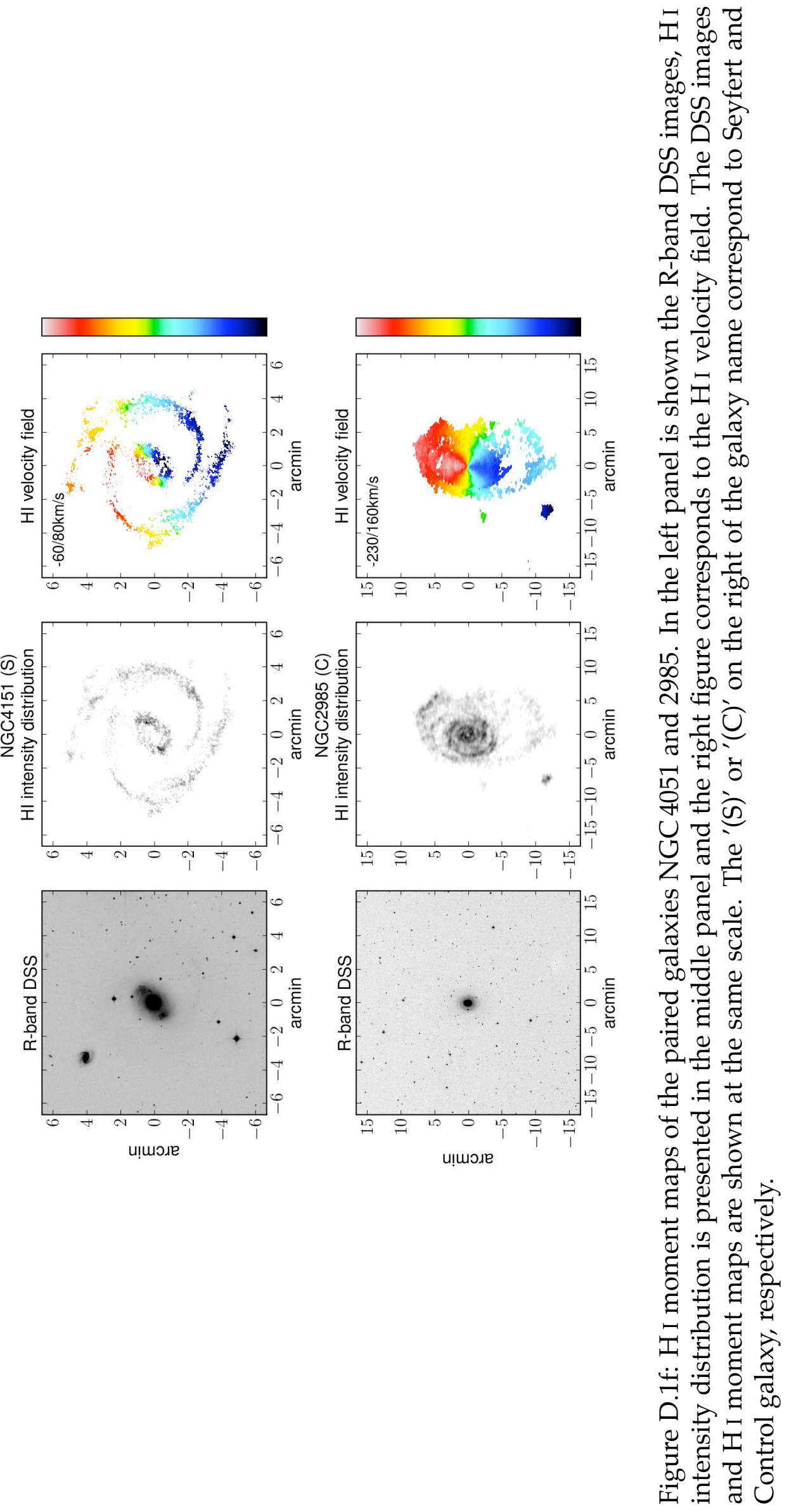




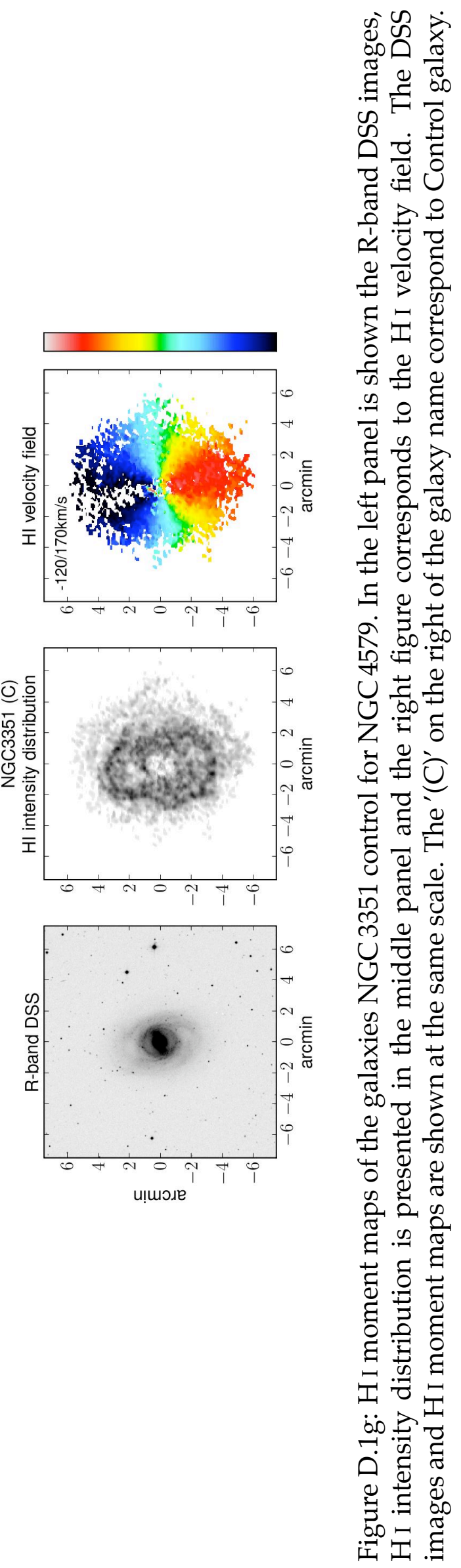




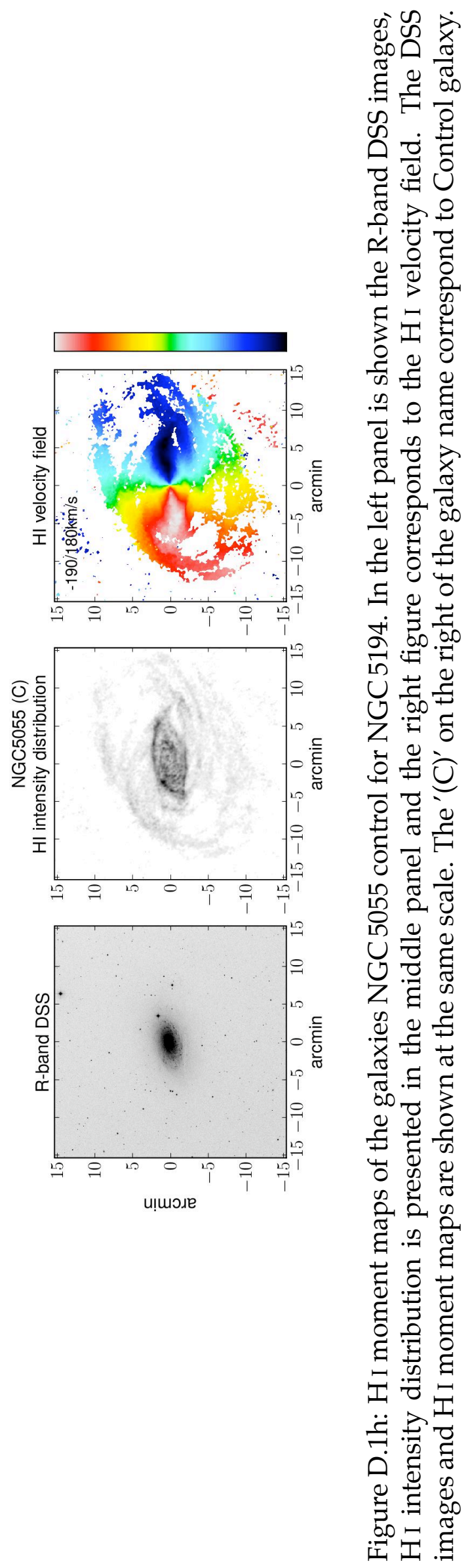




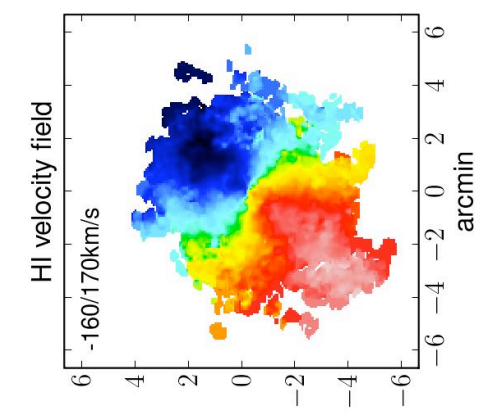

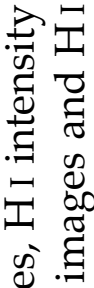

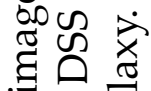
ஸू क

ฮृ ठํ

충ㅇ

舍 $\frac{0}{0}$

$5>$ के

号壳

के 0

. 58

옹

สำ

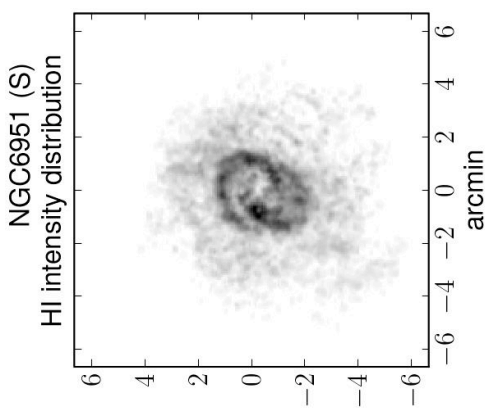

흐응

(c) 20

吾

투

두

จิ 600

$\bigcup$ 过

乙)

希吉

일

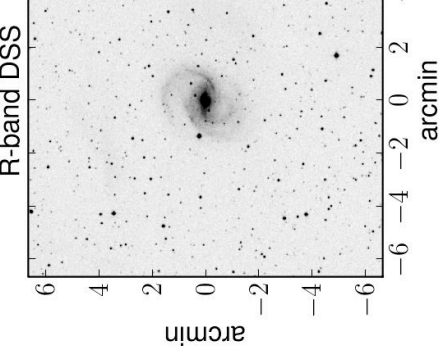

更

可 $\frac{0}{\pi}$

फे

ษ

प्छ क

क ฮै

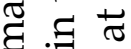

苹

है क

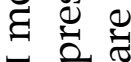

王.

$\therefore$ 즈

글 है

풀

氝 气

車䑻 


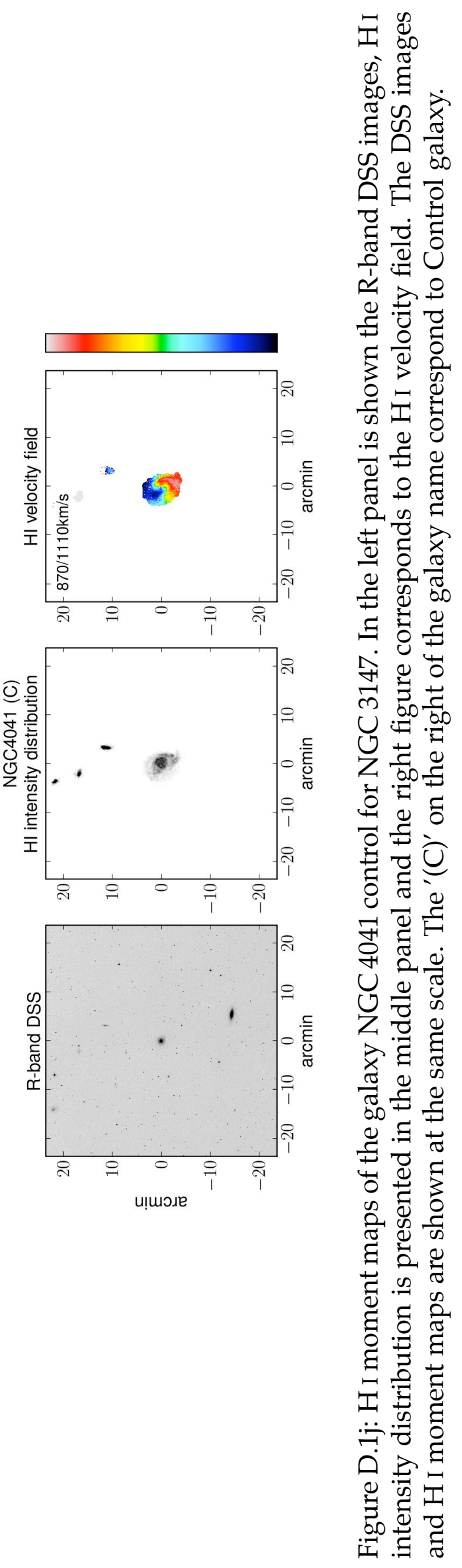




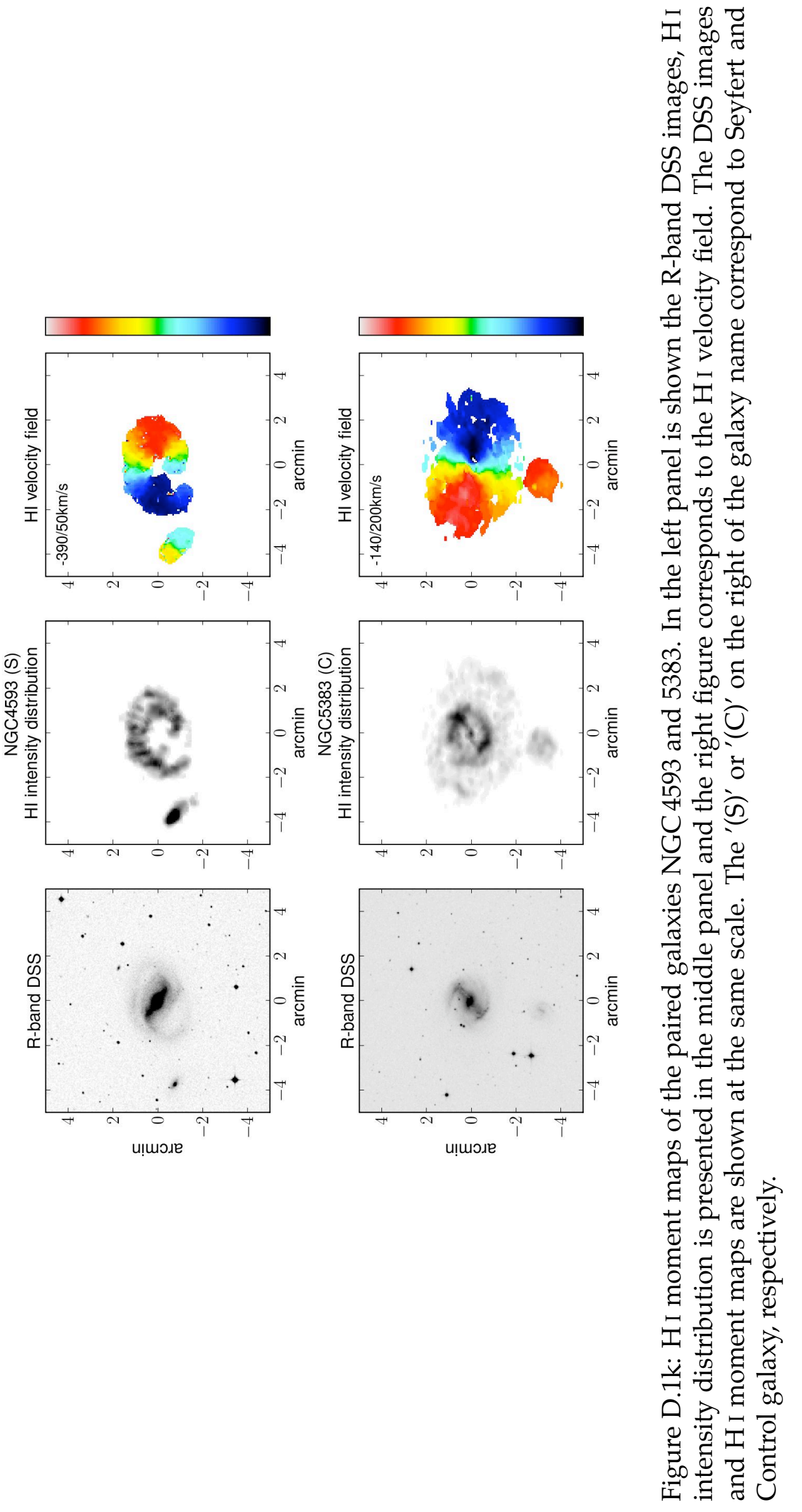




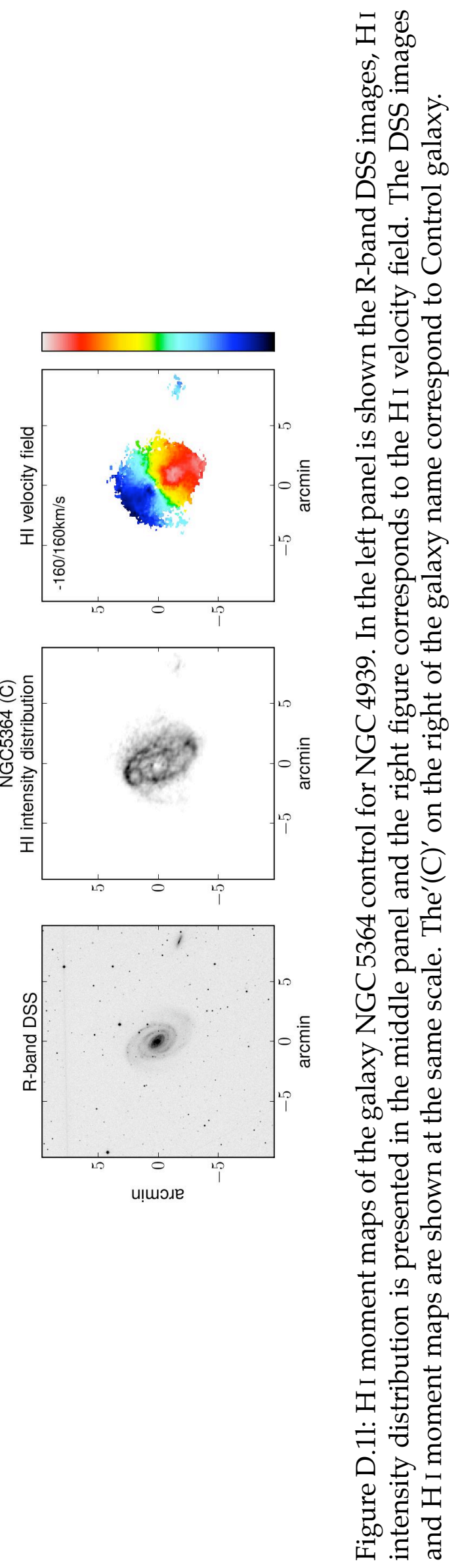




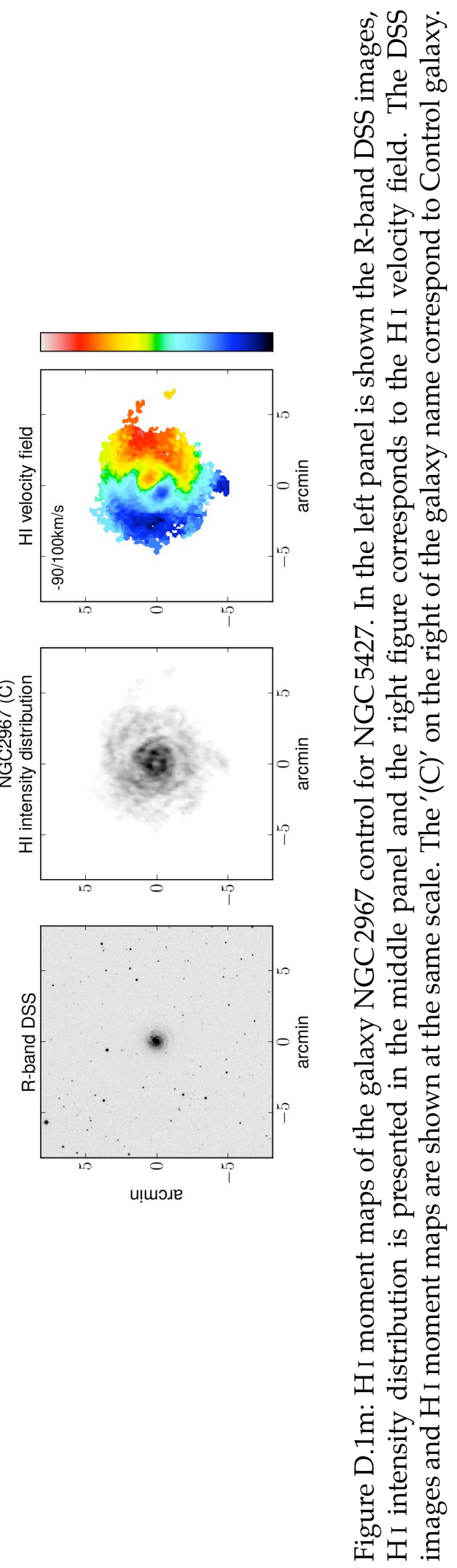




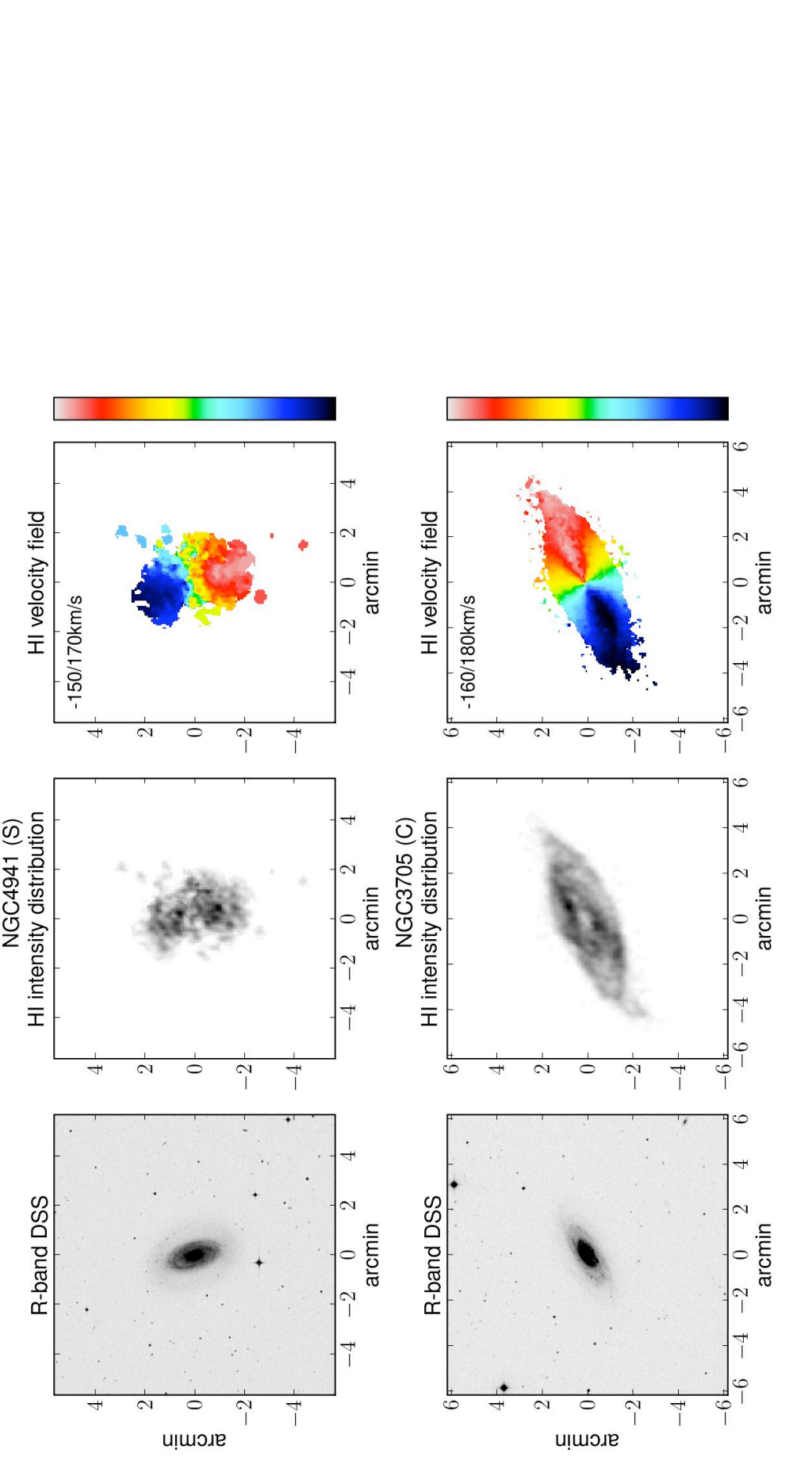

I

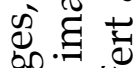

छึ की

की 우

正

ฮ ซ

तथ

릉

○

ᄃ

:

का

. $\frac{\mathscr{I}}{\pi}$

ฮ)

ㄷํㅇ

ॠ

巳

드 ᄋ

ம்

क 500

元艺论

अ

守吉

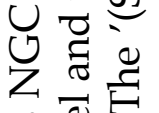

总

ฮิ

రृ

$\exists \approx$

ํㅗㄹ

Ð

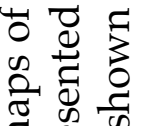

है के क

艺远.

घ.

든

छ .

ज़

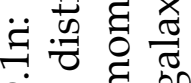

$0 \geqslant$

氙可

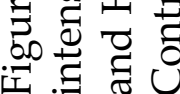




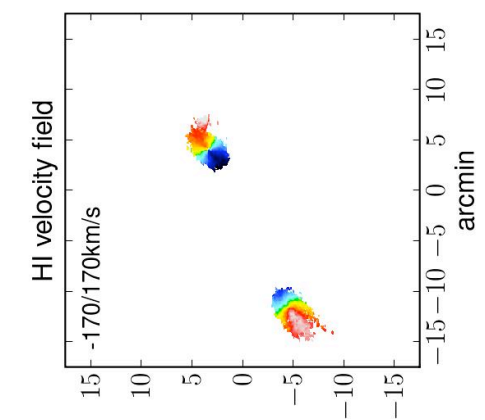

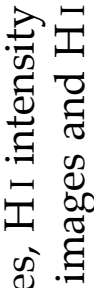

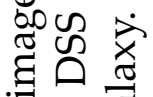
D赵

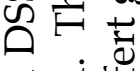

ฮ ह

○. क

농 우

Ð

$5>$ के

亖

के 8

. $I 8$

๘

สี

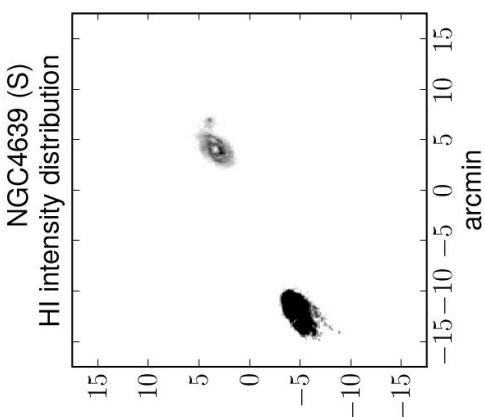

는 중

(c) 00

辛

$\Xi 0 \begin{gathered}0 \\ 0\end{gathered}$

ले Бే

요

U

Z

충

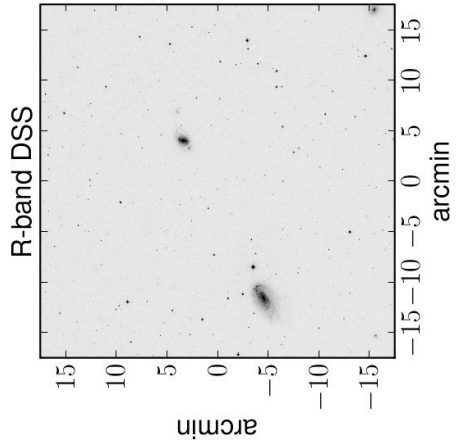

흐

क స छ

节

䒕 $\frac{0}{0}$

क ¿

휴 임

范 छ ฮ

คิ ขِ

ฮี

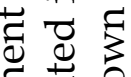

है 苟

छ

ప 兀ี

I $\infty$

$\ddot{0}$ ह

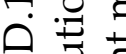

ว

艺

車要 


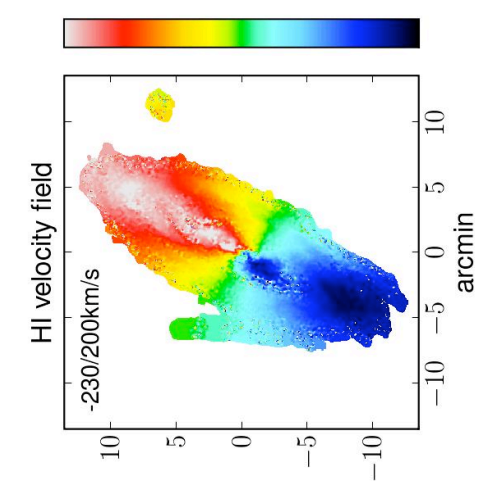

点工

过

$\exists$ क

T

क.

ఏ心

की

0.

ฮृ बे

1 $>0$

光.

I $\frac{0}{0}$

$5 \geq$ के

도 훙

क 号

Ð

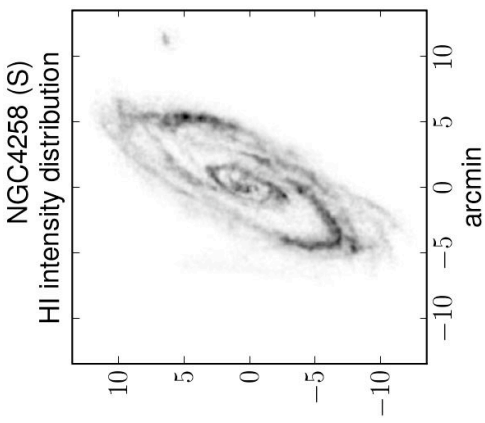

흥

๘

平氙

Ð 0 艺

$\infty$

ำ

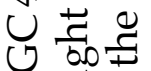

乙 I

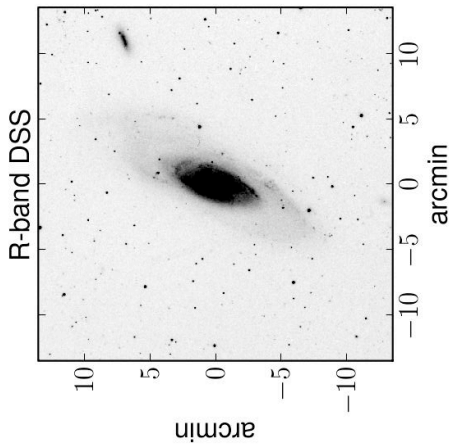

爻吉离

कृ

可

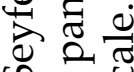

क ¿

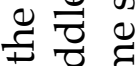

पै छ ส

مै ڤ)

$\Xi \Xi$

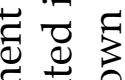

है क्षै

छ

Еี

‥ 2

ڤ. స

물

ว

$\exists$ ह

完告 


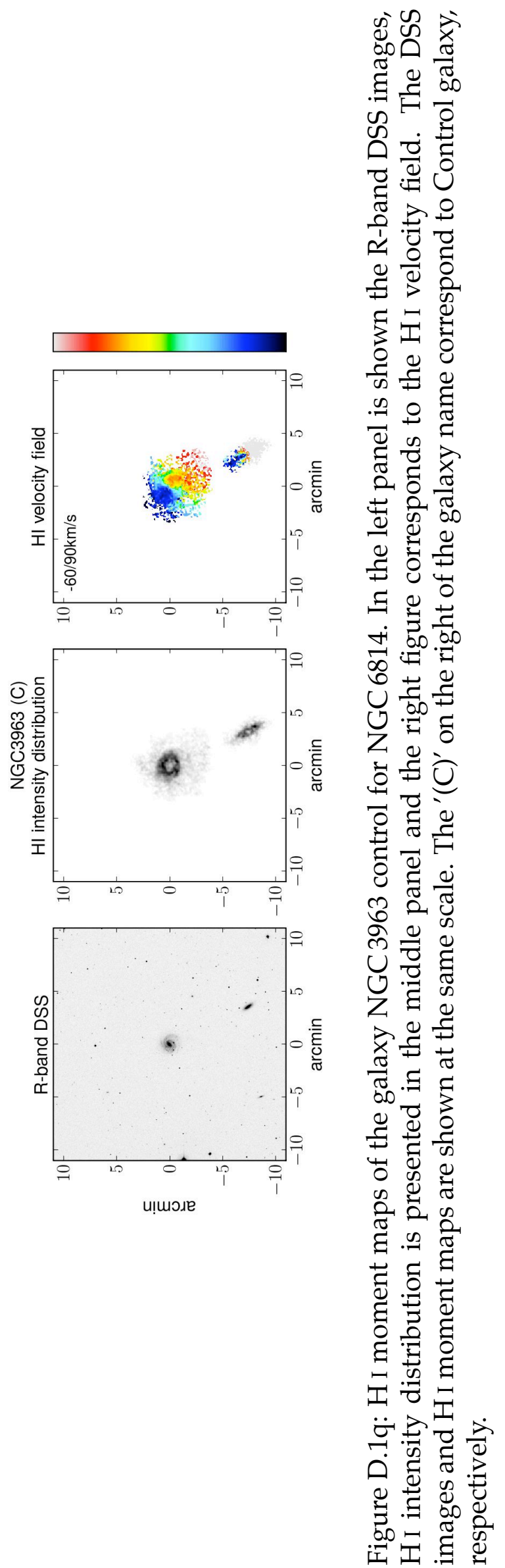




\section{Appendix E}

\section{Notes on individual galaxies.}

This chapter presents a description of the galaxies of our sample for which radio and/or optical data have been presented in this thesis. The galaxies are ordered following the order of the figures of Appendices C and D. Therefore the first nine sections present the galaxies of the VHIKINGS and SAURON/Seyfert projects, while the remaining sections concern only the galaxies from the VHIKINGS survey.

\section{E.1 NGC 1068/NGC 4548 pair}

\section{E.1.1 NGC 1068 (M 77)}

NGC 1068 is one of the most nearby Seyfert 2 galaxies $(D=14.4 \mathrm{Mpc})$, and it has been well studied at different wavelengths. Ho et al. (1997) classified this active galaxy as a Seyfert of type 1.8, but a dusty torus hides a Seyfert 1 nucleus (Antonucci \& Miller, 1985; Jourdain et al., 1994). NGC 1068's nucleus hosts a radio jet and an ionised cone at PA $\sim 10^{\circ}$ (Muxlow et al., 1996). Schinnerer et al. (2000b) summarised the different observed morphological structures : the outer disk and the outer oval, the latter being interpreted as a primary bar, a HI ring at its Outer Lindblad Resonance and the two-arms inner spiral at its Inner Lindblad Resonance. A secondary Near-Infrared (NIR) bar extends up to about 16 ". The SAURON data we presented here have been already published by Emsellem et al. (2006), and we refer the reader to this paper for a detailed analysis. We here only provide a brief description of the revealed structures.

The stellar continuum map (Fig. C.1a) shows elliptical isophotes, elongated along the PA of the NIR bar. The stellar velocity field (Fig. C.1a) shows strong departures from axisymmetry, with an S-shaped zero velocity line, and a slightly varying orientation of the kinematics major-axis. The velocity dispersion rises towards the centre, reaching $200 \mathrm{~km} \mathrm{~s}^{-1}$ at $\mathrm{R} \sim 10^{\prime \prime}$ and presents a drop in the inner $5^{\prime \prime}$ with values down to about $100 \mathrm{~km} \mathrm{~s}^{-1}$. The $h_{3}$ (Fig. C.2a) 
component shows a structure elongated along the PA of the NIR bar. The sign of $h_{3}$ is changing from the south-east (positive values) to the north-west (negative values). The $h_{4}$ (Fig. C.2a) map presents a ring-like structure of positive values between 8 and $12^{\prime \prime}$. Inside this ring, $h_{4}$ values decrease and become slightly negative. Gerssen et al. (2006) observed this galaxy with the Gemini Multi-Object Spectrograph (GMOS) IFU, covering the central $10 \times 8$ arcsec. Our stellar kinematics properties are in good agreement with theirs although they found a kinematic PA offset by about $13^{\circ}$ from ours, their FOV being too small to detect the change of orientation of the kinematic major-axis.

$\mathrm{H} \beta$ and [O III] distributions are quite different (Fig. C.1a). $\mathrm{H} \beta$ emission is very high in the inner $5^{\prime \prime}$, and traces the spiral arms outside this region. As for $\mathrm{H} \beta$, [O III] emission peaks in the central parts, with the distribution of [O III] becoming very asymmetric outwards. It is found predominantly in the north-east side, tracing the northern ionisation cone. Despite the significant differences between the distribution of the $\mathrm{H} \beta$ and [O III] line emission, their velocity fields and velocity dispersion maps are very similar (Figs. C.1a and C.3a). The velocity fields of $\mathrm{H} \beta$ and [O III] both display a prominent S-shaped zero velocity curve, evidence for strong deviations from circular motions. The velocity dispersion maps of $\mathrm{H} \beta$ and [O III] show a peak in the central $5^{\prime \prime}$ parts and then reach lower values outside. As expected, the $[\mathrm{O} \mathrm{III}] / \mathrm{H} \beta$ is high in the region of ionisation cone $(\sim 10)$, and lower in the spiral arms ([O III] $/ \mathrm{H} \beta<1)$, the latter being dominated by star formation regions (Figs. C.1a).

\section{E.1.2 NGC 4548 (M91)}

NGC 4548 is a non-active spiral galaxy classified as SBb, in the Virgo cluster. It hosts a large-scale stellar bar of $\mathrm{PA} \sim 80^{\circ}$ while the outer disk PA is around $-30^{\circ}$.

The HI distribution is elongated along the optical photometric PA and traces the outer spiral arms (Fig. D.1d, first row). It presents a depression in the central regions. North and west of the H I disk, two parallel lanes are observed elongated along the north-west direction. These features are not seen in previous H I observations (Vollmer et al., 1999). However, they conclude that their results are consistent with ongoing ram pressure stripping on the neutral gas disk. The off-disk features observed in our radio maps may correspond to gas removed via this scenario.

The H I kinematics displays regular rotation patterns. The kinematic PA is constant over the FOV at $135^{\circ}$ in agreement with previous studies (Guhathakurta et al., 1988; Vollmer et al., 1999). 


\section{E.2 NGC 3227/NGC 3169 pair}

\section{E.2.1 NGC 3227}

NGC 3227 is a well studied barred galaxy, interacting with the elliptical galaxy NGC 3326, and with an associated dwarf galaxy (Mundell et al., 2004). The galactic disc has an inclination of $56^{\circ}$, with an outer photometric major-axis at a position angle $P A=158^{\circ}$ (Mundell et al., 1995a), coincident with the majoraxis of the stellar bar. It is hosting a type 1.5 Seyfert nucleus (Ho et al., 1997).

Our HI moment maps are presented in the first row of Fig. D.1b. No H I emission is associated with the companion NGC 3226 and the southern plum is much more elongated and narrower than the plum on the north pointing towards NGC 3226. These two features are associated with the interaction between these two galaxies (Mundell et al., 1995b)

The central region has been mapped in ${ }^{12} \mathrm{CO}(1-0)$ and ${ }^{12} \mathrm{CO}(2-1)$ by Schinnerer et al. (2000a), who detect molecular gas very close to the nucleus $(\sim 13$ pc). The inner kpc regions host several complex features: a radio jet (PA $\sim-10^{\circ}$; Mundell et al. (1995b)), a conical NLR outflow at a PA of about $15^{\circ}$ (Mundell et al., 1995a), an $\mathrm{H} \alpha$ outflow at PA $\sim 50^{\circ}$ (Arribas \& Mediavilla, 1994), and a molecular nuclear ring (Schinnerer et al., 2000a).

Our SAURON stellar continuum map (Fig. C.1a) presents an elongated structure $\left(\mathrm{PA}=153^{\circ}\right)$, aligned with the stellar bar. The stellar velocity field is regular (Fig. C.1a), the major-axis being parallel to the galactic disc orientation. Outside $r>5^{\prime \prime}$, the stars rotate rigidly (isovelocity contours parallel to each others). The stellar velocity dispersion rises towards the centre, reaching $200 \mathrm{~km} \mathrm{~s}^{-1}$ in the inner $5^{\prime \prime}$. The $h_{3}$ and $h_{4}$ maps are featureless (Fig. C.2a).

Fig. C.1a presents the [O III] and the narrow component of $\mathrm{H} \beta$ emission lines. The central broad component for the $\mathrm{H} \beta$ line corresponding to nuclear emission (BLR) has been removed. [O III] distribution is rather asymmetric, elongated on the north side of the field and $\mathrm{H} \beta$ emission lines extends from south-east to east. The [O III] and $\mathrm{H} \beta$ velocity fields are similar: they show strong deviations from axisymmetry with a PA of the major-axis changing from $\sim 30^{\circ}$ in the central regions, to about $170^{\circ}$, close to the stellar kinematic major-axis orientation. The velocity dispersion of both $\mathrm{H} \beta$ and [O III] rise towards the nucleus, reaching $310 \mathrm{~km} \mathrm{~s}^{-1}$ and $360 \mathrm{~km} \mathrm{~s}^{-1}$, respectively in the centre. Finally, the $[\mathrm{O} \mathrm{III}] / \mathrm{H} \beta$ lines ratio map shows an elongated structure of high values $(>7)$ along the global kinematic major-axis (north-south).

The atomic gas in the outer disk $(\mathrm{R}>\sim 1.5 \mathrm{kpc})$ and the ionised gas and stars in the circumnuclear regions $(\mathrm{R}<\sim 1.5 \mathrm{kpc})$ present globally the same kinematic orientation, as seen in fig 5.21a. The kinematic PAs of the H I component in the outer disk (beyond $2.5 \mathrm{kpc}$ ) and of the stellar component in the inner regions are in very good agreement, at a constant value of about $160^{\circ}$ at all observed spatial scales. In the central regions, the kinematic PA of the ionised gas re- 
mains roughly constant for radii larger than $10^{\prime \prime}$, but with an offset of about $20^{\circ}$ with respect to the stellar and $\mathrm{H}$ I PA.

Since this galaxy presents evidence of strong interaction with a companion, this interaction and the nuclear activity are likely to be linked.

\section{E.2.2 NGC 3169}

NGC 3169 is a LINER galaxy, classified as Sa. It is interacting with NGC 3166 lying at about $8^{\prime}$ west of NGC 3169. Another smaller galaxy, NGC 3165 is situated $\sim 12^{\prime}$ south west of NGC 3169. The H I moment maps (Fig. D.1b, fourth row) display complex distribution and kinematics. No H I emission from the companion NGC 3166 is detected, but large spiral-like features arise from the disk of NGC 3169 towards NGC 3166, certainly due to tidal interaction. Our H I maps are in good agreement with the lower resolution data of Haynes (1981). In our maps, H I emission is detected, clearly associated with the second companion (NGC 3165) of NGC 3169. Haynes (1981) observed this component but could not disentangle it from the emission of NGC 3169 and 3166.

\section{E.3 NGC 2655/NGC 4459 pair}

\section{E.3.1 NGC 2655}

NGC 2655 is an early-type spiral galaxy, hosting a Seyfert 2 nucleus. Optical and radio spectroscopic data exhibit off-plane gas in the central part (Erwin \& Sparke, 2003), and a dusty polar ring seen in $\mathrm{H} \alpha$ (Keel \& Hummel, 1988). This galaxy is interacting with its companion NGC 2715 and presents remnant signatures of a past merger (Huchtmeier \& Richter, 1982). Our H I moment maps (Fig. D.1c, first row) show the companion at the east and the west tidal loop as shown by Huchtmeier \& Richter (1982). The H I velocity field presents a change of orientation in the central arcmin, from PA $\sim-90^{\circ}$ in the outer disk, aligned with the LON of the galaxies and the stellar circumnuclear kinematics PA (see Table. 5.1), to PA $\approx-180^{\circ}$ in the innermost region.

The stellar continuum map (Fig. C.1b) shows an elongated feature, along the east-west direction, which corresponds well with the PA of the line of maximum stellar velocity. The stellar velocity field (Fig. C.1b) is remarkably regular, with a PA around $-90^{\circ}$. The velocity dispersion map shows a drop in the inner $3^{\prime \prime}$. The $h_{3}$ and $h_{4}$ maps show no specific structures (Fig. C.2a).

$\mathrm{H} \beta$ and [O III] emission lines distribution and kinematics are similar (Figs. C.1b and C.3a). The emission lines are very bright in the inner 5". Away from the centre, the distribution maps show a bright knot to the south-east side, $15^{\prime \prime}$ away from the nucleus, and a lane at $10^{\prime \prime}$ on the west side: both features have been reported by Keel \& Hummel (1988). The SE knot corresponds 
to high $[\mathrm{O} I I I] / \mathrm{H} \beta$ ratio values $(\sim 4)$ similar to the ones observed in the nuclear region. This feature could be driven by the radio jet (Keel \& Hummel, 1988). The emission lines ratio is smaller in the west lane (around 2-3). The emission lines velocity fields show strong departures from axisymmetry, with an S-shaped zero velocity curve, the kinematic major-axis having a changing $\mathrm{PA}$, from $\sim 90^{\circ}$ in the inner $5^{\prime \prime}$ to near $180^{\circ}$ in the outer parts. As hinted by Sil'chenko \& Afanasiev (2004), the ionised gas rotates together with the stars in the inner $5^{\prime \prime}$, but follows the dust polar ring at radii $>5^{\prime \prime}-6^{\prime \prime}$, rotating perpendicularly to the galactic plane. [O III] and $\mathrm{H} \beta$ velocity dispersion maps show the same features: a rise in the inner $5^{\prime \prime}\left(\sim 220 \mathrm{~km} \mathrm{~s}^{-1}\right)$ and in the western feature $\left(305 \mathrm{~km} \mathrm{~s}^{-1}\right)$, and a roughly constant value outside (between 100 and $\left.150 \mathrm{~km} \mathrm{~s}^{-1}\right)$.

The kinematic PA of the atomic gas in the outer disk (beyond $\sim 2^{\prime} \approx 14 \mathrm{kpc}$ ) and the one of the stellar component in the circumnuclear regions are in good agreement, as seen in fig. 5.21a, while the ionised gas rotates almost perpendicularly to the stars in the central regions (Fig. 5.3) with a PA corresponding well with the orientation of the $\mathrm{HI}$ kinematic between 3.5 and $11 \mathrm{kpc}$. The H I rotation axis changes from a value similar to the PA of the kinematic major axis of the stars (in the central regions), which can be associated with the line of nodes of the galaxy (see Sec. 5.1.1), to a value perpendicular to the line of nodes and aligned with the ionised kinematic orientation, in the circumnuclear regions $(\mathrm{R}<2.5 \mathrm{kpc})$.

These kinematic features can be explained by a past encounter with the companion NGC 2715. Hence, the tidal interaction between NGC 2655 and its companion may have triggered large gas inflows towards the central parts of NGC 2655. In the case of this galaxy, the encounter with a nearby companion is likely to be the cause of the nuclear activity.

\section{E.3.2 NGC 4459}

This S0 galaxy is in the Virgo cluster. It is the control non-active galaxy for NGC 2655. The SAURON data presented here (Fig. C.1b) are part of the SAURON project (de Zeeuw et al., 2002) and were therefore already published elsewhere (Emsellem et al., 2004; Sarzi et al., 2006).

The stellar component of NGC 4459 shows roundish isophotes, and its stellar velocity field presents a very regular rotation pattern the major-axis of which is parallel to the line-of-nodes $\left(\mathrm{PA} \sim 77^{\circ}\right)$. The velocity dispersion rises towards the centre, reaching $200 \mathrm{~km} \mathrm{~s}^{-1}$ (Fig. C.1b). The $h_{3}$ and $h_{4}$ coefficients maps are presented in Fig. C.2a. $h_{3}$ is anticorrelated with the stellar velocity $V$. The $h_{4}$ map is rather featureless.

There is almost no ionised gas emission (our maps have been clipped at $3 \sigma$ ), except for the very centre $\left(R<3^{\prime \prime}\right)$.The emission lines velocity field seems to be dominated by rotation, and the velocity dispersion rises from about $80 \mathrm{~km} \mathrm{~s}^{-1}$ 
for $R>3^{\prime \prime}$ to $160 \mathrm{~km} \mathrm{~s}^{-1}$ for $\mathrm{H} \beta\left(200 \mathrm{~km} \mathrm{~s}^{-1}\right.$ for [O III]) inside $3^{\prime \prime}$. The [O III] $/ \mathrm{H} \beta$ line ratio rises regularly towards the centre.

\section{E.4 NGC 3627/NGC5806 pair}

\section{E.4.1 NGC 3627 (M 66)}

NGC 3627 is an SAB spiral galaxy hosting a Seyfert 2 nucleus. It is part of the Leo Triplet system, interacting with NGC 3623 and with evidence for a past interaction with NGC 3628 (Zhang et al., 1993). Previous radio and optical observations show asymmetric structures in the velocity field, non-circular gaseous motions and a molecular CO inner ring (Chemin et al., 2003). More recently, IFS data obtained by Afanasiev \& Sil'chenko (2005) showed evidence for strong gaseous radial motions.

The SAURON stellar continuum map shows an elongated structure along the photometric PA $\sim 170^{\circ}$ (Fig. C.1c). The velocity field presents a regular rotation pattern, with a slight twist of the zero-velocity line in the very central parts $\left(r<3^{\prime \prime}\right)$. The stellar rotation follows the orientation of the photometric majoraxis. The stellar velocity dispersion rises towards the central regions up to $120 \mathrm{~km} \mathrm{~s}^{-1}$. Finally, $h_{3}$ map shows a structure in the inner $5^{\prime \prime}$ anticorrelated with the stellar velocity, and $h_{4}$ rises towards the centre (Fig. C.2a).

The [O III] emission is concentrated in the inner $5^{\prime \prime}$, with some emission in an elongated structure, along the photometric PA (Fig. C.1c). As for [O III], $\mathrm{H} \beta$ emission is preponderantly observed in the central parts, with an additional bright spot about $15^{\prime \prime}$ south of the nucleus. The [O III] and $\mathrm{H} \beta$ kinematics are quite similar (Figs. C.1c and C.3a). Their velocity fields show strong deviations from axisymmetry: the orientation of the kinematic major-axis changes from the external regions where it is aligned with the stellar kinematics, toward the nucleus where the ionised gas kinematic major-axis deviates by $\sim 40^{\circ}$ from the stellar one. Afanasiev \& Sil'chenko (2005) suggested that strong radial motions may exist in the central parts of this galaxy. Both [O III] and $\mathrm{H} \beta$ velocity dispersion maps are almost featureless, with a slight depression in the very centre. Finally, the $[\mathrm{O} I I I]] / \mathrm{H} \beta$ lines ratio reaches its highest values in the inner $2^{\prime \prime}$.

\section{E.4.2 NGC 5806}

This non-active galaxy is the control for NGC 3627. Carollo et al. (2002) observed this galaxy with the HST / NICMOS2 camera in the near-infrared. They detect a prominent bulge and a stellar bar structure in the circumnuclear regions. They classified NGC 5806 as a galaxy with a concentrated nuclear star formation mixed with dust. 
The H I distribution traces well the optical disk (Fig. D.1d, fourth row) and is fairly asymmetric: weaker H I emission is detected in a south elongated structure. A central depression is observed. A companion is detected at about $140 \mathrm{kpc}$ South-East of NGC 5806 (this companion is not seen in the Fig. D.1d since it is a zoom of the central $\sim 17 \mathrm{kpc}$ ). The $\mathrm{H}$ I elongation in the south may be evidence of a weak tidal interaction with this companion. The H I kinematics is very regular, consistent with global rotation aligned with the outer disk major-axis.

Our SAURON data is presented in Figs. C.1c, C.3b and C.2b. The stellar morphology and kinematics are quite regular. The stellar continuum within the SAURON field of view is elongated along the outer photometric major-axis of the galaxy $\left(\mathrm{PA}=170^{\circ}\right)$. The stellar velocity field shows a regular rotation pattern with the kinematic-major axis aligned with the photometric major-axis. The velocity dispersion is rising toward the nucleus reaching about $120 \mathrm{~km} \mathrm{~s}^{-1}$ in the centre. $h_{3}$ presents a symmetric structure aligned and anticorrelated with the stellar velocities. $h_{4}$ is slightly rising towards the centre.

The $[\mathrm{O}$ III] and $\mathrm{H} \beta$ emission line distribution show elongated features along the photometric PA. That feature exhibits a ring-like structure in $\mathrm{H} \beta$, broken in the north-east part, at a radius $\sim 3^{\prime \prime}$. The $[\mathrm{O} \mathrm{III}] / \mathrm{H} \beta$ ratio shows rather low values in the $\mathrm{H} \beta$ ring $(\sim 0.3)$, consistent with star formation. The gas velocity maps are dominated by rotation, and resemble the stellar velocity field, though with a higher amplitude. The ionised gas velocity dispersion maps show no particular structures.

The kinematic of the central regions (stars) and of the outer disk ( $\mathrm{HI})$ present regular rotation patterns, with the same orientation. The radial values of the kinematic PAs of the stars and those of the atomic gas are in very good agreement, especially in the regions of overlap of the two observations, at a radius of about $4 \mathrm{kpc}$ (see Fig. 5.21b). The velocity curves of the atomic and ionised gas present a good continuation between the central regions $(R<4 \mathrm{kpc})$ and the outer disk $(\mathrm{R}>4 \mathrm{kpc})$.

\section{E.5 NGC 4051/NGC5248 pair}

\section{E.5.1 NGC 4051}

NGC 4051 is an SAB galaxy classified as S1.2 by Ho et al. (1997). The stellar bar extends up to $\sim 50^{\prime \prime}$ along a $\mathrm{PA} \approx 135^{\circ}$. Radio-observations show a triple source at a PA $\sim 73^{\circ}$ (Christopoulou et al., 1997), and the [O III] emission line distribution in the inner $3^{\prime \prime}$ is aligned with this radio component. The narrow-line profiles of NGC 4051 present strong blue wings. This blue-shifted component is also detected with SAURON in the $\mathrm{H} \beta$ and [O III] lines, and has therefore been fitted (see Sect. 2). Christopoulou et al. (1997) found evidence for a conical outflow, at 1 .'5 from the nucleus. 
Our Hi zeroth moment map (Fig. D.1e, second row) displays a symmetric distribution: an outer ring-like feature traces the optical spiral arms and a depression in the central arcmin is observed. The H I velocity fields is very regular with constant major-axis orientation, at a PA of $\sim-45^{\circ}$, similar to the photometric outer disk PA. Our H I distribution and kinematics maps are in good agreement with the moment maps of Liszt \& Dickey (1995) obtained with the VLA in D-array configuration.

Our SAURON stellar continuum map (C.1d) shows regular isophotes elongated along the PA of the large-scale bar. The stellar velocity field presents a regular and symmetric rotation pattern, the rotation axis being aligned with the photometric major-axis $\left(\mathrm{PA} \sim 135^{\circ}\right.$ ). The stellar velocity dispersion decreases in the inner $5^{\prime \prime}$ down to $\sim 50 \mathrm{~km} \mathrm{~s}^{-1}$.

The emission line intensity is slightly extended towards the north-east, consistent with the ionised [O III] outflow cone observed by Christopoulou et al. (1997). The orientation of the ionised gas kinematic major-axis varies with radius: it is aligned with the stellar one in the outer part of the SAURON FOV, and then deviates by about $30^{\circ}$ from the line-of-nodes of the galaxy. The $\mathrm{H} \beta$ emission line velocity dispersion reaches its highest values in the inner $5^{\prime \prime}$, while a decrease of dispersion is observed in these central regions for [O III]. The [O III] / $\mathrm{H} \beta$ lines ratio peaks at about $3^{\prime \prime}$ north-east from the nucleus, within the outflow region (Christopoulou et al., 1997).

The kinematics of the stars in the central regions and of the atomic gas in the outer disk present similar regular rotation patterns. The kinematic PAs of these two components are in good agreement (see fig. 5.21a). No companion is detected in $\mathrm{HI}$ at a radial distance of about $6^{\prime}$ (which corresponds to $\sim 30$ $\mathrm{kpc}$ ) and the $\mathrm{H}$ I morphology maps present no sign of past merger, hence the nuclear activity of NGC 4151 does not seem have been triggered by an external mechanisms. Dynamical perturbations due to secular processes, such as stellar bars and spiral arms, seem to be a more sensible hypothesis to explain the nuclear activity of this galaxy.

\section{E.5.2 NGC 5248}

NGC 5248 is a nearby inactive spiral galaxy, control for both NGC 4051 and NGC 6951. It harbours two prominent circumnuclear rings (Laine et al., 2001) and an inner molecular spiral within $1.5 \mathrm{kpc}$ driven by the large scale bar (Jogee et al., 2002b). The arms of the spiral connect with the inner circumnuclear ring. Jogee et al. (2002b) also mention the presence of streaming motions in this galaxy.

The stellar continuum shows an elongated structure along the global disk orientation at $P A \sim 150^{\circ}$ (Fig. C.1d). The velocity field presents a regular rotation pattern, the major-axis of which is oriented at a PA of $115^{\circ}$, aligned with the photometric major-axis ( $P A=105^{\circ}$, Jogee et al., 2002b). A $\sigma$-drop is re- 
vealed by our velocity dispersion map in the inner $3^{\prime \prime}$ with an amplitude of $\sim 60 \mathrm{~km} \mathrm{~s}^{-1}$. The $h_{3}$ and $h_{4}$ coefficients are shown in Fig. C.2b. $h_{3}$ is anticorrelated with the stellar velocity. $h_{4}$ is rising toward the centre, and presents an elongated structure following the stellar continuum.

The $\mathrm{H} \beta$ emission line distribution shows a ring-like structure with a radius of $\sim 5^{\prime \prime}$. The [O III] distribution is concentrated within the central few arcseconds, and in the two brightest peaks of the ring. This ring-like structure corresponds to the known ring of HII region described by Laine et al. (2001). The $[\mathrm{O} I I I] / \mathrm{H} \beta$ emission line ratio is lower inside the ring (0.15), consistently with the presence of star formation, and rises slightly towards the very centre (0.7). The ionised gas velocity field is dominated by rotation with its major-axis aligned with the stellar kinematic one. The $\mathrm{H} \beta$ velocity dispersion map shows a depression in the star formation ring $\left(50 \mathrm{~km} \mathrm{~s}^{-1}\right)$, and slightly rises towards the very centre $\left(80 \mathrm{~km} \mathrm{~s}^{-1}\right.$, Fig. C.3b). The [O III] velocity dispersion map shows not specific features, with roughly constant values around $50 \mathrm{~km} \mathrm{~s}^{-1}$.

\section{E.6 NGC 4151/NGC 2985 pair}

\section{E.6.1 NGC 4151}

NGC 4151 is one of the most well-studied galaxies of our sample. For a review on this galaxy and the AGN properties, see Ulrich (2000). It is an almost faceon barred spiral galaxy hosting a Seyfert type 1.5 active nucleus (Ho et al., 1997). The large-scale weak bar is elongated along a PA of about $130^{\circ}$ and the photometric major-axis orientation is close to $20^{\circ}$ (Mundell \& Shone, 1999). Its nuclear continuum and BLR emission are highly variable. It contains a radio jet along a PA of $77^{\circ}$ and its Narrow Line Region (NLR) is extending over $\sim 10^{\prime \prime}$, and is aligned with the jet (Mundell et al., 2003).

The H I archive data of NGC 4151 used in this thesis have been already published by Mundell et al. (1999) and Mundell \& Shone (1999). The central H I distribution (Fig. D.1f, third row) shows a central ring-like structure which traces the large scale stellar bar. Then outer H I spiral arms are seen (Mundell et al., 1999; Mundell \& Shone, 1999). These spiral arms are detected in optical wavelengths but are very weak (Fig. 4.2.1). The H I kinematics presents regular patterns consistent with global rotation. Non-circular motions are observed, associated with the large-scale stellar bar as shown by Mundell \& Shone (1999).

Our stellar continuum map (Fig. C.1e) presents rather round isophotes. The stellar velocity field shows a twist of the zero-velocity line in the inner 5 " which corresponds to the region where the BLR dominates the spectral features. A $\sigma$-drop is observed in the central part of the velocity dispersion map, but could be due to the BLR contamination. The [O III] and $\mathrm{H} \beta$ emission lines distributions are elongated on the south-west direction at a PA of about $50^{\circ}$ 
associated with the NLR and the Extended NLR (ENLR, $R>5^{\prime \prime}$ Asif et al., $2005)$. The emission lines ratio reaches very high values $(\sim 12)$ in this region. The ionised gas kinematics is dominated by rotation, but with some disturbances on the north-west side. [O III] emission line velocity dispersions peak in the inner few arcsec $\left(\sim 280 \mathrm{~km} \mathrm{~s}^{-1}\right)$. Outside the very central parts, the velocity dispersion is between 50 and $130 \mathrm{~km} \mathrm{~s}^{-1}$. It increases at the edges of our map, corresponding to the location of a dusty ring (Vila-Vilaro et al., 1995). Asif et al. (1998) revealed the presence of streaming motions along this dust structure, which may be associated with the high [O III] velocity dispersion observed in our SAURON maps. $\mathrm{H} \beta$ velocity dispersion is flat in the outer parts $\left(R>10^{\prime \prime}\right.$, with $\sigma$ between 80 and $\left.130 \mathrm{~km} \mathrm{~s}^{-1}\right)$ and rises regularly towards the centre, reaching $200 \mathrm{~km} \mathrm{~s}^{-1}$ in the inner $3^{\prime \prime}$. The maximum velocity dispersion $\left(\sim 350 \mathrm{~km} \mathrm{~s}^{-1}\right)$ is reached at about 3"5 north-east of the centre.

No companion of NGC 4151 is detected at a maximum distance of about $50 \mathrm{kpc}$ with similar velocities and the H I distribution map shows no evidence of a past merger (see fig. D.1f). The nuclear activity of NGC 4151 seems then not due to external triggering. Despite some kinematic perturbations from the spiral arms and the bar, the $\mathrm{HI}$ and stellar velocity fields are in good agreement, with similar global orientations, as shown in fig. 5.21a. This figures shows as well that the velocity curves of the atomic gas in the outer disk $(\mathrm{R}>\sim 1.5 \mathrm{kpc})$ and of the ionised gas in the central regions $(\mathrm{R}<\sim 1.5 \mathrm{kpc})$ present similar values and shape in the overlap region of the radio and optical data, between 1.2 and $2 \mathrm{kpc}$.

In the case of NGC 4151, the origin of the nuclear activity is then to be sought in the dynamical structures of the galaxy, and in the perturbations due to e.g. the stellar bars of the spiral arms.

\section{E.6.2 NGC 2985}

NGC 2985 is an early-type spiral galaxy, with regular gas and stellar distributions. It is the control for NGC 4151. The disk orientation is determined by an inclination of $i=40^{\circ}$ and a major-axis position angle of $P A=180^{\circ}$. The H I distribution and kinematic of NGC 2985 is regular in the central $5^{\prime}$, then a large one-armed spiral-like feature is observed (Fig. D.1f, third row). This spiral arm is certainly due to the tidal interaction with the companion NGC 3027 as reported by Noordermeer et al. (2005). NGC 3027 lies at about $130 \mathrm{kpc}$ west of NGC 2985 and is not seen in the presented FOV of Fig. D.1f. In addition to this companion, a H I cloud is detected $\sim 12$ ' (corresponding to about $80 \mathrm{kpc}$ ) south east of NGC 2985.

The stellar velocity field of NGC 2985 shows a regular and symmetric pattern, consistent with motions in an axisymmetric gravitational potential (Fig. C.1e). The PA of the kinematic major-axis $\left(-2^{\circ}\right)$ is aligned with the photometric major-axis of the outer disc. The velocity dispersion rises inwards, with $100 \mathrm{~km} \mathrm{~s}^{-1}$ at $15^{\prime \prime}$ from the centre and $150 \mathrm{~km} \mathrm{~s}^{-1}$ in the inner $5^{\prime \prime}$. The $h_{3}$ 
map displays a change of sign from the south (positive values) to the north (negative values) (Fig. C.2b), following the kinematic major-axis. The $h_{4}$ map is featureless (Fig C.2b), with a slightly positive value everywhere in the FOV.

There is very little emission from the ionised gas $\mathrm{H} \beta$ and [O III] in NGC 2985 (Fig. C.1e). The emission lines flux peaks in the inner $2^{\prime \prime}$ with very little flux outside. The $\mathrm{H} \beta$ (Fig. C.3b) and [O III] (Fig. C.1e) kinematics are similar: the velocity fields are regular, showing the same overall symmetry as the stellar velocity field. The gaseous velocity dispersion maps show no specific features, with values rising towards the centre $\left(200 \mathrm{~km} \mathrm{~s}^{-1}\right.$ for [O III], $160 \mathrm{~km} \mathrm{~s}^{-1}$ for $\mathrm{H} \beta$ ). Finally, the $[\mathrm{O} \mathrm{III}] / \mathrm{H} \beta$ line ratio reaches values up to $1.5-2$ in the centre and decreases outwards (for $\mathrm{R}>10$ ").

Despite showing large radial variations, the stellar and H I kinematic PAs are in very good agreement, in particular in the overlap region of the radio and optical observations (at a radius of $\sim 2 \mathrm{kpc}$ ) as shown in fig. $5.21 \mathrm{~b}$. The velocity curves in the central parts (ionised gas) and in the outer disk present similar shapes and values around $2 \mathrm{kpc}$.

\section{E.7 NGC 4579/NGC 3351 pair}

\section{E.7.1 NGC 4579 (M 58)}

NGC 4579 is an active galaxy classified as S1.9/L1.9 (Ho et al., 1997). It hosts a NIR stellar bar of $9 \mathrm{kpc}$ of diameter, oriented along PA $=58^{\circ}$. García-Burillo et al. (2005) observed this galaxy with the Plateau de Bure Interferometer (CO) as part of the NUGA survey. They found a nuclear molecular spiral from $R \sim 1$ kpc down to $\sim 200$ pc, driven by the stellar bar and detected highly noncircular motions over the spiral arms. They interpreted these perturbations as outflow motions.

Fig. C.1f presents our SAURON maps for this galaxy. The stellar continuum isophotes are rather round in the centre and slightly elongated along a PA of $\sim 60^{\circ}$ corresponding to the orientation of the stellar bar. The stellar velocity field presents a very regular rotation pattern, with a kinematic major-axis oriented at a PA of $95^{\circ}$. The stellar velocity dispersion rises regularly towards the centre. The $h_{3}$ map exhibits a small structure within $5^{\prime \prime}$ anticorrelated with the stellar velocity. $h_{4}$ is rather constant except in the very centre $\left(r<2^{\prime \prime}\right)$ where it drops (Fig. C.2b).

[O III] and $\mathrm{H} \beta$ distributions and kinematics are very similar (Figs. C.1f and C.3b). The ionised gas intensity maps show a spiral-like structure which corresponds to the nuclear molecular structure observed by García-Burillo et al. (2005). The $[\mathrm{O} \mathrm{III}] / \mathrm{H} \beta$ ratio presents lower values in the spiral arms than outside. The ionised gas kinematics is complex and shows strong departures from axisymmetry. The gas kinematic major-axis $\left(P A=100^{\circ}\right)$ is almost perpendic- 
ular to the stellar bar, and varies within the SAURON field of view. Despite the similarities between $\mathrm{H} \beta$ and [O III] distributions and velocity fields, their velocity dispersions are significantly different. The $\sigma_{[O I I I]}$ map presents an elongated structure of high values along the stellar bar (Fig. C.1f), while $\sigma_{\mathrm{H} \beta}$ decreases in the central parts Fig. C.3b.

\section{E.7.2 NGC 3351 (M95)}

NGC 3351 is a barred inactive spiral galaxy. It is the control object for NGC 4579. NGC 3351 hosts a large-scale stellar bar and an inner molecular bar-like feature in the centre (Devereux et al., 1992). The latter molecular structure is aligned with the major-axis of the galaxy $\left(\mathrm{PA} \sim 10^{\circ}\right)$ and perpendicular to the large scale bar. Two rings of HII regions associated with resonances due to the stellar bar exist: one inner ring with a radius of $10^{\prime \prime}$ and another at $70^{\prime \prime}$ from the centre. Recent CO observations (Jogee et al., 2005) show evidence of non-circular streaming motions in the inner region.

Our H I moment maps reveal regular distribution and kinematics (Fig. D.1g, second row). H I emission is weaker in the central arcmin, and the H I velocity field is very regular, aligned with the outer disk PA. No companion is detected within a distance of $\sim 80 \mathrm{kpc}$.

The SAURON stellar continuum map (Fig. C.1f) shows a ring-like structure with $6^{\prime \prime} \lesssim R \lesssim 10^{\prime \prime}$, which corresponds well with the HII ring at the presumed ILR of the large scale bar. The stellar velocity field is regular throughout the field of view. Stellar velocity dispersions are slightly lower (by $\sim 20 \mathrm{~km} \mathrm{~s}^{-1}$ ) inside the ring than outside. $h_{3}$ values are anticorrelated with stellar velocities. $h_{4}$ rises regularly towards the centre (Fig. C.2b).

[O III] and $\mathrm{H} \beta$ distributions and kinematics do not differ much from each others. The intensity maps clearly show the known HII ring at $\sim 10^{\prime \prime}$ (Fig. C.1f), and there is very little emission outside that structure. The gas velocity maps are regular, following the orientation observed in the stellar velocity field. The [O III] velocity dispersion map is almost featureless, with only a slight gradient from north-east $\left(100 \mathrm{~km} \mathrm{~s}^{-1}\right)$ to south-west $\left(140 \mathrm{~km} \mathrm{~s}^{-1}\right)$. There is a velocity dispersion drop $\left(\sim 50 \mathrm{~km} \mathrm{~s}^{-1}\right)$ for $\mathrm{H} \beta$ in the ring (Fig. C.3b). The [O III] $/ \mathrm{H} \beta$ lines ratio map show low values in the inner regions $(\lesssim 0.1)$, as expected from star formation. [O III] $/ \mathrm{H} \beta$ ratio is significantly higher outside the ring $(\sim 1)$, probably mostly due to the uncertainty in the emission line flux there.

The kinematics of NGC 3351 are very regular and consistent with global rotation motions at all spatial scales. The stellar and atomic gas kinematic PAs present similar values around $-179^{\circ}$, and the velocity curves in the inner regions $(\mathrm{R}<\sim 800 \mathrm{pc})$ and in the outer disk $(\mathrm{R}>\sim 800 \mathrm{pc})$ are in good agreement in shape and values, as seen in the fig. 5.21b. 


\section{E.8 NGC5194/NGC5055 pair}

\section{E.8.1 NGC5194 (M 51)}

NGC 5194 is an almost face-on spiral galaxy in interaction with NGC 5195. Its nucleus is classified as a Seyfert type 2 (Ho et al., 1997). Many studies have been made on the structure and the nucleus of this nearby galaxy. A large outflow bubble extends up to $\sim 9^{\prime \prime}(\sim 42$ pc) north of the nucleus and a bright cloud is located about $3^{\prime \prime}$ south of the nucleus. These structures have been observed at different wavelengths (Ford et al., 1985), and they are found to trace the biconical NLR along a PA of $163^{\circ}$. NGC 5194 is hosting a radio-jet which interacts with the surrounding interstellar medium (Ford et al., 1985; Crane \& van der Hulst, 1992).

The SAURON stellar continuum map (Fig. C.1f) shows the presence of dust lanes. The stellar velocity field has a regular rotation pattern with its major-axis at a PA $\sim-165^{\circ}$, deviating by $\sim 25^{\circ}$ from the presumed line of nodes orientations ( $\mathrm{PA}=170^{\circ}$, Tully, 1974). The stellar velocity dispersion is featureless. $h_{3}$ is anticorrelated with the stellar velocities and the $h_{4}$ map shows no particular feature (Fig C.2b)

$\mathrm{H} \beta$ emission line flux is very high in the centre and for $R>5^{\prime \prime}$ it traces the spiral arms of the galaxy. The [O III] emission line flux traces the ionisation cone of NGC 5194. The [O III] intensity map shows the outflow bubble in the north and the bright cloud in the south of the nucleus corresponding to the end of the radio-jet. The emission line ratio [O III] $/ \mathrm{H} \beta$ peaks in the centre (with values up to 8) and in the southern cloud (values between 3 and 5). The ionised gas velocity field seems to be dominated by regular galactic rotation, but presents some distortions very probably due to the outflow observed in the inner $10^{\prime \prime}$. The ionised gas velocity dispersion rises towards the centre.

\section{E.8.2 NGC 5055 (M 63)}

NGC 5055 is a spiral non-active galaxy, control for NGC 5194. Previous radio and optical studies show an overall remarkable regularity and symmetry in its morphology and kinematics (Thornley \& Mundy, 1997; Battaglia et al., 2006). There are evidence for a tidal interaction with a companion (UGC 8313), and the outer disk of NGC5055 is warped. Blais-Ouellette et al. (2004) studied the $\mathrm{H} \alpha$ emission of NGC5055 with a Fabry-Perot: they found two velocity components in the inner $8^{\prime \prime}$ of this galaxy. One component is consistent with the global disk kinematics while the second one exhibits a pattern compatible with a bipolar outflow or a counter-rotating disk.

Our HI moments maps present regular distribution and kinematics (Fig. D.1h, first row). The H I intensity traces the optical disk and presents an outer large-scale ring-like or spiral-like pattern. These features have been 
previously reported by Battaglia et al. (2006). Although, slight wiggles of the zero-velocity line are observed in the $\mathrm{H}$ I velocity field map, the $\mathrm{H}$ I kinematics presents regular global rotation patterns.

Our SAURON stellar continuum map presents an asymmetry towards the north-west (Fig. C.1g). The stellar velocity field shows very regular rotation pattern with the kinematic major-axis aligned with the photometric one at a PA of $100^{\circ}$. The velocity dispersion rises in the very centre $\left(r<2^{\prime \prime}\right) . h_{3}$ is anticorrelated with the stellar velocities, and the $h_{4}$ map shows no particular feature (Fig C.2b).

The ionised gas $\mathrm{H} \beta$ and [O III] are mainly concentrated in the inner $5^{\prime \prime}$. The [O III] distribution shows a V-shaped structure towards the north, while the $\mathrm{H} \beta$ intensity map shows a number of hot spots broadly distributed over the FOV. The [O III] and $\mathrm{H} \beta$ velocity fields are very regular and similar to the stellar velocity field (except for their higher amplitudes). [O III] velocity dispersion maps is featureless but $\mathrm{H} \beta$ velocity dispersion map shows an elongated structure of high dispersion roughly aligned with the kinematic major-axis. Finally, the $[\mathrm{O}$ III] $/ \mathrm{H} \beta$ lines ratio reaches its highest values in the region of high [O III] emission line flux.

\section{E.9 NGC 6951/NGC5248 pair}

\section{E.9.1 NGC 6951}

NGC 6951 is a spiral galaxy which has a large stellar bar $\left(\mathrm{PA} \sim 90^{\circ}\right)$. Its nucleus is classified as Seyfert 2 (Ho et al., 1997), but Pérez et al. (2000) suggest that it could be considered as a transition object, between a very high excitation LINER and a Seyfert 2. The ionised gas $(\mathrm{H} \alpha)$ is mainly concentrated in the nuclear region and form an annulus of radius $\sim 5^{\prime \prime}$, in which massive star formation is taking place. Pérez et al. (2000) interpreted the $\mathrm{H} \alpha$ kinematics in the circumnuclear zone as a series of nested disks, decoupled from one another.

Fig. D.1i presents our H I moment maps for NGC 6951. The H I emission traces the optical features (stellar bar and spiral arms) and extends well beyond the optical disk. A H I depression is observed between the bar and the northern spiral arm. The Hi kinematics is consistent with global rotation, with a PA of about $140^{\circ}$, aligned with the outer ( $\mathrm{H}$ I and optical) disk PA. The zero-line velocity is twisted in the central regions, certainly due to the barred potential.

The stellar continuum presents isophotes elongated along a PA consistent with the galactic disk major-axis (PA $\sim 138^{\circ}$ Rozas et al., 2002). The stellar velocity field shows regular rotation pattern with the major-axis aligned with the line-of-nodes of the galaxy. The stellar velocity dispersion shows a drop in the inner $5^{\prime \prime} . h_{3}$ is anticorrelated with the stellar velocities, and $h_{4}$ rises regularly towards the centre (Fig. C.2b). 
The $\mathrm{H} \beta$ emission line distribution map shows a inner broken ring elongated along the major-axis of the galaxy $\left(P A=137^{\circ}\right)$ while [O III] is dominated by the AGN contribution, its intensity maps showing a compact central peak in the inner $3^{\prime \prime}$, with no particular feature outside. The emission line ratio [O III] / $\mathrm{H} \beta$ reaches very low values in the ring $(0.2)$, consistent with the presence of ongoing star formation in the annulus, and rises towards the centre (values of up to 6) where the effect of the AGN is prominent. The velocity fields are quite regular, dominated by the global rotation, and aligned with the stellar kinematics. The emission line velocity dispersion rises rapidly towards the centre reaching about $100 \mathrm{~km} \mathrm{~s}^{-1}$ close to the nucleus for both the $\mathrm{H} \beta$ and [O III] emission lines. These values are in good agreement with those found by Pérez et al. (2000).

NGC 6951 presents regular H I stellar and ionised gas kinematics, with kinematic PAs of these three components in very good agreement (see Fig. 5.21a). Moreover, the $\mathrm{H}$ I distribution map presents no evidence of recent tidal perturbation or merger (Fig. D.1i), and no companion is detected within about $120 \mathrm{kpc}$. Therefore the nuclear activity seems not to have been fuelled via external perturbations for this galaxy. The kinematic PAs radial variations from $\sim 3 \mathrm{kpc}$ to a few hundreds of parsecs can be explained by dynamical structures such as the stellar bar and the spiral arms. Such structures are to be investigated to constrain the different fuelling mechanisms in work in NGC 6951.

The control inactive galaxy of NGC 6951 is NGC 5248 described in the section E.5.2.

\section{E.10 NGC 3147/NGC 4041 pair}

\section{E.10.1 NGC 3147}

This SA spiral galaxy is hosting an active nucleus of type 2. The H I moment maps of this galaxy used in our study are presented and described in details by Haan et al. (2007).

\section{E.10.2 NGC 4041}

NGC 4041 is a non-active spiral galaxy,s classified as SAbc. It is hosting a small stellar bar, as revealed by K-band observation (Elmegreen et al., 1999). The H I distribution displays large outer spiral arms in the north and south (Fig. D.1j, first row). Three other galaxies are detected in the FOV. The H I velocity field presents a change of orientation with kinematic $\mathrm{PA} \sim-150^{\circ}$ in the outer regions and $\mathrm{PA} \sim-90^{\circ}$ in the inner $15^{\prime \prime}$. The H I kinematics in the central regions is similar to the molecular gas kinematics observed by Sakamoto et al. (1999). 


\section{E.11 NGC 4593/NGC5383 pair}

\section{E.11.1 NGC 4593}

NGC 4593 is a Seyfert 1 galaxy hosting a large-scale bar. NGC 4593 has two close companions: MGC-01-32-033 at about $4^{\prime}$ east and SVEN 314 at $2^{\prime}$ northwest. Radio continuum observations reveal faint radio lobes extending up to $26^{\prime \prime}(4.9 \mathrm{kpc})$ along a PA of $\sim 90^{\circ}$ (Gallimore et al., 2006).

Our H I zeroth and first moment maps are displayed in the second row of Fig. D.1k. NGC 4593 presents an outer ring of neutral hydrogen, broken in its southern part. This H I distribution traces the optical spiral arms. H I is also detected for the companion MGC-01-32-033 but not for SVEN 314. The H I kinematics map presents regular rotation patterns, with a constant PA at about $-90^{\circ}$. The stellar kinematics in the central few arcsec observed by Barbosa et al. (2006) with the IFU GMOS presents a kinematic PA around $130^{\circ}$. Change of orientation of the axis of rotation must then occur between the large-scale disk traced by our radio data and the innermost regions.

\section{E.11.2 NGC 5383}

NGC 5364 is a non-active spiral galaxy, hosting a large-scale stellar bar. H I moment maps are presented in Fig. D.1k, second row. The H I distribution traces very well the central regions (bulge and bar) and the outer spiral arms. Weaker $\mathrm{H}$ I intensity is observed beyond the optical image, in the continuity of the spiral arms. The H I velocity field presents a regular rotation pattern with slight wiggles in the zero-velocity line, maybe due to streaming in the bar potential. The $\mathrm{H}$ I kinematic and $\mathrm{H}$ I disk major-axes are aligned and perpendicular to the orientation of the stellar bar. Neutral hydrogen is also detected from the companion UGC 8877 located 3' south of NGC 5383. Sancisi et al. (1979) observed this galaxy with the Westerbrook array. They did not have enough sensitivity to detect the $\mathrm{H}$ I emission in the bar and in the outer regions. Apart from this, their moment maps are very similar to ours.

\section{E.12 NGC 4939/NGC5364 pair}

\section{E.12.1 NGC 4939}

This Seyfert 2 galaxy has available archive VLA data, which have not been reduced yet. 


\section{E.12.2 NGC 5364}

NGC5364 is a non-active spiral galaxy. The H I distribution traces the spiral arms of the galaxy (Fig. D.1l, second row). It is slightly asymmetric, the south side being more elongated that the north side. This elongation corresponds to the south spiral arm which is fainter and more extended than the north one. The H I kinematics is regular, with a constant PA aligned with the optical and neutral gas disk PA.

\section{E.13 NGC 5427/NGC 2967 pair}

\section{E.13.1 NGC 5427}

NGC 5427 is a SA spiral galaxy, hosting a Seyfert 2 nucleus. It is interacting with the spiral galaxy NGC 5426. This galaxy has been observed as part of the VHIKINGS project. The data reduction has not been done yet.

\section{E.13.2 NGC 2967}

NGC 2967 is an almost face-on $\left(i=24^{\circ}\right)$ SA spiral galaxy. It is a non-active galaxy, with regular H I distribution tracing the spiral arms (Fig. D. $1 \mathrm{~m}$, second row). The $\mathrm{H}$ I velocity field presents a change of orientation of the major-axis, from a PA of about $-90^{\circ}$ in the outer disk, to $\mathrm{PA} \approx-50^{\circ}$ in the inner arcmin.

\section{E.14 NGC 4941/NGC 3705 pair}

\section{E.14.1 NGC 4941}

NGC 4941 is a Seyfert 2 galaxy hosting a stellar bar. Our H I zeroth moment map (Fig. D.1n, fourth row) presents a patchy neutral gas distribution and the $\mathrm{HI}$ disk seemed to be truncated on the east side. The H I kinematics shows regular rotation patterns, aligned with the outer disk of the galaxy. The stellar kinematics in the innermost regions mapped by Barbosa et al. (2006) is in good agreement with the outer $\mathrm{H}$ I velocity fields.

\section{E.14.2 NGC 3705}

NGC 3705 is a barred spiral galaxy, classified as LINER. The galaxy disk is inclined at $68^{\circ}$. The $\mathrm{H}$ I moment maps display regular distribution and kinematics (Fig. D.1n, third row). The H I galactic disk is slightly warped at large 
scale $\left(>3^{\prime}\right)$ and a central depression is observed. H I kinematics displays a regular rotation pattern, orientated along the PA of the galaxy.

\section{E.15 NGC 4639/NGC 3162 pair}

\section{E.15.1 NGC 4639}

NGC 4639 is a barred spiral galaxy, hosting a Seyfert 1 active nucleus (Ho et al., 1997), located in the Virgo cluster. It has certainly experienced a close encounter with the companion NGC 4654 at $18^{\prime}$ south east (Vollmer, 2003).

The H I distribution of NGC 4639 (Fig. D.1o, third row) is elongated along the optical photometric PA and seems undisturbed despite the presence of NGC 4654. A central depression is observed, also noted by Cayatte et al. (1990) and Phookun \& Mundy (1995). H I emission from a small companion situated $\sim 2^{\prime}$ west of NGC 4639 is also detected. The H I kinematics is very regular with a kinematic PA aligned with the H I and optical photometric PA.

\section{E.15.2 NGC 3162}

NGC 3162 is a Sbc spiral galaxy, this is the control for NGC 4639. H I data are available in the VLA archives. We have not reduced these data yet.

\section{E.16 NGC 4258/NGC 4527 pair}

\section{E.16.1 NGC 4258 (M 106)}

NGC 4258 is a well-studied active galaxy, classified as S1.9 by Ho et al. (1997). It is highly inclined with $i=64^{\circ}$. Extensive observations at all wavelengths have been done to study this galaxy. Radio observations display spiral-arms in the central 30" (van der Kruit et al., 1972). Such features can be interpreted as the presence of a subparsec jet, close to the plane of the galaxy (Cecil et al., 2000) or to gaseous flows in a bar potential (Cox \& Downes, 1996) .

Our H I distribution map (Fig. D.1p, fourth row) shows two ring-like features, in agreement with the WSRT observations of van Albada (1980). The inner one corresponds to the optical spiral arms connected to the disk. The outer $\mathrm{H}$ I ring traces larger and fainter spiral arms, and extends beyond the optical component of the galaxy. The spiral arms observed in radio continuum in the inner regions can hardly be discerned on our emission line intensity map. Neutral gas from a companion at $\sim 10^{\prime}$ west of NGC 4258 is also detected. The $\mathrm{H}$ I velocity map displays regular rotation patterns, with a constant major-axis 
orientation, at a PA of about $-40^{\circ}$. In the central regions, some disturbance of the Hi kinematics can be observed though, where the major-axis orientation change. van Albada (1980) claimed that in these regions, H I kinematics is aligned with a bar (van Albada, 1980; Cox \& Downes, 1996).

\section{E.16.2 NGC 4527}

NGC 4527 is a Sb spiral galaxy, it is the control for NGC 4258.

NGC 4527 has been observed as part of the VHIKINGS survey, and the corresponding data have not been reduced yet.

\section{E.17 NGC 6814/NGC3963 pair}

\section{E.17.1 NGC 6814}

NGC 6814 is an almost face on spiral galaxy. It is hosting a type 1.5 active nucleus (Ho et al., 1997). Liszt \& Dickey (1995) presents VLA-D configuration H I maps of NGC 6814. They found that the neutral gas distribution present an outer ring structure, beyond the spiral arms of the galaxy, and a depression in the central regions, corresponding to the optical image. Their $\mathrm{H}$ I velocity field is regular, with a S-shape feature of the kinematic major axis, which could be evidence of the presence of outer spiral arms.

NGC 6814 has been observed as part of the VHIKINGS survey. This object has not been reduced yet.

\section{E.17.2 NGC 3963}

NGC 3963 is a non-active galaxy, hosting a large scale bar. It is dynamically associated with NGC 3958 which lies $8^{\prime}$ away to the south west. The H I distribution of NGC 3963 disk is regular, tracing the optical spiral arms (Fig. D.1q, fourth row). An extension of neutral gas towards the companion NGC 3958 is observed in the south west. Both galaxies have central H I depression. The $\mathrm{H}$ I velocity field presents complex patterns, with twists of the isovelocity contours. The global kinematic major-axes of the two galaxies NGC 3963 and 3958 are aligned. NGC 3958 displays receding velocities higher than NGC 3963. Nordgren et al. (1997) interpreted this result as NGC 6958 undergoing a direct encounter. 


\section{Bibliography}

Afanasiev, V. L., Sil'chenko, O. K., 2005, 'The Leo Triplet: Common origin or late encounter?', A\&A, 429, 825-836.

Aguerri, J. A. L., Elias-Rosa, N., Corsini, E. M., Muñoz-Tuñón, C., 2005, 'Photometric properties and origin of bulges in SB0 galaxies', A\&A, 434, 109-122.

Allard, E. L., Knapen, J. H., Peletier, R. F., Sarzi, M., 2006, 'The star formation history and evolution of the circumnuclear region of M100', MNRAS, 371, 1087-1105.

Antonucci, R., 1993, 'Unified models for active galactic nuclei and quasars', ARA\&A, 31, 473-521.

Antonucci, R. R. J., Miller, J. S., 1985, 'Spectropolarimetry and the nature of NGC 1068', ApJ, 297, 621-632.

Arribas, S., Mediavilla, E., 1994, 'Complex ionization structure and kinematics in the circumnuclear region of NGC 3227', ApJ, 437, 149-161.

Arribas, S., Mediavilla, E., Garcia-Lorenzo, B., del Burgo, C., 1997, 'Stellar and Ionized-Gas Kinematics of the Seyfert 1 Galaxy NGC 3516', ApJ, 490, 227-+.

Asif, M. W., Mundell, C. G., Pedlar, A., 2005, 'Kinematics of ionized gas in the barred Seyfert galaxy NGC 4151', MNRAS, 359, 408-416.

Asif, M. W., Mundell, C. G., Pedlar, A., Unger, S. W., Robinson, A., Vila-Vilaro, B., Lewis, J. R., 1998, 'Kinematics of the dusty circumnuclear ring in the barred Seyfert galaxy NGC 4151.', A\&A, 333, 466-470.

Athanassoula, E., 1992, 'The existence and shapes of dust lanes in galactic bars', MNRAS, 259, 345-364.

Bacon, R., Copin, Y., Monnet, G., Miller, B. W., Allington-Smith, J. R., Bureau, M., Carollo, C. M., Davies, R. L., Emsellem, E., Kuntschner, H., Peletier, R. F., Verolme, E. K., de Zeeuw, P. T., 2001, 'The SAURON project - I. The panoramic integral-field spectrograph', MNRAS, 326, 23-35.

Bahcall, J. N., Kirhakos, S., Schneider, D. P., 1995, 'PKS 2349-014: A Luminous Quasar with Thin Wisps, a Large Off-Center Nebulosity, and a Close Companion Galaxy', ApJ, 447, L1+. 
Barberà, C., Athanassoula, E., García-Gómez, C., 2004, ‘Deprojecting spiral galaxies using Fourier analysis. Application to the Frei sample', A\&A, 415, 849-861.

Barbosa, F. K. B., Storchi-Bergmann, T., Cid Fernandes, R., Winge, C., Schmitt, H., 2006, 'Gemini/GMOS Integral Field Unit stellar kinematics of the nuclear region of six nearby active galaxies', MNRAS, 371, 170-184.

Barnes, J. E., Hernquist, L. E., 1991, 'Fueling starburst galaxies with gas-rich mergers', ApJ, 370, L65-L68.

Battaglia, G., Fraternali, F., Oosterloo, T., Sancisi, R., 2006, 'ion $\{\mathrm{H}\}\{\mathrm{i}\}$ study of the warped spiral galaxy NGC 5055: a disk/dark matter halo offset?', A\&A, $447,49-62$.

Begeman, K. G., 1987. Ph.D. thesis, , Kapteyn Institute, (1987).

Bender, R., 1990, 'Unraveling the kinematics of early-type galaxies - Presentation of a new method and its application to NGC4621', A\&A, 229, 441-451.

Bertola, F., Buson, L. M., Zeilinger, W. W., 1992, 'The external origin of the gas in S0 galaxies', ApJ, 401, L79-L81.

Binney, J., Tremaine, S., 1987, 'Galactic dynamics', Princeton, NJ, Princeton University Press, 1987, $747 \mathrm{p}$.

Blais-Ouellette, S., Amram, P., Carignan, C., Swaters, R., 2004, 'Accurate determination of the mass distribution in spiral galaxies. III. Fabry-Perot imaging spectroscopy of 6 spiral galaxies', A\&A, 420, 147-161.

Bland-Hawthorn, J., Gallimore, J. F., Tacconi, L. J., Brinks, E., Baum, S. A., Antonucci, R. R. J., Cecil, G. N., 1997, 'The Ringberg Standards for NGC 1068', Ap\&SS, 248, 9-19.

Blandford, R. D., McKee, C. F., 1982, 'Reverberation mapping of the emission line regions of Seyfert galaxies and quasars', ApJ, 255, 419-439.

Blandford, R. D., Netzer, H., Woltjer, L., Courvoisier, T. J.-L., Mayor, M., 1990, 'Active Galactic Nuclei', Saas-Fee Advanced Course 20. Lecture Notes 1990. Swiss Society for Astrophysics and Astronomy, XII, 280 pp. 97 figs.. Springer-Verlag Berlin Heidelberg New York.

Boyle, B. J., Shanks, T., Croom, S. M., Smith, R. J., Miller, L., Loaring, N., Heymans, C., 2000, 'The 2dF QSO Redshift Survey - I. The optical luminosity function of quasi-stellar objects', MNRAS, 317, 1014-1022.

Cappellari, M., Bacon, R., Bureau, M., Damen, M. C., Davies, R. L., de Zeeuw, P. T., Emsellem, E., Falcón-Barroso, J., Krajnović, D., Kuntschner, H., McDermid, R. M., Peletier, R. F., Sarzi, M., van den Bosch, R. C. E., van de Ven, G., 2006, 'The SAURON project - IV. The mass-to-light ratio, the virial mass estimator and the Fundamental Plane of elliptical and lenticular galaxies', MNRAS, 366, 1126-1150. 
Cappellari, M., Copin, Y., 2003, 'Adaptive spatial binning of integral-field spectroscopic data using Voronoi tessellations', MNRAS, 342, 345-354.

Cappellari, M., Emsellem, E., 2004, ‘Parametric Recovery of Line-of-Sight Velocity Distributions from Absorption-Line Spectra of Galaxies via Penalized Likelihood', PASP, 116, 138-147.

Cappellari, M., Emsellem, E., Bacon, R., Bureau, M., Davies, R. L., de Zeeuw, P. T., Falcón-Barroso, J., Krajnović, D., Kuntschner, H., McDermid, R. M., Peletier, R. F., Sarzi, M., van den Bosch, R. C. E., van de Ven, G., 2007, 'The SAURON project - $X$. The orbital anisotropy of elliptical and lenticular galaxies: revisiting the $(\mathrm{V} / \sigma, \epsilon)$ diagram with integral-field stellar kinematics', MNRAS, 379, 418-444.

Carollo, C. M., Stiavelli, M., Seigar, M., de Zeeuw, P. T., Dejonghe, H., 2002, 'Spiral Galaxies with HST/NICMOS. I. Nuclear Morphologies, Color Maps, and Distinct Nuclei', AJ, 123, 159-183.

Cayatte, V., van Gorkom, J. H., Balkowski, C., Kotanyi, C., 1990, 'VLA observations of neutral hydrogen in Virgo Cluster galaxies. I - The Atlas', AJ, 100, 604-634.

Cecil, G., Greenhill, L. J., DePree, C. G., Nagar, N., Wilson, A. S., Dopita, M. A., Pérez-Fournon, I., Argon, A. L., Moran, J. M., 2000, 'The Active Jet in NGC 4258 and Its Associated Shocks', ApJ, 536, 675-696.

Chemin, L., Cayatte, V., Balkowski, C., Marcelin, M., Amram, P., van Driel, W., Flores, H., 2003, 'An H $\alpha$ study of the kinematics of NGC 3627', A\&A, 405, 89-97.

Chiaberge, M., Capetti, A., Macchetto, F. D., 2005, 'The Hubble Space Telescope View of LINER Nuclei: Evidence for a Dual Population?', ApJ, 625, 716-726.

Christopoulou, P. E., Holloway, A. J., Steffen, W., Mundell, C. G., Thean, A. H. C., Goudis, C. D., Meaburn, J., Pedlar, A., 1997, 'Evidence for an outflow from the Seyfert galaxy NGC 4051', MNRAS, 284, 385-394.

Collin-Souffrin, S., Czerny, B., Dumont, A.-M., Zycki, P. T., 1996, 'Quasispherical accretion of optically thin clouds as a model for the opti$\mathrm{cal} / \mathrm{UV} /$ soft X-ray emission of AGN.', A\&A, 314, 393-413.

Combes, F., 2001, 'Fueling the AGN', In: Aretxaga, I., Kunth, D., Mújica, R. (Eds.), Advanced Lectures on the Starburst-AGN, pp. 223-+.

Combes, F., 2006. Formation and Evolution of Supermassive Black Holes. Astrophysics Update 2, pp. 159-+.

Contopoulos, G., Papayannopoulos, T., 1980, 'Orbits in weak and strong bars', A\&A, 92, 33-46. 
Corsini, E. M., Debattista, V. P., Aguerri, J. A. L., 2003, ‘Direct Confirmation of Two Pattern Speeds in the Double-barred Galaxy NGC 2950', ApJ, 599, L29-L32.

Cox, P., Downes, D., 1996, 'CO Observations and a New Interpretation of the Anomalous Arms of NGC 4258', ApJ, 473, 219-+.

Crane, P. C., van der Hulst, J. M., 1992, 'The radio jet in M51', AJ, 103, 11461150.

Croom, S. M., Smith, R. J., Boyle, B. J., Shanks, T., Miller, L., Outram, P. J., Loaring, N. S., 2004, 'The 2dF QSO Redshift Survey - XII. The spectroscopic catalogue and luminosity function', MNRAS, 349, 1397-1418.

Davies, R. I., Thomas, J., Genzel, R., Mueller Sánchez, F., Tacconi, L. J., Sternberg, A., Eisenhauer, F., Abuter, R., Saglia, R., Bender, R., 2006, 'The Starforming Torus and Stellar Dynamical Black Hole Mass in the Seyfert $1 \mathrm{Nu}$ cleus of NGC 3227', ApJ, 646, 754-773.

de Blok, W. J. G., Walter, F., Brinks, E., Thornley, M. D., Kennicutt, Jr., R. C., 2005, 'First Results from THINGS: The HI Nearby Galaxy Survey', In: Fairall, A. P., Woudt, P. A. (Eds.), Nearby Large-Scale Structures and the Zone of Avoidance, Vol. 329 of Astronomical Society of the Pacific Conference Series, pp. 265-+.

De Robertis, M. M., Yee, H. K. C., Hayhoe, K., 1998, 'A CCD Study of the Environment of Seyfert Galaxies. II. Testing the Interaction Hypothesis', ApJ, 496, 93-+.

de Zeeuw, P. T., Bureau, M., Emsellem, E., Bacon, R., Carollo, C. M., Copin, Y., Davies, R. L., Kuntschner, H., Miller, B. W., Monnet, G., Peletier, R. F., Verolme, E. K., 2002, 'The SAURON project - II. Sample and early results', MNRAS, 329, 513-530.

Devereux, N. A., Kenney, J. D., Young, J. S., 1992, 'Nuclear molecular gas bar perpendicular to the stellar bar in NGC 3351', AJ, 103, 784-792.

Elmegreen, D. M., Chromey, F. R., Bissell, B. A., Corrado, K., 1999, 'K'-Band Observations of Underlying Symmetric Structure in Flocculent Galaxies', AJ, $118,2618-2624$.

Emsellem, E., 2006, 'The origin of sigma-drops: mapping stellar kinematics and populations in spirals', ArXiv Astrophysics e-prints.

Emsellem, E., Cappellari, M., Krajnović, D., van de Ven, G., Bacon, R., Bureau, M., Davies, R. L., de Zeeuw, P. T., Falcón-Barroso, J., Kuntschner, H., McDermid, R., Peletier, R. F., Sarzi, M., 2007, 'The SAURON project - IX. A kinematic classification for early-type galaxies', MNRAS, 379, 401-417.

Emsellem, E., Cappellari, M., Peletier, R. F., McDermid, R. M., Bacon, R., Bureau, M., Copin, Y., Davies, R. L., Krajnović, D., Kuntschner, H., Miller, B. W., 
de Zeeuw, P. T., 2004, 'The SAURON project - III. Integral-field absorptionline kinematics of 48 elliptical and lenticular galaxies', MNRAS, 352, 721743.

Emsellem, E., Fathi, K., Wozniak, H., Ferruit, P., Mundell, C. G., Schinnerer, E., 2006, 'Gas and stellar dynamics in NGC 1068: probing the galactic gravitational potential', MNRAS, 365, 367-384.

Emsellem, E., Greusard, D., Combes, F., Friedli, D., Leon, S., Pécontal, E., Wozniak, H., 2001, 'Dynamics of embedded bars and the connection with AGN. I. ISAAC/VLT stellar kinematics', A\&A, 368, 52-63.

Englmaier, P., Shlosman, I., 2001, ‘Driving a Gaseous Nuclear Spiral Inside the Inner LindBlad Resonance', Ap\&SS, 276, 707-714.

Erwin, P., 2005, 'How large are the bars in barred galaxies?', MNRAS, 364, 283-302.

Erwin, P., Sparke, L. S., 2002, ‘Double Bars, Inner Disks, and Nuclear Rings in Early-Type Disk Galaxies', AJ, 124, 65-77.

Erwin, P., Sparke, L. S., 2003, 'An Imaging Survey of Early-Type Barred Galaxies', ApJS, 146, 299-352.

Falcón-Barroso, J., Bacon, R., Bureau, M., Cappellari, M., Davies, R. L., de Zeeuw, P. T., Emsellem, E., Fathi, K., Krajnović, D., Kuntschner, H., McDermid, R. M., Peletier, R. F., Sarzi, M., 2006, 'The SAURON project - VII. Integral-field absorption and emission-line kinematics of 24 spiral galaxy bulges', MNRAS, 369, 529-566.

Fan, X., Strauss, M. A., Schneider, D. P., Becker, R. H., White, R. L., Haiman, Z., Gregg, M., Pentericci, L., Grebel, E. K., Narayanan, V. K., Loh, Y.-S., Richards, G. T., Gunn, J. E., Lupton, R. H., Knapp, G. R., Ivezić, Ž., Brandt, W. N., Collinge, M., Hao, L., Harbeck, D., Prada, F., Schaye, J., Strateva, I., Zakamska, N., Anderson, S., Brinkmann, J., Bahcall, N. A., Lamb, D. Q., Okamura, S., Szalay, A., York, D. G., 2003, 'A Survey of z>5.7 Quasars in the Sloan Digital Sky Survey. II. Discovery of Three Additional Quasars at z>6', AJ, 125, 1649-1659.

Fanaroff, B. L., Riley, J. M., 1974, 'The morphology of extragalactic radio sources of high and low luminosity', MNRAS, 167, 31P-36P.

Fathi, K., Peletier, R. F., 2003, 'Do bulges of early- and late-type spirals have different morphology?', A\&A, 407, 61-74.

Fathi, K., van de Ven, G., Peletier, R. F., Emsellem, E., Falcón-Barroso, J., Cappellari, M., de Zeeuw, T., 2005, 'A bar signature and central disc in the gaseous and stellar velocity fields of NGC 5448', MNRAS, 364, 773-782. 
Ferrarese, L., Côté, P., Dalla Bontà, E., Peng, E. W., Merritt, D., Jordán, A., Blakeslee, J. P., Haşegan, M., Mei, S., Piatek, S., Tonry, J. L., West, M. J., 2006, 'A Fundamental Relation between Compact Stellar Nuclei, Supermassive Black Holes, and Their Host Galaxies', ApJ, 644, L21-L24.

Ferrarese, L., Merritt, D., 2000, 'A Fundamental Relation between Supermassive Black Holes and Their Host Galaxies', ApJ, 539, L9-L12.

Ferruit, P., Mundell, C. G., Nagar, N. M., Emsellem, E., Pécontal, E., Wilson, A. S., Schinnerer, E., 2004, 'Ionized gas and stars in the central kiloparsec of the type 2 Seyfert galaxy NGC 2110 - I. The data', MNRAS, 352, 1180-1198.

Ford, H. C., Crane, P. C., Jacoby, G. H., Lawrie, D. G., van der Hulst, J. M., 1985, 'Bubbles and jets in the center of M51', ApJ, 293, 132-147.

Friedli, D., Benz, W., 1993, 'Secular evolution of isolated barred galaxies. I Gravitational coupling between stellar bars and interstellar medium', A\&A, $268,65-85$.

Gallimore, J. F., Axon, D. J., O'Dea, C. P., Baum, S. A., Pedlar, A., 2006, 'A Survey of Kiloparsec-Scale Radio Outflows in Radio-Quiet Active Galactic Nuclei', AJ, 132, 546-569.

Gallimore, J. F., Baum, S. A., O’Dea, C. P., Brinks, E., Pedlar, A., 1996, 'H 2 O and $\mathrm{OH}$ Masers as Probes of the Obscuring Torus in NGC 1068', ApJ, 462, 740-+.

Ganda, K., Falcón-Barroso, J., Peletier, R. F., Cappellari, M., Emsellem, E., McDermid, R. M., de Zeeuw, P. T., Carollo, C. M., 2006, 'Late-type galaxies observed with SAURON: two-dimensional stellar and emission-line kinematics of 18 spirals', MNRAS, 367, 46-78.

García-Burillo, S., Combes, F., Hunt, L. K., Boone, F., Baker, A. J., Tacconi, L. J., Eckart, A., Neri, R., Leon, S., Schinnerer, E., Englmaier, P., 2003, 'Molecular Gas in NUclei of GAlaxies (NUGA). I. The counter-rotating LINER NGC 4826', A\&A, 407, 485-502.

García-Burillo, S., Combes, F., Schinnerer, E., Boone, F., Hunt, L. K., 2005, 'Molecular gas in NUclei of GAlaxies (NUGA). IV. Gravitational torques and AGN feeding', A\&A, 441, 1011-1030.

García-Gómez, C., Athanassoula, E., Barberà, C., 2002, 'Analysis of the distribution of HII regions in external galaxies. IV. The new galaxy sample. Position and inclination angles', A\&A, 389, 68-83.

Garcia-Lorenzo, B., Mediavilla, E., Arribas, S., del Burgo, C., 1997, 'Evidence of Two Kinematically Different Stellar Systems in NGC 1068’, ApJ, 483, L99+. 
Gebhardt, K., Bender, R., Bower, G., Dressler, A., Faber, S. M., Filippenko, A. V., Green, R., Grillmair, C., Ho, L. C., Kormendy, J., Lauer, T. R., Magorrian, J., Pinkney, J., Richstone, D., Tremaine, S., 2000, 'A Relationship between Nuclear Black Hole Mass and Galaxy Velocity Dispersion', ApJ, 539, L13-L16.

Genzel, R., Pichon, C., Eckart, A., Gerhard, O. E., Ott, T., 2000, 'Stellar dynamics in the Galactic Centre: proper motions and anisotropy', MNRAS, 317, 348-374.

Gerhard, O. E., 1993, 'Line-of-sight velocity profiles in spherical galaxies: breaking the degeneracy between anisotropy and mass.', MNRAS, 265, 213+ .

Gerssen, J., Allington-Smith, J., Miller, B. W., Turner, J. E. H., Walker, A., 2006, 'GMOS IFU observations of the stellar and gaseous kinematics in the centre of NGC 1068', MNRAS, 365, 29-36.

González-Martín, O., Masegosa, J., Márquez, I., Guerrero, M. A., DultzinHacyan, D., 2006, 'X-ray nature of the LINER nuclear sources', A\&A, 460, $45-57$.

Groves, B. A., Dopita, M. A., Sutherland, R. S., 2004, 'Dusty, Radiation Pressure-Dominated Photoionization. I. Model Description, Structure, and Grids', ApJS, 153, 9-73.

Guhathakurta, P., van Gorkom, J. H., Kotanyi, C. G., Balkowski, C., 1988, 'A VLA H I survey of the Virgo cluster spirals. II - Rotation curves', AJ, 96, 851-866.

Haan, S., Schinnerer, E., Mundell, C. G., Garcia-Burillo, S., Combes, F., 2007, 'Atomic Hydrogen Properties of AGN Host Galaxies: HI in 16 NUclei of GAlaxies (NUGA) Sources', ArXiv e-prints, 710.

Haas, M., Klaas, U., Müller, S. A. H., Bertoldi, F., Camenzind, M., Chini, R., Krause, O., Lemke, D., Meisenheimer, K., Richards, P. J., Wilkes, B. J., 2003, 'The ISO view of Palomar-Green quasars', A\&A, 402, 87-111.

Hasinger, G., 2005, 'When Supermassive Black Holes Were Growing: Clues from Deep X-ray Surveys', In: Merloni, A., Nayakshin, S., Sunyaev, R. A. (Eds.), Growing Black Holes: Accretion in a Cosmological Context, pp. 418-431.

Haynes, M. P., 1981, ‘Neutral hydrogen streams in groups of galaxies. I - Observations', AJ, 86, 1126-1154.

Heckman, T. M., 1980, 'An optical and radio survey of the nuclei of bright galaxies - Activity in normal galactic nuclei', A\&A, 87, 152-164.

Heckman, T. M., 1990, 'Galaxy interactions and the stimulation of nuclear activity', In: Sulentic, J. W., Keel, W. C., Telesco, C. M. (Eds.), IAU Colloq. 124: Paired and Interacting Galaxies, pp. 359-382. 
Heckman, T. M., Kauffmann, G., Brinchmann, J., Charlot, S., Tremonti, C., White, S. D. M., 2004, 'Present-Day Growth of Black Holes and Bulges: The Sloan Digital Sky Survey Perspective', ApJ, 613, 109-118.

Hernandez, O., Carignan, C., Amram, P., Chemin, L., Daigle, O., 2005, 'BH $\alpha$ BAR: big $\mathrm{H} \alpha$ kinematical sample of barred spiral galaxies - I. FabryPerot observations of 21 galaxies', MNRAS, 360, 1201-1230.

Hernquist, L., Mihos, J. C., 1995, 'Excitation of Activity in Galaxies by Minor Mergers', ApJ, 448, 41-+.

Ho, L. C., Filippenko, A. V., Sargent, W. L. W., 1997, 'A Search for "Dwarf" Seyfert Nuclei. III. Spectroscopic Parameters and Properties of the Host Galaxies', ApJS, 112, 315-+.

Ho, L. C., Filippenko, A. V., Sargent, W. L. W., 2003, 'A Search for "Dwarf" Seyfert Nuclei. VI. Properties of Emission-Line Nuclei in Nearby Galaxies', ApJ, 583, 159-177.

Hopkins, P. F., Hernquist, L., 2006, 'Fueling Low-Level AGN Activity through Stochastic Accretion of Cold Gas', ApJS, 166, 1-36.

Huchtmeier, W. K., Richter, O.-G., 1982, 'Extended H I-envelopes around spiral galaxies - NGC 2655 and NGC 2715', A\&A, 109, 331-335.

Hunt, L. K., Malkan, M. A., 2004, 'Circumnuclear Structure and Black Hole Fueling: Hubble Space Telescope NICMOS Imaging of 250 Active and Normal Galaxies', ApJ, 616, 707-729.

Hutchings, J. B., Neff, S. G., 1988, 'Tidal interactions and infrared-bright QSOs', AJ, 96, 1575-1580.

Jaffe, W., Ford, H., Ferrarese, L., van den Bosch, F., O'Connell, R. W., 1996, 'The Nuclear Disk of NGC 4261: Hubble Space Telescope Images and Groundbased Spectra', ApJ, 460, 214-+.

Jaffe, W., Meisenheimer, K., Röttgering, H. J. A., Leinert, C., Richichi, A., Chesneau, O., Fraix-Burnet, D., Glazenborg-Kluttig, A., Granato, G.-L., Graser, U., Heijligers, B., Köhler, R., Malbet, F., Miley, G. K., Paresce, F., Pel, J.-W., Perrin, G., Przygodda, F., Schoeller, M., Sol, H., Waters, L. B. F. M., Weigelt, G., Woillez, J., de Zeeuw, P. T., 2004, 'The central dusty torus in the active nucleus of NGC 1068', Nature, 429, 47-49.

Jarrett, T. H., Chester, T., Cutri, R., Schneider, S. E., Huchra, J. P., 2003, 'The 2MASS Large Galaxy Atlas', AJ, 125, 525-554.

Jogee, S., Knapen, J. H., Laine, S., Shlosman, I., Scoville, N. Z., Englmaier, P., 2002a, 'Discovery and Implications of a New Large-Scale Stellar Bar in NGC 5248’, ApJ, 570, L55-L59. 
Jogee, S., Scoville, N., Kenney, J. D. P., 2005, 'The Central Region of Barred Galaxies: Molecular Environment, Starbursts, and Secular Evolution', ApJ, 630, 837-863.

Jogee, S., Shlosman, I., Laine, S., Englmaier, P., Knapen, J. H., Scoville, N., Wilson, C. D., 2002b, 'Gasdynamics in NGC 5248: Fueling a Circumnuclear Starburst Ring of Super-Star Clusters', ApJ, 575, 156-177.

Jore, K. P., Broeils, A. H., Haynes, M. P., 1996, 'A Counter-Rotating Disk in the Normal SA Galaxy NGC 4138', AJ, 112, 438-+.

Jourdain, E., Collin, S., Dennefeld, M., Ballet, J., Roques, J. P., Lebrun, F., Mandrou, P., Cordier, B., Finoguenov, A., Churazof, E., Gilfanov, M., Sunyaev, R., Kuleshova, N., Sheikhet, A., Sukhanov, K., Tserenin, I., 1994, 'NGC 1068: A genuine hidden Seyfert 1 galaxy?', A\&A, 281, L57-L60.

Kaspi, S., Maoz, D., Netzer, H., Peterson, B. M., Vestergaard, M., Jannuzi, B. T., 2005, 'The Relationship between Luminosity and Broad-Line Region Size in Active Galactic Nuclei', ApJ, 629, 61-71.

Kaspi, S., Smith, P. S., Netzer, H., Maoz, D., Jannuzi, B. T., Giveon, U., 2000, 'Reverberation Measurements for 17 Quasars and the Size-Mass-Luminosity Relations in Active Galactic Nuclei', ApJ, 533, 631-649.

Kassin, S. A., de Jong, R. S., Pogge, R. W., 2006, 'Dark and Baryonic Matter in Bright Spiral Galaxies. I. Near-Infrared and Optical Broadband Surface Photometry of 30 Galaxies', ApJS, 162, 80-96.

Kauffmann, G., Heckman, T. M., Tremonti, C., Brinchmann, J., Charlot, S., White, S. D. M., Ridgway, S. E., Brinkmann, J., Fukugita, M., Hall, P. B., Ivezić, Ž., Richards, G. T., Schneider, D. P., 2003, ‘The host galaxies of active galactic nuclei', MNRAS, 346, 1055-1077.

Keel, W. C., Hummel, E., 1988, 'Jets and loops from the nucleus of NGC 2655', A\&A, 194, 90-98.

Kellermann, K. I., Sramek, R., Schmidt, M., Shaffer, D. B., Green, R., 1989, 'VLA observations of objects in the Palomar Bright Quasar Survey', AJ, 98, 11951207.

Kellermann, K. I., Vermeulen, R. C., Zensus, J. A., Cohen, M. H., 1998, 'SubMilliarcsecond Imaging of Quasars and Active Galactic Nuclei', AJ, 115, 1295-1318.

Kewley, L. J., Groves, B., Kauffmann, G., Heckman, T., 2006, 'The host galaxies and classification of active galactic nuclei', MNRAS, 372, 961-976.

Kewley, L. J., Heisler, C. A., Dopita, M. A., Lumsden, S., 2001, 'Optical Classification of Southern Warm Infrared Galaxies', ApJS, 132, 37-71.

Kewley, L. J., Jansen, R. A., Geller, M. J., 2005, 'A perture Effects on Star Formation Rate, Metallicity, and Reddening', PASP, 117, 227-244. 
Kim, D.-W., Fabbiano, G., Trinchieri, G., 1992, 'The X-ray spectra of galaxies. I - Spectral FITS of individual galaxies and X-ray colors', ApJS, 80, 645-681.

King, A. R., Pringle, J. E., 2006, ‘Growing supermassive black holes by chaotic accretion', MNRAS, 373, L90-L92.

King, A. R., Pringle, J. E., 2007, 'Fuelling active galactic nuclei', MNRAS, 377, L25-L28.

Kinney, A. L., Schmitt, H. R., Clarke, C. J., Pringle, J. E., Ulvestad, J. S., Antonucci, R. R. J., 2000, 'Jet Directions in Seyfert Galaxies', ApJ, 537, 152-177.

Kirhakos, S., Bahcall, J. N., Schneider, D. P., Kristian, J., 1999, 'The Host Galaxies of Three Radio-loud Quasars: 3C 48, 3C 345, and B2 1425+267', ApJ, 520, $67-77$.

Knapen, J. H., Mazzuca, L. M., Böker, T., Shlosman, I., Colina, L., Combes, F., Axon, D. J., 2006, 'Massive star formation in the central regions of spiral galaxies', A\&A, 448, 489-498.

Knapen, J. H., Shlosman, I., Peletier, R. F., 2000, 'A Subarcsecond Resolution Near-Infrared Study of Seyfert and "Normal" Galaxies. II. Morphology', ApJ, 529, 93-100.

Koratkar, A., Blaes, O., 1999, 'The Ultraviolet and Optical Continuum Emission in Active Galactic Nuclei: The Status of Accretion Disks', PASP, 111, 1-30.

Koribalski, B., Dettmar, R.-J., Mebold, U., Wielebinski, R., 1996, 'Gas streaming along the bar in NGC 1808. A combination of high-resolution $\mathrm{H} \alpha$ and $\mathrm{HI}$ data.', A\&A, 315, 71-76.

Krajnović, D., Cappellari, M., de Zeeuw, P. T., Copin, Y., 2006, 'Kinemetry: a generalization of photometry to the higher moments of the line-of-sight velocity distribution', MNRAS, 366, 787-802.

Krolik, J. H., 1999, 'Active galactic nuclei : from the central black hole to the galactic environment', Active galactic nuclei: from the central black hole to the galactic environment/Julian H. Krolik. Princeton, N. J. : Princeton University Press, c1999.

Kuntschner, H., Emsellem, E., Bacon, R., Bureau, M., Cappellari, M., Davies, R. L., de Zeeuw, P. T., Falcón-Barroso, J., Krajnović, D., McDermid, R. M., Peletier, R. F., Sarzi, M., 2006, 'The SAURON project - VI. Line strength maps of 48 elliptical and lenticular galaxies', MNRAS, 369, 497-528.

Laine, S., Knapen, J. H., Pérez-Ramírez, D., Englmaier, P., Matthias, M., 2001, 'Circumnuclear kinematics in NGC 5248: the origin of nuclear spiral arms', MNRAS, 324, 891-898.

Laine, S., Shlosman, I., Knapen, J. H., Peletier, R. F., 2002, ‘Nested and Single Bars in Seyfert and Non-Seyfert Galaxies', ApJ, 567, 97-117. 
Laor, A., Netzer, H., 1989, 'Massive thin accretion discs. I - Calculated spectra', MNRAS, 238, 897-916.

Laurikainen, E., Salo, H., 1995, 'Environments of Seyfert galaxies. II. Statistical analyses.', A\&A, 293, 683-702.

Laurikainen, E., Salo, H., Buta, R., 2004a, 'Comparison of Bar Strengths and Fractions of Bars in Active and Nonactive Galaxies', ApJ, 607, 103-124.

Laurikainen, E., Salo, H., Buta, R., Vasylyev, S., 2004b, 'Bar-induced perturbation strengths of the galaxies in the Ohio State University Bright Galaxy Survey - I', MNRAS, 355, 1251-1271.

Liszt, H. S., Dickey, J. M., 1995, ‘HI Emission From the Seyfert Galaxies NGC 4051 and NGC 6814', AJ, 110, 998-+.

Lynden-Bell, D., 1969, 'Galactic Nuclei as Collapsed Old Quasars', Nature, 223, 690-+.

Maciejewski, W., 2004a, 'Nuclear spirals in galaxies: gas response to an asymmetric potential - I. Linear theory', MNRAS, 354, 883-891.

Maciejewski, W., 2004b, 'Nuclear spirals in galaxies: gas response to an asymmetric potential - II. Hydrodynamical models', MNRAS, 354, 892-904.

Maciejewski, W., Sparke, L. S., 1997, 'Regular Orbits and Periodic Loops in Multiply-Barred Galactic Potentials', ApJ, 484, L117+.

Maciejewski, W., Teuben, P. J., Sparke, L. S., Stone, J. M., 2002, 'Gas inflow in barred galaxies - effects of secondary bars', MNRAS, 329, 502-512.

Malkan, M. A., Sargent, W. L. W., 1982, 'The ultraviolet excess of Seyfert 1 galaxies and quasars', ApJ, 254, 22-37.

Márquez, I., Masegosa, J., Durret, F., González Delgado, R. M., Moles, M., Maza, J., Pérez, E., Roth, M., 2003, 'The detection of stellar velocity dispersion drops in the central regions of five isolated Seyfert spirals', A\&A, 409, 459-467.

Marquez, I., Moles, M., 1993, 'The stellar content, star formation, and mass of the active galaxy NGC 6951', AJ, 105, 2090-2097.

Martini, P., Pogge, R. W., 1999, 'Hubble Space Telescope Observations of the CFA Seyfert 2 Galaxies: The Fueling of Active Galactic Nuclei', AJ, 118, 26462657.

Martini, P., Regan, M. W., Mulchaey, J. S., Pogge, R. W., 2003, 'Circumnuclear Dust in Nearby Active and Inactive Galaxies. II. Bars, Nuclear Spirals, and the Fueling of Active Galactic Nuclei', ApJ, 589, 774-782.

Mediavilla, E., Arribas, S., 1993, 'Evidence for an offset active nucleus in the Seyfert galaxy NGC 3227.', Nature, 365, 420-422. 
Mediavilla, E., Arribas, S., 1995, 'Two-dimensional spectroscopy of the Seyfert galaxies NGC 5728 and 4151 in the [OIII] lambdalambda4959, 5007 and $\mathrm{H} \alpha$ spectral ranges', MNRAS, 276, 579-591.

Mihos, J. C., Hernquist, L., 1996, 'Gasdynamics and Starbursts in Major Mergers', ApJ, 464, 641-+.

Miyoshi, M., Moran, J., Herrnstein, J., Greenhill, L., Nakai, N., Diamond, P., Inoue, M., 1995, 'Evidence for a Black-Hole from High Rotation Velocities in a Sub-Parsec Region of NGC4258', Nature, 373, 127-+.

Mulchaey, J. S., Regan, M. W., 1997, 'The Fueling of Nuclear Activity: The Bar Properties of Seyfert and Normal Galaxies', ApJ, 482, L135+.

Mundell, C. G., Dumas, G., Schinnerer, E., Nagar, N., Haan, S., Wilcots, E., Wilson, A. S., Emsellem, E., Ferruit, P., Peletier, R. F., de Zeeuw, P. T., 2007, '3D studies of neutral and ionised gas and stars in seyfert and inactive galaxies', New AR, 51, 34-37.

Mundell, C. G., Holloway, A. J., Pedlar, A., Meaburn, J., Kukula, M. J., Axon, D. J., 1995a, 'Anisotropic radio and optical emission in the Seyfert nucleus of NGC 3227', MNRAS, 275, 67-75.

Mundell, C. G., James, P. A., Loiseau, N., Schinnerer, E., Forbes, D. A., 2004, 'The Unusual Tidal Dwarf Candidate in the Merger System NGC 3227/3226: Star Formation in a Tidal Shock?', ApJ, 614, 648-657.

Mundell, C. G., Pedlar, A., Axon, D. J., Meaburn, J., Unger, S. W., 1995b, ‘Neutral hydrogen studies of the Seyfert galaxy NGC 3227', MNRAS, 277, 641654.

Mundell, C. G., Pedlar, A., Shone, D. L., Robinson, A., 1999, 'Gas dynamics in the barred Seyfert galaxy NGC 4151 - II. High-resolution HI study', MNRAS, 304, 481-494.

Mundell, C. G., Shone, D. L., 1999, 'Gas dynamics in the barred Seyfert galaxy NGC 4151 - I. HI streaming shocks and inflow along the bar', MNRAS, 304, 475-480.

Mundell, C. G., Wrobel, J. M., Pedlar, A., Gallimore, J. F., 2003, 'The Nuclear Regions of the Seyfert Galaxy NGC 4151: Parsec-Scale H I Absorption and a Remarkable Radio Jet', ApJ, 583, 192-204.

Muxlow, T. W. B., Pedlar, A., Holloway, A. J., Gallimore, J. F., Antonucci, R. R. J., 1996, 'The compact radio nucleus of the Seyfert galaxy NGC 1068', MNRAS, 278, 854-860.

Nagar, N. M., Falcke, H., Wilson, A. S., 2005, 'Radio sources in low-luminosity active galactic nuclei. IV. Radio luminosity function, importance of jet power, and radio properties of the complete Palomar sample', A\&A, 435, 521-543. 
Nagar, N. M., Wilson, A. S., 1999, 'The Relative Orientation of Nuclear Accretion and Galaxy Stellar Disks in Seyfert Galaxies', ApJ, 516, 97-113.

Nayakshin, S., Melia, F., 1997, 'Big Blue Bump and Transient Active Regions in Seyfert Galaxies', ApJ, 484, L103+.

Neugebauer, G., Miley, G. K., Soifer, B. T., Clegg, P. E., 1986, 'Quasars measured by the Infrared Astronomical Satellite', ApJ, 308, 815-828.

Noordermeer, E., van der Hulst, J. M., Sancisi, R., Swaters, R. A., van Albada, T. S., 2005, 'The Westerbork HI survey of spiral and irregular galaxies. III. HI observations of early-type disk galaxies', A\&A, 442, 137-157.

Nordgren, T. E., Chengalur, J. N., Salpeter, E. E., Terzian, Y., 1997, ‘Close Galaxy pairs in Medium Density Regions: The Northern Sky', AJ, 114, 77-93.

Oliva, E., Marconi, A., Moorwood, A. F. M., 1999, 'Metal abundances and excitation of extranuclear clouds in the Circinus galaxy. A new method for deriving abundances of AGN narrow line clouds', A\&A, 342, 87-100.

Osterbrock, D. E., 1981, 'Seyfert galaxies with weak broad H alpha emission lines', ApJ, 249, 462-470.

Osterbrock, D. E., 1993, 'The nature and structure of active galactic nuclei', ApJ, 404, 551-562.

Panessa, F., Bassani, L., Cappi, M., Dadina, M., Barcons, X., Carrera, F. J., Ho, L. C., Iwasawa, K., 2006, 'On the X-ray, optical emission line and black hole mass properties of local Seyfert galaxies', A\&A, 455, 173-185.

Pedlar, A., Howley, P., Axon, D. J., Unger, S. W., 1992, 'A neutral hydrogen study of NGC 4151', MNRAS, 259, 369-380.

Peeples, M. S., Martini, P., 2006, 'The Connection between Bar Strength and Circumnuclear Dust Structure', ApJ, 652, 1097-1111.

Perez, E., Gonzalez-Delgado, R., Tadhunter, C., Tsvetanov, Z., 1989, 'The complex narrow-line region in NGC 4151', MNRAS, 241, 31P-36P.

Pérez, E., Márquez, I., Marrero, I., Durret, F., González Delgado, R. M., Masegosa, J., Maza, J., Moles, M., 2000, 'Circumnuclear structure and kinematics in the active galaxy NGC 6951', A\&A, 353, 893-909.

Pérez-Ramírez, D., Knapen, J. H., Peletier, R. F., Laine, S., Doyon, R., Nadeau, D., 2000, 'Circumnuclear regions in barred spiral galaxies - I. Near-infrared imaging', MNRAS, 317, 234-248.

Perley, R. A., Dreher, J. W., Cowan, J. J., 1984, 'The jet and filaments in Cygnus A', ApJ, 285, L35-L38. 
Peterson, B. M., Ferrarese, L., Gilbert, K. M., Kaspi, S., Malkan, M. A., Maoz, D., Merritt, D., Netzer, H., Onken, C. A., Pogge, R. W., Vestergaard, M., Wandel, A., 2004, 'Central Masses and Broad-Line Region Sizes of Active Galactic Nuclei. II. A Homogeneous Analysis of a Large Reverberation-Mapping Database', ApJ, 613, 682-699.

Phookun, B., Mundy, L. G., 1995, 'NGC 4654: A Virgo Cluster Spiral Interacting with the Intracluster Medium', ApJ, 453, 154-+.

Piner, B. G., Stone, J. M., Teuben, P. J., 1995, ‘Nuclear Rings and Mass Inflow in Hydrodynamic Simulations of Barred Galaxies', ApJ, 449, 508-+.

Pogge, R. W., Martini, P., 2002, 'Hubble Space Telescope Imaging of the Circumnuclear Environments of the CfA Seyfert Galaxies: Nuclear Spirals and Fueling', ApJ, 569, 624-640.

Rafanelli, P., Violato, M., Baruffolo, A., 1995, 'On the excess of physical companions among Seyfert galaxies', AJ, 109, 1546-1554.

Rees, M. J., 1984, 'Black Hole Models for Active Galactic Nuclei', ARA\&A, 22, 471-506.

Regan, M. W., Mulchaey, J. S., 1999, ‘Using HUBBLE SPACE TELESCOPE Imaging of Nuclear Dust Morphology to Rule Out Bars Fueling Seyfert Nuclei', AJ, 117, 2676-2694.

Risaliti, G., Elvis, M., 2004, 'A Panchromatic View of AGN', In: Barger, A. J. (Ed.), Supermassive Black Holes in the Distant Universe, Vol. 308 of Astrophysics and Space Science Library, pp. 187-+.

Rix, H.-W., White, S. D. M., 1992, 'Optimal estimates of line-of-sight velocity distributions from absorption line spectra of galaxies - Nuclear discs in elliptical galaxies', MNRAS, 254, 389-403.

Rogstad, D. H., Lockhart, I. A., Wright, M. C. H., 1974, 'Aperture-synthesis observations of H I in the galaxy M83.', ApJ, 193, 309-319.

Rousset, A., 1992, 'Contribution des méthodes numériques au dépouillement des données du spectrographe intégral de champ TIGER', PhD Thesis, Univ. J.Monnet de Saint-Etienne.

Rozas, M., Relaño, M., Zurita, A., Beckman, J. E., 2002, 'Fabry-Perot observations of the ionized gas in the spiral galaxy NGC 6951', A\&A, 386, 42-59.

Sakamoto, K., Okumura, S. K., Ishizuki, S., Scoville, N. Z., 1999, 'CO Images of the Central Regions of 20 Nearby Spiral Galaxies', ApJS, 124, 403-437.

Salviander, S., Shields, G. A., Gebhardt, K., Bonning, E. W., 2007, 'The Black Hole Mass-Galaxy Bulge Relationship for QSOs in the Sloan Digital Sky Survey Data Release 3', ApJ, 662, 131-144. 
Sancisi, R., Allen, R. J., Sullivan, III, W. T., 1979, 'Neutral hydrogen observations of the barred spiral galaxy NGC 5383', A\&A, 78, 217-225.

Sanders, D. B., Phinney, E. S., Neugebauer, G., Soifer, B. T., Matthews, K., 1989, 'Continuum energy distribution of quasars - Shapes and origins', ApJ, 347, $29-51$.

Saripalli, L., Mack, K.-H., 2007, 'A search for molecular gas in restarting radio galaxies', MNRAS, 376, 1385-1392.

Sarzi, M., Falcón-Barroso, J., Davies, R. L., Bacon, R., Bureau, M., Cappellari, M., de Zeeuw, P. T., Emsellem, E., Fathi, K., Krajnović, D., Kuntschner, H., McDermid, R. M., Peletier, R. F., 2006, 'The SAURON project - V. Integralfield emission-line kinematics of 48 elliptical and lenticular galaxies', MNRAS, 366, 1151-1200.

Sarzi, M., Rix, H.-W., Shields, J. C., Rudnick, G., McIntosh, D. H., Ho, L. C., Filippenko, A. V., Sargent, W. L. W., 2001, ‘Supermassive Black Holes from the Survey of Nearby Nuclei with STIS', In: Funes, J. G., Corsini, E. M. (Eds.), ASP Conf. Ser. 230: Galaxy Disks and Disk Galaxies, pp. 261-262.

Schinnerer, E., Eckart, A., Tacconi, L. J., 2000a, 'Distribution and Kinematics of the Circumnuclear Molecular Gas in the Seyfert 1 Galaxy NGC 3227', ApJ, $533,826-849$.

Schinnerer, E., Eckart, A., Tacconi, L. J., Genzel, R., 2000b, ‘Bars and warps traced by the molecular gas in the Seyfert 2 galaxy NGC 1068', ApJ, 533, 850-868.

Schmitt, H. R., 2001, 'The Frequency of Active and Quiescent Galaxies with Companions: Implications for the Feeding of the Nucleus', AJ, 122, 22432256.

Schmitt, H. R., Donley, J. L., Antonucci, R. R. J., Hutchings, J. B., Kinney, A. L., Pringle, J. E., 2003, 'A Hubble Space Telescope Survey of Extended [O III] $\lambda 5007 \AA$ Emission in a Far-Infrared-Selected Sample of Seyfert Galaxies: Results', ApJ, 597, 768-779.

Schödel, R., Ott, T., Genzel, R., Eckart, A., Mouawad, N., Alexander, T., 2003, 'Stellar Dynamics in the Central Arcsecond of Our Galaxy', ApJ, 596, 10151034.

Schoenmakers, R. H. M., Franx, M., de Zeeuw, P. T., 1997, 'Measuring nonaxisymmetry in spiral galaxies', MNRAS, 292, 349-+.

Seyfert, C. K., 1943, 'Nuclear Emission in Spiral Nebulae.', ApJ, 97, 28-+.

Shields, G. A., 1978, 'Thermal continuum from acretion disks in quasars', Nature, 272, 706-708. 
Shields, G. A., Gebhardt, K., Salviander, S., Wills, B. J., Xie, B., Brotherton, M. S., Yuan, J., Dietrich, M., 2003, 'The Black Hole-Bulge Relationship in Quasars', Vol. 583, pp. 124-133.

Shlosman, I., Begelman, M. C., Frank, J., 1990, 'The fuelling of active galactic nuclei', Nature, 345, 679-686.

Shlosman, I., Frank, J., Begelman, M. C., 1989, 'Bars within bars - A mechanism for fuelling active galactic nuclei', Nature, 338, 45-47.

Sicotte, V., Carignan, C., 1997, ‘NGC 5204: A Strongly Warped Magellanic Spiral.II.H I Kinematics and Mass Distribution', AJ, 113, 609-617.

Sikora, M., Stawarz, Ł., Lasota, J.-P., 2007, 'Radio Loudness of Active Galactic Nuclei: Observational Facts and Theoretical Implications', ApJ, 658, 815828.

Sil'chenko, O. K., Afanasiev, V. L., 2004, 'Inner Polar Rings in Regular Lenticular Galaxies', AJ, 127, 2641-2658.

Simões Lopes, R. D., Storchi-Bergmann, T., de Fátima Saraiva, M., Martini, P., 2007, 'A Strong Correlation between Circumnuclear Dust and Black Hole Accretion in Early-Type Galaxies', ApJ, 655, 718-734.

Sorrentino, G., Radovich, M., Rifatto, A., 2006, 'The environment of active galaxies in the SDSS-DR4', A\&A, 451, 809-816.

Statler, T., 1995, 'Bringing the cross-correlation method up to date', AJ, 109, 1371-1378.

Steidel, C. C., Sargent, W. L. W., 1991, 'Emission-line and continuum properties of 92 bright QSOs - Luminosity dependence and differences between radioselected and optically selected samples', ApJ, 382, 433-465.

Stocke, J. T., Morris, S. L., Weymann, R. J., Foltz, C. B., 1992, 'The radio properties of the broad-absorption-line QSOs', ApJ, 396, 487-503.

Storchi-Bergmann, T., 1991, 'On the ratio (N II)/H-alpha in the nucleus of Seyfert 2 and LINER galaxies', MNRAS, 249, 404-408.

Terlevich, R., Tenorio-Tagle, G., Franco, J., Melnick, J., 1992, 'The starburst model for active galactic nuclei - The broad-line region as supernova remnants evolving in a high-density medium', MNRAS, 255, 713-728.

Thean, A. H. C., Mundell, C. G., Pedlar, A., Nicholson, R. A., 1997, 'A neutral hydrogen study of the Seyfert galaxy NGC 5033', MNRAS, 290, 15-24.

Thornley, M. D., Mundy, L. G., 1997, ‘Are Flocculent Spirals Devoid of Density Waves? Gas Morphology and Kinematics in NGC 5055’, ApJ, 484, 202-+. 
Tremaine, S., Gebhardt, K., Bender, R., Bower, G., Dressler, A., Faber, S. M., Filippenko, A. V., Green, R., Grillmair, C., Ho, L. C., Kormendy, J., Lauer, T. R., Magorrian, J., Pinkney, J., Richstone, D., 2002, 'The Slope of the Black Hole Mass versus Velocity Dispersion Correlation', ApJ, 574, 740-753.

Treu, T., Malkan, M. A., Blandford, R. D., 2004, 'The Relation Between Black Hole Mass and Velocity Dispersion at z 0.37', ApJ, 615, L97-L100.

Tully, R. B., 1974, 'The Kinematics and Dynamics of M51. 11. Axisymmetric Properties', ApJS, 27, 437-+.

Ulrich, M.-H., 2000, 'The active galaxy NGC 4151: Archetype or exception?', AEA Rev., 10, 135-178.

Ulvestad, J. S., Ho, L. C., 2001, 'Statistical Properties of Radio Emission from the Palomar Seyfert Galaxies', ApJ, 558, 561-577.

Urry, C. M., Padovani, P., 1995, ‘Unified Schemes for Radio-Loud Active Galactic Nuclei', PASP, 107, 803-+.

van Albada, G. D., 1980, ‘New high resolution radio observations of NGC 4258. II - NGC 4258 as a spiral galaxy', A\&A, 90, 123-133.

van der Kruit, P. C., Oort, J. H., Mathewson, D. S., 1972, 'The Radio Emission of NGC 4258 and the Possible Origin of Spiral Structure', A\&A, 21, 169-+.

van der Marel, R. P., Franx, M., 1993, 'A new method for the identification of non-Gaussian line profiles in elliptical galaxies', ApJ, 407, 525-539.

Vazdekis, A., 1999, 'Evolutionary Stellar Population Synthesis at $2 \AA$ Spectral Resolution', ApJ, 513, 224-241.

Vila-Vilaro, B., Robinson, A., Perez, E., Axon, D. J., Baum, S. A., GonzalezDelgado, R. M., Pedlar, A., Perez-Fournon, I., Perry, J. J., Tadhunter, C. N., 1995, 'Circum-nuclear gas flows in NGC 4151.', A\&A, 302, 58-+.

Vollmer, B., 2003, ‘NGC 4654: Gravitational interaction or ram pressure stripping?', A\&A, 398, 525-539.

Vollmer, B., Cayatte, V., Boselli, A., Balkowski, C., Duschl, W. J., 1999, ‘Kinematics of the anemic cluster galaxy NGC 4548. Is stripping still active?', A\&A, 349, 411-423.

Wada, K., 2004, 'Fueling Gas to the Central Region of Galaxies', In: Ho, L. C. (Ed.), Coevolution of Black Holes and Galaxies, pp. 186-+.

Whitmore, B. C., Forbes, D. A., Rubin, V. C., 1988, 'Rotation curves for spiral galaxies in clusters. II - Variations as a function of cluster position', ApJ, 333, 542-560.

Wiita, P. J., 2006, 'Active Galactic Nuclei: Unification, Blazar Variability and the Radio Galaxy / Cosmology Interface', ArXiv Astrophysics e-prints. 
Wilson, A. S., Colbert, E. J. M., 1995, 'The difference between radio-loud and radio-quiet active galaxies', ApJ, 438, 62-71.

Wong, T., Blitz, L., Bosma, A., 2004, 'A Search for Kinematic Evidence of Radial Gas Flows in Spiral Galaxies’, ApJ, 605, 183-204.

Wozniak, H., Champavert, N., 2006, 'Lifetime of nuclear velocity dispersion drops in barred galaxies', MNRAS, 369, 853-859.

Wozniak, H., Combes, F., Emsellem, E., Friedli, D., 2003, ‘Numerical simulations of central stellar velocity dispersion drops in disc galaxies', A\&A, 409, 469-477.

Yates, M. G., Miller, L., Peacock, J. A., 1989, 'The cluster environments of powerful, high-redshift radio galaxies', MNRAS, 240, 129-166.

Zhang, X., Wright, M., Alexander, P., 1993, 'High-Resolution CO and $\mathrm{H}$ i Observations of the Interacting Galaxy NGC 3627', ApJ, 418, 100-+. 


\begin{abstract}
This thesis work investigates two main points on the researches on active galactic nuclei : the mechanisms which transport gas and the role of the host galaxy on nuclear activity. We conducted an observational comprehensive and statistical study of the gas and stars on a sample of active and inactive galaxies, on different spatial scales. We obtained then radio and optical spectroscopic data, using the Very Large Array (VLA) and the Integral Field Spectrograph (IFS) SAURON. Our results show that the stellar kinematics is regular while the gas is perturbed, in the central regions of active galaxies. Those perturbations suggest a link between the dynamics in centre of galaxies and the mechanisms which fuel the active nuclei. Finally, we combine the radio and optical data and analyse the kinematics from the outskirts to the central regions of the galaxies. Such study allows investigating the perturbations related to the feeding of the nuclear activity at different spatial scales.
\end{abstract}

\title{
Résumé
}

Ce travail de thèse s'articule autour de deux questions scientifiques importantes à propos des galaxies actives : quels sont les mécanismes transportant le gaz et quel est le rôle de la galaxie sur l'activité nucléaire? Nous avons donc mené une étude observationnelle approfondie et statistique du gaz et des étoiles, pour comparer la morphologie et cinématique des galaxies actives et non-actives sur différentes échelles spatiales, en utilisant des données spectroscopiques optique et radio. Nos résultats montrent que dans les régions centrales des galaxies actives la cinématique des étoiles est régulière alors que le gaz est perturbé. Ces perturbations suggèrent un lien entre la dynamique au centre des galaxies et les mécanismes d'alimentation du noyau actif. Enfin les données radio et optique sont combinées pour analyser la cinématique galactique dans son ensemble. Cette étude nous permet de sonder à différentes échelles spatiales les perturbations liées à l'alimentation du noyau actif. 